\title{
TWO-DIMENSIONAL SPATIAL IMAGING OF CHARGE TRANSPORT IN GERMANIUM CRYSTALS AT CRYOGENIC TEMPERATURES
}

\author{
A DISSERTATION \\ SUBMITTED TO THE DEPARTMENT OF PHYSICS \\ AND THE COMMITTEE ON GRADUATE STUDIES \\ OF STANFORD UNIVERSITY \\ IN PARTIAL FULFILLMENT OF THE REQUIREMENTS \\ FOR THE DEGREE OF \\ DOCTOR OF PHILOSOPHY
}

Robert A. Moffatt

March 2016

Operated by Fermi Research Alliance, LLC under Contract No. DE-AC02-07CH11359 with the United States Department of Energy 
(C) 2016 by Robert Alexander Moffatt. All Rights Reserved.

Re-distributed by Stanford University under license with the author.

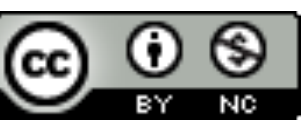

This work is licensed under a Creative Commons AttributionNoncommercial 3.0 United States License.

http://creativecommons.org/licenses/by-nc/3.0/us/

This dissertation is online at: http://purl.stanford.edu/dm346nh6357 
I certify that I have read this dissertation and that, in my opinion, it is fully adequate in scope and quality as a dissertation for the degree of Doctor of Philosophy.

Blas Cabrera, Primary Adviser

I certify that I have read this dissertation and that, in my opinion, it is fully adequate in scope and quality as a dissertation for the degree of Doctor of Philosophy.

Peter Graham

I certify that I have read this dissertation and that, in my opinion, it is fully adequate in scope and quality as a dissertation for the degree of Doctor of Philosophy.

Kent Irwin

Approved for the Stanford University Committee on Graduate Studies.

Patricia J. Gumport, Vice Provost for Graduate Education

This signature page was generated electronically upon submission of this dissertation in electronic format. An original signed hard copy of the signature page is on file in University Archives. 
(C) Copyright by Robert A. Moffatt 2016

All Rights Reserved 
I certify that I have read this dissertation and that, in my opinion, it is fully adequate in scope and quality as a dissertation for the degree of Doctor of Philosophy.

\section{(Blas Cabrera) Principal Adviser}

I certify that I have read this dissertation and that, in my opinion, it is fully adequate in scope and quality as a dissertation for the degree of Doctor of Philosophy.

(Kent Irwin)

I certify that I have read this dissertation and that, in my opinion, it is fully adequate in scope and quality as a dissertation for the degree of Doctor of Philosophy.

(Peter Graham)

Approved for the Stanford University Committee on Graduate Studies 


\section{Preface}

In this dissertation, I describe a novel apparatus for studying the transport of charge in semiconductors at cryogenic temperatures. The motivation to conduct this experiment originated from an asymmetry observed between the behavior of electrons and holes in the germanium detector crystals used by the Cryogenic Dark Matter Search (CDMS).

This asymmetry is a consequence of the anisotropic propagation of electrons in germanium at cryogenic temperatures. To better model our detectors, we incorporated this effect into our Monte Carlo simulations of charge transport. The purpose of the experiment described in this dissertation is to test those models in detail.

Our measurements have allowed us to discover a shortcoming in our most recent Monte Carlo simulations of electrons in germanium. This discovery would not have been possible without the measurement of the full, two-dimensional charge distribution, which our experimental apparatus has allowed for the first time at cryogenic temperatures. 


\section{Acknowledgements}

The work described in this dissertation could not have happened without the many contributions of my friends, colleagues, and mentors. I would especially like to express my appreciation to my research advisor, Prof. Blas Cabrera, for his guidance and mentorship over the last seven years. I first met Blas back when I was still unsure of which research topic I wished to pursue. Blas offered me a one-quarter rotation with his group in order to work on a calculation which had caught my interest. ${ }^{1}$ As I learned more about the Cryogenic Dark Matter Search, I was excited by the prospect of joining a research collaboration which aimed to tackle one of the great unsolved problems in physics. However, what really motivated me to join Blas' group was not the research topic or the experiment, but the time and attention which Blas gave to me and my work.

I am glad that I was given the opportunity to join Blas' group, and I have enjoyed working with him immensely. I have learned a great deal about condensed matter physics from him, and I have always been amazed at his intuitive understanding of the behavior of materials and processes in conditions which are so far removed from everyday experience. It was once jokingly said of William Shockley that his intuition regarding semiconductors came from an ability to see electrons. I wouldn't be surprised to learn that Blas has the ability to see phonons, quasiparticles, and Cooper pairs.

Blas is also an excellent experimentalist, and his advice and guidance have been invaluable, especially while troubleshooting. I remember one day in particular ${ }^{2}$, April

\footnotetext{
${ }^{1}$ This is the work which is reproduced in Appendix F.

${ }^{2}$ I remember the date because of the somewhat exasperated summary I recorded in my experimental notes of everything that had failed to work properly.
} 
30, 2013, when I first tested the charge transport system in the ${ }^{3} \mathrm{He}$ cryostat at a temperature of $4 \mathrm{~K}$. Absolutely nothing was working. I carefully documented the behavior of the system, but I had no idea how to proceed. I was quite disheartened when I described the state of affairs to Blas, but he very quickly devised an experimentum crucis which allowed us to disentangle a multitude of simultaneous problems. Since then, I have tried to follow Blas' example and to emulate his techniques in my experimental work.

I also fondly remember the many late nights I spent with Blas and his students up at the Stanford Student Observatory. Blas is an avid amateur astronomer, and was the instructor for one of the introductory astronomy courses at Stanford. I volunteered to be his teaching assistant for the course, even though I had no prior experience in astronomy. Both I and the students learned a great deal from Blas, and his enthusiasm was infectious. Since then, I have taken up amateur astronomy as a hobby myself, and used my telescope privileges at the Student Observatory to share this experience with my friends and family.

Most importantly, I have found that Blas exhibits a dedication to his students and a concern for their development as physicists and as individuals which is both uncommon and exemplary. I am lucky to have had the opportunity to be mentored by him, especially since I joined his group as one of his last three graduate students.

Blas has had a personal impact, not just on me, but broadly throughout the field of physics - a fact which I learned at a banquet that was held in 2012 to recognize Blas' achievements and celebrate his career. At the banquet, many of Blas' former students, now professors, praised not only his meticulous attention to detail and his deep physical intuition, but also the interest and devotion he showed to the work of his students, the personal care he took in their growth and development as a physicists and problem-solvers, and the advice and support he provided to them in pursuing their future careers.

As Blas' last official graduate student, I therefore feel the duty to thank him, not only on behalf of myself, but also on behalf of all of the students who have benefited from his mentorship and support over the years. Blas' excellence as a teacher and mentor is evidenced not only by the debts of gratitude expressed by all 
of his students who have gone before me, but also in the many successful careers of his former protégés.

One of Blas' former students for whose mentorship I am especially grateful is Prof. Betty Young. She splits her time between teaching at Santa Clara University, and doing research as part of our group at Stanford, and her drive and dedication are truly inspiring. Despite her busy schedule, she still takes time to help me and my fellow students in the lab, offer feedback and corrections on papers and posters, and provide valuable advice and guidance in our future career plans. Her cheerful demeanor and incredible work ethic have always been both motivational and uplifting.

I would also like to recognize and thank all of my friends and collaborators who have worked with me on the charge transport experiment and contributed to its success. Both Peter Redl and Kyle Sundqvist are responsible for the charge transport simulations and theory. Peter, in particular, spent many hours incorporating new physical effects into the Monte Carlo code to test new theories motivated by the experimental data. He has recently started a new job at Google, which I told him is the perfect fit for someone who once tied up a majority of the computing resources at SLAC while running our Monte Carlo code.

I am also thankful to our SLAC group, including Matt Cherry, Astrid Tomada, John Mark Kreikebaum, Noah Kurinsky, Paul Brink, and Richard Partridge, who work on the fabrication of our detectors, and who made the test crystals which were used in this experiment. Matt Cherry, in particular, spent considerable time wirebonding our crystal samples and fixing small surface defects under the microscope with his experienced and steady hands. John Mark showed me how to use the X-ray diffraction machine and performed the X-ray diffraction measurements which revealed a slight offset in our crystal axes.

I would also like to recognize our collaborators at U.C. Berkeley. Arran Phipps has done substantial research into the mobility and trapping of charge carriers in high purity germanium. By combining his results with those described in this dissertation, we have been able to characterize both the spatial and temporal properties of charge transport for a better comparison between experiment and theory. Prof. Bernard Sadoulet has also provided insight and guidance in both the interpretation of our 
experimental results, and in the design of new measurements to test our theories. Kyle Sundqvist, who completed his doctoral work on the theory of charge transport at U.C. Berkeley under Prof. Sadoulet, is now a professor of Electrical Engineering at Texas A\&M University.

Three undergraduate students also deserve recognition for the time and effort they spent on this experiment. Fedja Kadribasic worked with our group for several years during the early phase of this experiment. His measurements of our piezoelectric actuator were useful in characterizing the feasibility of that system, and were the basis of our decision to switch from piezoelectrics to MEMS technology. He and I also spent many hours in the Varian machine shop making the copper pieces of our scanning apparatus and characterizing the behavior of the system in the ${ }^{3} \mathrm{He}$ cryostat. He has since graduated from Stanford, and is now in the Ph.D. program at Texas A\&M University.

Brendan Corcoran helped to run the ${ }^{3}$ He cryostat during the summer of 2014 when most of the experimental results reported in this dissertation were taken. His work in characterizing the thermal properties of the cryostat, described in $\S 6.5$, was very useful for understanding the response of the system to heat sources, and for analyzing the feasibility of active temperature control.

James Allen helped to run the ${ }^{3} \mathrm{He}$ cryostat during the summer of 2015 as part of our ongoing effort to measure the charge transport properties of silicon. He also designed and tested an improved copper heat strap for our $4 \mathrm{~K}$ amplifier circuit, and measured the equilibrium temperature of our ${ }^{3} \mathrm{He}$ still as a function of power applied to the charcoal sorb. Based on his measurements, plotted in Figure G.25, he wrote a PID control loop which was capable of maintaining the payload at a constant temperature without decreasing the hold time, which will be very useful for future measurements of charge transport at different temperatures.

I am also grateful to my mentors and colleagues who taught me how to operate the ${ }^{3} \mathrm{He}$ cryostat and helped me to run it. Jeff Yen and Betty Young both made themselves available on many occasions to help with helium transfers, often at odd and inconvenient hours. Alex Contryman and Dominik Rastawicki helped keep the experiment cold by lending us some of their liquid helium whenever we found ourselves 
in a pinch. Ben Shank, who performed his doctoral research using the same cryostat, taught me how to use it and helped me to run it for many months before he graduated.

Our ${ }^{3} \mathrm{He}$ cryostat was loaned to us by Prof. Sarah Church's research group, which previously used it as a CMB telescope. Keith Thompson was one of the original researchers who used this cryostat. Even though he is not a member of our research group, he has been very generous in sharing his knowledge and experience with me, and he has provided vital help in diagnosing the sources of problems on multiple occasions.

I would also like to express my appreciation to the machinists of the Varian machine shop, especially Karlheinz Merkle and Mehmet Solyali. Not only did they make many of the complex parts of our scanning apparatus, Mehmet always made himself available to provide tools and guidance for my machining tasks, and to teach me new skills.

The engineers and CEO of the company, Mirrorcle, also deserve recognition for the many hours they spent on the phone with us discussing the implementation of their MEMS mirror into our system. When their standard chip failed at temperatures below $4 \mathrm{~K}$, they worked with us to develop a modified version that worked down to a temperature of $0.4 \mathrm{~K}$, which was a key component in the success of our experiment.

I have greatly enjoyed the time I have spent working at Stanford as part of the Cryogenic Dark Matter Search collaboration - a fact which is in large part due to the many wonderful friends I have met since coming to Stanford. Now that Ben Shank has graduated, I will miss the many gracious dinner invitations which he and his wonderful wife, Katie, extended to me, Jeff Yen, Kristi Schneck, Peter Redl, Daniel Nagasawa, and Fedja Kadribasic, while we were all working together in the Cabrera Lab. Ben has since moved with his wife and son to Michigan to be near family, and he currently works at Thermotron, where he serves as their in-house physics expert.

I am also glad to have met and worked with my friend and colleague, Jeff Yen, who studies quasiparticle transport in superconducting aluminum in order to improve the phonon sensors on our detector crystals. In addition to his scientific skill, Jeff has a keen business sense, and is always alert to potential opportunities. He possesses an infectious excitement and imperturbable optimism which attracts many friends. I am 
happy to have met many new people through Jeff, including my good friend, Long Sun.

The Cryogenic Dark Matter Search collaboration has been a wonderful group to work with. I am grateful to Matt Pyle for his technical discussions and valuable insights, to Steve Yellin for his computer expertise, to Joel Sander for teaching me how to use the DAQ at our experimental facility in Soudan, Minnesota, and to Dan Bauer for always being just a phone call away whenever something wasn't working at Soudan. I also appreciate all of our mine crew at Soudan, including Dan DeVaney, Jeff Gunderson, Denise Osterholm, Dave Saranen, Jerry Meier, and Jim Beaty, who always made me feel welcome whenever I was on shift, and who were always available to help fix any problems that arose in the mine, or to go out for wings at the Good Ol' Days when our work for the day was done.

I would also like to express my appreciation for the administrative staff of the Physics Department, especially Marcia Keating, who does an incredible job as the administrator for multiple research groups in Varian. She has always gone above and beyond her duties, including taking excellent care of my plants when I was away on trips. Elizabeth Palmquist and Scott Barton have been very helpful with property transfers between labs, and they are always interested to hear how my research is going. I also wish to express my gratitude to everyone in the Varian Main Office, including Violet Catindig, Elva Carbajal, and Maria Frank. I am thankful to Maria, in particular, for helping to answer all of my questions on topics ranging from course waivers to graduation requirements. She always seems to have a solution to every problem.

In addition to the connections I have made through my physics research, I am also grateful for the friends and mentors whom I have met through the Stanford Amateur Radio Club, which is federally licensed under the call sign W6YX. Sawson Taheri was the president of W6YX when I first joined the club, and he has since become a steadfast and loyal friend. I value his humor, his intuition for electronics, and his unique perspective on life, all three of which he shares generously.

I am also indebted to Professors David Leeson and John Pauly, who provided me with mentorship and support during the past year when I served as the president of 
W6YX. From recruiting new members, to making club decisions, they were always available to provide help and guidance.

I also wish to express my gratitude for the Robert and Marvel Kirby Fellowship, which was established by Robert Kirby, a Stanford alumnus, and his wife, Marvel Kirby, for the purpose of supporting graduate research at Stanford. By providing financial support for my first three years of studies, this fellowship gave me the freedom to explore research opportunities without financial constraint, and was especially beneficial during the the early stage of my graduate career when I had yet to decide my future path.

Of course, the fact that I have been given the opportunity to pursue an academic career is something which I owe to both my parents and my grandfather who fully covered all of my undergraduate tuition costs. They, and my entire family, have been nothing but supportive of all my academic goals.

My brother, Ryan, is deserving of special thanks. Teaching has been a passion of mine from an early age, and I wish to thank Ryan for allowing me to be his teacher from time to time when we were growing up. I appreciate his patience whenever he needed my tutoring in physics or mathematics, especially since I was usually much more excited to discuss his schoolwork than he was, and I loved to go off on digressions. ${ }^{3}$

Finally, and most importantly, I wish to thank my parents for all of the love and encouragement that they have given me and my brother for all of our lives. My love of science and my love of learning are directly attributable to my parents, who realized that we will never cease to learn so long as we find joy in the act of learning. They always found ways to provide me with opportunities to seek my highest potential, even at an early age, whether it was by buying me an electronics kit when I was in 3rd grade, by taking me to the U.S. Patent Office to do a patent search when I was still in elementary school, by driving over eight hours back and forth every day to take me to an inventor's camp for an entire summer, or by allowing me to keep a desk

\footnotetext{
${ }^{3}$ However, if you ask him he will tell you that was nothing in comparison to the patience he showed most weekends when he and my mother waited for me, often for hours, as I rummaged through the bins at our local RadioShack looking for parts for my next electronics project. He admitted to feeling a certain relief when he heard that RadioShack has recently gone out of business.
} 
filled with electronics projects in constant disarray.

It is easy to take what one is given for granted, especially as a child, and it is therefore only as I have grown older, and learned more about the world, that I have come to appreciate how truly remarkable and special my parents have always been. They have shown, and continue to show, a level of love, devotion, and self-sacrifice for their children which, in my experience, is unsurpassed. I am reminded of the legend of Cornelia Africana, the daughter of Scipio, who was once asked why she wore no jewelry. In response, she pointed to her two sons, explaining, "these are my jewels." I cannot count the number of times I have heard my parents say these very same words about my brother and me.

I have always admired my parents' wisdom and maturity, and I always seek to make them proud. I cannot think of any two people who have contributed more to my education, or feel more pride in what I have achieved. It is therefore with feelings of both deepest love and sincerest gratitude that I dedicate this dissertation to them. 


\section{Contents}

Preface $\quad$ iv

Acknowledgements $\quad$ V

1 Introduction 1

2 Dark Matter and CDMS 3

2.1 Indirect Evidence for Dark Matter . . . . . . . . . . . . . . . 3

2.2 Dark Matter Candidates . . . . . . . . . . . . . . . . 7

2.3 Direct Detection of Dark Matter . . . . . . . . . . . . . 8

3 CDMS Detector Physics 9

3.1 Particle Interactions . . . . . . . . . . . . . . . . . . 9

3.2 Energy Collection . . . . . . . . . . . . . . . . 13

3.3 Shielding and Discrimination Power . . . . . . . . . . . . 16

4 Anomalous Effects in CDMS Crystals 19

4.1 Charge Sharing Asymmetry . . . . . . . . . . . . . . 19

4.2 Shorted Charge Channel . . . . . . . . . . . . . . 24

4.3 Importance of Accurate Modeling . . . . . . . . . . . . . . . . 24

5 Charge Transport in Semiconductors $\quad 26$

5.1 Band Structure and Bloch Wavefunctions . . . . . . . . . . . . 26

5.2 Indirect-Gap Semiconductors . . . . . . . . . . . . . . 28

5.3 Semiclassical Model of Charge Transport . . . . . . . . . . . . . . . . 29 
5.4 Scattering Processes . . . . . . . . . . . . . . . . . 32

5.5 Electron-Phonon Interaction . . . . . . . . . . . . . . . . . . 34

5.6 Carrier Mobility . . . . . . . . . . . . . . . 36

5.7 Electron Anisotropy . . . . . . . . . . . . . . . . . . . 39

6 Experimental Setup $\quad 43$

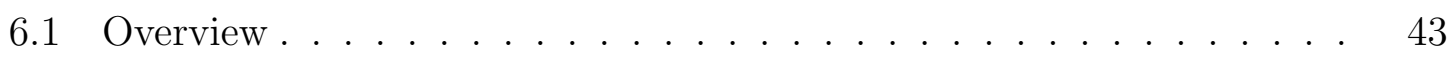

6.2 Crystal Sample . . . . . . . . . . . . . . . . . 43

6.3 Optical System . . . . . . . . . . . . . . . . . 44

6.4 Amplifier Circuit . . . . . . . . . . . . . . . . 48

$6.5{ }^{3}$ He Cryostat . . . . . . . . . . . . . . . . . 53

6.6 Control System . . . . . . . . . . . . . . . . 56

7 Experimental Methods $\quad 58$

7.1 Neutralization . . . . . . . . . . . . . . . . . . 58

7.2 Scanning Process . . . . . . . . . . . . . . . . . . 58

7.3 Maximum-Likelihood Fits . . . . . . . . . . . . . . . . 59

7.4 Pulse Gain . . . . . . . . . . . . . . . . . . . . . . . . 60

7.5 Charge Collection Estimates . . . . . . . . . . . . . . 62

7.6 Charge Noise . . . . . . . . . . . . . . . . 63

7.7 Raster Scan Patterns and Hysteresis . . . . . . . . . . . . . 65

7.8 Edge Effects . . . . . . . . . . . . . . . . . . . 67

7.9 Position Calibration . . . . . . . . . . . . . . 67

7.10 Inter-Valley Scattering Length . . . . . . . . . . . . . 69

8 Experimental Results $\quad 70$

8.1 Two-Dimensional Charge Density . . . . . . . . . . . . . 70

8.2 Inter-Valley Scattering Length . . . . . . . . . . . . . . 73

8.3 Leakage Current and Charge Trapping . . . . . . . . . . . . . . 74

$\begin{array}{lll}9 & \text { Discussion } & 75\end{array}$

10 Conclusions and Future Work $\quad 79$ 
A An Isothermal Cloud $\quad 81$

A.1 Newton's Laws and the Equation of State . . . . . . . . . . . . . 81

A.2 A Differential Equation for the Density Profile . . . . . . . . . . 83

A.3 A Simple Solution . . . . . . . . . . . . . . . . . 83

A.4 A Solution with Realistic Boundary Conditions . . . . . . . . . . 84

A.5 Comparison to Observation . . . . . . . . . . . . . 87

B WIMP Scattering Cross-Section $\quad 89$

B.1 Kinematics ......................... 89

B.2 Scattering Amplitude . . . . . . . . . . . . . . . . 90

B.3 Scattering Cross-Section . . . . . . . . . . . . . . . . 92

B.4 Composite Particles . . . . . . . . . . . . . . . . 93

B.5 Bound Particles . . . . . . . . . . . . . . . . . . 93

C Quasiballistic Diffusion of Phonons $\quad 94$

C.1 The Ballistic Transport of Particles . . . . . . . . . . . . . . . . . 94

C.2 Elastic Scattering . . . . . . . . . . . . . . . . 96

C.3 Time Evolution . . . . . . . . . . . . . . . . . . . 97

C.4 The Diffusion Equation . . . . . . . . . . . . . . . . . 98

$\begin{array}{ll}\text { D Diffusion in a Lossy Medium } & 100\end{array}$

D.1 The Diffusion Equation . . . . . . . . . . . . . . . . . 100

D.2 The Integrated Absorption . . . . . . . . . . . . . . . . . . 101

D.3 A Point Source in the Presence of Perfect Absorbers . . . . . . . . . . 103

D.4 A Point Source in One Dimension . . . . . . . . . . . . . . 105

D.5 Mean Collection Fraction in One Dimension . . . . . . . . . . . . 107

D.6 Mean Collection Fraction in an Annulus . . . . . . . . . . . . . . . 108

D.7 Comparison of 1D and 2D Solutions . . . . . . . . . . . . 111

E Linear TES Response 112

E.1 The Electro-Thermal Feedback of a Transition-Edge Sensor . . . . . . 112

E.2 The Resistance vs. Temperature . . . . . . . . . . . . . . . . . 113 
E.3 The Dynamics of a Transition-Edge Sensor . . . . . . . . . . . . . . 115

E.4 The Loss of Deposited Energy to the Substrate . . . . . . . . . . . . 116

E.5 The Integrated Electrical Power . . . . . . . . . . . . . . . . . . 117

$\begin{array}{lll}\text { F Electron-Phonon Interactions } & 119\end{array}$

F.1 Generalized Vectors . . . . . . . . . . . . . . . . . . . . 119

F.2 Euler-Lagrange Equations . . . . . . . . . . . . . . . . 120

F.3 Linear Operators . . . . . . . . . . . . . . . . . . . . . . . . 121

F.4 The Schrödinger Lagrangian . . . . . . . . . . . . . . . . . . . . 122

F.5 The Elastic Lagrangian . . . . . . . . . . . . . . . . . . . . . . 124

F.6 The Electron-Phonon Interaction . . . . . . . . . . . . . . . 127

F.7 The Continuum Model . . . . . . . . . . . . . . . . . . 129

F.8 Transformation to Fourier Space . . . . . . . . . . . . . . . . . 130

F.9 Canonical Quantization . . . . . . . . . . . . . . . 133

F.10 The Interaction Hamiltonian . . . . . . . . . . . . . . . . . . . 134

F.11 Phonon Emission and Absorption Rates . . . . . . . . . . . . . 135

F.12 Differential Intra-Valley Scattering Rates . . . . . . . . . . . . . . . 138

F.13 Isotropic Intra-Valley Scattering Rates . . . . . . . . . . . . . . . 140

F.14 The High Temperature Limit . . . . . . . . . . . . . . . . . . 142

F.15 The Low Temperature Limit . . . . . . . . . . . . . . . . . . 143

G Experimental Setup - Supplemental Material 144

G.1 Mechanical Drawings . . . . . . . . . . . . . . . . . . 144

G.2 Amplifier Circuit . . . . . . . . . . . . . . . . 154

G.3 Photos .............................. 155

G.4 Transfer Function . . . . . . . . . . . . . . . 163

G.5 Noise . . . . . . . . . . . . . . . . . 165

G.6 ${ }^{3} \mathrm{He}$ Cryostat . . . . . . . . . . . . . . . 166

G.7 Control System . . . . . . . . . . . . . . . . . . . . . . . . . 168

H Maximum Likelihood Linear Fits $\quad 169$

H.1 Signal Vectors and Inner Products . . . . . . . . . . . . . . . . 169 
H.2 Gaussian Noise . . . . . . . . . . . . . . . . . . . . . . . 171

H.3 Linear Curve Fitting . . . . . . . . . . . . . . . . . . . . . . . 172

H.4 Signal to Noise Ratio . . . . . . . . . . . . . . . . . . . . . . . 174

H.5 Fits with Only One Parameter . . . . . . . . . . . . 176

I Piezoelectric Resonators $\quad 177$

I.1 The Elastic Lagrangian of the Piezo Actuator . . . . . . . . . . . . . 177

I.2 Electromagnetic Lagrangian and Coupling . . . . . . . . . . . . . . . 180

I.3 The Lagrangian and the Euler-Lagrange Equations . . . . . . . . . . 182

I.4 Induced Charge . . . . . . . . . . . . . . . . . . . 183

I.5 Dissipation and Impedance . . . . . . . . . . . . . . . . . . . . . . 184

I.6 Piezo Properties and Electrically Measurable Quantities . . . . . . . . 186

$\begin{array}{llr}\text { J Matlab Code } & 188\end{array}$

$\begin{array}{ll}\text { Bibliography } & 222\end{array}$ 


\section{List of Tables}

5.1 Hole and electron masses in $\mathrm{Si}$ and Ge. . . . . . . . . . . . . . . . . 31

5.2 Parameters for Phonon Emission and Absorption. . . . . . . . . . 36

G.1 Poles and zeros for amplifier transfer functions. . . . . . . . . . 163

I.1 Piezoelectric Material Properties . . . . . . . . . . . . . . . . 187

I.2 Resonator Properties . . . . . . . . . . . . . . . . . . . . 187 


\section{List of Figures}

2.1 Astrophysical evidence for dark matter. . . . . . . . . . . . . . 4

2.2 N-body simulation of a dark matter halo. . . . . . . . . . . . . 5

2.3 Cosmological evidence for dark matter. . . . . . . . . . . . . 6

$2.4 \Lambda$ CDM model parameters from Planck. . . . . . . . . . . . . . 6

2.5 Dark matter candidates from particle physics. . . . . . . . . 7

2.6 Non-gravitational searches for dark matter. . . . . . . . . . . . . 8

3.1 Dark Matter Interaction with Ge. . . . . . . . . . . . . . 10

3.2 Current limits on WIMP interaction cross-section. . . . . . . . . . 11

3.3 Photograph of CDMS iZIP detector. Diagram of surface pattern. . . 14

3.4 Response of CDMS iZIP to localized energy deposition. . . . . . . . 15

3.5 Soudan Mine. . . . . . . . . . . . . . . . . . . . . 16

3.6 CDMS Experimental Apparatus. . . . . . . . . . . . . . 17

3.7 CDMS detector background discrimination. . . . . . . . . 18

4.1 Haller dislocation-free crystal. . . . . . . . . . . . . . . 20

4.2 Asymmetric charge sharing in Haller crystal. . . . . . . . . . . . 21

4.3 Charge sharing in iZIP detectors. . . . . . . . . . . . . 22

4.4 Monte Carlo simulation of electron and hole propagation. . . . . . . 23

4.5 Monte Carlo simulation of CDMS detector with shorted channel. . . . 25

5.1 Valance and conduction bands in Ge. . . . . . . . . . . 27

5.2 Three-dimensional plots of Si and Ge conduction bands. . . . . . . . 29

5.3 Electron scattering processes in Ge. . . . . . . . . . . . . . 34 
5.4 Kinematics of Phonon Emission. . . . . . . . . . . . . . . . . 37

5.5 Carrier drift velocity. . . . . . . . . . . . . . . . . . 38

5.6 3D Monte Carlo simulation of electron propagation in Ge. . . . . . . 42

6.1 Microscope images of surfaces of Ge crystal sample. . . . . . . . . . . 44

6.2 Annotated photograph of experimental payload. . . . . . . . . . . 45

6.3 Diagram and photograph of laser scanning system. . . . . . . . . . . 46

6.4 Photographs of MEMS mirrors. . . . . . . . . . . . . . . . . . 47

6.5 Measurement laser spot focus. . . . . . . . . . . . . . . . . . . 47

6.6 Simplified schematic of the amplifier circuit. . . . . . . . . . . . . . 49

6.7 Transfer functions for both channels of the amplifier circuit. . . . . . 51

6.8 Amplifier noise spectra. . . . . . . . . . . . . . . . . . . . 52

6.9 High-bandwidth impulse response curves. . . . . . . . . . . . . . . . 53

6.10 Diagram of ${ }^{3} \mathrm{He} /{ }^{4} \mathrm{He}$ cooling system. . . . . . . . . . . . . . . . . . . 54

6.11 Load line of ${ }^{3} \mathrm{He}$ fridge. . . . . . . . . . . . . . . . . . 55

6.12 Photograph of the experimental payload mounted in the ${ }^{3}$ He cryostat. $\quad 56$

6.13 Simplified block diagram of the control system. . . . . . . . . . . . 57

7.1 Raw Data from Crystal Scan. . . . . . . . . . . . . . . . . . . 60

7.2 Low-bandwidth pulse train templates. . . . . . . . . . . . . . . . . 61

7.3 Low-bandwidth charge pulses with least-squares fits. . . . . . . . . . 62

7.4 Horizontal and vertical raster scans. . . . . . . . . . . . . . . 66

7.5 Image Processing. . . . . . . . . . . . . . . . . . . . . . . 67

7.6 Position calibration. . . . . . . . . . . . . . . . 68

7.7 Regions defining IV Scattering. . . . . . . . . . . . . . . . . 69

8.1 Hole charge density patterns at 600mK. . . . . . . . . . . . . 71

8.2 Electron charge density patterns at 600mK. . . . . . . . . . . 72

8.3 Electron inter-valley scattering length vs. electric field at $600 \mathrm{mK}$. . . 73

8.4 Charge collection and crystal conduction vs. electric field. . . . . . . 74

9.1 Detailed comparison between data and simulation. . . . . . . . . . 76

9.2 Comparison of Redl and Sundqvist simulations. . . . . . . . . . . . . 77 
9.3 Lateral spreading of electron clusters. . . . . . . . . . . . . . . . 78

A.1 Dark matter halo density profile. . . . . . . . . . . . . . . 85

A.2 Dark matter halo cumulative mass. . . . . . . . . . . . . . . . 86

A.3 Galactic rotation curve model. . . . . . . . . . . . . . . . 87

A.4 Galactic rotation curves. . . . . . . . . . . . . . . . . . . . 88

A.5 Best fit parameters for galactic rotation curves. . . . . . . . . . . . . 88

B.1 WIMP scattering process. . . . . . . . . . . . . . . 90

D.1 The reciprocity theorem applied to diffusion. . . . . . . . . . . . . . 104

D.2 Diffusion in an annulus. . . . . . . . . . . . . . . . . . . . . 108

G.1 Layout for the copper baseplate of the experimental payload. . . . . . 144

G.2 Fiber focuser holder. . . . . . . . . . . . . . . . . . 145

G.3 Mirror holder. . . . . . . . . . . . . . . . . . . . . 145

G.4 Sample stage. . . . . . . . . . . . . . . . . . . . 146

G.5 Copper Enclosure. . . . . . . . . . . . . . . . . . 146

G.6 Crystal sample holder. . . . . . . . . . . . . . . . . . . . . 147

G.7 Crystal sample holder. . . . . . . . . . . . . . . . . . . . . . . 148

G.8 Break-out box for the ${ }^{3}$ He cryostat. . . . . . . . . . . . . . . . . . 149

G.9 Copper back plate for the cold amplifier. . . . . . . . . . . . . . . 150

G.10 Circuit board for cold amplifier. . . . . . . . . . . . . . . . . . 151

G.11 Component layout for cold amplifier. . . . . . . . . . . . . . . . . . . 152

G.12 Germanium crystal wafer used as source for crystal test samples. . . . 153

G.13 Full schematic for the amplifier circuit. . . . . . . . . . . . . . . . . . 154

G.14 Version 1 of the cold amplifier. . . . . . . . . . . . . . . . . . 155

G.15 Version 2 of the cold amplifier. . . . . . . . . . . . . . . . 156

G.16 Copper plate for version 3 of the cold amplifier. . . . . . . . . . . . 157

G.17 Circuit board for version 3 of the cold amplifier. . . . . . . . . . . . . 158

G.18 Version 3 of the cold amplifier. . . . . . . . . . . . . . . . . . . . 159

G.19 Circuit board for the warm amplifier. . . . . . . . . . . . . . . . 160

G.20 Circuit board for the control circuit. . . . . . . . . . . . . . . . 161 
G.21 Circuit board for the bias circuit. . . . . . . . . . . . . . 162

G.22 Poles and zeros for amplifier transfer functions. . . . . . . . . . 164

G.23 Noise spectrum of Sony 3SK165 MESFET. . . . . . . . . . . . 165

G.24 Photograph of experimental setup. . . . . . . . . . . 166

G.25 Temperature Control. . . . . . . . . . . . . . . . . . . 167

G.26 Detailed block diagram for the experiment control system. . . . . . 168

I.1 Equivalent circuit for piezo resonator. . . . . . . . . . . . . . 186 


\section{Chapter 1}

\section{Introduction}

The experiment described in this dissertation is a study of semiconductor physics under extreme conditions - specifically the transport of charge through ultra pure germanium crystals at cryogenic temperatures and in low electric fields. The fact that such an investigation of fundamental semiconductor physics is still necessary, even after half a century of industrial research on semiconductors, illustrates the exceptional nature of the conditions under which we wish to study these crystals.

The setting in which these unusual conditions arise, and to which this study is immediately applicable, is an experiment called the Cryogenic Dark Matter Search (CDMS). It is a fundamental physics experiment which aims to either prove or disprove some of the latest theories concerning the nature of a mysterious substance called dark matter, which makes up $26 \%$ of the mass of the universe, according to the latest astrophysical and cosmological studies.[1, 2, 3] The Cryogenic Dark Matter Search uses large, ultra pure, single crystals of germanium and silicon, held at cryogenic temperatures, as dark matter detectors.

The motivation for our charge transport experiment originated from an anomalous asymmetry observed between the behavior of electrons and holes in our germanium detector crystals. This asymmetry was found to be a consequence of the anisotropic propagation of electrons in germanium - an unexpected effect which only appears in the unusual combination of conditions (high purity, low temperature, and low electric field) under which our CDMS detectors are operated. 
This anomaly motivated the development of our Monte Carlo simulations of charge transport, which we used to confirm our explanation of the observed asymmetry. These computer models have subsequently proven to be extremely useful for both detector design and data analysis.

As our Monte Carlo simulations have grown to become indispensable tools for our investigations, the task of ensuring their accuracy has become imperative. Therefore, to refine our computer models, and to confirm their accuracy in detail, we designed and carried out the experiment described in this dissertation.

In order to provide the reader with the necessary context in which to understand both the motivation for this experiment and its applicability to CDMS, some background information regarding the current state of our knowledge of dark matter and its potential detection is provided in Chapter 2, and an overview of the theory of operation of our detector crystals is given in Chapter 3.

The anomalous effects which motivated the charge transport experiment are described in detail in Chapter 4, along with our efforts to explain these effects and analyze defective detectors using our Monte Carlo simulations.

Chapter 5 provides an overview of the theory of charge transport in semiconductors, and a brief description of our Monte Carlo models. More details can be found in reference [4], and a derivation of the phonon absorption and emission rates for free carriers in semiconductors is provided in Appendix F.

Chapters $6 \& 7$ describe the setup and methods for the charge transport experiment. The experimental results, and a discussion of their implications, are provided in Chapters $8 \& 9$. Chapter 10 summarizes the conclusions and provides a discussion of our plans for future work. 


\section{Chapter 2}

\section{Dark Matter and CDMS}

\subsection{Indirect Evidence for Dark Matter}

Since the 1930's evidence has accumulated for the existence of a new form of matter, called "dark matter", which does not interact with light.[5, 6] So far, the evidence for its existence has only come from its gravitational effects - namely the gravitational attraction of matter toward regions of space in which very little visible matter exists.

The first strong evidence that our universe might contain something other than ordinary matter came from measurements of the orbital velocities of stars and gas in galaxies. [7, 8] The relation between orbital velocity and orbital radius is called the galactic rotation curve, an example of which is plotted in Figure 2.1a.[9] Most galaxies follow the same pattern: orbital velocities do not approach zero at large orbital radius as would be expected from the visible mass distribution, but instead tend to asymptotically approach a constant value at large orbital radius.

The discrepancy can be explained by the presence of extra, invisible mass, called a dark matter halo, which surrounds and permeates the galaxy, and provides the extra gravity needed to keep the stars and gas gravitationally bound. However, this still leaves open the question of what comprises the dark matter, and why it should assume the particular density profile needed to give rise to the observed rotation curves. 


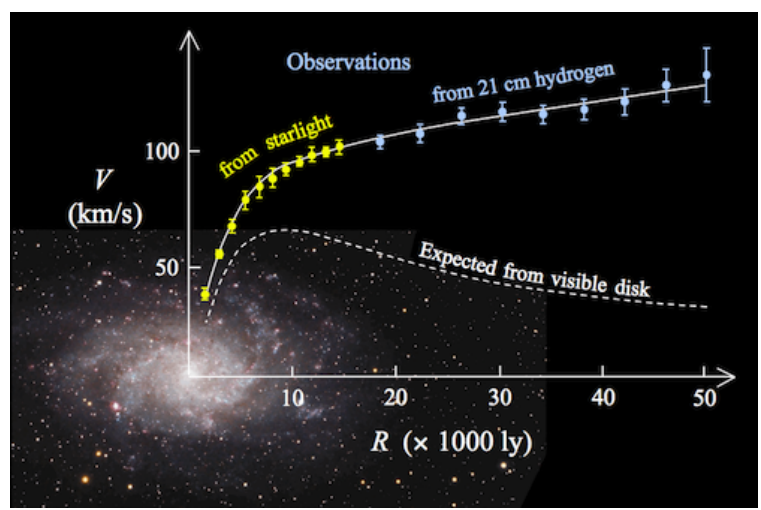

(a) Rotation curve for galaxy M33.[9, 10]

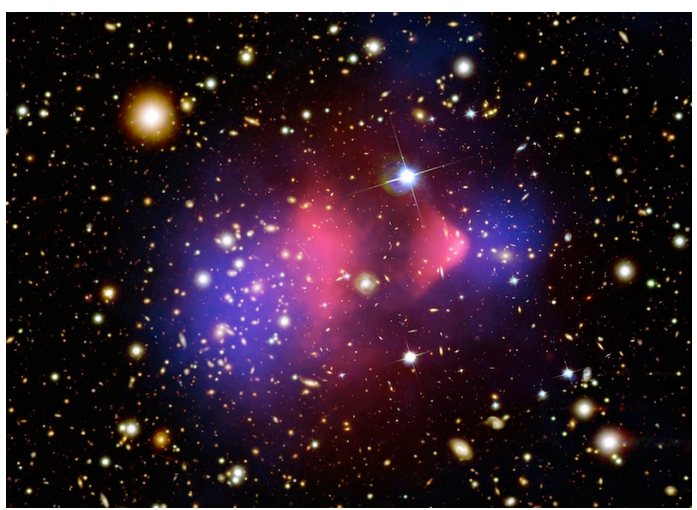

(b) The Bullet Cluster.[11]

Figure 2.1: Astrophysical evidence for dark matter. (a) Example of a galactic rotation curve.[9] The data points indicate the orbital velocity of gas and stars as a function of orbital radius, measured by Doppler shift. The dashed line indicates the predicted orbital velocity based on the visible matter alone. The addition of extra mass is required to make the model fit the data. (b) Modified image of the Bullet Cluster. The pink regions have been shaded to indicate the location of the visible matter in the galaxy cluster, measured by X-ray emission. The blue regions have been shaded to indicate the presence of mass, measured by gravitational lensing. The total mass of the galaxy cluster far outweighs the mass of visible matter, and it is located in a completely separate region.

It can be shown (see Appendix A) that a cloud of self-gravitating particles, in thermal and hydrostatic equilibrium, will naturally form a structure with an orbital velocity which approaches a constant value at large orbital radius, with the asymptotic velocity being proportional to the thermal velocity of the particles. Similar results have been predicted numerically from N-body simulations (see Figure 2.2). Therefore, if the hypothetical dark matter consists a new kind of fundamental uncharged particle, the galactic rotation curves can be explained in a natural and straightforward way.

Alternative theories have been proposed which instead try to explain galactic rotation curves by postulating modifications to the laws of gravity or inertia on galactic scales. $[13,14,15]$ Despite the success of these theories in the context of intra-galactic dynamics, a serious challenge to such ideas has arisen from recent observations of galaxy clusters, such as the Bullet Cluster in Figure 2.1b.[16] These data show a 


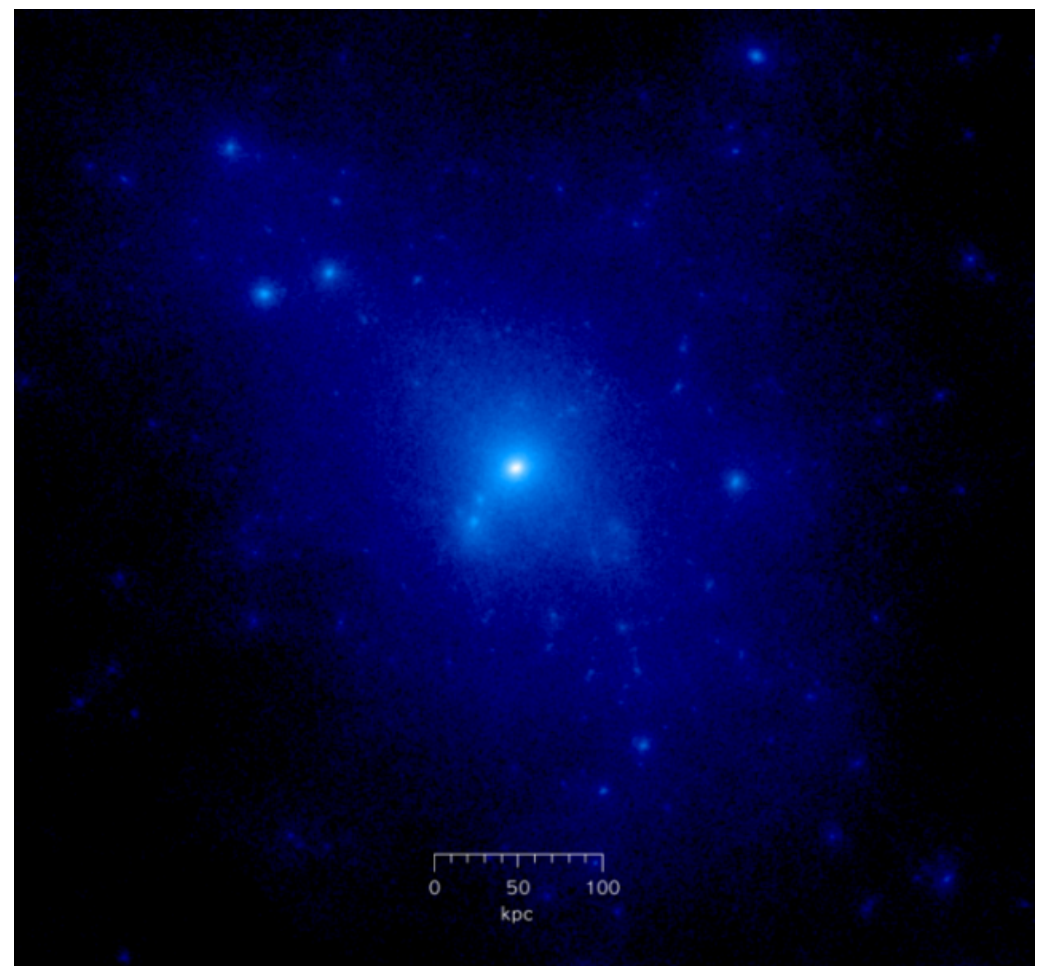

Figure 2.2: N-body simulation of a dark matter halo.[12] Scale indicated on bottom. Ordinary matter collects in the gravitational wells formed by these large structures and coalesces into what we see as the visible part of galaxies.

large spatial separation between the visible matter in a cluster and the major source of its gravitational mass, an effect which is difficult to reproduce through modifications to the laws of gravity or inertia alone.

Recently, additional evidence for dark matter has come from cosmology.[1, 2, 3] The power spectrum of fluctuations in the Cosmic Microwave Background (CMB) radiation are well fit by a model that requires a cold form of matter without electromagnetic interactions, i.e. dark matter. A plot of the CMB power spectrum is shown in Figure 2.3. 


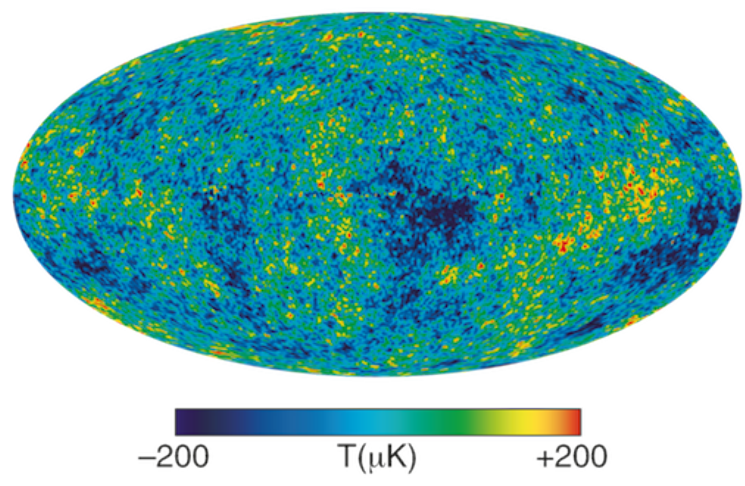

(a) CMB Temperature Fluctuations.[1]

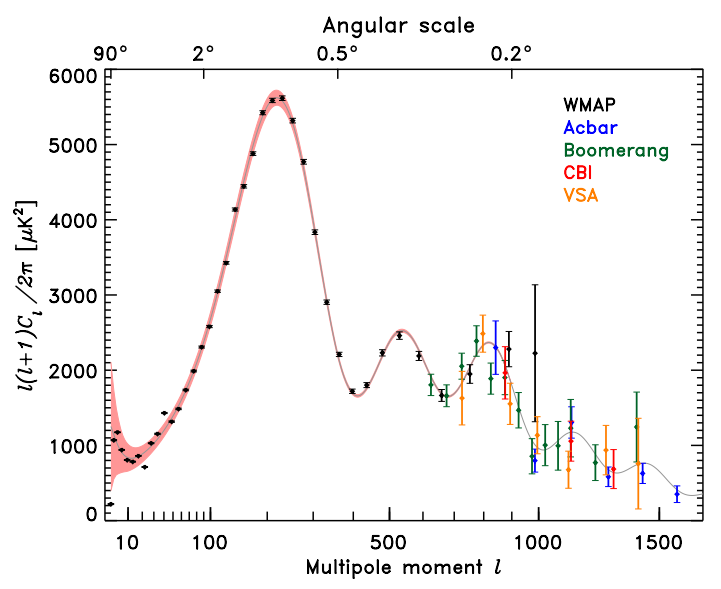

(b) Angular Power Spectrum.[17]

Figure 2.3: CMB Temperature Fluctuations. (a) Spatial fluctuations in the temperature of the CMB plotted on the surface of the celestial sphere with mean temperature subtracted. (Data from [1].) (b) Angular power spectrum of the temperature fluctuations. The power spectrum is extremely well fit by the $\Lambda$ CDM model, which contains a contribution from dark matter and dark energy in addition to the known particles of the standard model.

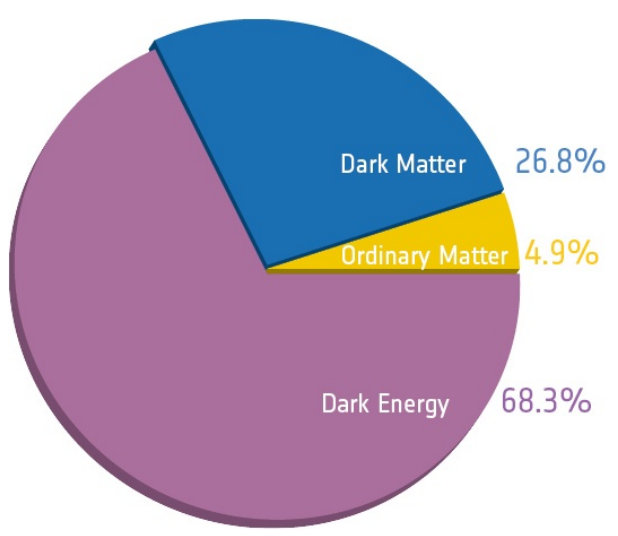

Figure 2.4: $\Lambda$ CDM model parameters from Planck, 2013 data release. [2, 18] According to this model, most of the mass of the universe comes from dark energy $(\Lambda)$ and cold dark matter (CDM). Ordinary matter comprises less than $5 \%$ of the mass of the universe. 


\subsection{Dark Matter Candidates}

Independent considerations in particle physics have led to extensions of the Standard Model which predict the existence of stable, uncharged fundamental particles.[19] One or more of these particles could potentially be what constitutes dark matter.

Most relevant to this dissertation is the Weakly Interacting Massive Particle (WIMP), a common product of supersymmetric extensions to the Standard Model.[20, 21] These hypothetical particles are predicted to have a mass somewhere between 1 and $10,000 \mathrm{GeV} / \mathrm{c}^{2}$, and a nucleon scattering cross-section between $10^{-44}$ and $10^{-52}$ $\mathrm{cm}^{2} \cdot[22]$

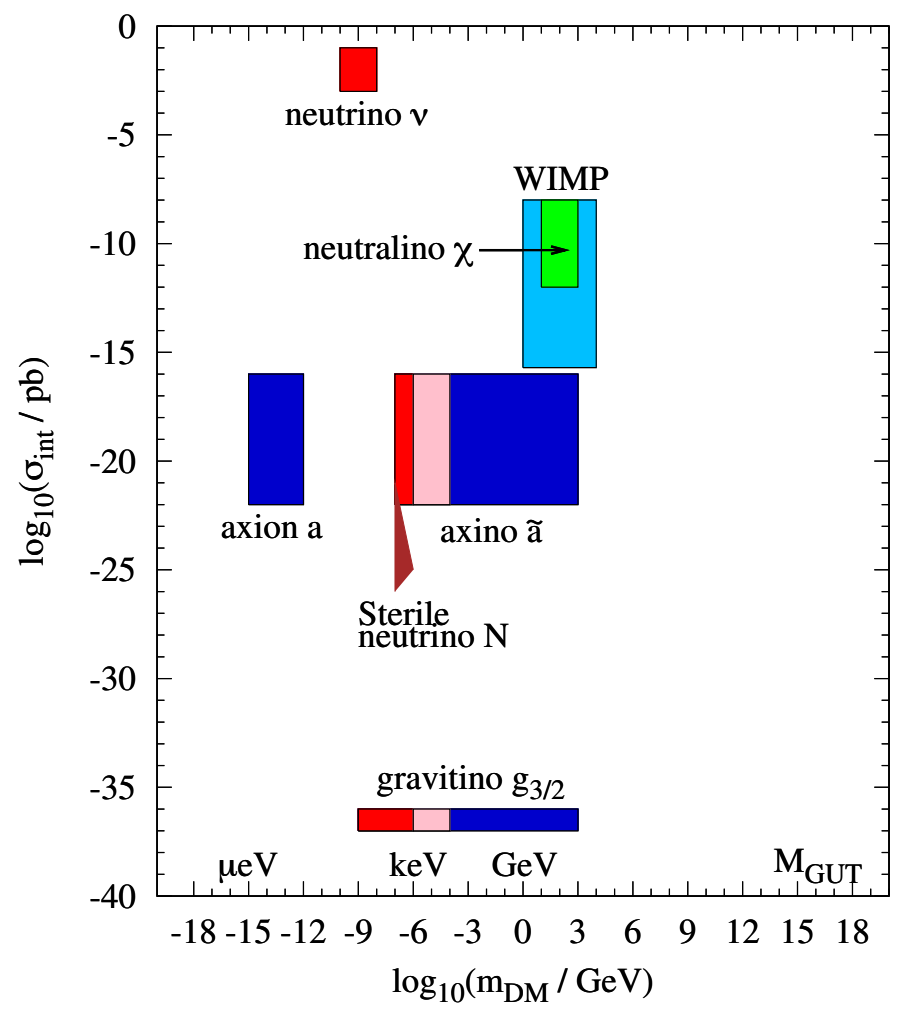

Figure 2.5: Hypothetical particles predicted by modern particle theory which could make up dark matter. [22] The plot shows the range of possible masses and interaction cross-sections allowed for each dark matter candidate. Weakly Interacting Massive Particles (WIMPs) and Axions are currently the most accessible dark matter candidates, and are hence the most actively studied. 


\subsection{Direct Detection of Dark Matter}

Because all forms of energy interact through gravity in an identical manner, the only way to differentiate between different dark matter candidates is by detecting a non-gravitational interaction between dark matter and ordinary matter. Figure 2.6 shows three methods for detecting non-gravitational interactions between ordinary matter and a dark matter particle, using a pseudo-Feynman diagram as a guide for categorizing the three types of possible interactions.[23, 24, 25, 26, 27]

The Cryogenic Dark Matter Search (CDMS) is an experiment which aims to directly detect the interaction between WIMPs and the atoms in a large, single crystal of germanium or silicon, illustrated by the red arrow in the diagram below.

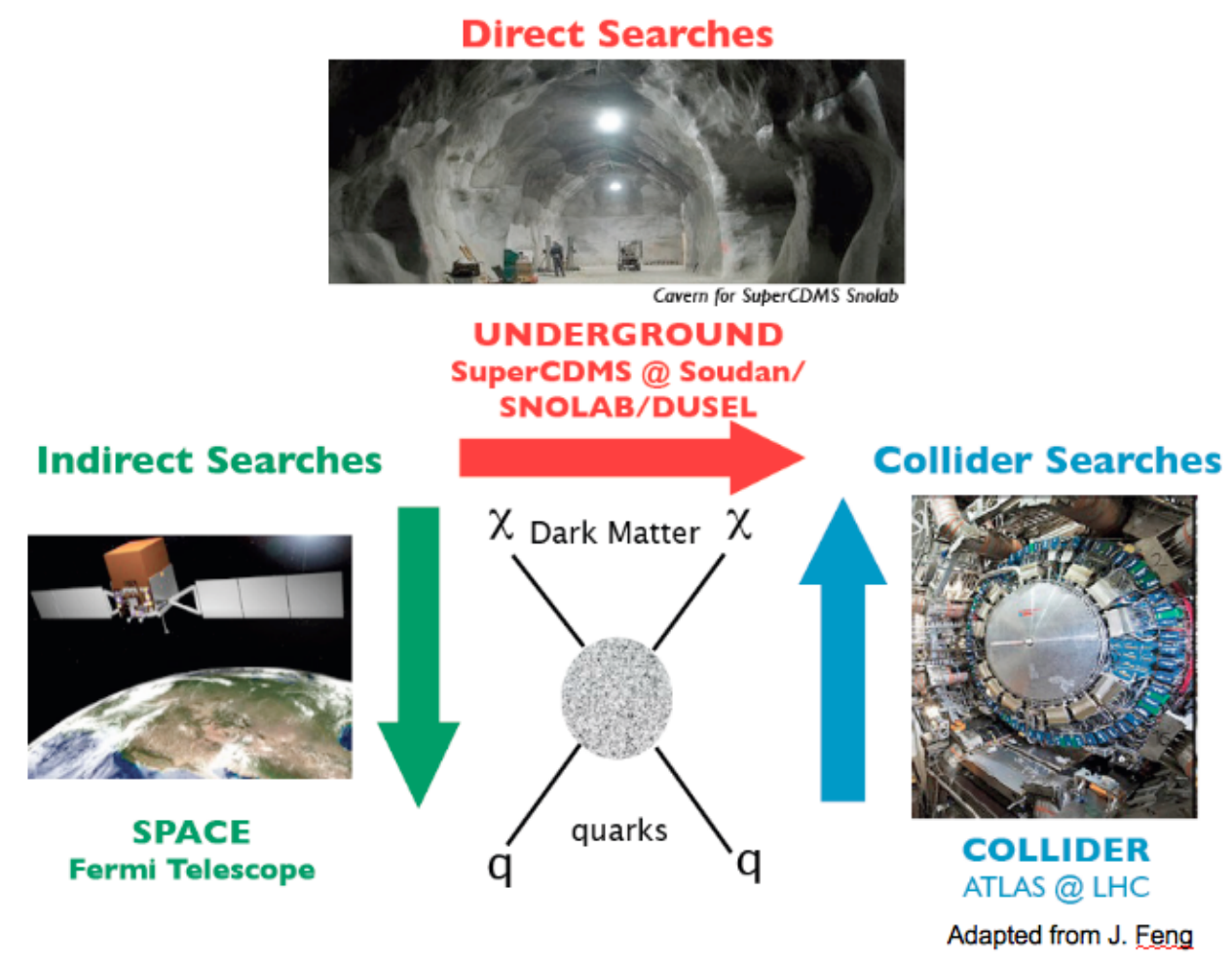

Figure 2.6: Non-gravitational searches for dark matter through 2-to-2 scattering. The arrows represent the direction of time in the pseudo-Feynman diagram for each type of interaction. The effective interaction point in the center represents a short-range interaction such as Z-boson exchange. 


\section{Chapter 3}

\section{CDMS Detector Physics}

\subsection{Particle Interactions}

Not much is currently known about the manner in which dark matter particles interact with ordinary matter. ${ }^{1}$ However, it is known that dark matter particles must be gravitationally bound to the dark matter halo, and therefore must travel with an orbital velocity comparable to that of all other stars and gas within the galaxy. ${ }^{2}$ Within the Milky Way, the asymptotic orbital velocity is about $220 \mathrm{~km} / \mathrm{s} .[29,30$, $31,32]$ We expect the dark matter particles in our vicinity to assume a MaxwellBoltzmann distribution with a velocity spread on the order of $220 \mathrm{~km} / \mathrm{s}$, centered around a drift velocity of $220 \mathrm{~km} / \mathrm{s}$ due to the orbital motion of our sun. ${ }^{3}$

Because these velocities are non-relativistic, the kinetic energy carried by the dark matter particles is simply $\frac{1}{2} m_{\chi} v^{2}$, where $m_{\chi}$ is the mass of the dark matter particle, and $v$ is its speed. For Weakly Interacting Massive Particles (WIMPs), the typical kinetic energy will be about $0.1 \mathrm{keV}$ for a particle of mass $1 \mathrm{GeV} / \mathrm{c}^{2}$, and $1,000 \mathrm{keV}$ for a particle of mass $10,000 \mathrm{GeV} / \mathrm{c}^{2}$.

\footnotetext{
${ }^{1}$ See the work by Kristi Schneck in [28] for a detailed study of all possible interaction modes between WIMPs and atomic nuclei.

${ }^{2}$ This is the primary reason why neutrinos cannot constitute dark matter. Due to their low mass, neutrinos travel with relativistic speeds at the known temperature of the neutrino background. Evidence for cold (i.e. non-relativistic) dark matter comes from cosmological observations. $[1,2,3]$

${ }^{3}$ See [33] for a more detailed model which accounts for Earth's orbital velocity.
} 


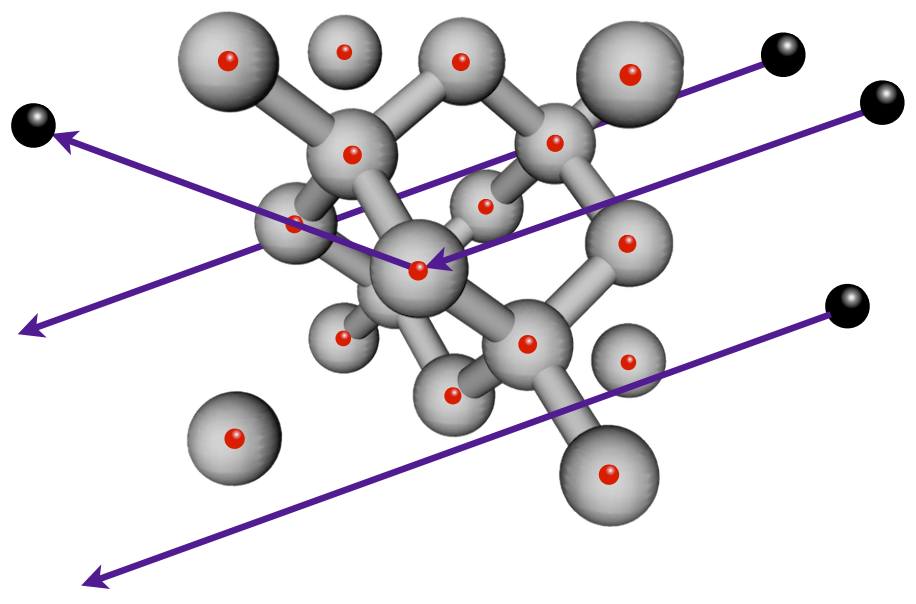

Figure 3.1: Interaction between a dark matter particle and a germanium nucleus. A continuous flux of dark matter particles (black) pass through the germanium crystal (gray). Occasionally a dark matter particle will interact with a germanium nucleus (red), rebounding elastically and transferring energy and momentum to the affected nucleus.

In the CDMS detector crystals, WIMPs can interact either with the electrons or the nuclei of germanium atoms. Interactions with electrons are not detectable because of the large mass mismatch between WIMPs and electrons which suppresses the WIMP scattering cross-section. ${ }^{4}$ In addition, scattered electrons can rebound with at most twice the velocity of the incident WIMP, at which speed they only carry a kinetic energy of about $0.5 \mathrm{eV}$. This is far below the threshold of detection. ${ }^{5}$

However, because the WIMP mass is predicted to be close to the mass of a typical atomic nucleus, the nuclei of the germanium atoms are capable of absorbing a substantial fraction of the kinetic energy of an incident WIMP. This energy is too small for the production of new particles or for the excitation of nuclear energy levels, so these interactions are expected to be purely elastic collisions.

Following reference [33], we may estimate the rate of interaction, $r$, between a single atomic nucleus and the flux of WIMPs as:

\footnotetext{
${ }^{4}$ At non-relativistic speeds, the interaction cross-section between a WIMP and a particle of ordinary matter is proportional to $\mu^{2}$, where $\mu$ is the reduced mass of the pair. (See appendix B for further discussion).

${ }^{5}$ Current detector thresholds are $100 \mathrm{eV}$ for phonons and $1 \mathrm{keV}$ for charge.[34]
} 


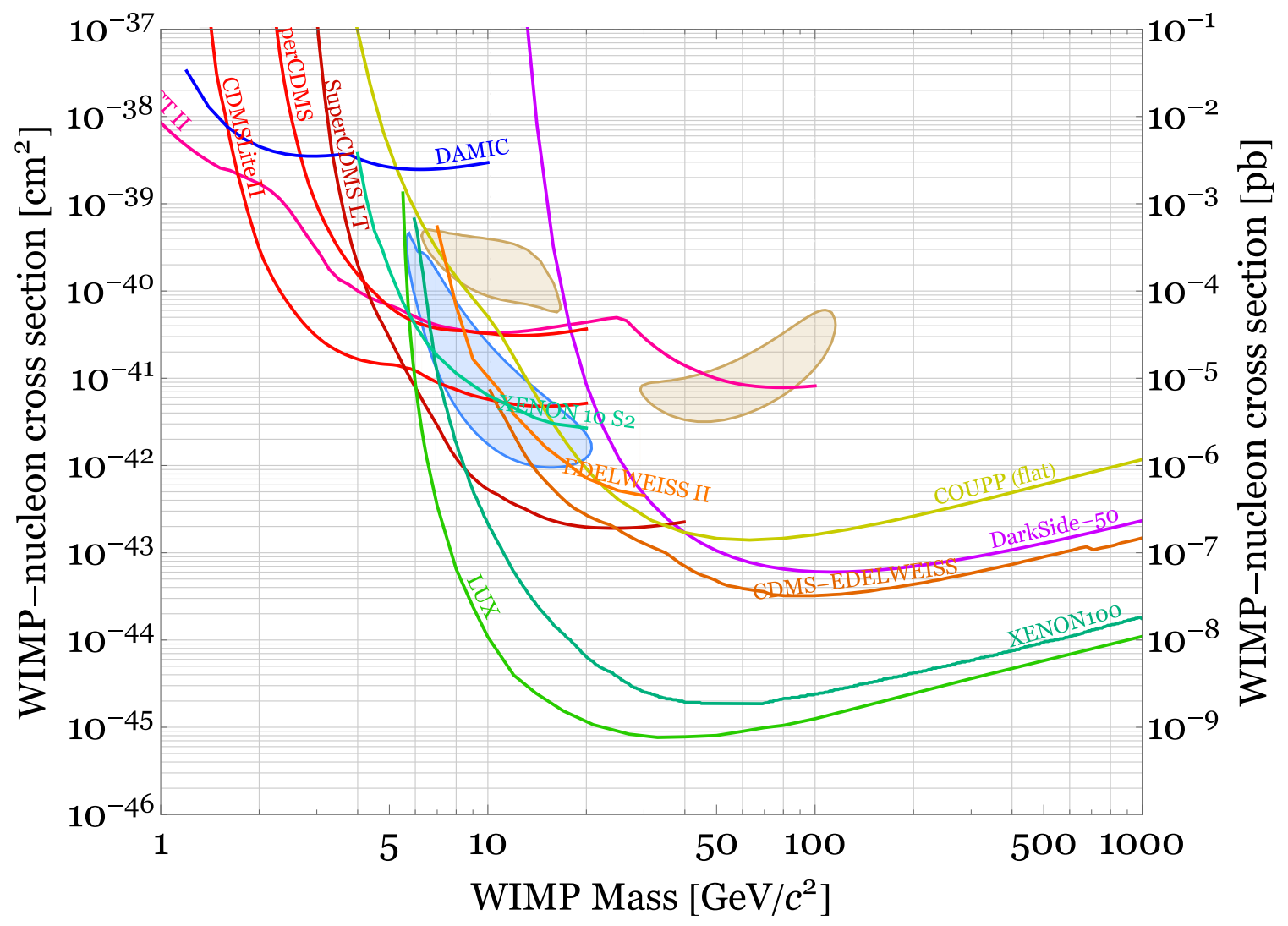

Figure 3.2: Current limits on the WIMP interaction cross-section from multiple experiments as a function of the WIMP mass. [35] Interaction strengths above the limit curves are excluded.

$$
r=n v \sigma_{0} A^{2} \frac{\mu^{2}}{\mu_{0}^{2}}
$$

where $n$ is the number density of WIMPs, $v$ is the mean WIMP speed, $\sigma_{0}$ is the WIMP-nucleon scattering cross-section, $\mu$ is the reduced mass of the nucleus, $\mu_{0}$ is the reduced mass of a nucleon, and $A$ is the atomic mass number of the nucleus. ${ }^{6}$

\footnotetext{
${ }^{6}$ The scattering rate is enhanced by a factor of $A^{2}$ rather than $A$ because the WIMP wavefunction scatters coherently from all of the nucleons within the atomic nucleus. This is a consequence of the fact that the interaction energy is not large enough to excite nuclear energy levels. The final state therefore contains no information about which nucleon the WIMP scattered from. (See appendix B for further discussion.)
} 
The number density, $n$, of WIMPs can be estimated from the halo mass density, $\rho$, using the relation $\rho=n m_{\chi}$, where $m_{\chi}$ is the WIMP mass. A good approximation (see Appendix A, Equation A.10) for the mass density at the location of the sun is:

$$
\rho=\frac{v_{0}^{2}}{4 \pi G a^{2}}=0.5 \frac{\mathrm{GeV} / \mathrm{c}^{2}}{\mathrm{~cm}^{3}}
$$

where $G$ is Newton's constant, $v_{0}=220 \mathrm{~km} / \mathrm{s}$ is the asymptotic orbital velocity in the Milky Way, and $a=8.3 \mathrm{kpc}$ is the orbital radius of the sun in the Milky Way.[36, 37, 38]

Let us consider the case of a WIMP with a mass of $100 \mathrm{GeV} / \mathrm{c}^{2}$. If such a particle exists, its scattering cross-section has been shown by CDMS to be less than $2 \cdot 10^{-44}$ $\mathrm{cm}^{2}$. (See Figure 3.2.) The average rate of WIMP collisions experienced by a single germanium nucleus can be roughly estimated to be:

$$
r=\frac{\rho}{m_{\chi}} v \sigma_{0} A^{2} \frac{\mu^{2}}{\mu_{0}^{2}}<2.5 \cdot 10^{-32} / \mathrm{s}
$$

where $^{7}$

$$
\begin{aligned}
A & =73 \\
m_{\chi} & =100 \mathrm{GeV} / \mathrm{c}^{2} \\
m_{\mathrm{Ge}} & =73 \mathrm{amu} \\
\mu & =1 /\left(1 / m_{\chi}+1 / m_{\mathrm{Ge}}\right)=43.4 \mathrm{amu} \\
\mu_{0} & =1.0 \mathrm{amu} \\
v & =250 \mathrm{~km} / \mathrm{s} \\
\sigma & <2 \cdot 10^{-44} \mathrm{~cm}^{2}
\end{aligned}
$$

The rate per unit mass, $R$, may be calculated by dividing $r$ by the mass of a single germanium atom:

$$
R=\frac{r}{m_{\mathrm{Ge}}}<\frac{0.02}{\mathrm{~kg} \text { day }}
$$

$$
{ }^{7} v=\int d^{3} v^{\prime}\left|v^{\prime}\right|\left(2 \pi v_{\mathrm{T}}^{2} / 3\right)^{-3 / 2} e^{-\frac{\left(v^{\prime}-v_{0}\right)^{2}}{2 v_{\mathrm{T}}^{2} / 3}}
$$


During the last run of SuperCDMS, lasting from 2012 to 2014, the total detector exposure, $E$, was about $600 \mathrm{~kg}$ days.[39] During this run, the expected number of dark matter events, $N$, generated by a $100 \mathrm{GeV} / c^{2}$ WIMP, with interaction cross-section $\sigma_{0}<2 \cdot 10^{-44}$, was:

$$
N=R E<10
$$

which is consistent with the number of events generated by known background processes. At a mean energy of $10 \mathrm{keV}$ per interaction, this corresponds to an average radiation dose rate of less than $10^{-14}$ gray/yr.

We therefore see that dark matter interactions are quite rare, making long exposure times necessary. The difficulty of dark matter detection is further exacerbated by its exceedingly low dose rate compared to natural background radiation at Earth's surface, which is roughly $10^{-3}$ gray/yr, or $10^{11}$ times higher than the dose rate from dark matter interactions.[40]

For a more detailed calculation of the expected interaction rates in our detectors, see references [28] and [33].

\subsection{Energy Collection}

CDMS detectors consist of large, ultra pure, cylindrical, single crystals of germanium or silicon (see Figure 3.3). The top and bottom surfaces of these crystals are instrumented with sensors capable of detecting free charges and phonons.

When an incident particle impacts an atom within the detector, its deposited energy excites the atoms within a small region of the crystal. These high-energy excitations eventually down-convert into more numerous low-energy excitations until all of the deposited energy is contained in three forms: free charge carriers, phonons, and crystal defects, the crystal defects containing no more than about $3 \%$ of the total energy.[41]

Electrodes on the surface of the crystal create an electric field in the bulk which separates the free electrons and holes and drifts them toward the two surfaces. When these charges are collected on the surface electrodes, they cause a small change in 

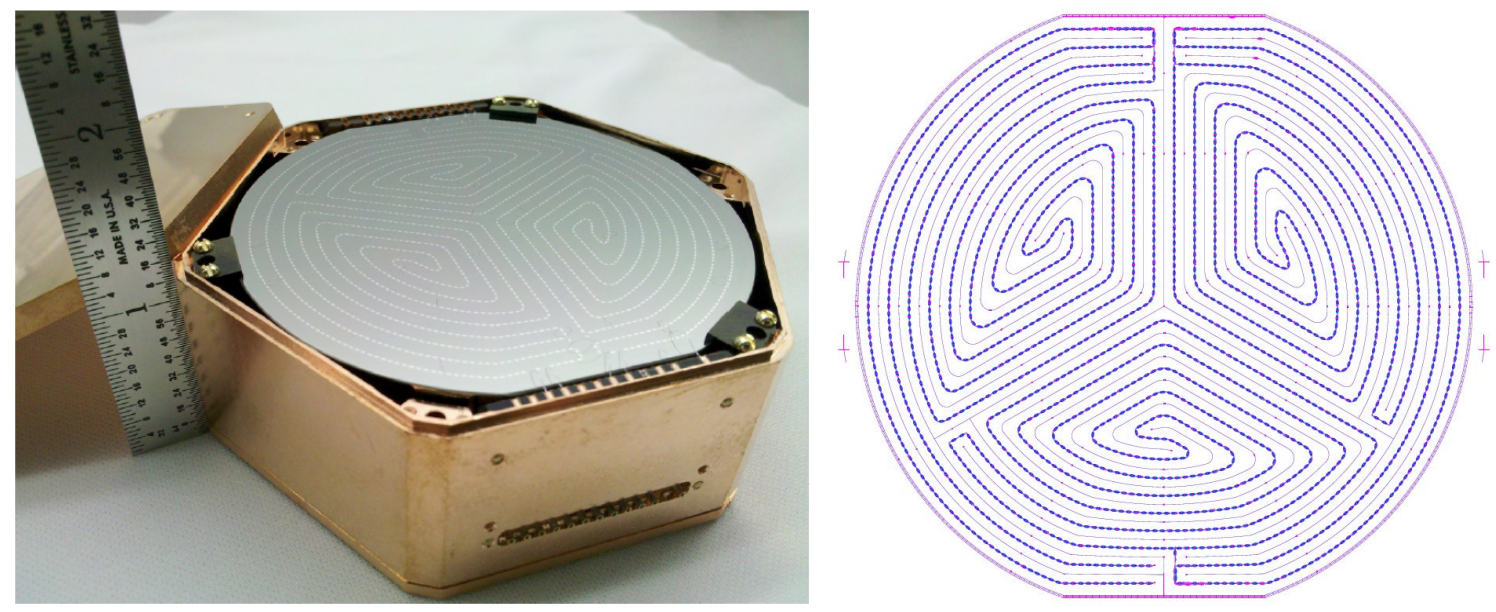

Figure 3.3: CDMS iZIP (Interleaved Z-sensitive Ionization and Phonon) detector. Left: Photograph of detector in copper housing. Right: iZIP surface pattern.

voltage of each electrode, which is amplified and measured.

The surface also contains multiple small transition-edge sensors (TES's), consisting of small wires of tungsten biased at their superconducting transition temperature of $70 \mathrm{mK}$. These sensors are sensitive to incident phonons, which raise the temperature of the tungsten, causing a significant and measurable change in its electrical resistance.

The transition-edge sensors on the crystal surface detect both the phonons generated directly by the incident particle (primary phonons), as well as phonons emitted by the free charge carriers as they fall through the potential gradient in the crystal bulk (Luke phonons). Figure 3.4 illustrates this complete process.

The analysis of phonon diffusion is complicated by the fact that the mean-freepath of phonons is wavelength-dependent, with longer-wavelength phonons having longer mean-free paths. ${ }^{8}$ In addition, the phonons down-convert to lower frequencies as they propagate through the crystal due to non-linearities in the crystal elasticity, causing the mean-free-path to grow. Therefore, there will always come a point at

\footnotetext{
${ }^{8}$ Primary phonons have energies close to the Debye frequency when they are first created, and therefore initially have a very short mean-free-path. Luke phonons have much lower frequencies and are balistic.
} 


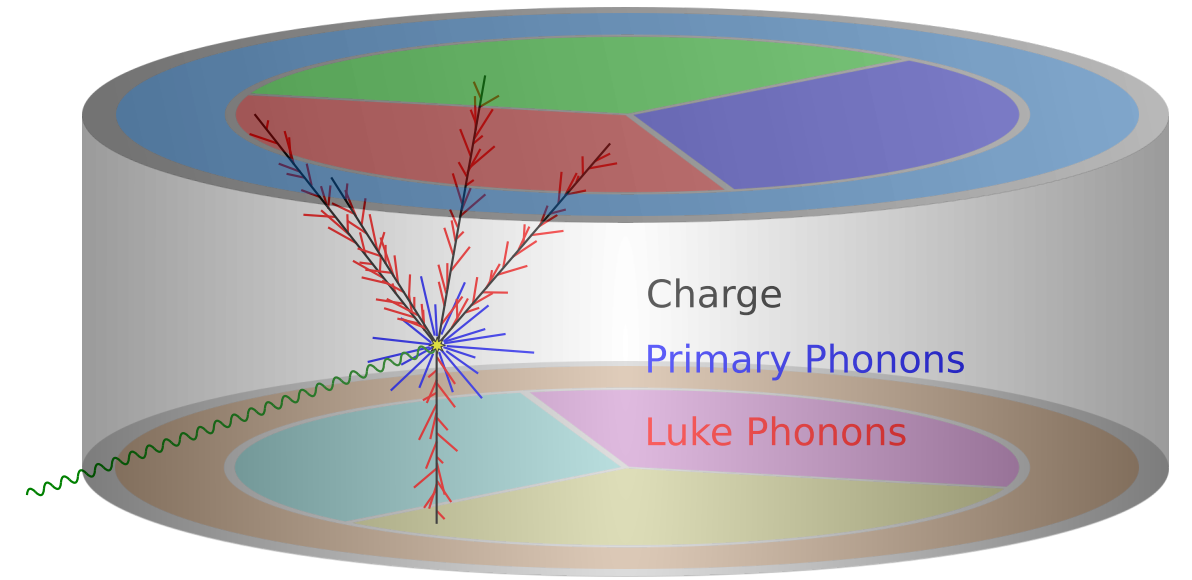

Figure 3.4: The result of a localized event of energy deposition by an incident gamma ray (green) in a CDMS iZIP detector crystal (gray). Trajectories of charge carriers are shown in black. Trajectories of primary and Luke phonons are shown in blue and red, respectively. Phonon trajectories are truncated to reduce visual clutter.

which the mean-free-path is comparable to the crystal size. At this point, the phonon transport is poorly modeled as either a purely diffusive or a purely ballistic process. Appendix C provides a model for phonon transport in this quasiballistic regime.

In order to enhance the collection efficiency of the transition-edge sensors, the tungsten wires are attached to short superconducting aluminum collection fins, aluminum having a superconducting transition temperature of approximately $1 \mathrm{~K}$. These fins absorb incident phonons which break Cooper pairs, generating unpaired charges known as quasiparticles. These quasiparticles diffuse through the aluminum until they reach the tungsten, at which point they are absorbed. Appendix D provides a detailed calculation of this diffusion and absorption process. For an experimental study of this diffusion process, see the work by Jeff Yen in reference [42].

Appendix E provides a derivation of the linear response of a transition-edge sensor to an impulse of heat power. For the case in which the transition-edge sensor becomes non-linear, it becomes necessary to use a non-linear template-fitting method to estimate the absorbed energy. For further discussion of this method, see the work by Ben Shank in reference [43]. 


\subsection{Shielding and Discrimination Power}

As discussed in $\S 3.1$, known background sources produce a radiation dose rate at least $10^{11}$ times larger than the dose rate expected from potential dark matter interactions. This large discrepancy makes both shielding and background discrimination essential to any unambiguous detection of dark matter.
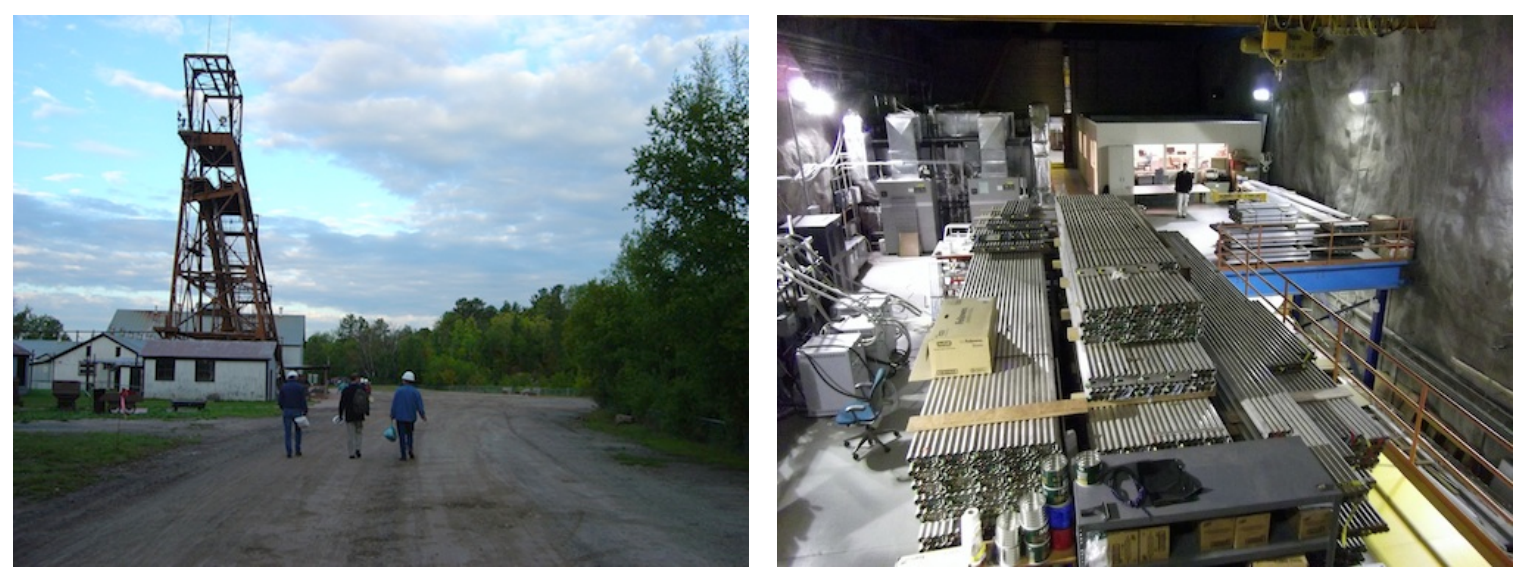

Figure 3.5: Left: Headframe for the Soudan Mine (photo courtesy of Dan Bauer). Right: Cavern in the Soudan Mine containing the CDMS experiment.

In order to reduce its exposure to background radiation, the CDMS experiment is conducted at a depth of $2341 \mathrm{ft}$ in the Soudan Mine in northern Minnesota, which provides protection from high-energy cosmic rays. (See Figure 3.5.) Further shielding is achieved through the use of passive layers of lead and polyethylene, and an active veto signal generated by a layer of muon-sensitive scintillator surrounding the experimental apparatus. (See Figure 3.6.) Together, these measures reduce the background rate by about six orders of magnitude.

A further reduction by a factor of about $10^{5}$ is achieved by discriminating between different types of observed events and rejecting those events which show a signature characteristic of background sources. This type of discrimination is made possible by the fact that CDMS detectors measure two forms of energy: both phonons and charge. Different types of particle interactions will distribute their energy differently between the phonons and charge. Particles that interact primarily with electrons, 


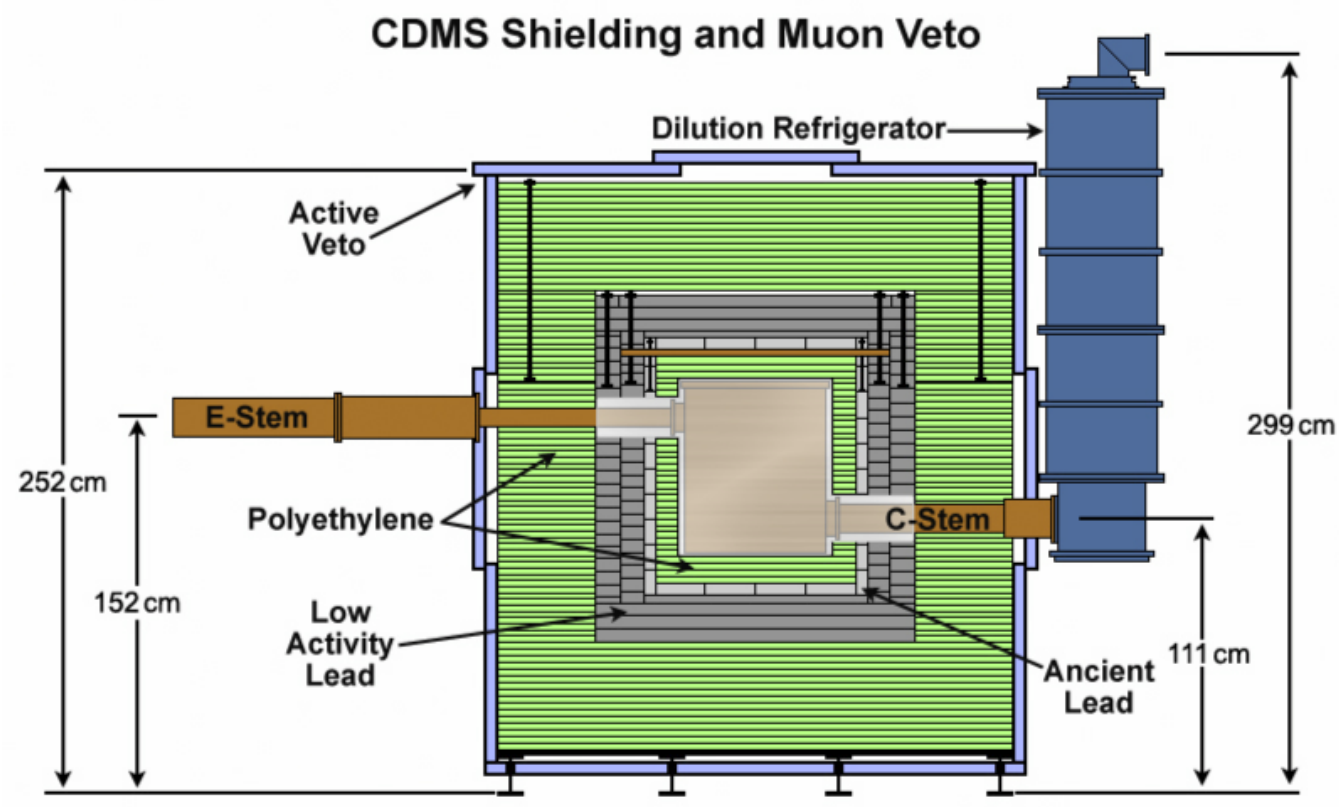

Figure 3.6: Diagram of the CDMS experimental apparatus showing lead and polyethylene shielding, along with the active scintillator panels.

such as gamma and beta radiation, distribute more of their energy into the production of electron-hole pairs, while particles that interact primarily with atomic nuclei, such as neutrons and hypothetical WIMPS, distribute more of their energy into the production of primary phonons.

Therefore, the dimensionless ratio of charge energy to phonon energy, called the "ionization yield", provides a good indication of the type of particle interaction. Figure 3.7 shows a plot of two populations of events: electron-recoil events are plotted in blue, while nuclear-recoil events are plotted in red. Notice how the ionization yield clearly separates the two populations.

This type of discrimination power is vitally important for any low-background experiment like CDMS which must look for extremely rare events which occur at a rate much lower than known sources of background radiation. 


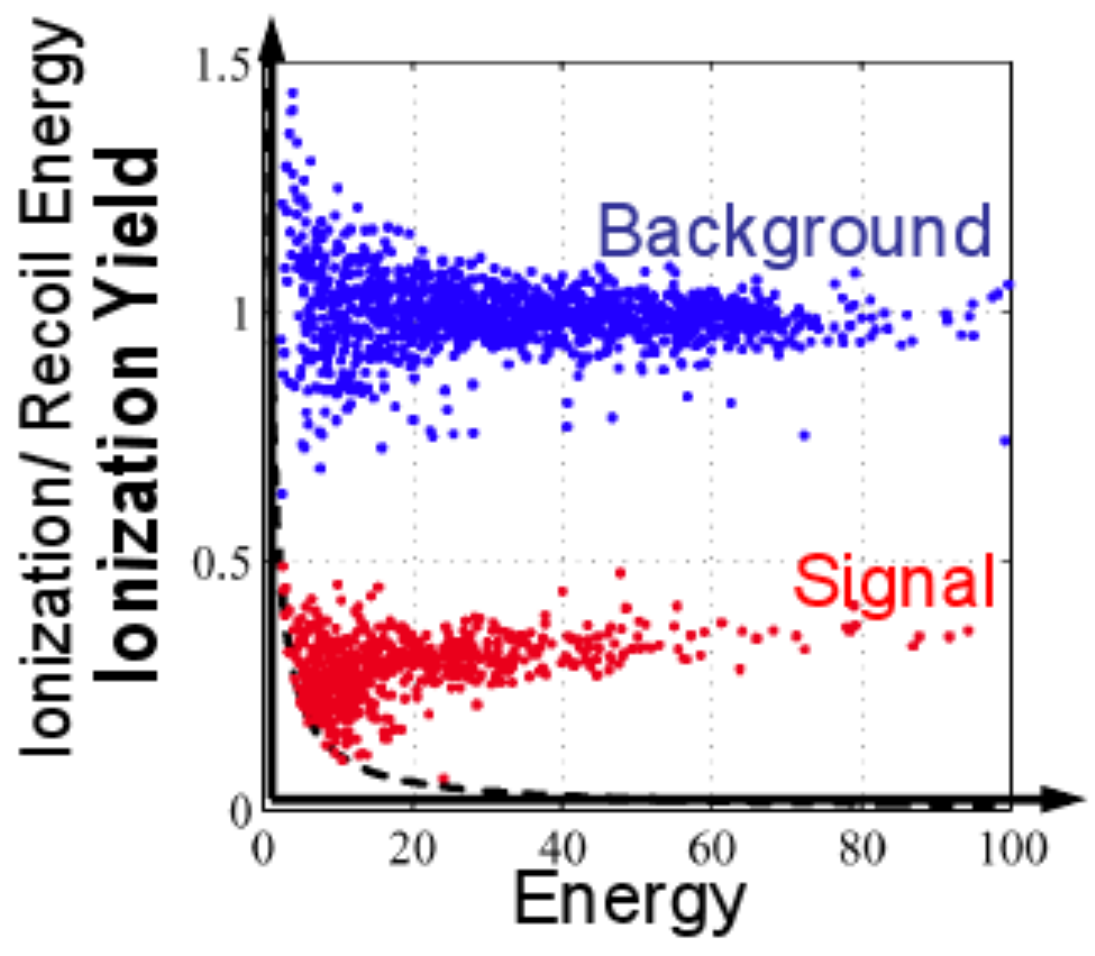

Figure 3.7: CDMS detector background discrimination. Vertical axis represents the yield, defined to be proportional to the ratio of charge to phonon energy, normalized such that electron-recoil events have a yield of 1 . The horizontal axis represents the sum of electron and phonon energy. The population of electron-recoil (background) events is colored blue, while the nuclear-recoil (signal) events are colored red. The signal events in this case were provided by a neutron calibration source. 


\section{Chapter 4}

\section{Anomalous Effects in CDMS Crystals}

\subsection{Charge Sharing Asymmetry}

As described in Chapter 1, an unexpected asymmetry between electron and hole propagation was observed in several CDMS crystals.[44] One such example was a dislocation-free germanium crystal from E. Haller, which was being used to measure the rate of charge trapping in germanium crystals without dislocations at cryogenic temperatures.[45]

The Haller crystal was biased and exposed to gamma radiation from an ${ }^{241} \mathrm{Am}$ source. Charges were collected by two cylindrically-symmetric electrodes on the top of the crystal. The geometry of the electrodes and the internal electric field for the Haller crystal are shown in Figure 4.1.

It was originally assumed that any free carriers generated within the bulk of the crystal would be drifted parallel to the electric field lines until they reached the electrodes at the surface. This would imply that charges could only be shared between inner and outer electrodes if the point of interaction occurred exactly on the border which separates the region containing electric field lines which end on the inner electrode from the region containing electric field lines which end on the outer electrode. An interaction which occurred at any other location would distribute all 


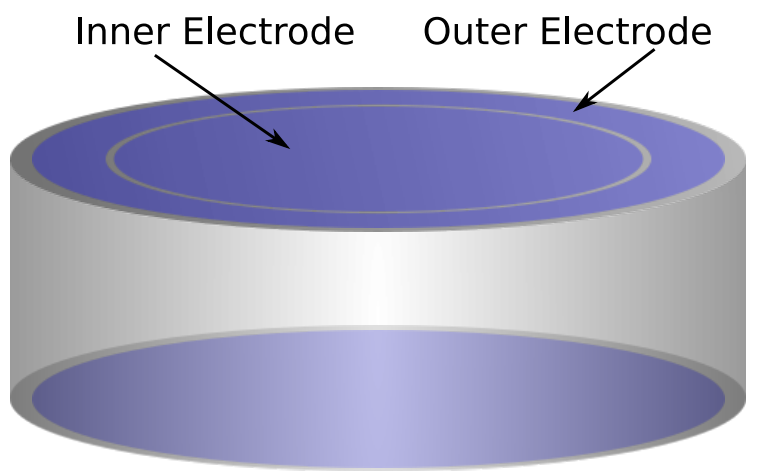

(a) Charge channels on Haller crystal.

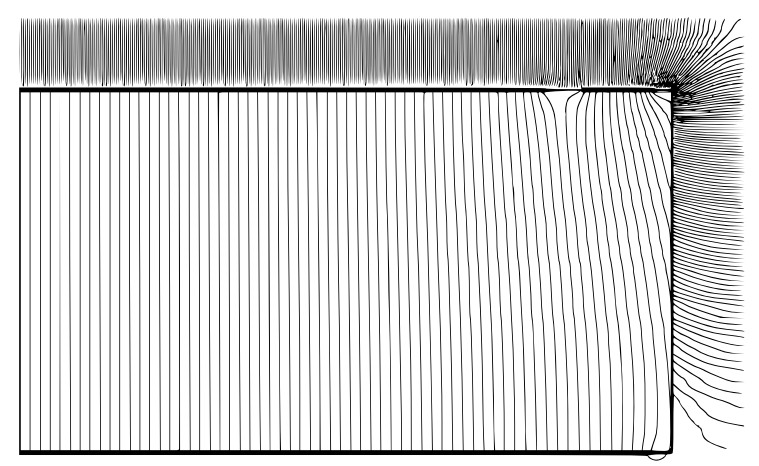

(b) Cross section showing E-field.

Figure 4.1: Illustration of the crystal geometry used in the tests of charge sharing between electrodes in the Haller dislocation-free crystal.

of its charge either on the inner or on the outer electrode.

However, this is not what was seen in the experimental data. Figure 4.2 shows plots of the charge event energy vs. the charge-sharing parameter. It was expected that very few events would have a charge-sharing parameter with a value other than +1 or -1 . However, when the crystal was under positive bias, a large population of events appeared which had a substantial amount of shared charge between the inner and outer electrode.

This effect was not only observed in the Haller dislocation-free crystal, but also in the germanium CDMS II detectors [47], and the germanium SuperCDMS iZIP detectors [46]. Figure 4.3 shows plots of the yield (charge energy divided by phonon energy) for the outer vs. the inner electrode on one side of a SuperCDMS iZIP detector. Unshared events occur along the axes, while shared events occur in the middle of the plot. Note the large populations of shared events when electrons are collected by the electrodes.

Unlike the Haller crystal and the CDMS II detectors, the SuperCDMS iZIP detectors are symmetric, having split inner and outer electrodes and charge amplifiers on both sides. By reversing the bias across the crystal, the charge sharing was clearly shown to be associated with electron propagation, and not with an asymmetry in the detector design. 


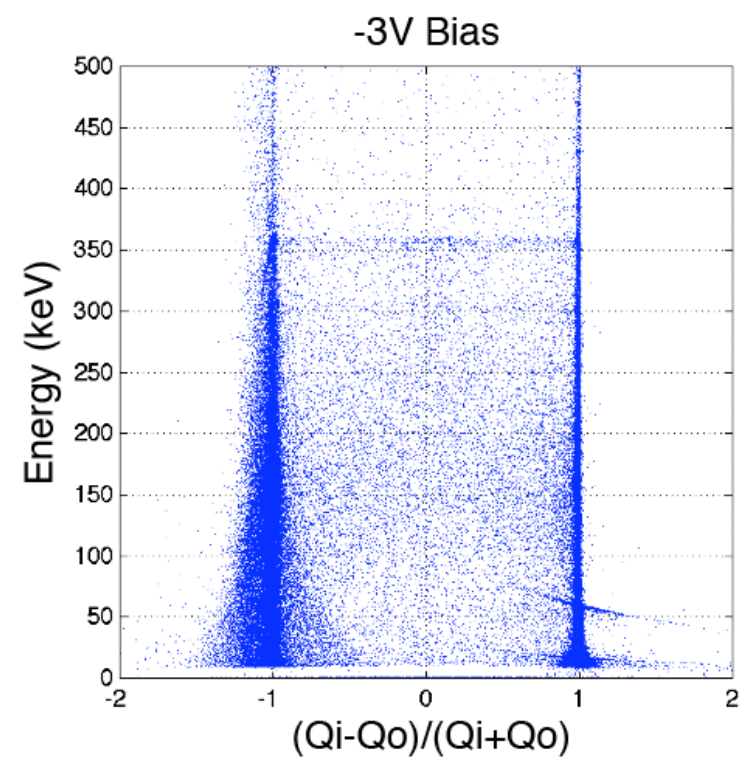

(a) Hole propagation.

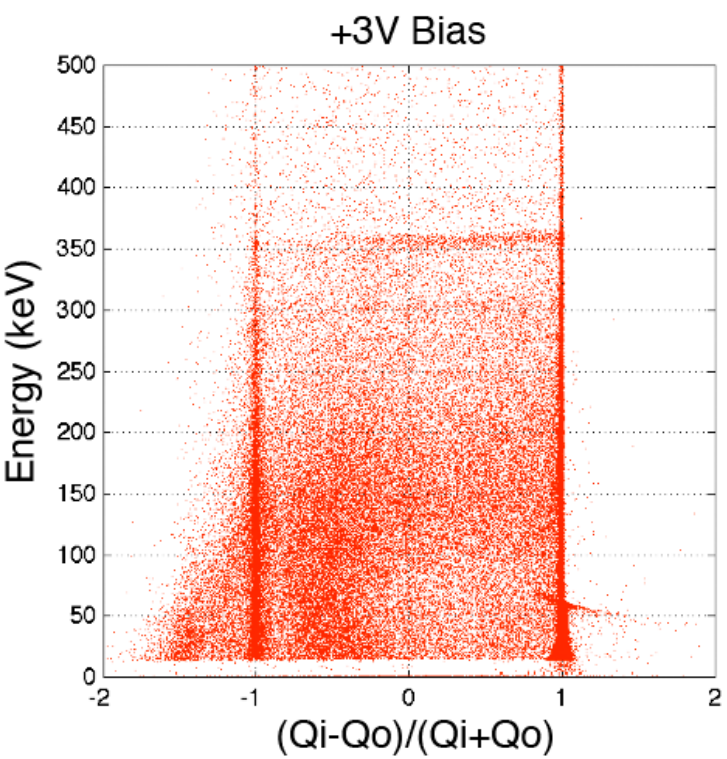

(b) Electron propagation.

Figure 4.2: Charge partitioning between inner and outer channels for positive and negative bias. Note that charge is shared between electrodes much more frequently under positive bias. [44]

The correlation of this effect with electron propagation was the key to its explanation. The effect comes from the anisotropy of electron transport due to the structure of the conduction band in germanium. The effect is only noticeable at low temperature and low electric field in crystals of high purity. A simple Monte Carlo simulation, shown in Figure 4.4, demonstrates how the anisotropy of electron propagation can generate shared events for electrons but not for holes.

The details of this anisotropy, and the conditions under which it is important, are discussed in Chapter 5. 


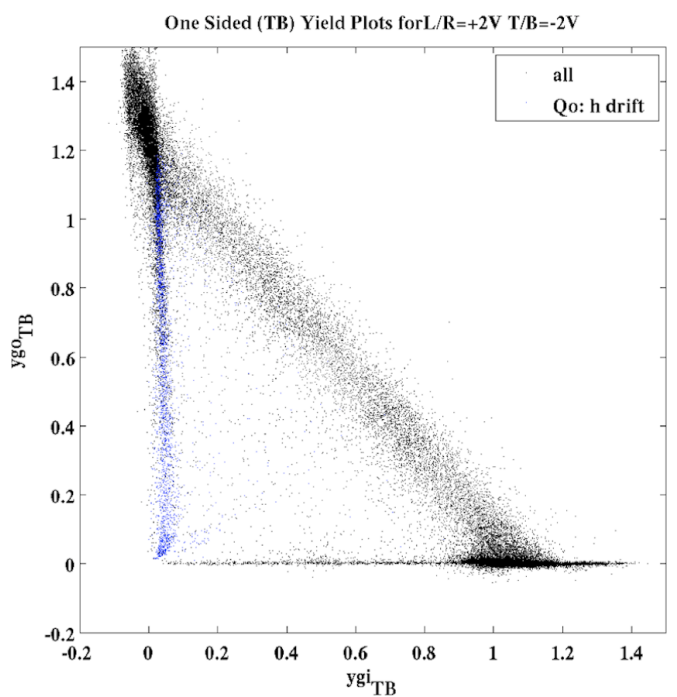

(a) Hole propagation.

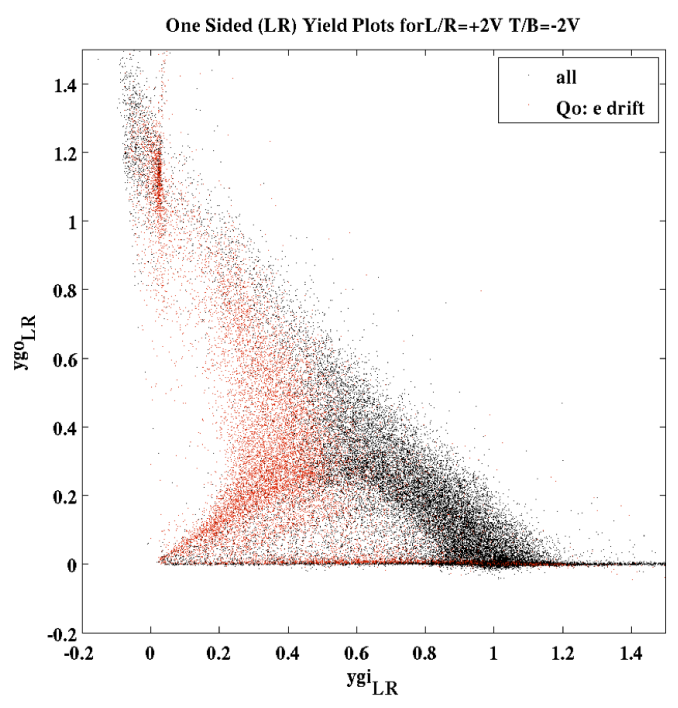

(b) Electron propagation.

Figure 4.3: Yield plots (charge energy divided by total phonon energy) for the outer vs. the inner electrodes of a SuperCDMS iZIP detector under two different bias conditions. Points falling on the axes represent events in which one or the other electrode collected no charge. Points in the middle of the plot represent events in which some charge arrived on both electrodes. Note the large population of events which share charge between both electrodes when electrons are collected (b) vs. the low number of shared events when holes are collected (a). Note also that some events in (b) have a low total yield. These represent events which occurred near the edge of the crystal in which not all charge was collected. This occurs with a higher frequency in the case of the electrons because of their oblique propagation.[46] 

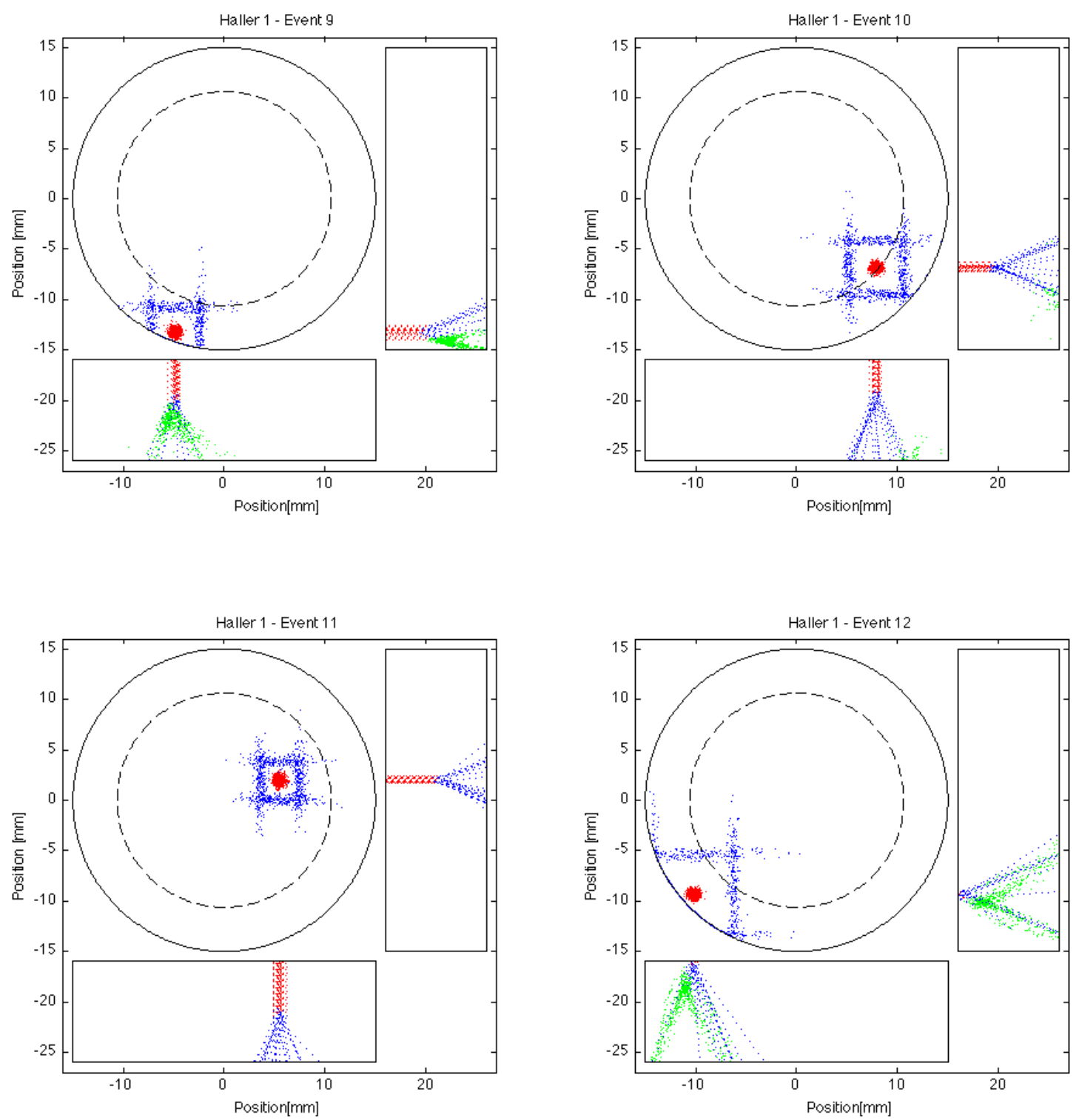

Figure 4.4: Three-dimensional Monte Carlo simulation of oblique electron propagation through the Haller crystal, projected along $\mathrm{x}, \mathrm{y}$, and $\mathrm{z}$ directions. The electrons (blue) travel obliquely to the applied electric field and separate into four clusters. The holes (red) travel parallel to the applied electric field and remain in a single tight cluster.[44] 


\subsection{Shorted Charge Channel}

In the SuperCDMS experiment, one of the detectors ${ }^{1}$ developed a short in one of the outer charge-collection electrodes ${ }^{2}$. This modified the electric field pattern within the crystal bulk, as illustrated in Figure 4.5 a\&b.

Monte Carlo simulations, shown in Figure $4.5 \mathrm{c} \& d$, revealed that events near the shorted electrode allowed electrons to propagate to the inner electrode due to the modified electric field and the oblique propagation of the electrons. The distribution of charge from such events would subsequently give a false estimation of the radial coordinate of the event location. This is especially problematic due to the fact that events near the surfaces of CDMS crystals are regularly cut from the dataset due to their high probability of coming from radioactive surface contamination.

See the dissertation by Kristi Schneck (reference [28]) for a detailed analysis of the behavior of the shorted charge channel.

\subsection{Importance of Accurate Modeling}

Both of the anomalous effects discussed in this chapter illustrate the importance of having an accurate Monte Carlo model to simulate the behavior of the CDMS detector crystals. Such a model is useful for understanding the behavior of detectors, as well as for diagnosing problems.

\footnotetext{
${ }^{1}$ Detector T5Z3.

${ }^{2}$ Channel QOS1.
} 


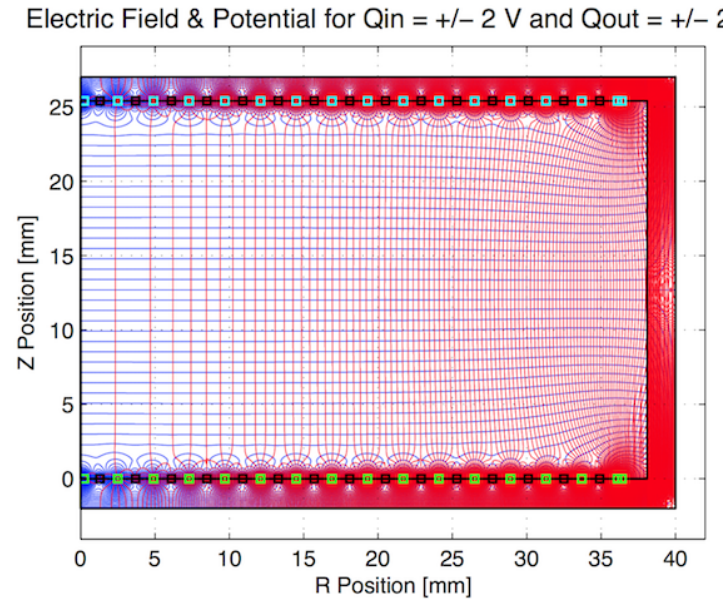

(a) E-field in normal detector.

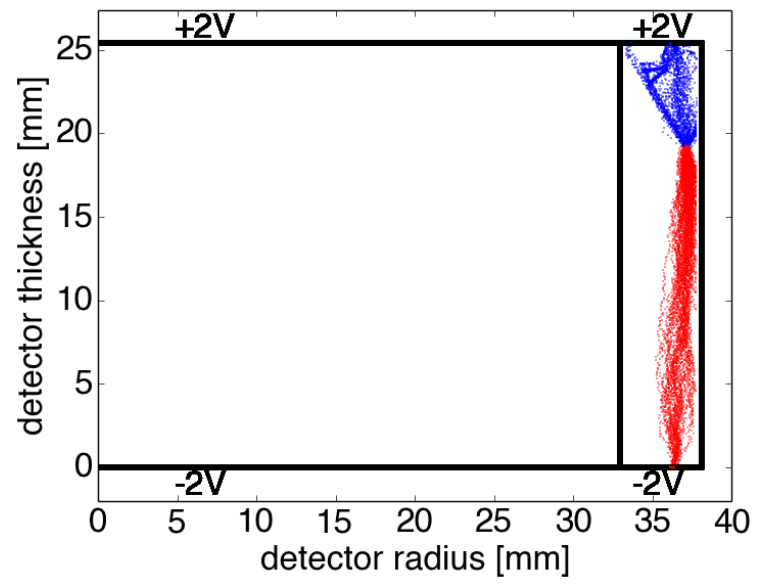

(c) Chage propagation in normal detector.

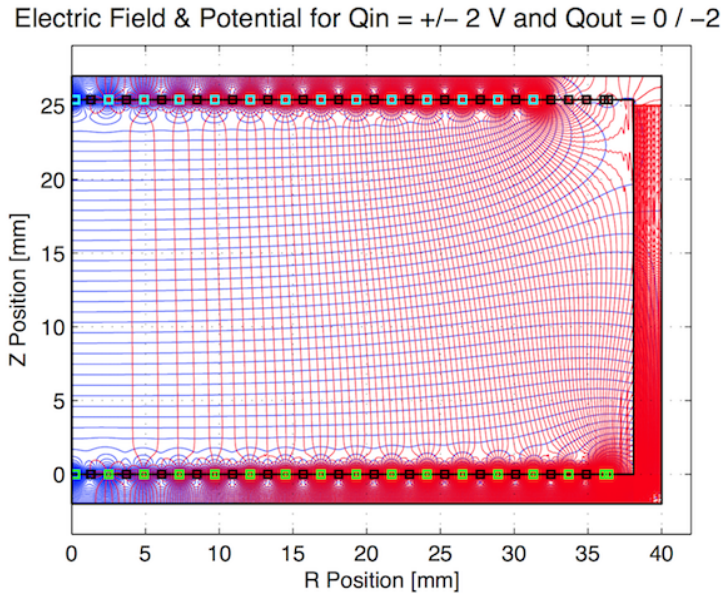

(b) E-field in defective detector.

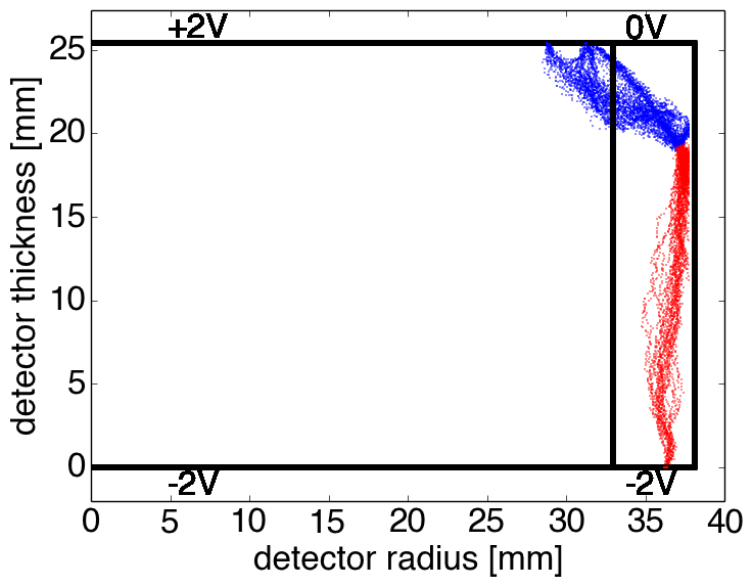

(d) Charge propagation in defective detector.

Figure 4.5: Simulated electric fields and charge propagation in an iZIP detector with a shorted outer charge channel (right) and without a short (left). Electric field and Monte Carlo plots provided by K. Schneck.[28] 


\section{Chapter 5}

\section{Charge Transport in Semiconductors}

\subsection{Band Structure and Bloch Wavefunctions}

Any understanding of the motion of charges through crystalline solids must begin from the perspective of wave mechanics. Crystals, by definition, are spatially periodic. According to Bloch's theorem, any eigenfunction of the wave equation for a particle in a periodic potential must have the form: $[48,49]$

$$
\psi_{k}(r)=u_{k}(r) e^{i k \cdot r}
$$

where $u_{k}(r)$ is a function with the same periodicity as the crystal lattice, and $k$ is a wavevector, called the crystal momentum ${ }^{1}$, which is a conserved quantity in most scattering processes, and which can take on any of a continuum of values within the Brillouin zone. For any given wavevector, $k$, there exists a discrete spectrum of energy eigenvalues, $\mathscr{E}_{n}(k)$, such that:

$$
\hat{H} \psi_{k}^{(n)}(r)=\mathscr{E}_{n}(k) \psi_{k}^{(n)}(r)
$$

\footnotetext{
${ }^{1}$ Here the wavevector is given its quantum-mechanical interpretation as representing a momentum, $p=\hbar k$.
} 


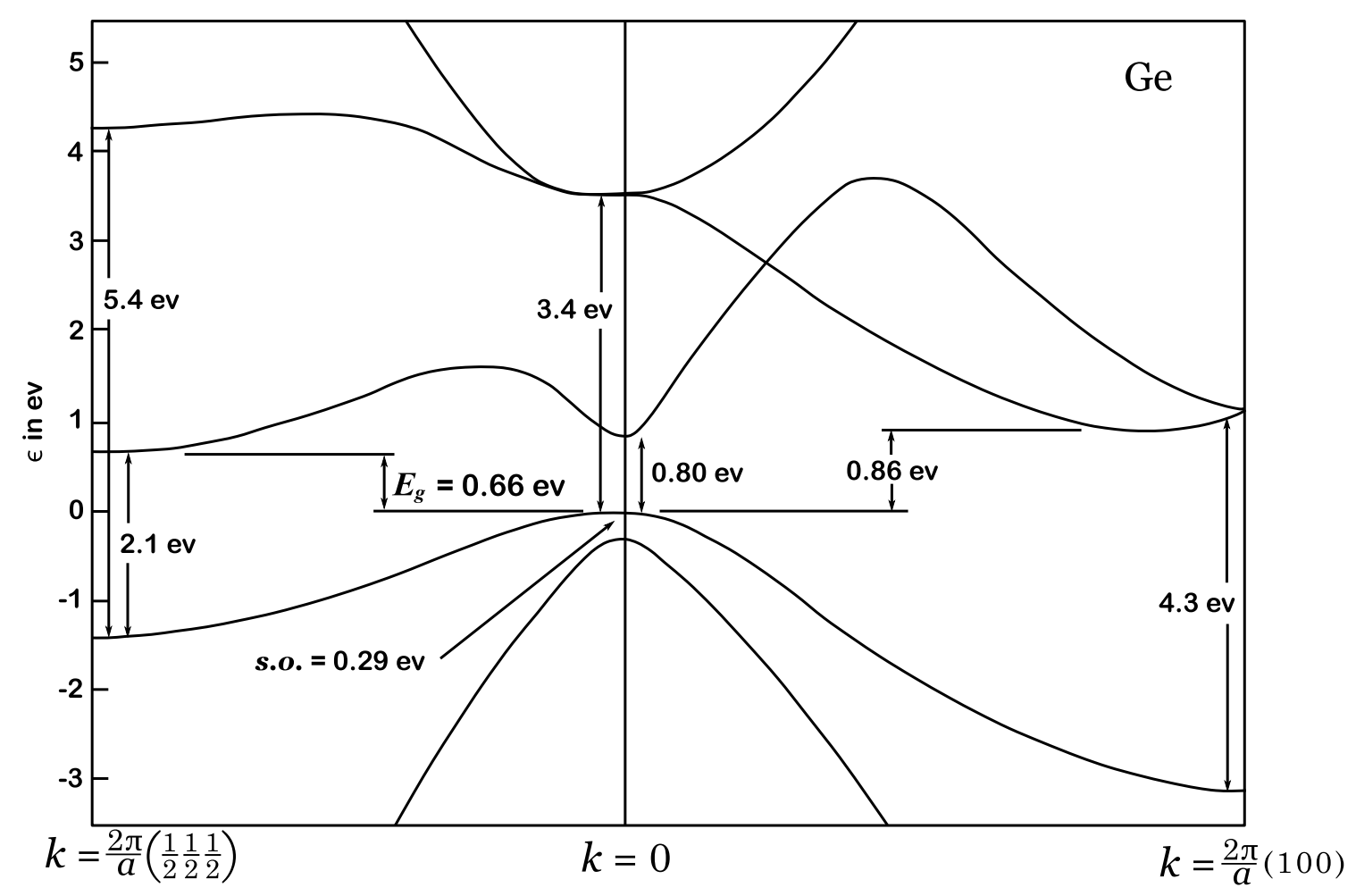

Figure 5.1: Structure of the valance and conduction bands of germanium along two crystal directions. (Adapted from [50].)

where, $n$, is an integer index. These discrete energy eigenvalues form a family of functions in momentum space. The set of all quantum eigenstates associated with a particular function is called an energy band. Figure 5.1 illustrates what some of these bands look like in germanium, projected along two directions in momentum space.

At a finite temperature, the thermally-averaged electron occupation number of any state, $\left\langle n_{i}\right\rangle$, is given by the Fermi-Dirac distribution:

$$
\left\langle n_{i}\right\rangle=\frac{1}{e^{\frac{\left.\mathscr{E}_{i}-\mu\right) /(k T)}{1}+1}}
$$

where, $\mu$, is the chemical potential. If $\mu$ falls within an energy band, the material is a metal. If $\mu$ falls in a gap between bands, the material is a semiconductor or insulator. Such is the case for germanium and silicon, which are semiconductors with band gap energies at $300 \mathrm{~K}$ of $0.66 \mathrm{eV}$ and $1.1 \mathrm{eV}$, respectively.[48, 49] The bands which play the 
largest role in charge transport are the valance and conduction bands, being defined as the bands with energy just below and just above $\mu$.

\section{$5.2 \quad$ Indirect-Gap Semiconductors}

If an electron in the valance band of a semiconductor absorbs enough energy, it is capable of making a quantum transition to an unoccupied state in the conduction band, leaving behind a newly-unoccupied state in the valance band, called a hole. Various relaxation processes will tend to reduce the energy of the system by driving the electrons and holes to their lowest energy states, causing them to collect in the local minima of their respective energy bands, also known as energy valleys. ${ }^{2}$

In germanium, the band gap is "indirect", meaning the lowest energy states for the electrons and holes do not align in momentum space. While the holes in the valance band assume their lowest energy at zero crystal momentum, the electrons in the conduction band reach a minimum of energy in the high momentum states at the very edge of the Brillouin zone in the $\langle 1,1,1\rangle$ crystal directions. [48] It is interesting to note that silicon, which has the same crystal structure as germanium, is also an indirect-gap semiconductor. However, in contrast to germanium, the energy minima in the silicon conduction band occur along the $\langle 1,0,0\rangle$ crystal directions. [48] These attributes of the conduction bands of silicon and germanium are illustrated in Figure 5.2 , which shows three-dimensional contours of constant energy in momentum space close to the energy valleys.

Indirect energy gaps in semiconductors can give rise to interesting optical properties, the most notable being that electrons and holes are incapable of annihilating through direct photon emission, as photons do not carry enough momentum to accommodate the large momentum difference between the two band minima. This is one of the reasons why light-emitting diodes are typically made from direct-gap semiconductors like gallium arsenide.

\footnotetext{
${ }^{2}$ Holes, being absences of electrons, reduce their energy by moving upward in the valance band.
} 


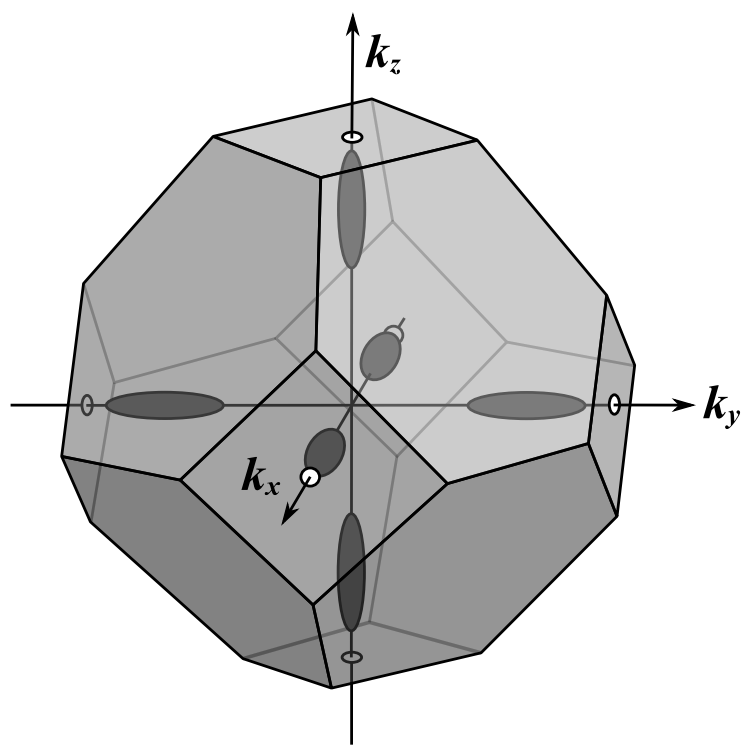

(a) Si Energy Valleys

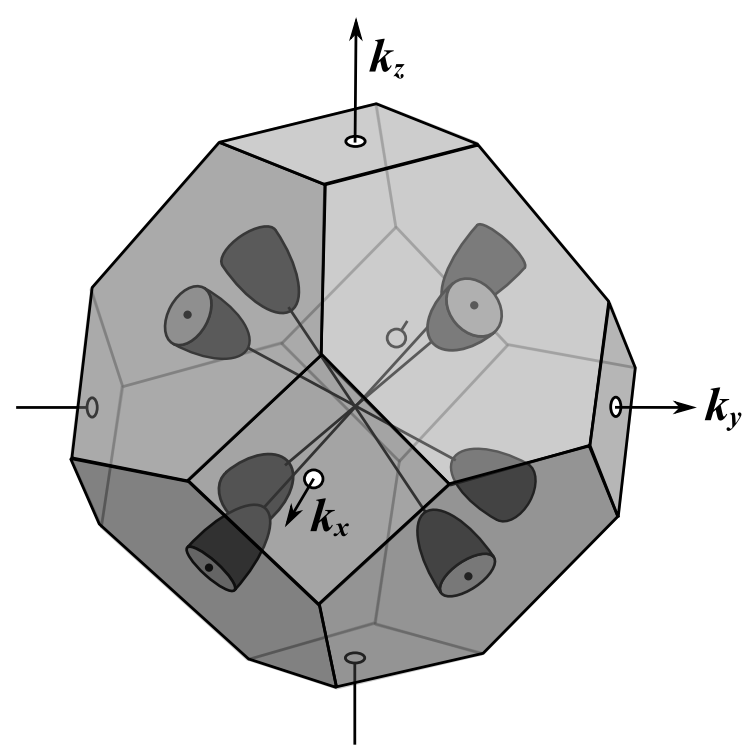

(b) Ge Energy Valleys

Figure 5.2: Three-dimensional plots of equal-energy surfaces in the conduction bands of silicon (a) and germanium (b). The shaded regions indicate the ellipsoidal equalenergy surfaces surrounding the energy minima near the edges of the Brillouin zone. In $\mathrm{Si}$, these minima occur along the six $\langle 100\rangle$ directions, while in Ge they occur along the eight $\langle 111\rangle$ directions. (Adapted from [51].)

\subsection{Semiclassical Model of Charge Transport}

On length scales large compared to the interatomic spacing, the Bloch wavefunctions described in $\S 5.1$ take on the appearance of plane waves. The energy vs. momentum functions for each band represent the dispersion relations for these waves, where the momentum, $p$, wavevector, $k$, energy, $\mathscr{E}$, and angular frequency, $\omega$, have the following quantum-mechanical relations:

$$
\begin{aligned}
\mathscr{E} & =\hbar \omega \\
p & =\hbar k
\end{aligned}
$$


Because the densities of the free electrons and holes in undoped semiconductors are low when the thermal energy is substantially lower than the band gap, the wavefunctions of the free charge carriers may be approximated as localized, non-overlapping wavepackets. These wavepackets travel with group velocities, $v_{g}$, given by:

$$
v_{g}=\partial_{k} \omega=\partial_{p} \mathscr{E}
$$

To a good approximation, the mean position, $q$, and momentum, $p$, of each wavepacket can be described by the classical Hamiltonian system described by equations 5.6, where the function, $\mathscr{E}(p)$ is the energy vs. momentum function for the energy band that the charge carrier is occupying, and $V(q)$ is the electrostatic potential energy of the charge carrier due to the presence of an external field. ${ }^{3}$

$$
\begin{aligned}
\mathscr{H} & =V(q)+\mathscr{E}(p) \\
\dot{p} & =-\partial_{q} \mathscr{H}(q, p)=-\partial_{q} V(q)=F \\
\dot{q} & =\partial_{p} \mathscr{H}(q, p)=\partial_{p} \mathscr{E}(p)=v_{g}
\end{aligned}
$$

As mentioned in $\S 5.2$, various relaxation processes will tend to reduce the kinetic energies of the free charge carriers until they collect into local minima of energy in momentum space. In the conditions relevant to our experiment, the level of thermal excitations is low enough ${ }^{4}$ that the charge carriers all remain very close to their energy minima. Consequently, the energy vs. momentum relations for the charge carriers may be well approximated by a Taylor expansion truncated at the quadratic term. ${ }^{5}$ A general quadratic energy vs. momentum relation may be written as:

$$
\mathscr{E}(p)=\frac{1}{2}\left(p-p_{0}\right) \cdot \underline{m}^{-1} \cdot\left(p-p_{0}\right)+\mathscr{E}_{0}
$$

\footnotetext{
${ }^{3}$ Note that the effects of a magnetic field may be incorporated into this model by substituting $p-e A$ for $p$ in the Hamiltonian, where $A$ is the magnetic vector potential, and $e$ is the electric charge of the charge carrier.

${ }^{4}$ Thermal energy at $1 \mathrm{~K}=8.6 \cdot 10^{-4} \mathrm{eV}$

${ }^{5}$ Note that this argument applies only approximately to the holes, as their energy vs. momentum function is not smooth at $k=0$. The second and all higher derivatives do not exist.
} 
where $p_{0}$ is the momentum vector of the local minimum. The mass tensor, $\underline{m}$, is a general, real-valued, symmetric, positive-definite, $3 \times 3$ matrix. If we insert this energy vs. momentum relation into the equations of Hamiltonian mechanics, we obtain a modified version of Newton's Second Law:

$$
\begin{aligned}
\dot{q} & =\partial_{p} \mathscr{E}(p)=\underline{m}^{-1} \cdot\left(p-p_{0}\right) \\
\ddot{q} & =\underline{m}^{-1} \cdot \dot{p}=\underline{m}^{-1} \cdot F \\
F & =\underline{m} \cdot \ddot{q}
\end{aligned}
$$

Therefore, we see that the applied force, $F$, and the acceleration vector, $\ddot{q}$, are not parallel to each other unless $F$ lies within a subspace of spanned by degenerate eigenvectors of $\underline{m}$. Such alignment will be rare if $\underline{m}$ has multiple different eigenvalues, which is the case for the mass tensors of the energy valleys in the conduction bands of germanium and silicon.

\begin{tabular}{|c|c|c|}
\hline & Silicon & Germanium \\
\hline$m_{h} / m_{0}$ & 0.5 & 0.35 \\
\hline$m_{e||} / m_{0}$ & 0.91 & 1.58 \\
\hline$m_{e \perp} / m_{0}$ & 0.19 & 0.081 \\
\hline
\end{tabular}

Table 5.1: Hole and electron masses in $\mathrm{Si}$ and Ge relative to the free-space electron mass, $m_{0} \cdot[44]$

This quadratic approximation is valid only in the vicinity of one particular energy valley. In silicon, there are six distinct energy valleys, and in germanium there are four. ${ }^{6}$ The mass tensors associated with these valleys are all different, although they are constrained by crystal symmetries to share the same set of eigenvalues.

In general, the eigenvectors of the mass tensor for each energy valley lie either parallel or perpendicular to the momentum vector, $p_{0}$, which defines the location

\footnotetext{
${ }^{6}$ Because the energy valleys of germanium occur at the edges of the Brillouin zone, and because opposite sides of the Brillouin zones are identified, energy minima lying in opposite directions from the origin represent the same point in momentum space. This means that there are only four unique valleys rather than the eight which would be expected from a set of valleys lying in the eight $\langle 1,1,1\rangle$ directions.
} 
of the local energy minimum, with the two perpendicular eigenvectors forming a degenerate subspace. Table 5.1 gives the parallel, $m_{e \|}$, and perpendicular, $m_{e \perp}$, eigenvalues of the electron mass tensors in germanium and silicon, relative to the mass of the electron in vacuo, $m_{0}$.

The holes, which have a single energy minimum at zero momentum, are well approximated by an isotropic quadratic energy surface centered at zero with the relative hole masses, $m_{h}$, given in the top row of Table 5.1 .

\subsection{Scattering Processes}

One interesting consequence of Bloch's theorem is that a crystal presents no impediment to the motion of free charge carriers as long as it is perfectly periodic. ${ }^{7}$ Therefore, a free carrier will only scatter to a new momentum state if it experiences some sort of interruption in the otherwise perfect periodicity of the crystal lattice. Such non-periodic perturbations can be created by crystal defects, impurities, lattice vibrations, or the presence of other free carriers.

Because crystal defects and impurities break the discrete translational symmetry of the crystal lattice, they allow for scattering processes in which the momentum of free carriers is not conserved. However, defects and impurities do not break the timetranslation symmetry of the crystal lattice, and therefore the energy of free carriers is preserved in these scattering processes.

Lattice vibrations, being non-equilibrium distortions of the crystal lattice, are likewise capable of scattering free carriers. However, the scattering processes involving lattice vibrations have two important differences from impurity scattering:

First, unlike static defects or impurities, lattice vibrations necessarily involve a temporal variation in the structure of the crystal lattice, which explicitly breaks time-translation symmetry. It is therefore possible for not only momentum, but also energy to be exchanged between the free carriers and the lattice vibrations.

The second distinction between scattering from impurities and lattice vibrations

\footnotetext{
${ }^{7}$ Bloch was partly motivated to develop his theory by measurements which showed that free charges could posses scattering lengths far in excess of the interatomic spacing.[52]
} 
is that the amplitudes of lattice vibrations are temperature-dependent. To properly describe this temperature dependence at the energy scales relevant to our experiment, the quantum nature of the lattice vibrations must be taken into account. The thermally-averaged number of sound quanta, or phonons, occupying a particular vibrational mode with frequency $\omega_{i}$ is given by the Bose-Einstein distribution:

$$
\left\langle n_{i}\right\rangle=\frac{1}{e^{\hbar \omega_{i} /(k T)}-1}
$$

At any non-zero temperature, the crystal will contain a thermal bath of phonons from which free carriers will scatter. Because the phonon density depends on temperature, so does the mean-free-path for phonon scattering, unlike the mean-free-path for impurity or defect scattering.

The quantum nature of phonon interactions leads also to another important consequence. The classical process of a carrier scattering from a crystal vibration may be represented quantum-mechanically as the absorption of a phonon by the free carrier. However, the fact that the quantum Hamiltonian is Hermetian requires that the inverse process, that of phonon emission by a free carrier, must also be allowed. This implies that phonons need not be present in the initial quantum state of the system in order for phonon scattering to take place. (See $\S 5.5$ and §5.6.)

In the conditions relevant to the experiment described in this dissertation, scattering between carriers is only important during a short interval after the initial energy deposition event when the density of free carriers is high. In our simulations, the interaction between charge carriers is modeled as a pairwise Coulomb force. Further detail is provided in Chapter 9.

Figure 5.3 illustrates the two primary effects of these scattering processes: "intravalley" scattering, which leaves the charge carrier in the same energy valley as its initial state, and "inter-valley" scattering, which causes the carrier to transition to a momentum state lying in a different energy valley. Inter-valley scattering necessarily requires a large change in crystal momentum, but could potentially involve no change in energy. Intra-valley scattering requires a much smaller change in crystal momentum, and therefore occurs much more easily and frequently. 


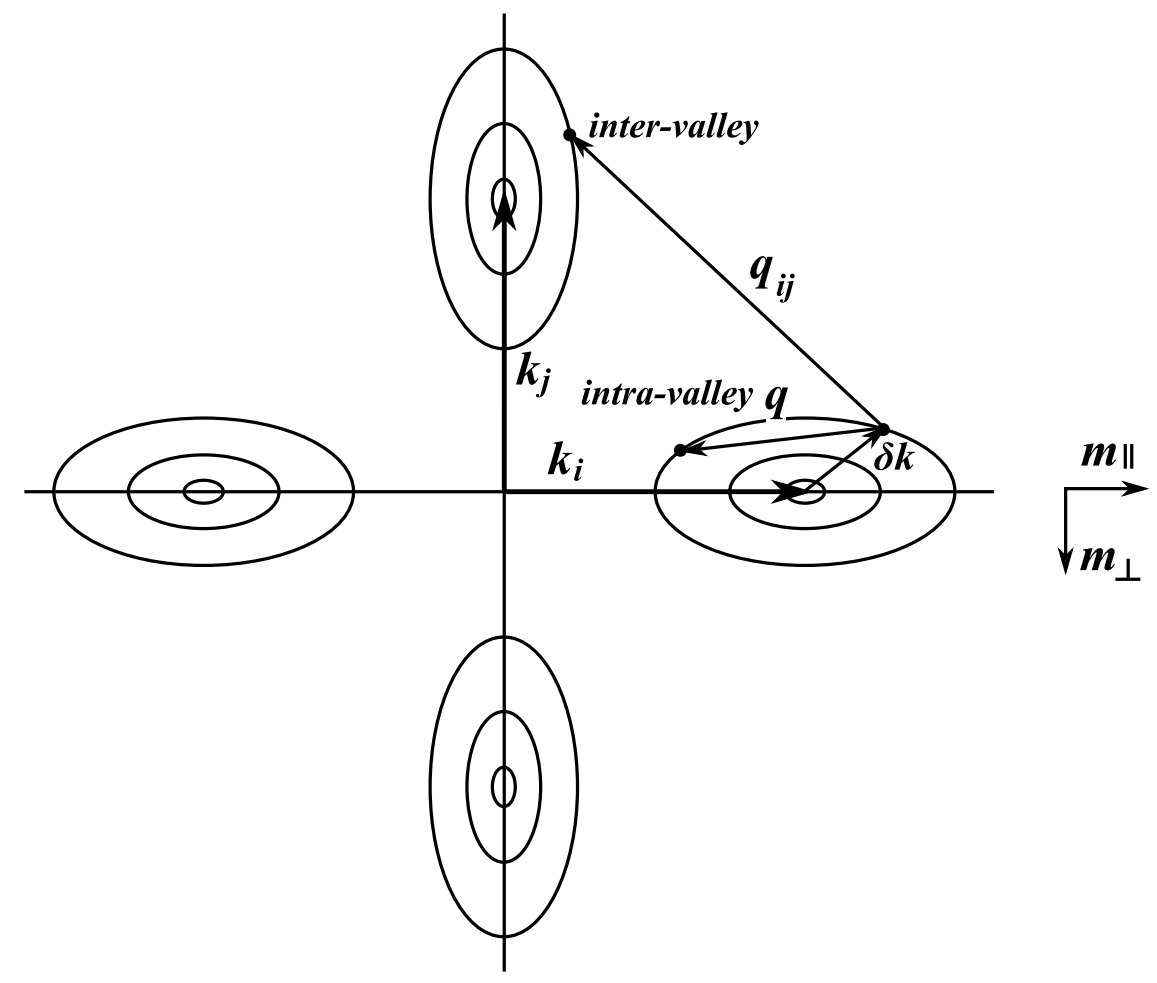

Figure 5.3: Diagram in momentum space showing scattering of electrons both within an energy valley (intra-valley scattering), and between energy valleys (inter-valley scattering). (Adapted from [53].)

\subsection{Electron-Phonon Interaction}

As discussed in the previous section, free electrons and holes in semiconductors can interact with the crystal lattice through the emission or absorption of a phonon. This interaction is mediated through a quantum interaction Hamiltonian of the form:

$$
\mathscr{H}_{I}=\int d^{3} x \underline{X} \cdot \underline{s} \psi^{*} \psi
$$

where $\underline{s}$ is the crystal strain tensor, $\underline{X}$, is the deformation potential tensor, and $\psi$ is the electron field. This interaction conserves energy and momentum, and it allows an electron or hole to change momentum by creating or destroying a phonon. 
The total interaction rate can be calculated by integrating the square of the transition amplitude over the phonon phase space. The transition amplitudes and interaction rates are derived in $\S$ F.12 using a continuum approximation for the electron and phonon fields. Analytic forms are found for the rates in the special case of an isotropic material.

To incorporate this scattering process into our semiclassical model, we introduce classical Poisson processes for phonon emission and absorption into our Monte Carlo simulation of charge transport with average rates given by the quantum mechanical transition rates. [4]

Unfortunately, the integrals F.92 and F.93 only have closed-form solutions in the isotropic case. In Kyle Sundqvist's Monte Carlo simulation, these integrals are computed numerically for each charge carrier as a function of that charge carrier's velocity.[4]

In order to increase the speed of the simulation, we decided to employ the HerringVogt approximation, in which the momentum space is stretched so that the surfaces of constant energy become spheres.[54] The variables in the transformed space, denoted by “*”, are defined by the relations:[44]

$$
\begin{aligned}
k & =m_{c}^{-1 / 2} \underline{m}^{1 / 2} \cdot k^{*} \\
E & =m_{c}^{-1 / 2} \underline{m}^{1 / 2} \cdot E^{*} \\
x & =m_{c}^{1 / 2} \underline{m}^{-1 / 2} \cdot x^{*} \\
v & =m_{c}^{1 / 2} \underline{m}^{-1 / 2} \cdot v^{*} \\
\mathscr{E} & =\mathscr{E}^{*} \\
\omega & =\omega^{*} \\
m_{c} & \equiv \frac{3}{\frac{1}{m_{e \|}}+\frac{1}{m_{e \perp}}}
\end{aligned}
$$

where the wavevector, $k$, is measured from the center of the valley. We see that the equal-energy surfaces are spheres in the new momentum space:

$$
\mathscr{E}^{*}=\mathscr{E}=\frac{1}{2} k \cdot \underline{m}^{-1} \cdot k=\frac{1}{2} m_{c}^{-1} k^{*} \cdot \underline{m}^{1 / 2} \underline{m}^{-1} \underline{m}^{1 / 2} \cdot k^{*}=\frac{1}{2} m_{c}^{-1} k^{*} \cdot k^{*}
$$


The purpose of this transformation is to reformulate the problem so that it can be approximated as closely as possible by an isotropic scattering process. The reformulation could be made exact if not for the fact that the speed of sound in the transformed space is no longer isotropic. We approximate the integral using an isotropic speed of sound in the transformed space, which gives a closed-form solution while simultaneously introducing a certain irreducible error. The values of the deformation potential and speed of sound used in the Herring-Vogt approximation are given in Table 5.2.

This approximation, utilized by Peter Redl in his Monte Carlo simulation, increases the simulation speed by a factor of 20 relative to the Sundqvist simulation. However, the price paid for this gain in computational speed is the explicit violation of momentum conservation in the phonon scattering process. The scale and relative importance of the errors introduced into the simulation as a consequence of this approximation are discussed in detail in Chapter 9.

\begin{tabular}{|c|c|c|c|c|}
\hline & \multicolumn{2}{|c|}{ Silicon } & \multicolumn{2}{c|}{ Germanium } \\
\hline & Electrons & Holes & Electrons & Holes \\
\hline$X(\mathrm{eV})$ & 9.0 & 5.0 & 11.0 & 3.4 \\
\hline$c_{s}(\mathrm{~km} / \mathrm{s})$ & \multicolumn{2}{|c|}{9.0} & \multicolumn{2}{c|}{5.4} \\
\hline
\end{tabular}

Table 5.2: Values used in the Herring-Vogt approximation for the deformation potential, $X$, and the longitudinal speed of sound, $c_{s} \cdot[44]$

\subsection{Carrier Mobility}

One of the interesting consequences of energy and momentum conservation in phonon interactions is that charge carriers cannot emit phonons if their initial speed is less than the speed of sound. Figure 5.4 provides a simple graphical proof of this effect in one dimension. The blue curve represents the quadratic energy vs. momentum relation for an electron or hole. The green cones represent the set of all possible final states after the emission of a single phonon, starting from the black dot, assuming conservation of energy and momentum. Because the final state of the electron or hole is kinematically constrained to lie on the blue curve, the intersections between 


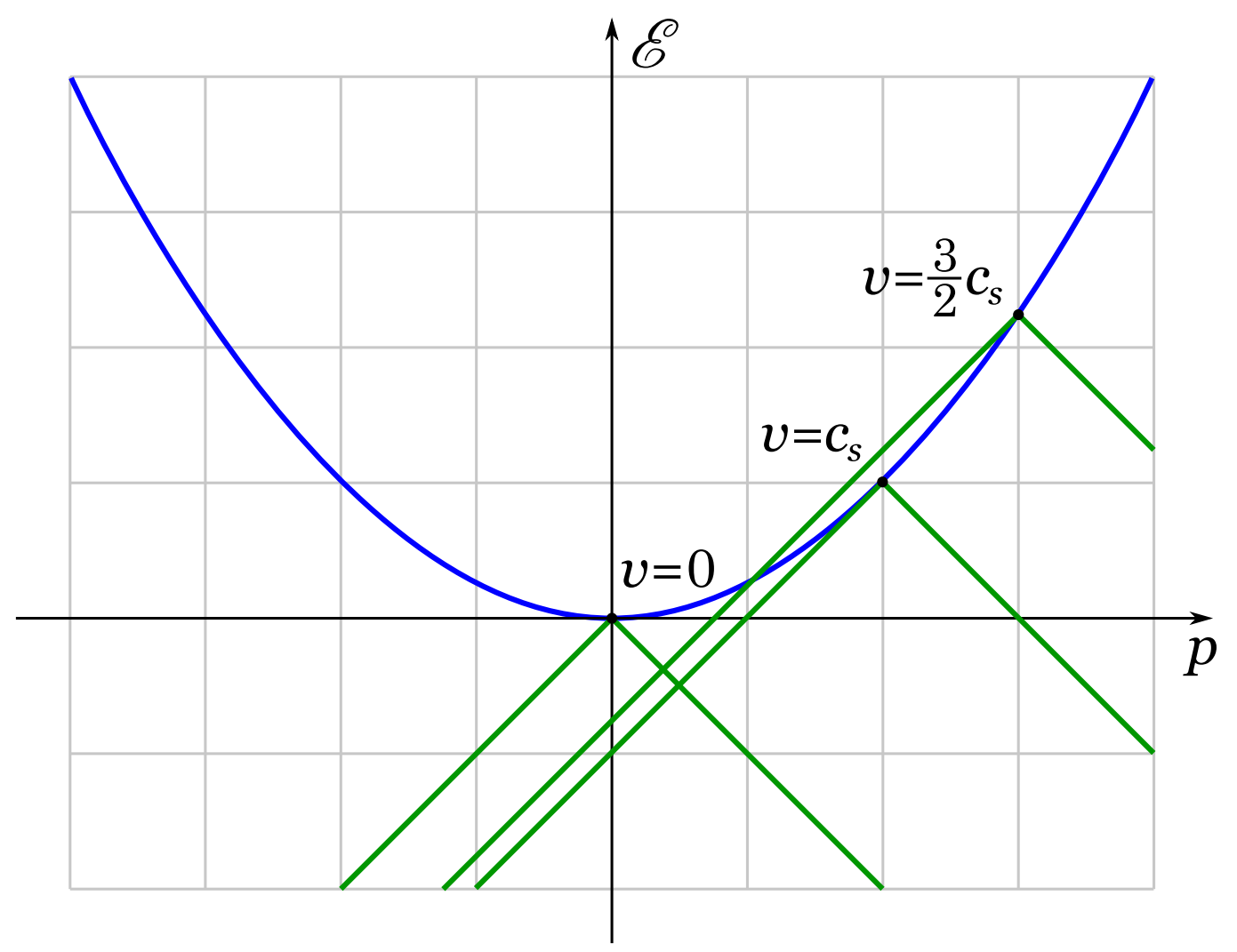

Figure 5.4: A simple graphical illustration of the kinematics of phonon emission in one dimension.

the green cones and the blue curve represent allowed final states. Note that phonon emission is only possible from starting points on the blue curve with slope greater than the speed of sound, $c_{s}$. The carrier velocity, $v$, which is equal to the slope of the blue curve, is indicated for each of the three starting points.

As the crystal temperature approaches zero, the probability of phonon absorption approaches zero, and phonon scattering becomes dominated by the process of phonon emission. Therefore, in ultra-pure crystals where impurity scattering is negligible, charge carriers accelerate in a uniform field without resistance until they exceed the velocity of sound, at which point they begin to loose energy through phonon emission. 


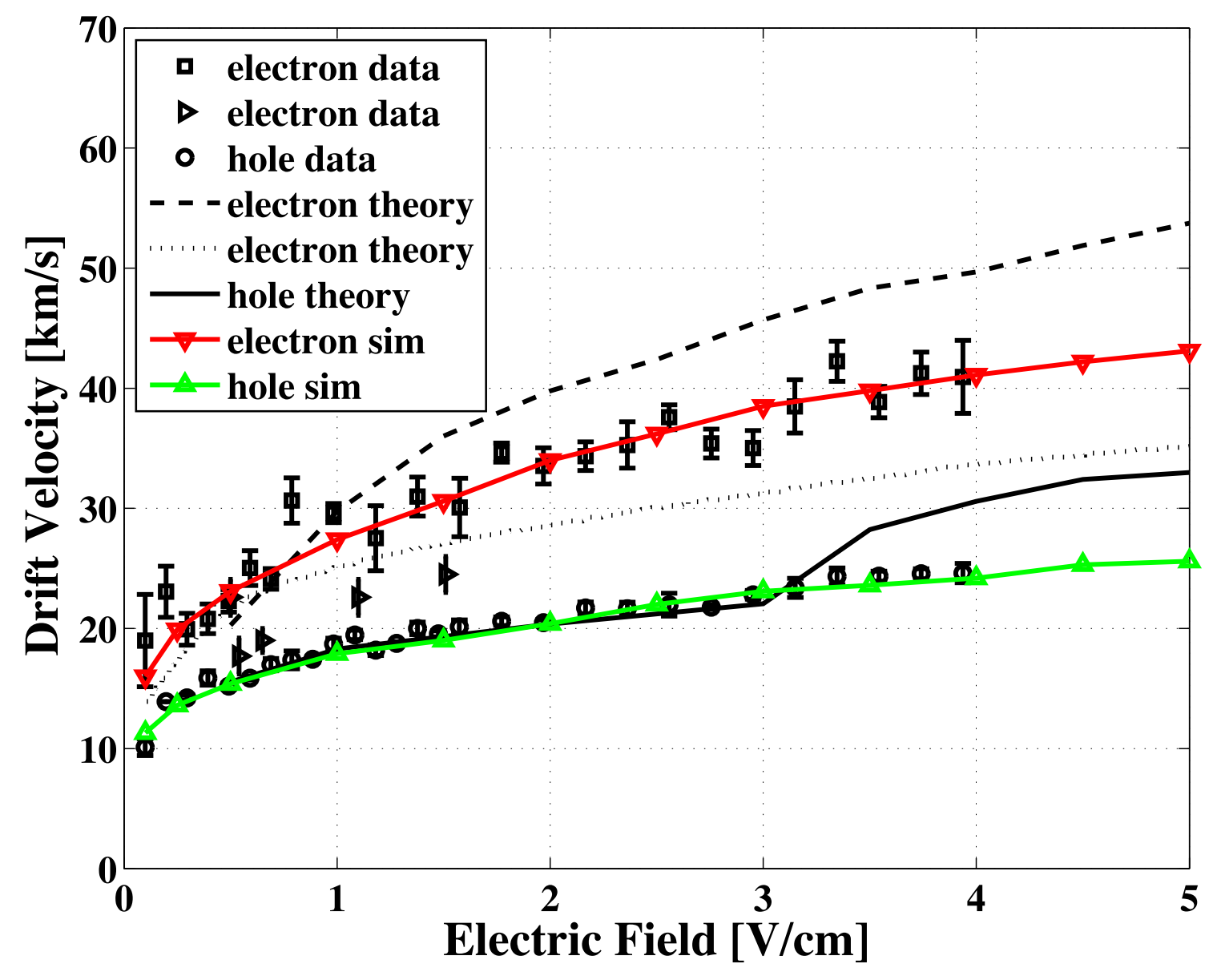

Figure 5.5: Comparison of simulated and measured carrier drift velocity as a function of electric field along the $[1,0,0]$ axis for ultra-pure $\left(N_{a}=1.83 \times 10^{11} \mathrm{~cm}^{-3}\right) \mathrm{Ge}$ crystals at $31 \mathrm{mK} .[44,55]$

Consequently, charge carriers will tend to travel with a drift velocity comparable to the speed of sound over a wide range of applied electric field strengths, resulting in a non-Ohmic conductivity. Both experiment and simulation show a power-law relation between carrier drift velocity and electric field of the form $v_{d} \propto E^{1 / 5}$.[44] Figure 5.5 shows the comparison between our experimental data and Monte Carlo simulations. 


\subsection{Electron Anisotropy}

As discussed in $\S 5.3$, electrons in silicon and germanium occupy energy valleys with anisotropic mass tensors. An electron occupying a particular energy valley with mass tensor, $\underline{m}$, accelerates in the presence of a uniform electric field, $E$, with a magnitude and direction given by $-e \underline{m}^{-1} \cdot E$. The electron continues to gain velocity in this direction until a scattering event occurs, mediated either by a phonon interaction or by a crystal defect or impurity.

Because interactions involving low momentum transfer are more likely than interactions involving high momentum transfer, most scattering events cause the electron to transition to a new state within the same energy valley. After the scattering event, the electron begins to accelerate again in the same direction as before. The result is a random walk plus a mean drift in the direction of the acceleration, $-e \underline{m}^{-1} \cdot E$.

Occasionally, a scattering event will cause a momentum transfer of sufficient magnitude to mediate a quantum transition to a new energy valley. After such an intervalley scattering event takes place, the electron begins to accelerate in a new direction determined by the mass tensor of the new energy valley.

Inter-valley scattering events occur with a mean rate, $\Gamma_{\mathrm{IV}}$, which is a function of the impurity and defect concentration, the crystal temperature, and the electric field strength. Under the assumption that the scattering events may be well modeled as a Poisson process, the probability, $p(t)$ that an electron remains in the same valley after time, $t$, is given by the exponential distribution:

$$
p(t)=e^{-\Gamma_{\mathrm{IV}} t}
$$

During the time, $t$, the electron drifts a distance, $x=-v_{d} \cdot \hat{E} t$ projected onto the direction of the applied electric force, $-\hat{E}$. Therefore, the probability, $p(x)$, that an electron drifts for a distance, $x$, while remaining in the same energy valley is given by a similar exponential law:

$$
p(x)=e^{-x / \lambda_{\mathrm{IV}}}
$$

where $\lambda_{\mathrm{IV}} \equiv-v_{d} \cdot \hat{E} / \Gamma_{\mathrm{IV}}$ is defined to be the projection of the mean inter-valley 
scattering length onto the direction of the applied force.

Because the inter-valley scattering rate, $\Gamma_{\mathrm{IV}}$, is the sum of the rates of all intervalley scattering processes, the following relations will hold between the total scattering rate and length, and those of the constituent processes:

$$
\begin{aligned}
\Gamma_{\mathrm{IV}} & =\Gamma_{\mathrm{IV}}^{(\mathrm{C})}+\Gamma_{\mathrm{IV}}^{(\mathrm{A})}+\Gamma_{\mathrm{IV}}^{(\mathrm{E})} \\
\frac{1}{\lambda_{\mathrm{IV}}} & =\frac{1}{\lambda_{\mathrm{IV}}^{(\mathrm{C})}}+\frac{1}{\lambda_{\mathrm{IV}}^{(\mathrm{A})}}+\frac{1}{\lambda_{\mathrm{IV}}^{(\mathrm{E})}}
\end{aligned}
$$

where the superscripts represent the scattering processes mediated by the crystal defects and impurities (C), by phonon absorption (A), and by phonon emission (E).

The division of the scattering rate into a sum of contributions from these three constituent processes is useful not only as a means of understanding their relative importance to the overall rate, but it also clarifies the functional dependence of the scattering rate on the impurity and defect concentration, the crystal temperature, and the electric field strength, as each rate associated with a particular constituent processes is primarily a function of only one of these parameters.

The first term in Equation 5.15, $\Gamma_{\mathrm{IV}}^{(\mathrm{C})}$, is determined solely by the concentration of crystal defects and impurities and by the scattering cross-section which they present to free carriers. In the germanium crystals used in the SuperCDMS experiment, we measure the mean-free-path for inter-valley scattering induced by impurities and defects to be on the order of $20 \mathrm{~cm}$.

The second term, $\Gamma_{\mathrm{IV}}^{(\mathrm{A})}$, which depends only on temperature. is determined by the thermal phonon background, and is therefore proportional to the thermal occupation number of the phonon modes which are responsible for inter-valley transitions. Because this number is exponentially suppressed at low temperatures, this process very quickly becomes negligible relative to impurity and defect scattering in SuperCDMS crystals at temperatures below $4 \mathrm{~K}$.

The third term, $\Gamma_{\mathrm{IV}}^{(\mathrm{E})}$, depends only on the applied electric field strength. Electrons need a minimum energy to undergo an inter-valley transition by phonon emission. Therefore, this rate is determined by the number of electrons in the high-energy tail of the energy distribution of the free electrons in the presence of an electric field. The 
population of electrons capable of emitting inter-valley phonons is larger in stronger fields because the probability is higher that an electron can reach a high velocity, and consequently a high kinetic energy, before undergoing a scattering event. This process is negligible relative to impurity and defect scattering in the SuperCDMS crystals for electric fields smaller than $1 \mathrm{~V} / \mathrm{cm}$.

The combination of defect and impurity concentration, crystal temperature, and electric field strength together determine the overall inter-valley scattering rate, $\Gamma_{\mathrm{IV}}$, and the corresponding inter-valley scattering length, $\lambda_{\mathrm{IV}}$. Based on this length, three regimes of carrier transport may be distinguished by their qualitatively-different transport properties.

In the anisotropic regime, the inter-valley scattering length is much larger than the size of the crystal sample. In this case, electrons propagate across the entire thickness of the crystal without transitioning to a different valley. The initial distribution of electrons among the different energy valleys is therefore maintained throughout the transport process, with the drift velocity of each electron depending on the valley the electron is currently occupying.

This type of transport is illustrated by the Monte Carlo simulation plotted in Figure 5.6. The simulation shows the paths of electrons in a germanium crystal with a uniform electric field of strength $1 \mathrm{~V} / \mathrm{cm}$. The electrons are all given an initial position at the origin of the coordinate system, with equal populations of electrons occupying the four different energy valleys. The most notable aspect of the transport process is how the different mass tensors of the four valleys cause a spatial separation to develop between the four different populations.

In the isotropic regime, the inter-valley scattering length is much smaller than the size of the crystal. In this case, electrons cannot cross the thickness of the crystal while remaining in the same energy valley. Instead, electrons scatter frequently between the different energy valleys, spending roughly equal time in each. The average drift velocity will then be the average of the drift velocities of all of the energy valleys, which is isotropic. In this regime, the electron transport becomes very similar to that of the holes when viewed on macroscopic scales. 
The third regime is intermediate between the isotropic and anisotropic cases, and occurs when the inter-valley scattering length is comparable to the size of the crystal. In this case, carriers are capable of traversing the entire crystal thickness while remaining in the same energy valley, but also have a non-negligible probability of undergoing one or more inter-valley transitions during the transport process. This regime of carrier transport is of most relevance to the experiment described in this dissertation.

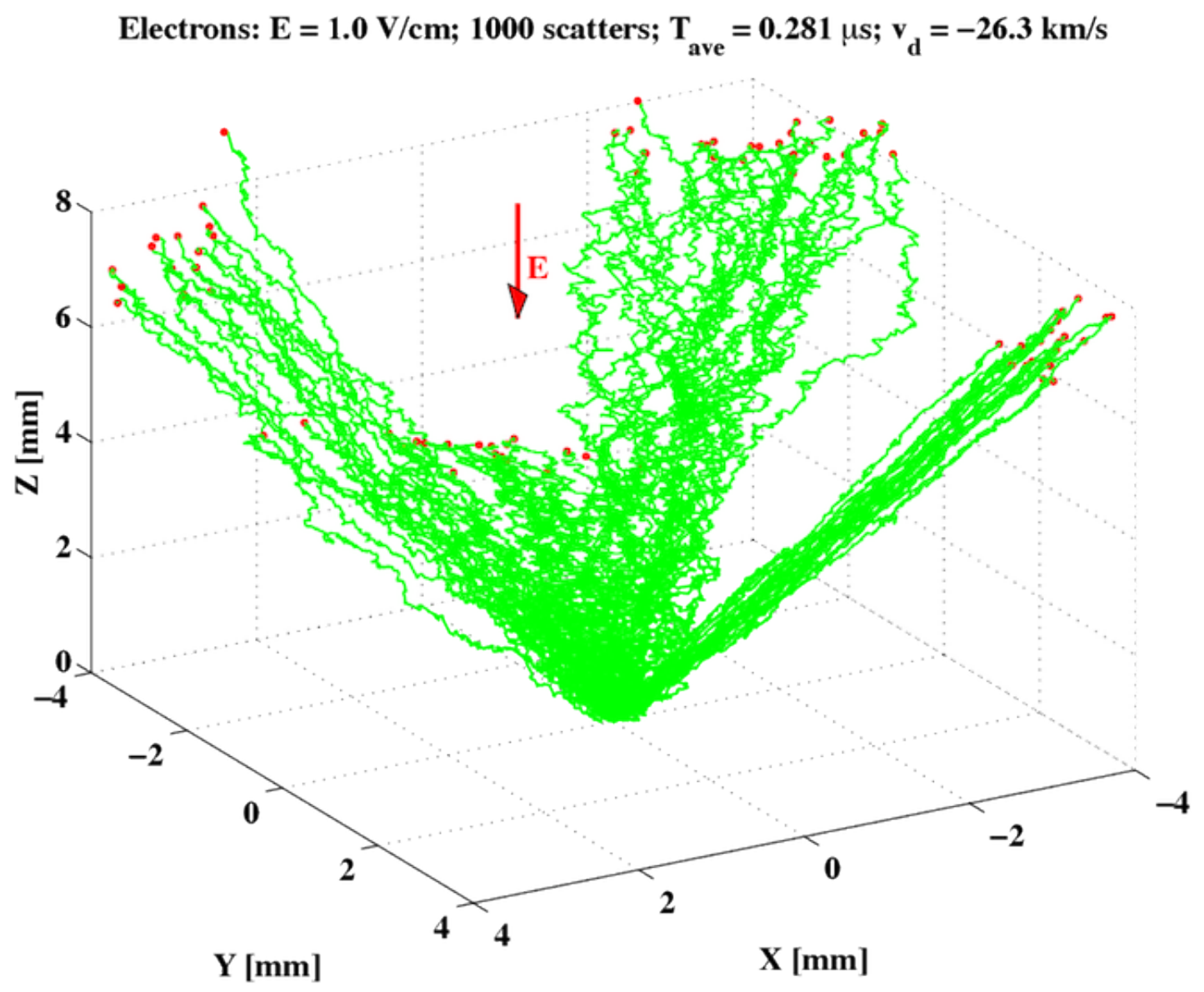

Figure 5.6: Monte Carlo simulation of electron propagation in germanium in a uniform electric field, starting from an initial position at the origin of the coordinate system. Note how the electrons separate into four clusters.[44] 


\section{Chapter 6}

\section{Experimental Setup}

\subsection{Overview}

The system described in this chapter was designed to directly measure the propagation of charges in germanium by forming a two-dimensional image of charge density on the surface of a small germanium test crystal.

The charge carriers are excited on one face of a germanium crystal by a focused laser pulse. Once free, they are drifted through the crystal by a uniform electric field, and collected by electrodes on the opposite face. A two-dimensional map of the charge density is formed using an electrically controllable mirror which can scan the excitation point across the illuminated face of the crystal.

\subsection{Crystal Sample}

The crystal under test was cut from a $3.89 \mathrm{~mm}$ thick wafer of high purity germanium. ${ }^{1}[56]$ The front and back faces are $1 \mathrm{~cm} \times 1 \mathrm{~cm}$, and lie in the $(0,0,1)$ crystal plane, while the sides lie in the $(1,1,0)$ and $(1, \overline{1}, 0)$ planes. The illuminated (front) face of the crystal is patterned with an aluminum-tungsten mesh electrode, with $10 \mu \mathrm{m}$

\footnotetext{
${ }^{1} \mathrm{~N}$-type, net impurity concentration $\approx 8 \mathrm{E} 10 \mathrm{~cm}^{-3}$, etch pit dislocation density $\approx 3.2 \mathrm{E} 3 \mathrm{~cm}^{-2}$, mobility at room temperature $\approx 2.7 \mathrm{E} 4 \mathrm{~cm}^{2} /$ Vs. (From Umicore)
} 
pitch and $20 \%$ coverage. $^{2}$ The non-illuminated (back) face is covered by two solid electrodes, one of which is circular with a diameter of $160 \mu \mathrm{m}$, and separated by a $10 \mu \mathrm{m}$ gap from the other electrode, which covers the rest of the face of the crystal. Both electrodes on the non-illuminated face are connected to ground through $10.75 \mathrm{M} \Omega$ of resistance.

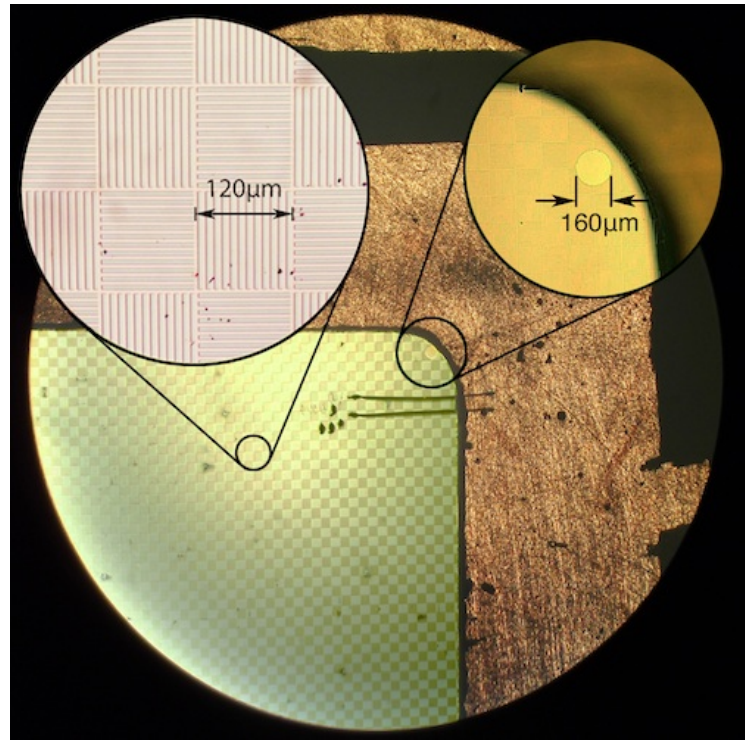

(a) Illuminated surface.

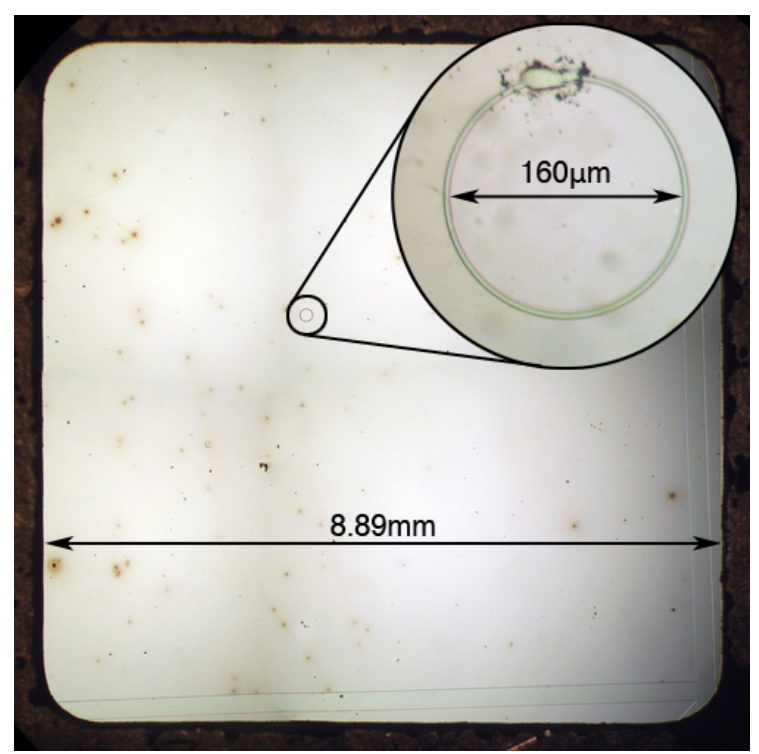

(b) Non-illuminated surface.

Figure 6.1: Microscope images of the surfaces of the germanium crystal sample. Annotations show the sizes of various features.

\subsection{Optical System}

Figure 6.2 shows the experimental payload with annotations indicating the various components and their nominal operational temperatures. A benchtop photograph of the crystal sample is shown in Fig. 6.3b. The sample is mounted to a copper baseplate (bottom of photograph), and is cooled to $630 \mathrm{mK}$ under vacuum in a He-3

\footnotetext{
Si.

${ }^{2}$ Electrodes composed of tri-layer consisting of $40 \mathrm{~nm}$ of $\mathrm{W}$ on $20 \mathrm{~nm}$ of $\mathrm{Al}$ on $40 \mathrm{~nm}$ of amorphous
} 


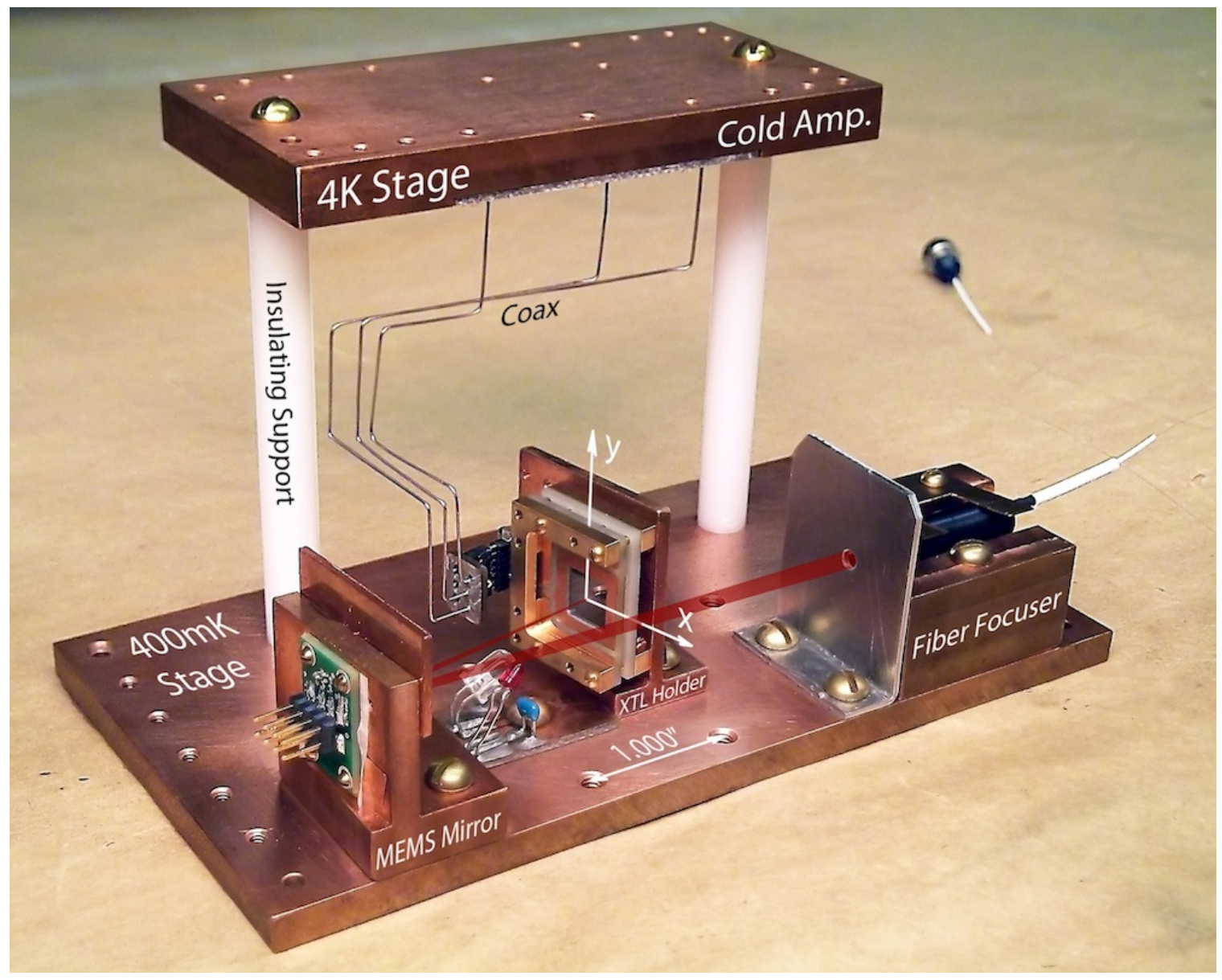

Figure 6.2: Annotated photograph of experimental payload on benchtop with infrared shield removed.

cryostat. The crystal sample is shielded from both electrical noise and $4 \mathrm{~K}$ thermal radiation by a copper enclosure mounted on the baseplate.

To generate free charge carriers, the illuminated face of the test crystal is exposed to a $20 \mu \mathrm{W}, 100 \mathrm{~ns}$ pulse of $650 \mathrm{~nm}$ laser light, focused to a $70 \mu \mathrm{m}$ diameter spot, which creates a cloud of electron-hole pairs near the illuminated surface. ${ }^{3}$ Carriers are propagated through the bulk of the crystal in the uniform electric field induced

\footnotetext{
${ }^{3}$ The short pulses of laser light are generated by a Berkeley Nucleonics 6040 pulsed laser source, and are coupled into the ${ }^{3} \mathrm{He}$ cryostat through a single-mode optical fiber. The peak laser power output from the BNC 6040 is set to $2 \mathrm{~mW}$. The laser light passed through a $20 \mathrm{~dB}$ optical attenuator at room temperature to reduce the peak laser power to $20 \mu \mathrm{W}$.
} 
by the DC bias voltage of the mesh electrode on the illuminated face. The sign of the bias voltage determines whether electrons or holes propagate through the bulk of the crystal. Once collected by the electrodes on the non-illuminated face, the charge carriers produce proportional pulses in voltage, which are measured by highimpedance MESFET amplifiers, mounted on the 4K stage in the He-3 cryostat.[57, 58]

The two-dimensional pattern of charge density is determined by measuring the charge collected by the small, circular electrode as a function of the position of the excitation point on the illuminated face. Because translation of the excitation point causes a corresponding translation of the charge density pattern, this procedure is equivalent to keeping the excitation point fixed, while moving the small, circular electrode to probe different regions of the charge density pattern. The position of the excitation point is controlled by means of a Micro Electro-Mechanical System (MEMS) mirror from Mirrorcle Technologies, Inc.[59] ${ }^{4}$ The MEMS mirror can be tilted along two axes by computer control, and was modified by the vendor for operation below 1K. (See Figure 6.4.)

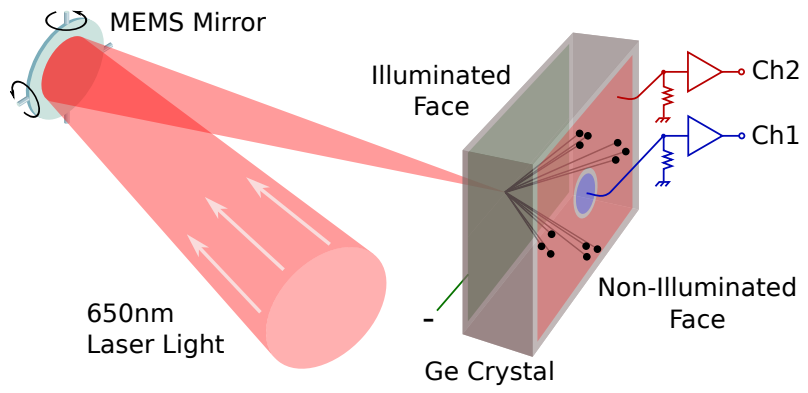

(a) Diagram

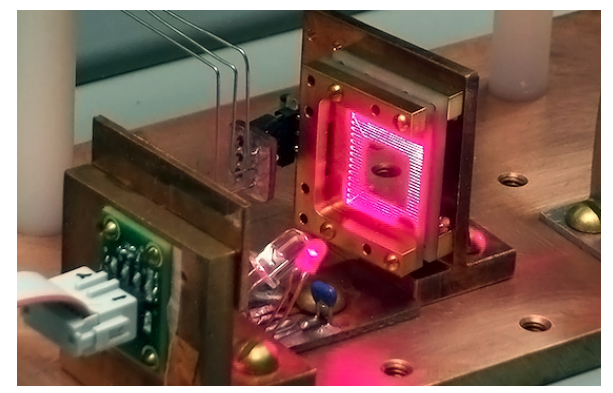

(b) Photo

Figure 6.3: Diagram (a) and photograph (b) of the laser scanning system, consisting of a focused laser beam, electrically controllable MEMS mirror, and crystal sample. The photograph (b) shows the laser beam executing a horizontal raster scan across the crystal surface.

\footnotetext{
${ }^{4}$ Previous designs of the scanning experiment used a piezoelectric actuator to move the mirror rather than a MEMS system. The piezoelectric actuator was found to be inferior due to its heat dissipation, hysteretic effects, and reduced deflection at low temperature. However, in the course of this research a useful model was developed for the purpose of characterizing the behavior of piezoelectric actuators using only electrical measurements. This model is described in Appendix I.
} 


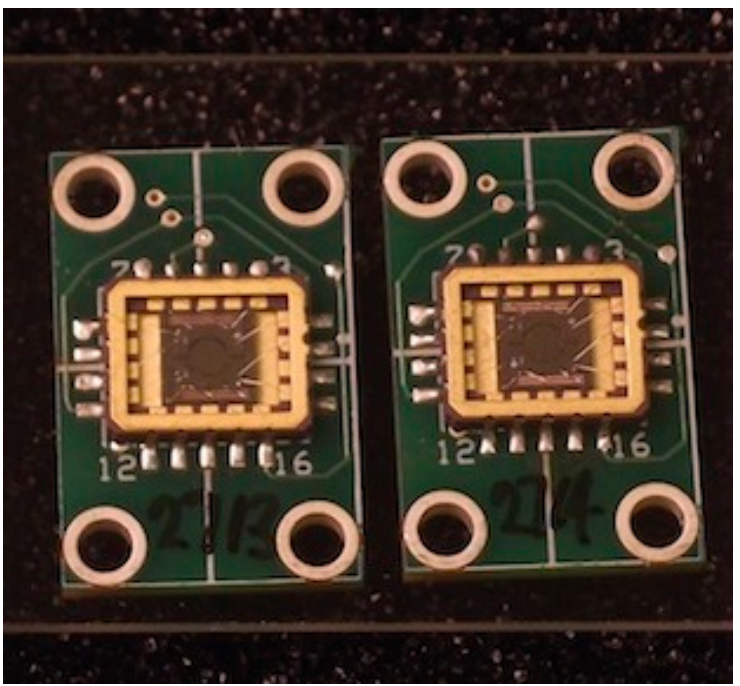

(a) MEMS mirrors mounted on PCBs.

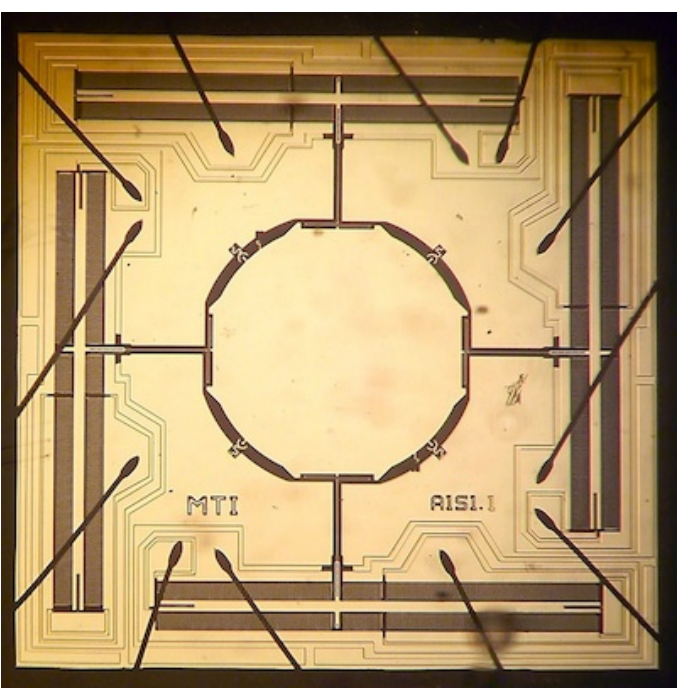

(b) MEMS mirror under microscope.

Figure 6.4: MEMS (Micro Electro-Mechanical Systems) mirrors from Mirrorcle, used to control the position of the laser spot in two dimensions at cryogenic temperatures.

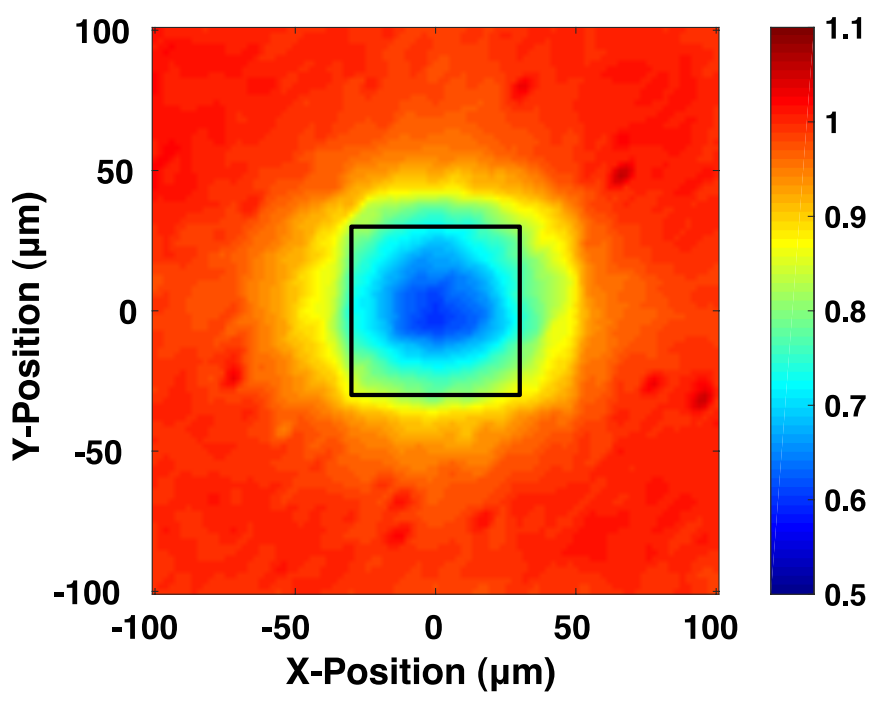

Figure 6.5: Image of the $60 \mu \mathrm{m}$ wide central calibration square taken with the laser scanning system using the channel 2 charge signal. The outline of the calibration square is shown in black. This image is consistent with the laser spot having a Gaussian profile with a FWHM of $70 \mu \mathrm{m}$. 


\subsection{Amplifier Circuit}

When charges are collected by the electrodes, they cause a change in voltage which is amplified by a high-impedance, cryogenic MESFET amplifier circuit, based on the successful past design by Adrian Lee[57]. ${ }^{5}$ The MESFETs used in this circuit are the Sony 3SK165, which was determined by Lee to have good transconductance (about $5 \mathrm{mS}$ ) and low $1 / f$ noise at a temperature of 4K.[60] (See Figure G.23.)

The MESFETs are held on a $4 \mathrm{~K}$ stage separate from the crystal. The signals from the crystal are sent to the gates of the MESFETs through 0.020 inch diameter stainless steel coaxial lines. A simplified schematic for the amplifier circuit used in this experiment is shown in Figure 6.6. The full schematic is shown in Figure G.13 in Appendix G.

The first stage of each signal chain consists of two FETs in a cascode configuration. The Sony 3SK165 MESFETs (Q1 and Q2) act as high impedance, common source, transconductance amplifiers. The drain currents from Q1 and Q2 are sent through the wiring of the He-3 cryostat to the room-temperature Sony 2SK152 JFETs (Q3 and Q4), which act as current buffers. Resistors R9 and R10 transform the current signal back into a voltage signal.

The voltages across R9 and R10 are capacitively coupled to 30dB voltage amplifiers, and sent through three-pole low-pass filters $\left(f_{c}=24 \mathrm{kHz}\right)$ for Nyquist band limiting. ${ }^{6}$ The outputs are read by a National Instruments USB-6009 DAQ at a sampling rate of $48 \mathrm{kHz}$. The gain of the signal chains was chosen so that the noise amplitude at the output of the amplifier was comparable in magnitude to the input noise of the USB-6009 DAQ. This was done in order to maximize the dynamic range of the system without significantly compromising the signal-to-noise ratio.

The room-temperature circuitry allows the drain bias current and the drain-tosource bias voltage of Q1 and Q2 to each be set independently. The drain currents for Q1 and Q2 are set by the current sources, I1 and I2, while capacitors C3-C6

\footnotetext{
${ }^{5}$ MESFETs (metal-semiconductor field-effect transistors) are similar to JFETs in their construction and operation, except that the gate consists of a metal-semiconductor junction rather than a p-n junction.

${ }^{6}$ In addition to the low-bandwidth outputs, the amplifier also has separate high-bandwidth $\left(f_{c}=\right.$ $1 \mathrm{MHz}$ ) outputs with less gain, shown in the full schematic in Figure G.13 in the appendix.
} 


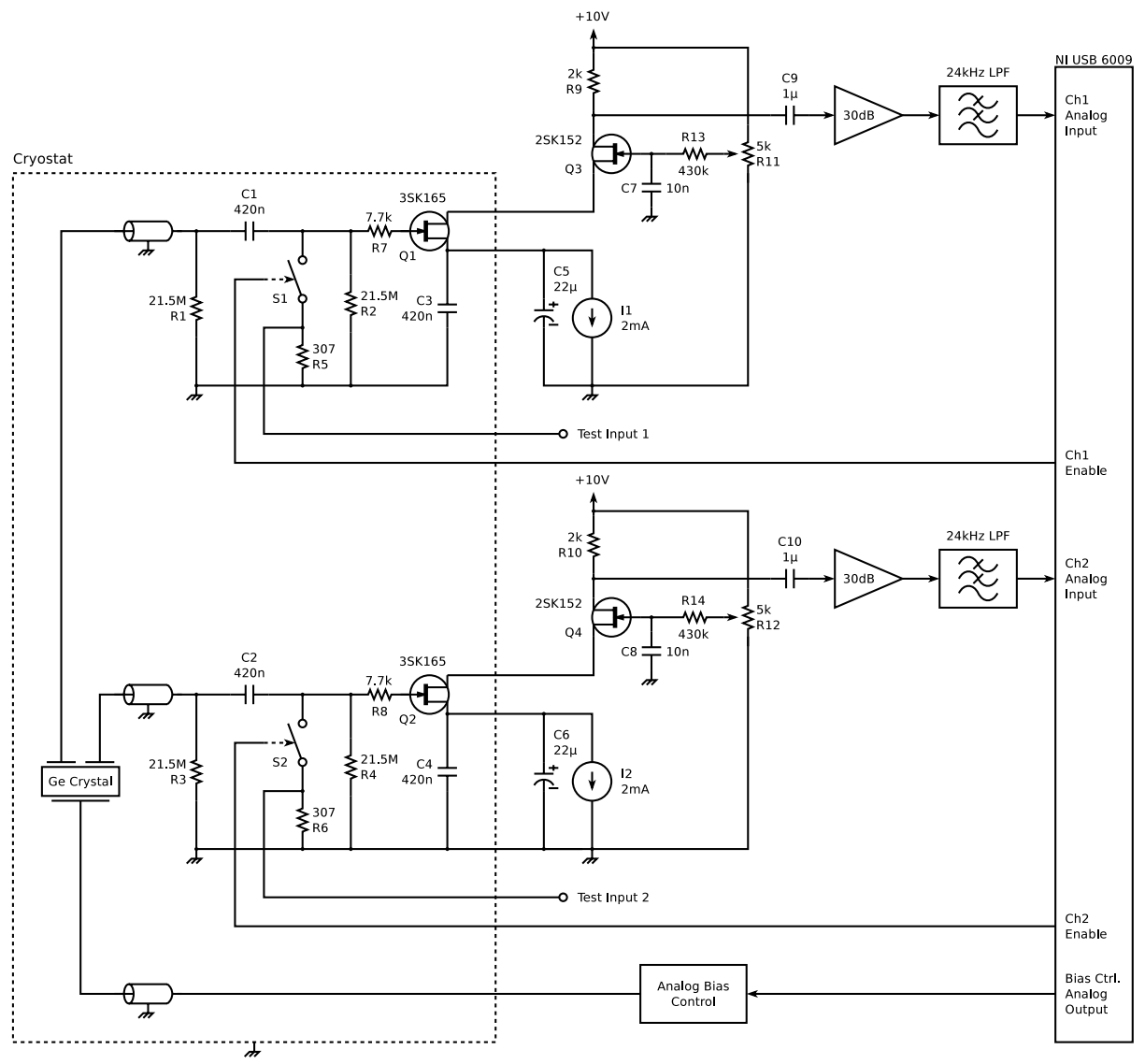

Figure 6.6: Simplified schematic of the amplifier circuit. The dotted line indicates the part of the circuit contained within the cryostat. The rest of the circuit is at room temperature. The full schematic is shown in Figure G.13.

provide an $\mathrm{AC}$ connection from source to ground. The drain-to-source voltages are set by potentiometers R11 and R12. The bias point chosen for both Q1 and Q2 was $I_{\mathrm{D}}=2.0 \mathrm{~mA}, V_{\mathrm{DS}}=0.9 \mathrm{~V}$, and $V_{\mathrm{GS}}=-1.8 \mathrm{~V}$.

Resistors R1-R4 drain the charge to ground from the crystal electrodes and the MESFET gates. The DC-blocking capacitors, C1 and C2, are not necessary under ordinary circumstances. However, on one occasion it was discovered that stray conductance, perhaps from residual soldering flux, caused current leakage from the drain to the gate in $\mathrm{Q} 1$ or $\mathrm{Q} 2$, resulting in an unknown voltage being applied to the crystal 
electrodes. $\mathrm{C} 1$ and $\mathrm{C} 2$ were subsequently added to prevent any unintended current leakage from affecting the crystal bias voltage.

Resistors R7 and R8 prevent instability by forming a single-pole low pass filter in combination with the MESFET gate capacitance with a cutoff frequency between 150 and 250MHz. These resistors were found to be necessary when earlier versions of the amplifier circuit produced oscillations at $315 \mathrm{MHz}$. This instability was a consequence of the high bandwidth of the 3SK165 MESFETs, which were manufactured for use as RF amplifiers in cellphone applications. Because of their relatively low value compared to R1-R4, these gate resistors have a negligible effect on the noise performance or gain in the frequency band of interest.

Both channels include electrically-controllable transistor switches (S1 and S2 in Fig.6.6), made from specially-biased 3SK165 MESFETs, and controlled by TTL signals from the NI USB-6009 DAQ. (See detailed schematic in Fig.G.13) These switches connect the gates of transistors Q1 and Q2 to ground through $307 \Omega$ resistors. The purpose of these switches is to eliminate cross-talk between the high-impedance inputs of the two channels by allowing one channel to be effectively shorted to ground while the voltage of the other channel is measured.

In addition, $\mathrm{S} 1$ and $\mathrm{S} 2$ allow test signals to be directly injected into the gates of Q1 and Q2 from external test inputs at room temperature while the cold amplifier board is at a temperature of $4 \mathrm{~K}$. These test inputs were used to measure the transfer function of the full signal chain, shown in Figure 6.7, while the circuit was at its normal operating temperature of $4 \mathrm{~K}$.

In order to determine the noise performance of the amplifier, 400 noise traces were recorded with the NI USB-6009 DAQ with 1024 samples per trace. The median noise power spectral density was calculated for each channel, and divided by the transfer function to arrive at the input-referenced noise spectrum. The noise spectra of the two channels are shown in Figure 6.8.

In order to determine the quantity of charge collected by the crystal electrodes, it was necessary to estimate the input capacitances of the two amplifier channels. These estimates were calculated using two independent methods: first by measuring the $\mathrm{RC}$ 

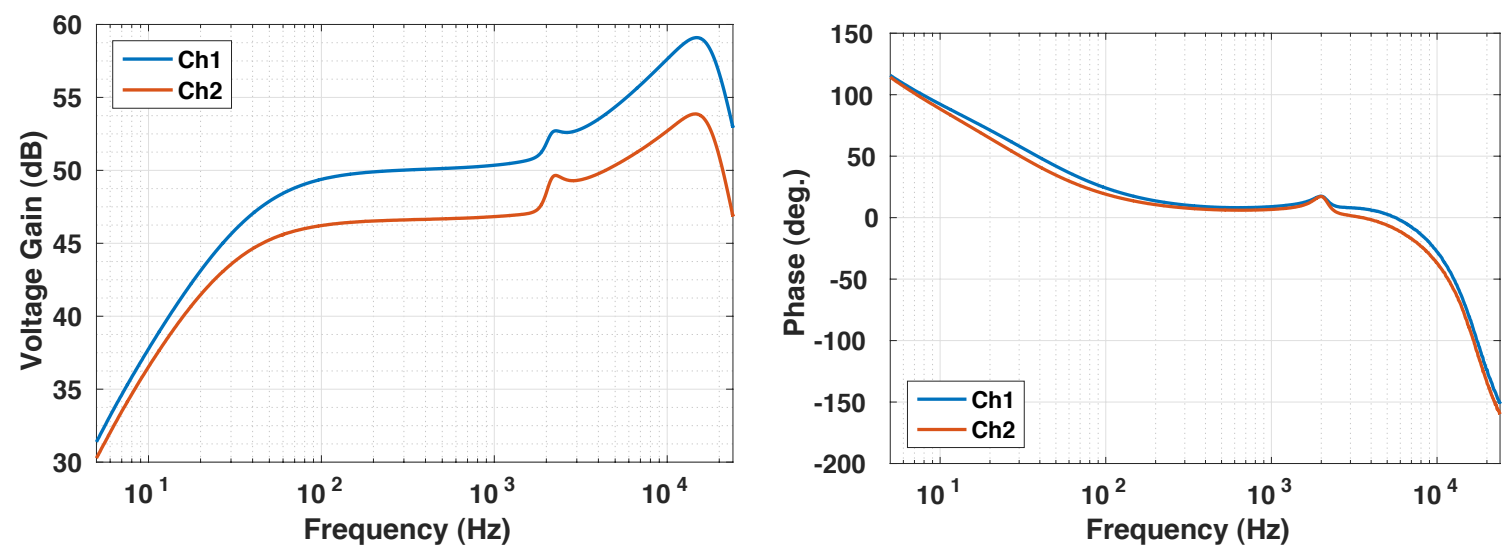

Figure 6.7: Transfer functions for the low-bandwidth outputs of channel 1 and channel 2 , measured by injecting small sine-wave signals into the test inputs of both channels with S1 and S2 closed. The amplitude and phase of the output sine waves were measured as a function of frequency when the cold amplifier board was at a temperature of $4 \mathrm{~K}$.

decay time of an impulse response, and second by summing all known contributions to the input capacitance.

The $\mathrm{RC}$ time constants for both channels were measured at 4K. (See Figure 6.9.) The $\mathrm{RC}$ time constant is determined by the resistors R1-R4 (nominal value of $10 \mathrm{M} \Omega$ each). At $4 \mathrm{~K}$, these resistors were each measured to have $21.5 \mathrm{M} \Omega$ of resistance, giving a parallel resistance of $10.75 \mathrm{M} \Omega$ for each channel. From the RC time constants, the capacitances of channels 1 and 2 were estimated to be $14 \mathrm{pF}$ and $18 \mathrm{pF}$, respectively.

These capacitances were also estimated independently from three main contributions: the crystal electrode capacitance, the coax capacitance, and the MESFET gate capacitance. A parallel-plate approximation for the capacitance of the channel 2 electrode is $3.6 \mathrm{pF}$, assuming a dielectric constant of 16 for germanium. The capacitance of the channel 1 electrode is necessarily less than this.

The 0.020" diameter, stainless steel coax was measured to have a capacitance of $95 \mathrm{pF} / \mathrm{m}$. The lengths of the channel 1 and channel 2 coaxial lines were 4.0" and 5.4" respectively. This gives a capacitance of $10 \mathrm{pF}$ for the channel 1 coax, and $13 \mathrm{pF}$ for the channel 2 coax. 


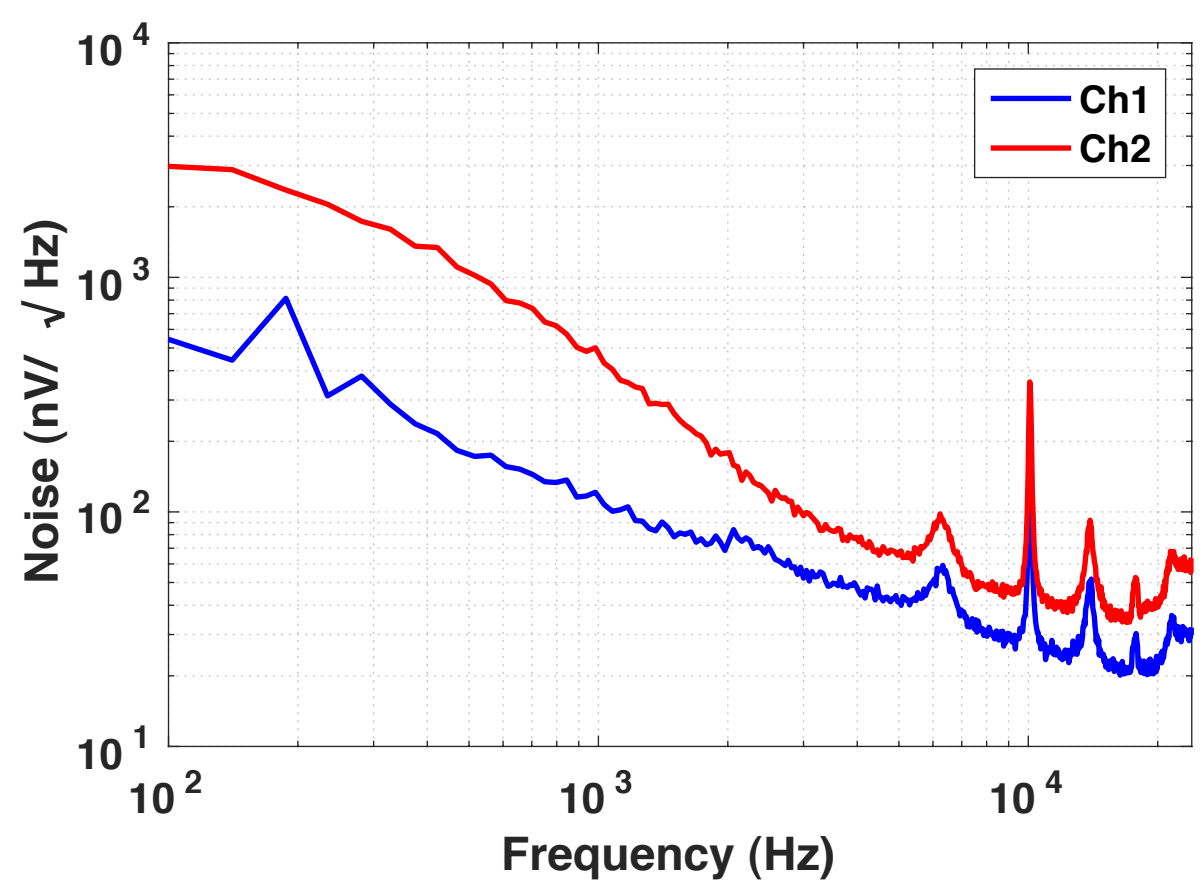

Figure 6.8: Noise spectra for channels 1 and 2 from low-bandwidth output, measured with NI USB-6009 DAQ, referenced to the input, and measured with crystal at $0.6 \mathrm{~K}$ and cold amplifier board at $4 \mathrm{~K}$. The noise spike at $10 \mathrm{kHz}$ is due to local noise on the DAQ. The other noise spikes are aliased harmonics of $10 \mathrm{kHz}$.

According to the datasheet for the 3SK165 MESFETs, the typical input capacitance is $0.5 \mathrm{pF}$, with a maximum value of $1 \mathrm{pF}$. Therefore the total capacitances may be estimated to be $11 \mathrm{pF}$ for $\mathrm{Ch} 1$ and $17 \mathrm{pF}$ for $\mathrm{Ch} 2$, which are close to the estimates derived from the measured RC time constants.

Due to the possible existence of extra stray capacitance not accounted for by the sum of the contributions from all known capacitance sources, the capacitance estimates derived from the RC time constants were used for all subsequent measurements of the charge collection on the channel 1 and channel 2 electrodes. 


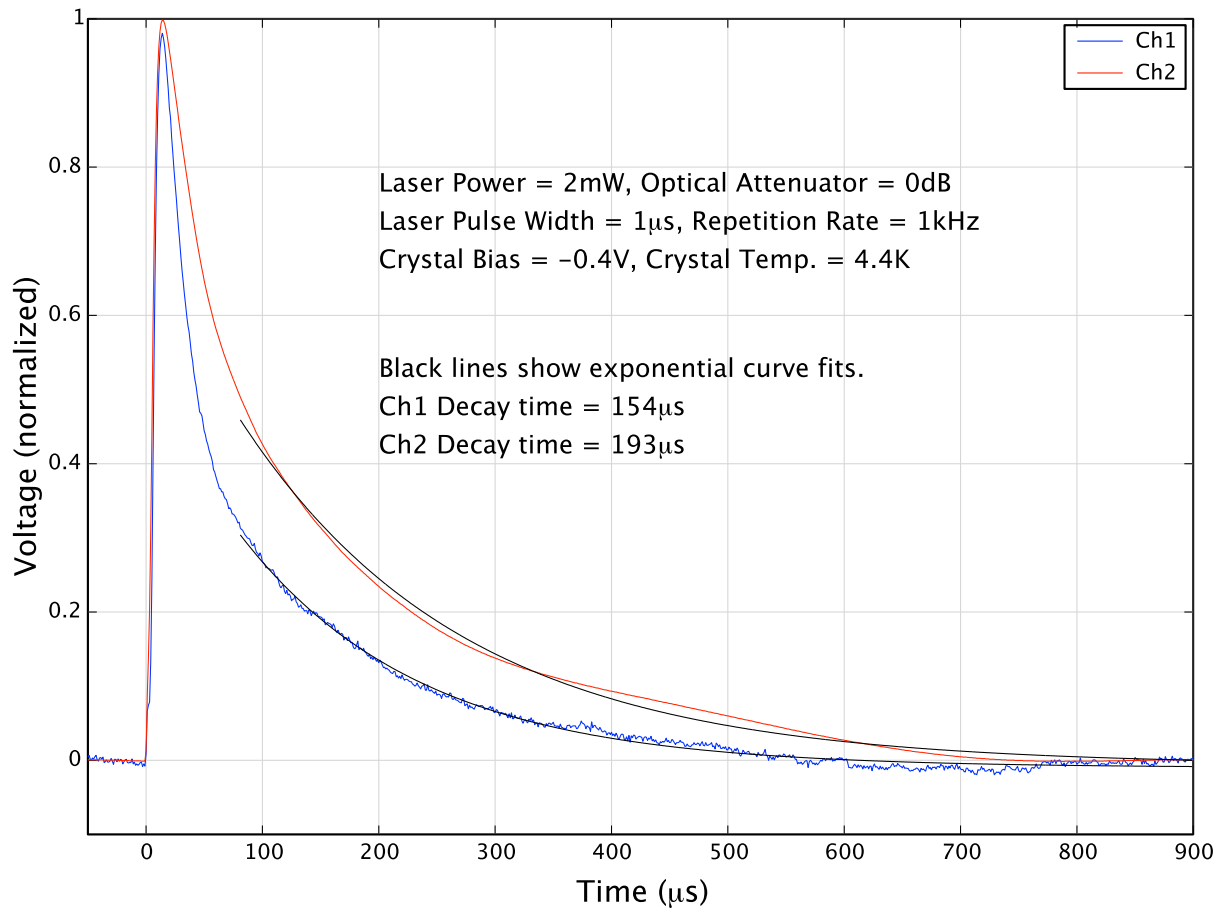

Figure 6.9: Impulse response for channels 1 and 2 from a $1 \mu$ s long laser pulse. These signals were measured using the high-bandwidth output of the amplifier circuit (bypassing the $24 \mathrm{kHz}$ low-pass filter). Assuming a resistance of $10.75 \mathrm{M} \Omega$ per channel, this gives capacitance values of $14 \mathrm{pF}$ and $18 \mathrm{pF}$ for channels 1 and 2 , respectively.

\section{5 ${ }^{3}$ He Cryostat}

A thermal schematic of the ${ }^{3} \mathrm{He}$ cryostat is shown in Figure 6.10. The system contains three sorption refrigeration stages, only two of which are used.[61] Each stage consists of a still for holding liquid ${ }^{3} \mathrm{He}$ or ${ }^{4} \mathrm{He}$ connected through an insulating stainless steel pipe to a pump consisting of a chamber containing an activated charcoal adsorber.

When the system is at equilibrium at $4 \mathrm{~K}$, the helium is all adsorbed onto the high surface area of the activated charcoal. To initiate a cooling cycle, the heat switch for the ${ }^{4} \mathrm{He}$ pump is turned off, and the activated charcoal is heated to $54 \mathrm{~K}$. This causes the ${ }^{4} \mathrm{He}$ to desorb from the charcoal, increasing the vapor pressure in the chamber and causing liquid to form at the $4 \mathrm{~K}$ condensation point between the pump and the still. 


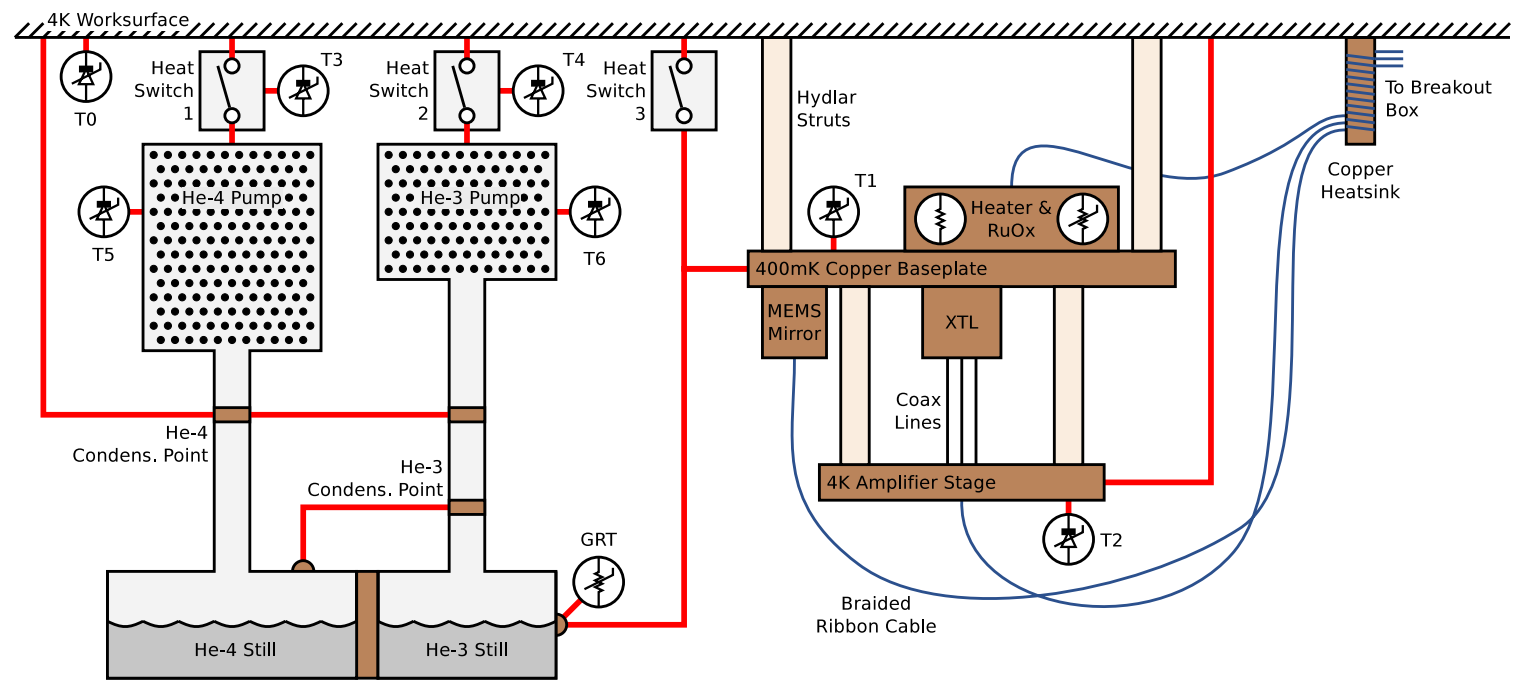

Figure 6.10: Schematic diagram of ${ }^{3} \mathrm{He} /{ }^{4} \mathrm{He}$ cooling system. Red lines show thermal connections. T0-T6 are diode thermometers. GRT is a germanium resistance thermometer, and $\mathrm{RuOx}$ is a ruthenium oxide thermometer. Heat switches 1 and 2 are electrically controllable gas-gap switches. Thermometers T3 and T4 measure the temperatures of internal charcoal adsorbers of the heat switches. Heat switch 3 is a passive gas gap which facilitates the initial cool-down and opens once the worksurface reaches $4 \mathrm{~K}$.

Once the condensed liquid has filled the ${ }^{4} \mathrm{He}$ still, the heater is turned off, and the heat switch turned back on. This causes the vapor pressure of the ${ }^{4} \mathrm{He}$ to drop, depressing the boiling temperature of the liquid and cooling the still

A similar cycle is then performed with the ${ }^{3} \mathrm{He}$ pump, except in this case the cooling power of the liquid ${ }^{4} \mathrm{He}$ is used to condense the ${ }^{3} \mathrm{He}$ vapor into liquid. Once the ${ }^{3} \mathrm{He}$ still is full and the ${ }^{4} \mathrm{He}$ still is empty, the ${ }^{3} \mathrm{He}$ pump is activated, and the ${ }^{3} \mathrm{He}$ still approaches its base temperature of $400 \mathrm{mK}$.

Using a $131 \mathrm{k} \Omega$ heater resistor and a ruthenium oxide thermometer attached to the payload, the load line for the ${ }^{3} \mathrm{He}$ still was measured by Brendan Corcoran, and is plotted in Figure 6.11. At low power levels, the payload temperature was observed to rise by approximately $0.55 \mathrm{~K}$ per $\mathrm{mW}$ of applied heat power.

The ${ }^{4} \mathrm{He}$ stage contains 18 STP liters of ${ }^{4} \mathrm{He}$, and the ${ }^{3} \mathrm{He}$ stage contains 11 STP liters of ${ }^{3} \mathrm{He}$.[61] The product of cooling power and hold-time for the ${ }^{3} \mathrm{He}$ stage was 


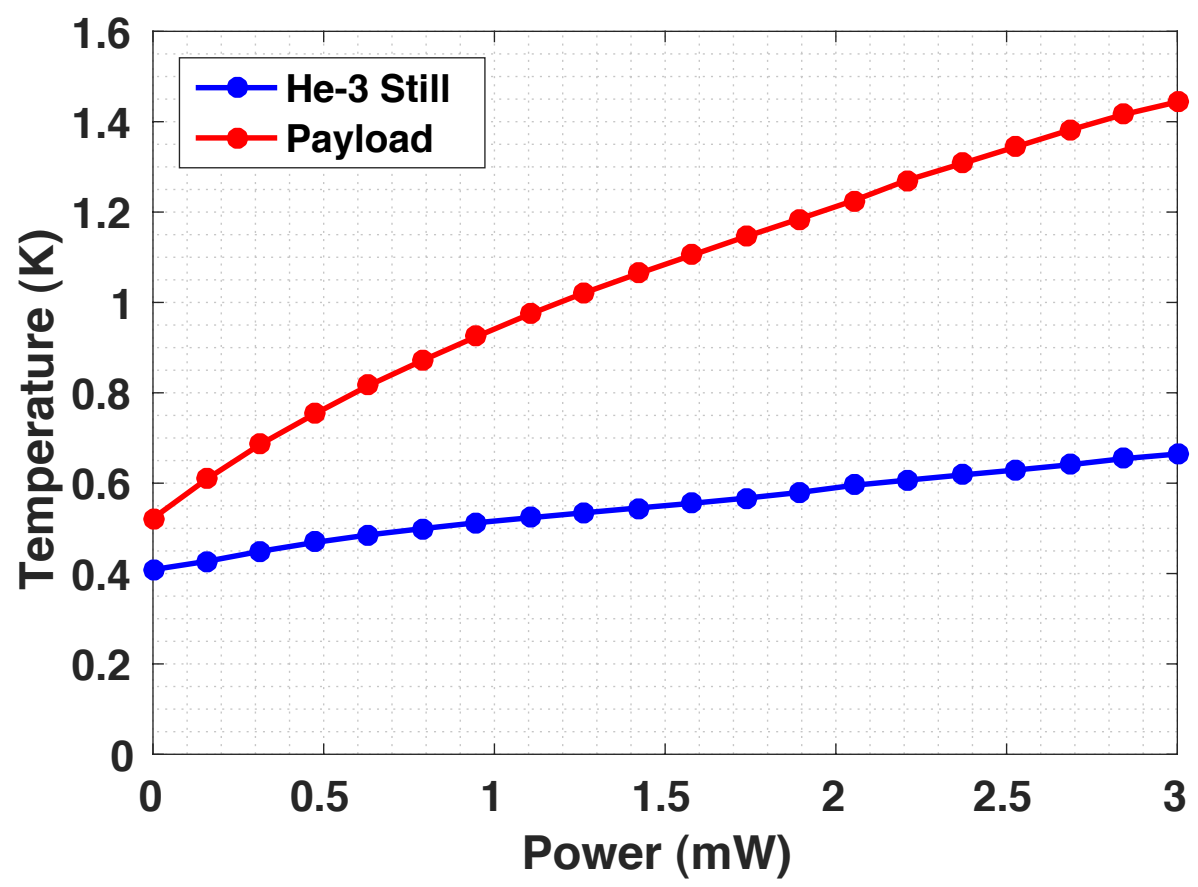

Figure 6.11: Temperature of the ${ }^{3} \mathrm{He}$ still (measured by GRT) and the experimental payload (measured by $\mathrm{RuOx}$ ) as a function of power applied to a heater mounted on the payload.

measured by Corcoran to be $2.56 \mathrm{~mW}$-hours, which is consistent with the measurements by Bhatia in [61]. Based on the 11.6 hour hold-time at base temperature, the net heat leakage to the ${ }^{3} \mathrm{He}$ still is estimated to be $220 \mu \mathrm{W}$.

When the cryogenic amplifier is activated, Q1 and Q2 each dissipate approximately $1.8 \mathrm{~mW}$ of thermal power. Most of this power is conducted to the $4 \mathrm{~K}$ work surface through a copper heat strap. However, some of this power leaks to the copper baseplate of the experimental payload. This leaked power increases the base temperature of the payload to about $630 \mathrm{mK}$ and reduces the hold time to about 6 hours, which is consistent with a heat leakage power of about $200 \mu \mathrm{W}$.

Figure 6.12 shows a photograph of the experimental payload mounted in the ${ }^{3} \mathrm{He}$ cryostat. The optical system is covered with a copper enclosure for shielding electrical noise and $4 \mathrm{~K}$ infrared radiation. The sorption refrigeration stages are the gold-plated cylinders behind the payload. 


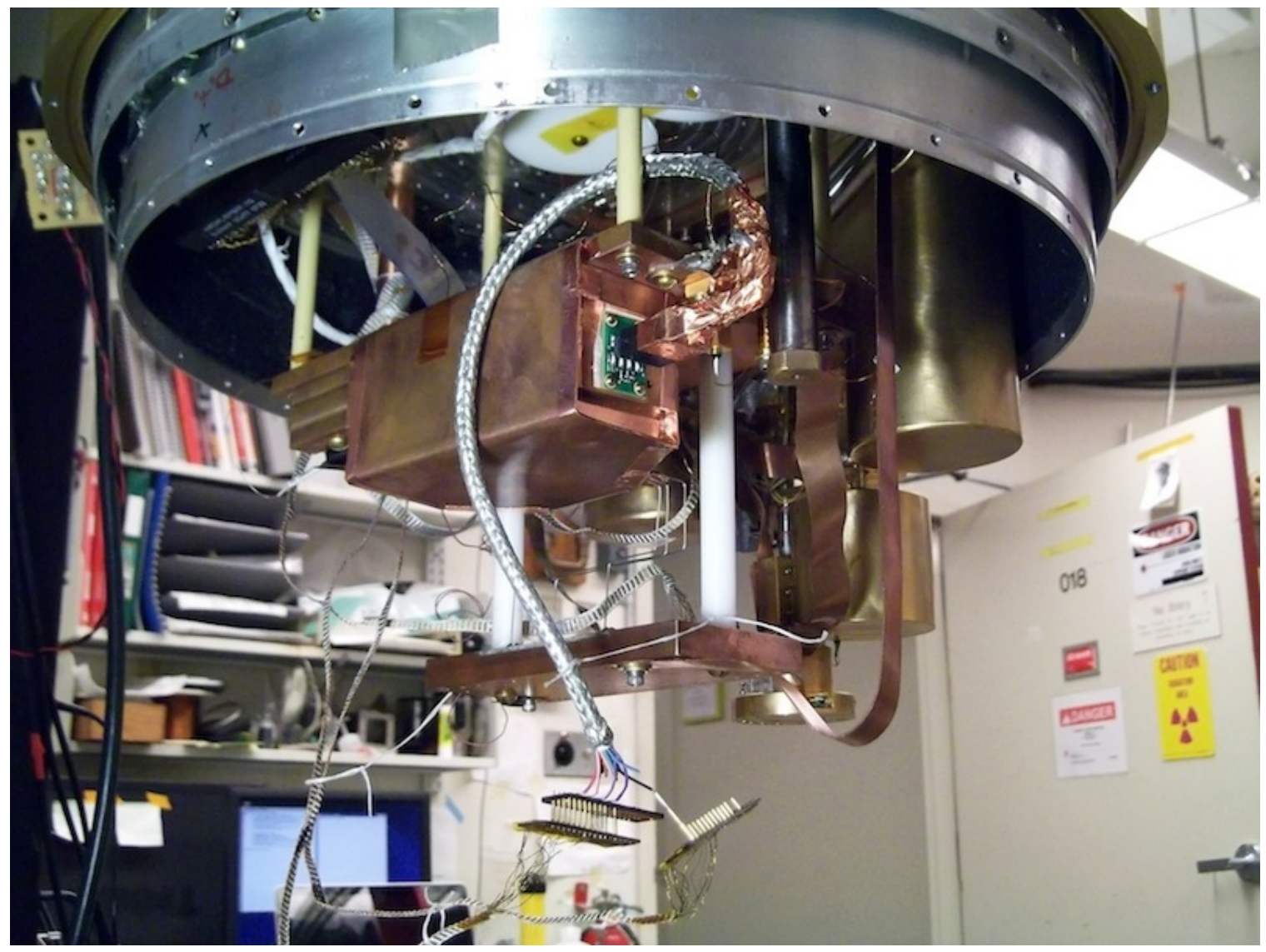

Figure 6.12: Photograph of the experimental payload mounted in the ${ }^{3} \mathrm{He}$ cryostat with vacuum jacket and $4 \mathrm{~K} / 77 \mathrm{~K}$ radiation shields removed.

\subsection{Control System}

The system was controlled using Matlab code on a master control laptop. (See Appendix J) All of the laboratory instruments were connected to the laptop through either USB or GPIB. The control laptop was connected to the internet, which allowed remote status reports and control of the system.

The ${ }^{3} \mathrm{He}$ cryostat was controlled by a separate computer which was also connected to the internet. The control laptop was able to execute remote commands on the fridge control computer through the internet connection. 


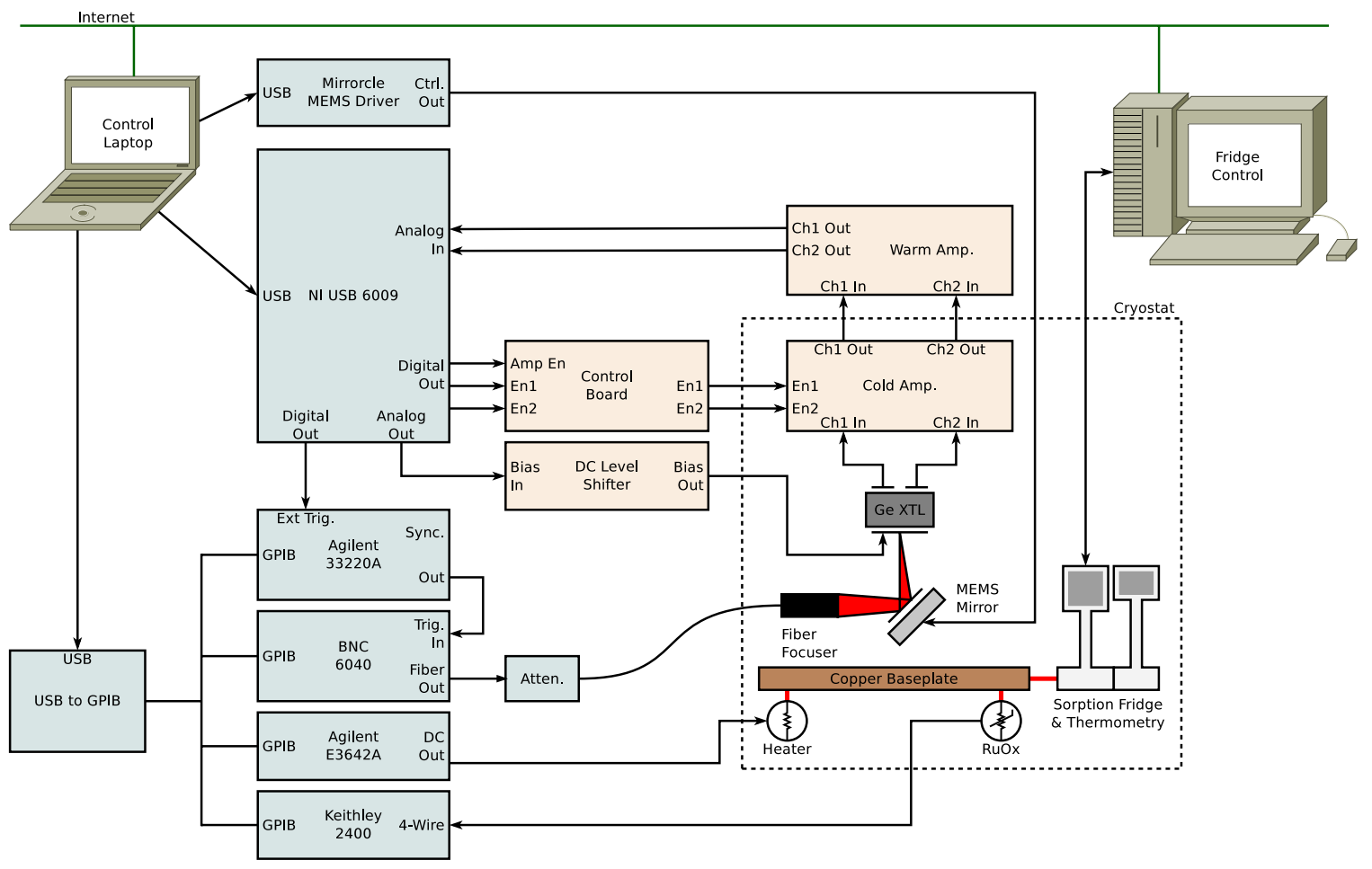

Figure 6.13: Simplified block diagram of the control system. 


\section{Chapter 7}

\section{Experimental Methods}

\subsection{Neutralization}

The ${ }^{3} \mathrm{He}$ cryostat requires approximately two hours to cool from $4 \mathrm{~K}$ to base temperature. During this time, the crystal electrodes are grounded, and the crystal is illuminated with diffuse laser light by shining the laser spot onto the plastic housing of an LED mounted in front of the crystal holder (see Figure 6.3b). The diffuse illumination is then followed by a raster scan with zero bias. The purpose of this process is neutralize the crystal, i.e. to ensure that no charge carriers remained bound by shallow traps created by impurities or defects either in the crystal bulk or on the surfaces. (See the work by Kyle Sundqvist in [4] for a detailed discussion of charge trapping.)

\subsection{Scanning Process}

Once the crystal is at base temperature, the amplifier is turned on and the scanning process is commenced. Due to the trapping of charge by impurities, it was found to be necessary to flash the crystal with laser light at zero bias between each measurement in order to ensure the crystal remained in its neutralized state. For the flash, the laser is pointed at the LED to diffusively illuminate the crystal for a period of $10 \mathrm{~ms}$. 
The set of steps executed at each pixel location are:

1. Record time and temperatures.

2. Set bias voltage to zero.

3. Move mirror to flash location.

4. Flash crystal.

5. Move mirror to pixel location.

6. Turn on bias voltage.

7. Enable channel 1.

8. Apply laser pulse train to crystal. Record channel 1 voltage.

9. Disable channel 1. Enable channel 2.

10. Apply laser pulse train to crystal. Record channel 2 voltage.

11. Disable channel 2.

For more details regarding the scanning process, see Appendix J which contains the Matlab code and a description of its operation.

\subsection{Maximum-Likelihood Fits}

At each pixel location in the two-dimensional scan, a train of 20 laser pulses is applied to the crystal at a rate of one pulse every $1053.1 \mu \mathrm{s} .{ }^{1}$ The amplified voltage pulses from each channel are recorded by the NI USB-6009 DAQ.

An example of the raw data collected from a scan of the crystal is shown in Figure 7.1. The voltage amplitudes shown in the two plots on the left refer to the pulse amplitudes measured at the low-bandwidth outputs of the amplifier circuit.

\footnotetext{
${ }^{1}$ This period was chosen so that the gap between the harmonics in the Fourier Series of the signal would coincide with the prominent noise spike at $10 \mathrm{kHz}$, thereby reducing the overlap between the frequency spectra of the signal and noise.
} 


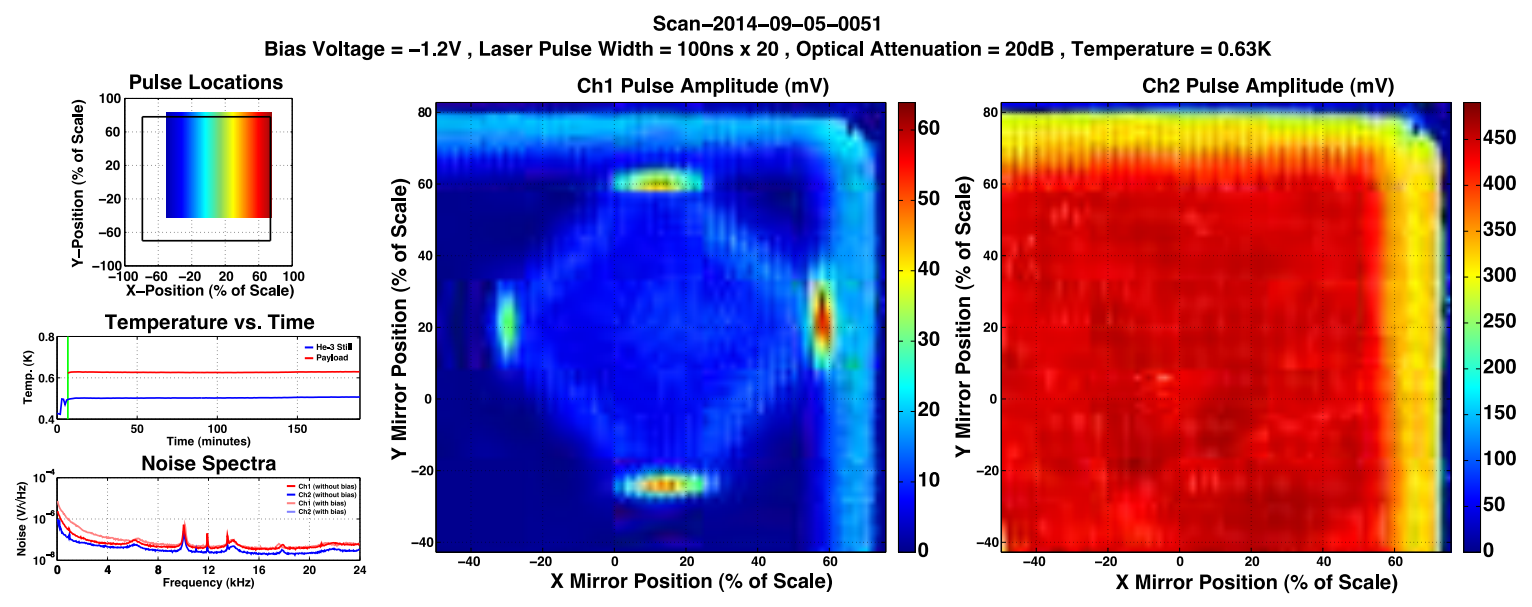

Figure 7.1: Example of raw data from a scan of the crystal.

Once each scan is complete, the mean of all pulse trains for each channel is calculated to form a pair of templates. Figure 7.2 shows the templates generated from the same scan as that in Figure 7.1. The templates are both normalized to have a peak-to-peak amplitude of 1.

Because the start-time of the pulse train is known, the only unknown variable that determines its shape is a linear scale factor which is proportional to the total quantity of charge collected during each pulse. The scale factor for each pulse train, as a function of position, is therefore a quantity of interest.

The scale factor for a particular pulse train is defined to be the factor which, when multiplied by the template, gives the closest fit. Because the noise spectrum is not white, closeness of fit is defined by a weighted least-squares fit in the frequency domain, with the weighting factor for each frequency bin being proportional to the inverse of the measured noise power-spectral-density in that bin. Appendix H provides a derivation this formula under the assumption of stationary, Gaussian noise.

\subsection{Pulse Gain}

The maximum-likelihood fitting algorithm described in $\S 7.3$ provides an estimate of the amplitudes of the pulses measured at the low-bandwidth output of the amplifier. 


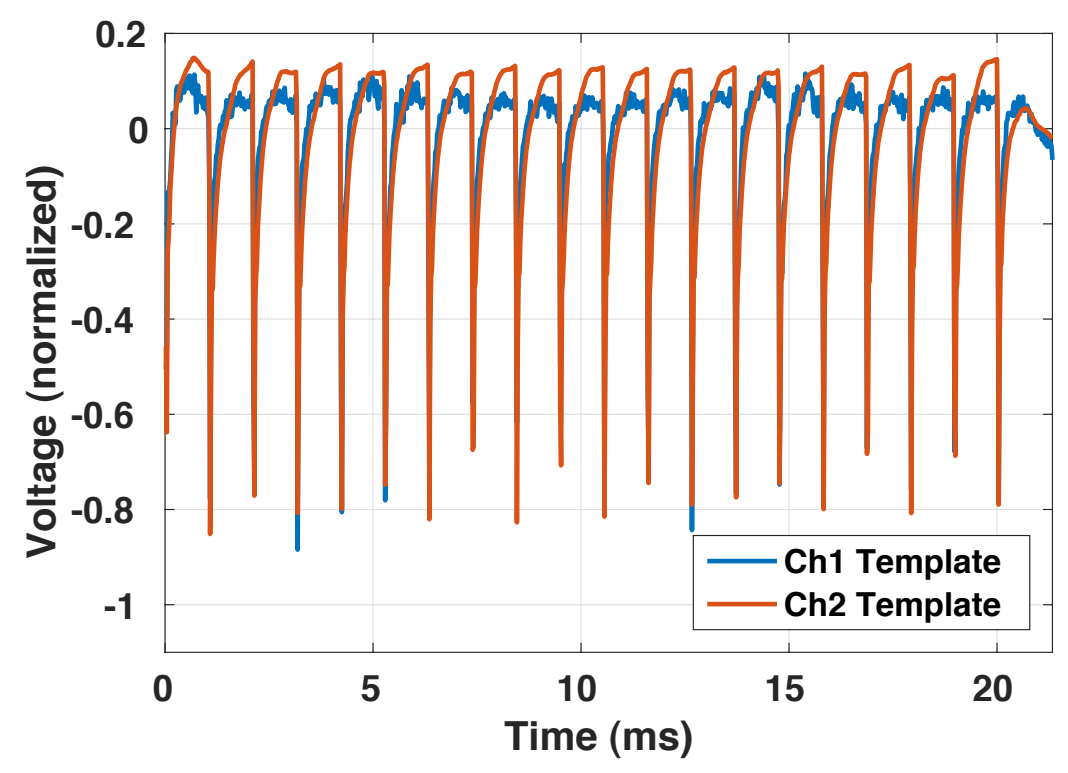

Figure 7.2: Low-bandwidth pulse train templates.

To estimate the total charge collected by the crystal electrodes during each pulse, it is necessary to estimate the input voltage for each channel. Assuming the amplifier response is linear, the input amplitude may be determined from the output pulse if the overall pulse gain of the amplifier circuit is known. However, because the amplifier gain is a function of frequency, it is necessary to utilize the known amplitude and phase of the full transfer function for each channel.

The input pulse shape for each channel was assumed to be a decaying exponential. The known amplifier transfer functions were applied to these pulses to generate theoretical output responses. The amplitude and decay time of the exponentials were then varied to find a least-squares fit to the unit-amplitude template for each channel. ${ }^{2}$ These fits are shown in Figure 7.3.

The pulse gain for each channel is therefore given by the inverse of the best-fit amplitudes of the exponential pulses. These gain factors were found to be $G_{1}=612$ and $G_{2}=359$ for channel 1 and channel 2, respectively.

\footnotetext{
${ }^{2}$ The pulse trains were wrapped in the time domain modulo the pulse period. This effectively increased the sample rate, and provided 20 times more averaging.
} 
Note also that the best estimates for the exponential decay times are larger than the decay times measured from the high-bandwith outputs, shown in Figure 6.9, by $14 \%$ for channel 1 , and $61 \%$ for channel 2 .
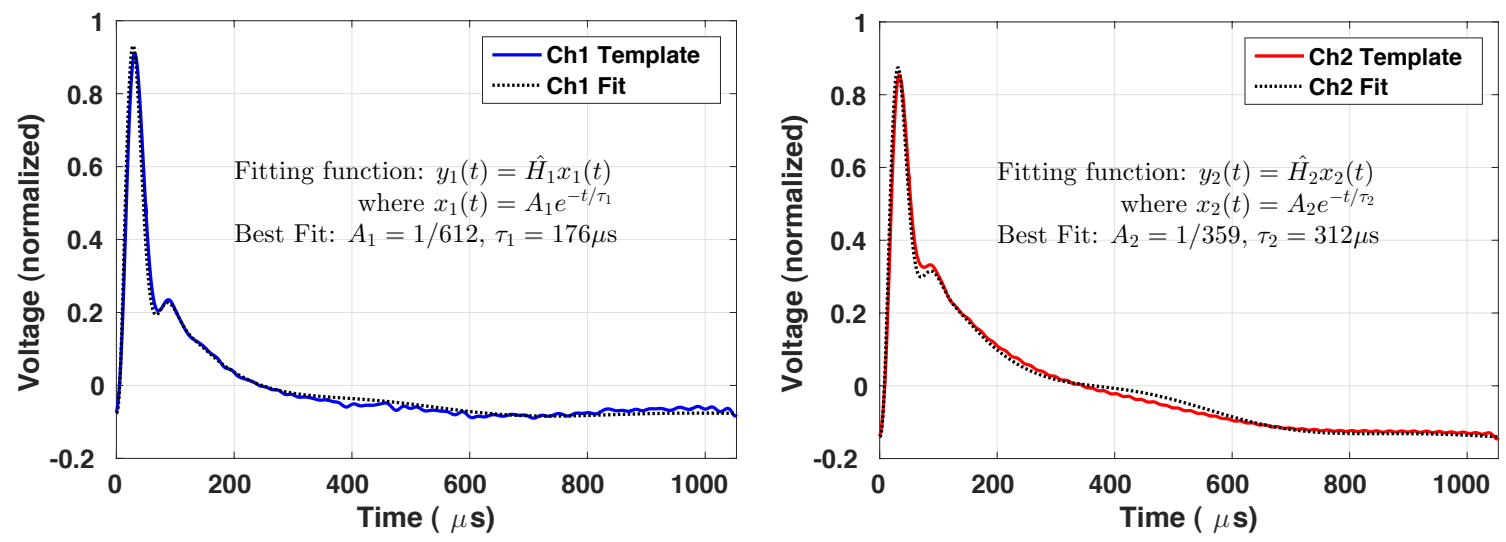

Figure 7.3: Least-squares fits to the wrapped templates for channel 1 and channel 2. The templates were scaled to have an amplitude of 1 . The fitting functions were generated by applying the known transfer function for each channel to an exponential pulse.

\subsection{Charge Collection Estimates}

We may use the amplifier pulse gain and output pulse amplitude to estimate the total charge collected by the channel 1 and channel 2 electrodes. The maximum amplitudes of the output pulses are roughly $60 \mathrm{mV}$ for channel 1 and $450 \mathrm{mV}$ for channel 2. (See Figure 7.1.) From the estimates of the pulse gains, these correspond to input pulse amplitudes of roughly $98 \mu \mathrm{V}$ for channel 1 , and $1.3 \mathrm{mV}$ for channel 2 . Using $14 \mathrm{pF}$ and $18 \mathrm{pF}$ for the capacitances of these two channels (see $\S 6.4$ ), we estimate the total charge collected to be 8,600 and 140,000 electrons per pulse for channels 1 and 2, respectively.

We may compare these numbers to the estimated number of electron-hole pairs generated by each laser pulse. The laser pulses have a peak power of $20 \mu \mathrm{W}$, and a duration of 100ns, giving a total pulse energy of $12.6 \mathrm{MeV}$. Each $650 \mathrm{~nm}$ photon 
carries an energy of $1.9 \mathrm{eV}$. Therefore, each laser pulse contains an average of $6.5 \cdot 10^{6}$ photons.

However, about $20 \%$ of the crystal surface is covered by reflective aluminum, and we estimate the remaining surface to have a power reflectivity of $40 \%$ due to the change in index of refraction between vacuum, amorphous silicon, and germanium. ${ }^{3}$ Therefore, we estimate about $3.1 \cdot 10^{6}$ photons penetrate into the germanium per pulse. Because each photon can generate at most one electron-hole pair, this number represents the maximum number of charge carriers which could be generated by such a laser pulse, assuming $100 \%$ efficiency.

We see that the charge collected by the channel 2 electrode represents only about $5 \%$ of this number. This low efficiency is likely due to the occurrence of recombination in the initial cloud of electron-hole pairs generated by the laser pulse. We have not yet attempted a calculation to reproduce the observed efficiency factor, as the presence of complex effects, such as electron-hole plasma physics, or the generation of excitons, makes this initial process very difficult to model or predict.

\subsection{Charge Noise}

There are four main noise contributions to the measured charge signals: shot noise, electronic noise, systematic error, and hysteresis.

The shot noise may be estimated by assuming that the number of charges collected during each pulse follows a Poisson distribution. Because the variance of a Poisson distribution is equal to its mean, the contribution to the standard deviation in the number of collected charges is equal to the square root of the number of charges collected during each pulse. For a pulse train consisting of $N_{\text {pulse }}$ pulses, the variance is reduced by a factor of $N_{\text {pulse }}$. Therefore, the shot noise contribution to the charge collected by the two channels is:

\footnotetext{
${ }^{3}$ Reflectivity calculated using the Luxpop online calculator, assuming 40nm of aSi on Ge.[62]
} 


$$
\begin{aligned}
& \sigma_{q 1}^{\text {(shot) }}=e \frac{\sqrt{\left|Q_{1} / e\right|}}{\sqrt{N_{\text {pulse }}}} \\
& \sigma_{q 2}^{\text {(shot) }}=e \frac{\sqrt{\left|Q_{2} / e\right|}}{\sqrt{N_{\text {pulse }}}}
\end{aligned}
$$

where $e$ is the electron charge, and where $Q_{1}$ and $Q_{2}$ are the quantities of charge collected by channel 1 and channel 2, respectively. The maximum shot noise occurs where the charge collection is largest. For the maximum charge collection quoted in $\S 7.5$, these contributions are:

$$
\begin{aligned}
\sigma_{q 1}^{\text {(shot) }} & =e \frac{\sqrt{8600}}{\sqrt{20}}=20 e \\
\sigma_{q 2}^{\text {(shot) }} & =e \frac{\sqrt{140000}}{\sqrt{20}}=84 e
\end{aligned}
$$

The electronic noise comes from both thermal Johnson noise, and other sources of voltage noise both internal and external to the amplifier system. The noise powerspectral-density for each channel is known, and is observed to be stable throughout each scan. According to Appendix H, the contribution from electronic noise is given by:

$$
\begin{aligned}
\sigma_{o v 1}^{\text {(elec.) }} & =\left(\int_{-\infty}^{\infty} d \nu \frac{R_{1}^{*}(\nu) R_{1}(\nu)}{\sigma_{n 1}(\nu)}\right)^{-1 / 2}=129 \mu \mathrm{V} \\
\sigma_{o v 2}^{\text {(elec. })} & =\left(\int_{-\infty}^{\infty} d \nu \frac{R_{2}^{*}(\nu) R_{2}(\nu)}{\sigma_{n 2}(\nu)}\right)^{-1 / 2}=114 \mu \mathrm{V}
\end{aligned}
$$

where $\sigma_{o v 1}^{\text {(elec.) }}$ and $\sigma_{o v 2}^{\text {(elec.) }}$ are the standard deviations in the fit parameters for the voltage amplitude of the output pulses, $R_{1}$ and $R_{2}$ are the Fourier transforms of the fit templates, and $\sigma_{n 1}$ and $\sigma_{n 2}$ are the electronic noise power-spectral-density functions for channels 1 and 2 referenced to the amplifier output. We may use the pulse gain, $G$, and capacitance, $C$, for each channel to compute the equivalent input charge noise for channel $1, \sigma_{q 1}^{\text {(elec.) }}$, and channel $2, \sigma_{q 1}^{\text {(elec.) }}$ : 


$$
\begin{aligned}
\sigma_{q 1}^{\text {(elec. })} & =\frac{\sigma_{o v 1}^{\text {(elec. }}}{G_{1}} C_{1}=18 e \\
\sigma_{q 2}^{\text {(elec. })} & =\frac{\sigma_{o v 2}^{\text {(elec. }}}{G_{2}} C_{2}=36 e
\end{aligned}
$$

Several sources contribute to the systematic error in the estimate of the total charge collection. The most prominent is the systematic error in the estimate of the capacitance of each channel. As discussed in $§ 7.4$, the two different estimates of this capacitance from the exponential decay time differ by $14 \%$ for channel 1 and $61 \%$ for channel 2. (See Figures 7.3 and 6.9.) The uncertainty in the total charge collection is directly proportional to the uncertainty in the input capacitance of each channel. However, since these capacitances are constant, their uncertainty only affects the absolute charge calibration, and has no effect on relative measurements between pulses from different crystal locations.

An additional systematic error was observed in the overall signal amplitude as a function of position. The channel 2 charge collection shows a standard deviation of roughly $3.4 \%$ in pulse amplitude as a function of position in regions where the pulse amplitude should be uniform. This variation is visible as a wavelike pattern the lower left of Figure 7.1. Because this pattern was observed to be constant throughout all of the crystal scans, we hypothesize that it is caused by a combination of a variation in the overlap between the mesh electrode and the laser beam profile, and a spatial variation in the fractional coverage by the aluminum mesh electrode on the illuminated face of the crystal.

\subsection{Raster Scan Patterns and Hysteresis}

The largest systematic error in the charge measurement came from hysteresis. It was discovered that the crystal demonstrated a memory effect; a charge pulse with a large amplitude was found to suppress the amplitudes of the succeeding pulses. This effect was reduced by flashing the crystal between the measurement of each pixel, but some 
residual hysteresis remained. The crystal surface was scanned in both horizontal and vertical zig-zag raster patterns, shown in Figure 7.4. Even with the intermediate flashes, the pulse heights were observed to vary by as much as $30 \%$ depending on the direction of the scan, resulting in the notable striping patterns visible Figure 7.4.

In order to mitigate the remaining hysteresis, the results from both a horizontal and a vertical raster scan pattern were averaged together, and a two-dimensional filter was applied which eliminated the highest spatial frequencies contained in the image. (See Figure 7.5.)

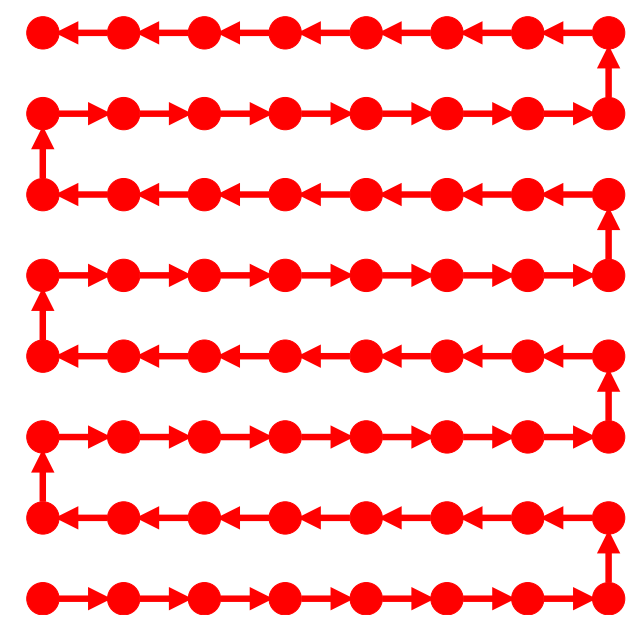

(a) Horizontal raster scan pattern.

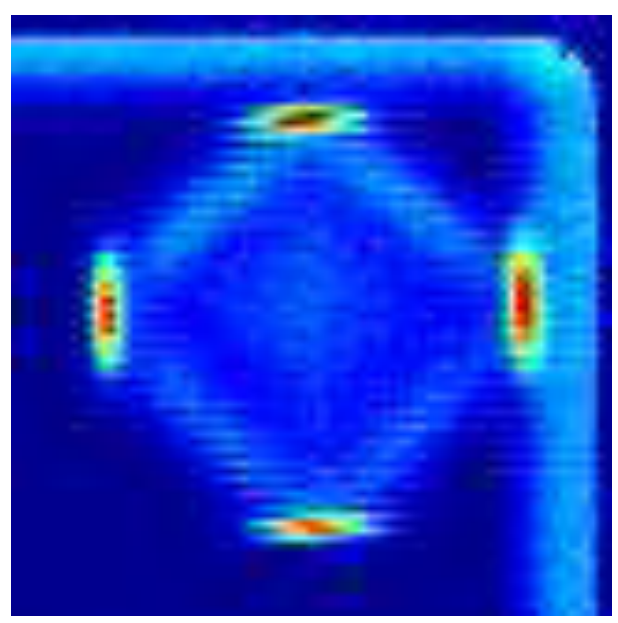

(c) Horizontal raster scan.

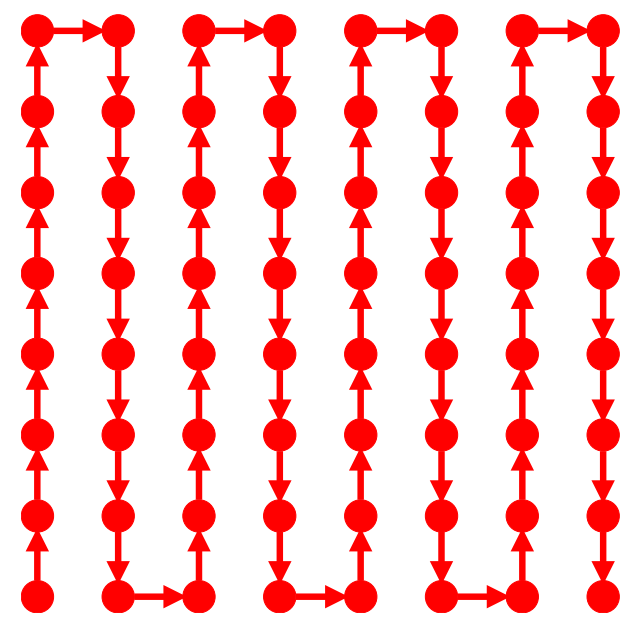

(b) Vertical raster scan pattern.

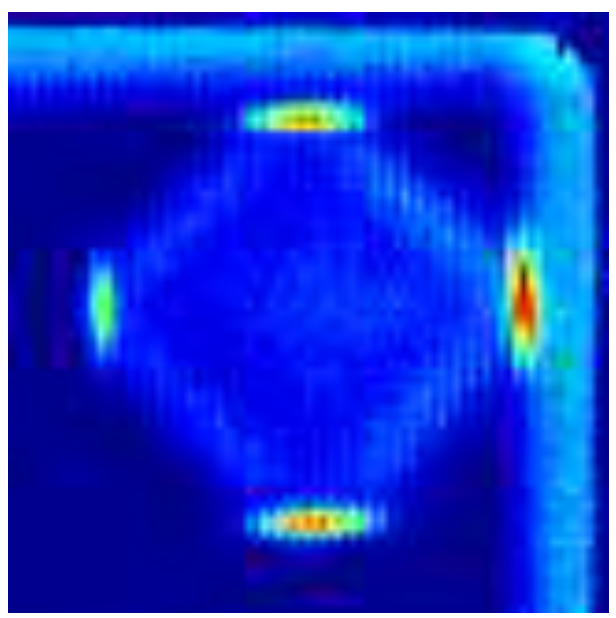

(d) Vertical raster scan.

Figure 7.4: Examples of horizontal (a) and vertical (b) scan patterns. Note the hysteretic effects visible in the charge collection (c) and (d). 


\subsection{Edge Effects}

Also apparent in Figure 7.4 (c) and (d) is an increase in the channel 1 pulse amplitude for laser positions close to the edge of the crystal. This pattern was consistent across various bias conditions on the crystal, and its cause is currently unknown.

In order to avoid contamination of the charge density pattern from this edge effect, an average of the edge signal was taken across the top and right edges in regions where the charge density pattern was presumed to be zero. These averages were then subtracted from the image across the entire top and right edges.

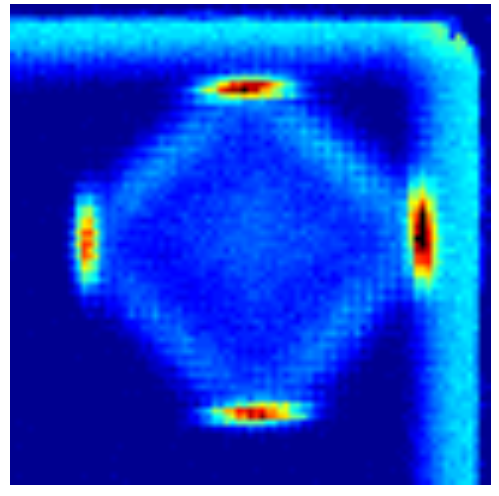

(a) Combined scans.

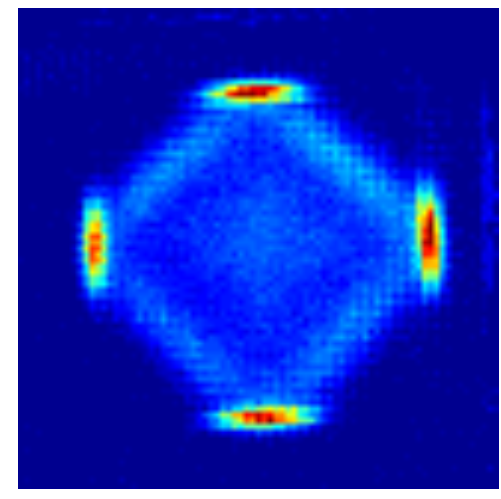

(b) Edge effects subtracted.

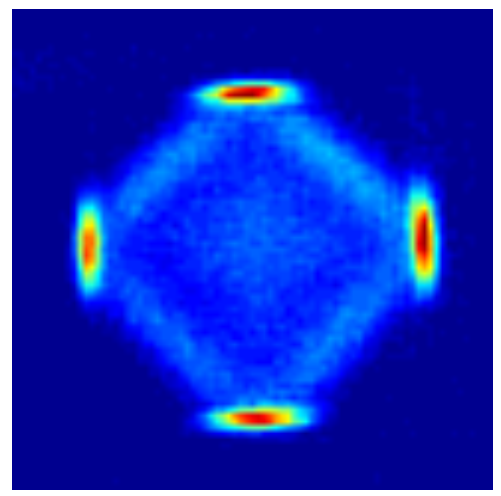

(c) Filtered.

Figure 7.5: Steps for processing of charge density scans. (a) Sum formed from one horizontal and one vertical scan, taken sequentially. (b) Edge effects subtracted. (c) 2-D filter applied to image to remove high spatial frequencies.

\subsection{Position Calibration}

Four circular calibration spots were placed in the corners of the electrode pattern on the illuminated face of the crystal, and one spot was placed in the center. The calibration spots in the corners are circles with a diameter of $160 \mu \mathrm{m}$. The calibration spot in the center is a square with a side length of $60 \mu \mathrm{m}$. The center-to-center separation between the calibration spots in the corners is $8400 \mu \mathrm{m}$. 
The locations of these spots in the mirror coordinate system were used to create a linear map to the physical coordinate system on the surface of the crystal. The calibration spot in the center of the illuminated face was used to define the origin of the crystal coordinate system. The $\mathrm{x}$ and $\mathrm{y}$ directions of this coordinate system are shown by the annotations in Figure 6.2.
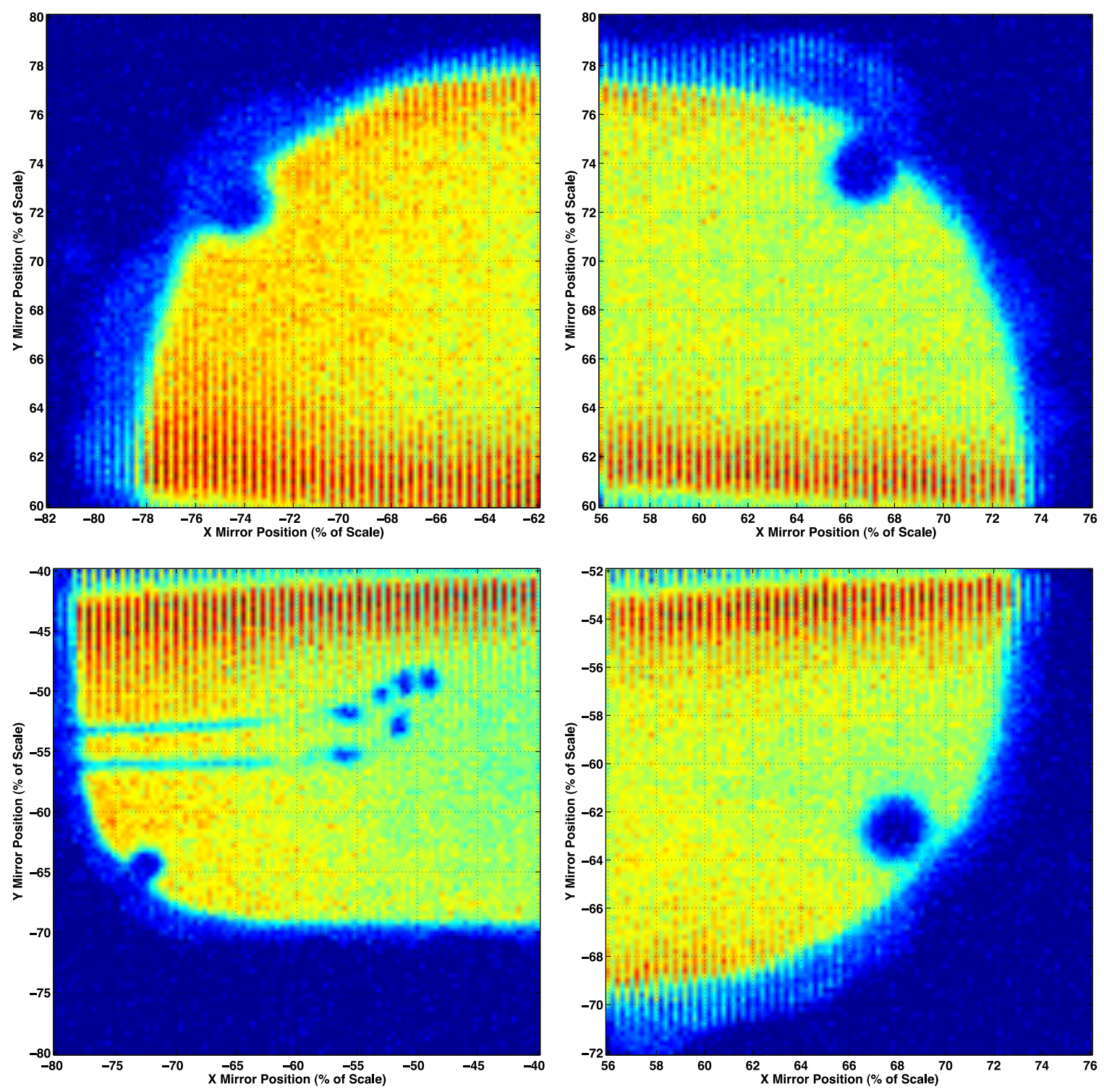

Figure 7.6: Images of the calibration spots in the four corners of the crystal. Also note the shadows of the wire-bonds which are visible in the lower left image. 


\subsection{Inter-Valley Scattering Length}

In order to estimate the inter-valley scattering length, we divided the electron charge density pattern into different regions based on whether the electrons in those regions did or did not undergo inter-valley scattering. (See Figure 7.7.) Based on the charge collected in these regions, and using Equation 5.14, we defined the inter-valley scattering length to be:

$$
\lambda_{\mathrm{IV}}=-\frac{x}{\ln p(x)}=-\frac{x}{\ln \left(\frac{Q_{\mathrm{ns}}}{Q_{\mathrm{tot}}}\right)}
$$

where $x=3.89 \mathrm{~mm}$ is the crystal thickness, $p(x)=Q_{\mathrm{ns}} / Q_{\mathrm{tot}}$ is the probability of an electron traversing the crystal without undergoing inver-valley scattering, $Q_{\mathrm{ns}}$ is the quantity of charge which did not undergo inter-valley scattering, and $Q_{\text {tot }}$ is the total quantity of charge in the 2D charge density pattern.

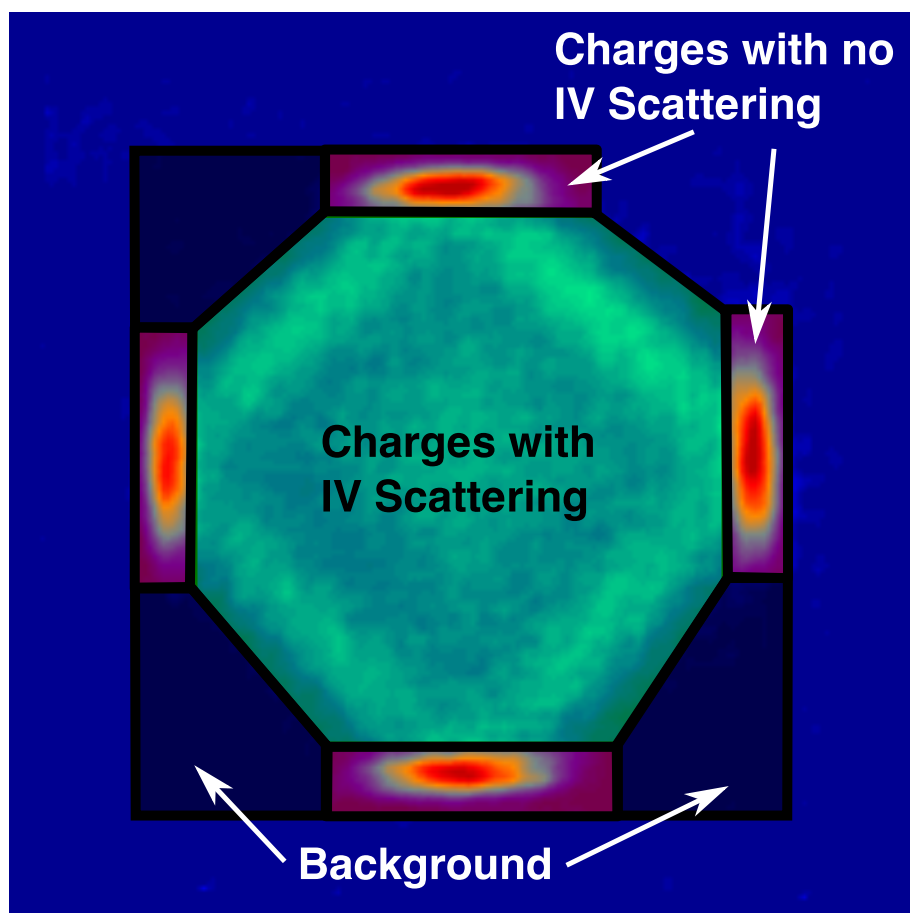

Figure 7.7: Regions in the 2D charge density pattern used to define populations of charge both with inter-valley scattering events (green shaded region) and without inter-valley scattering events (red shaded regions). The mean signal in the gray boxes was used to define the background, which was subtracted from the rest of the charge density pattern. 


\section{Chapter 8}

\section{Experimental Results}

\subsection{Two-Dimensional Charge Density}

Experimental measurements of the two-dimensional charge patterns for a variety of electric field strengths are shown in Figure 8.1 (a) for holes and Figure 8.2 (a) for electrons. For comparison, Figures 8.1 (b) and 8.2 (b) show the charge density patterns generated from the Redl simulation for the same electric field strengths. Figures 8.1 (c) and 8.2 (c) show a cross-sectional comparison between the simulated and measured charge distributions.

The effects of the electron mass anisotropy are clearly evident in Figure 8.2 (a). At low electric fields (less than $\sim 3 \mathrm{~V} / \mathrm{cm}$ ), the electrons separate into four groups, each corresponding to one of the conduction band minima, or valleys. At high electric fields (greater than $\sim 4 \mathrm{~V} / \mathrm{cm}$ ), the inter-valley scattering induced by phonon emission washes out the effects of the anisotropic mass, and the charge density pattern approaches a Gaussian distribution.

Also evident is an increase in the background noise at high field strength, which we attribute to the noise generated by charge leakage. (See our measurements of the leakage current vs. electric field in $§ 8.3$.) 


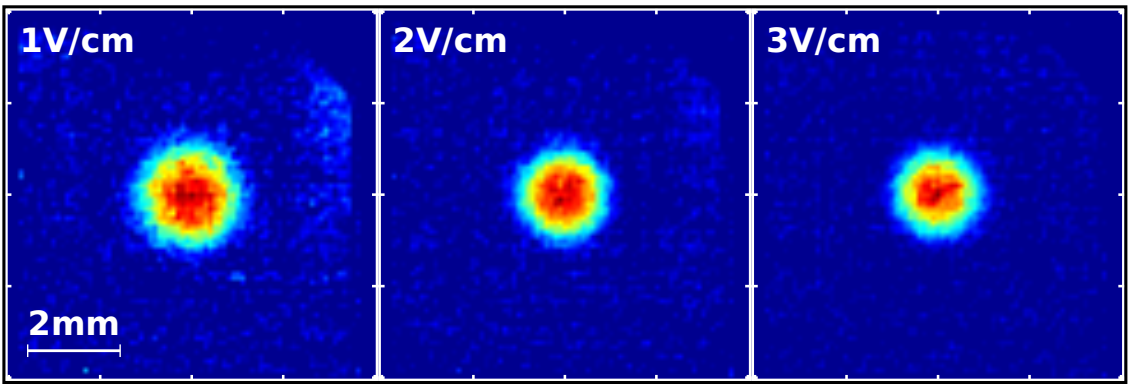

(a) Data

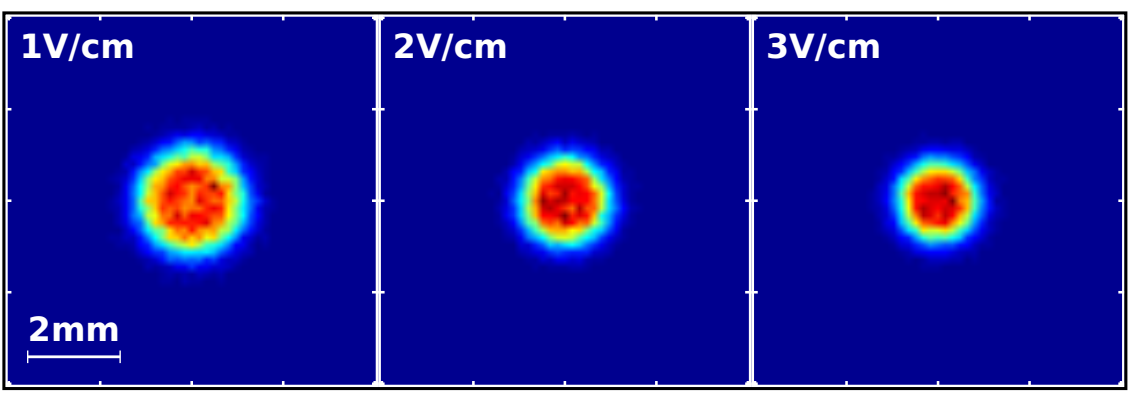

(b) Simulation

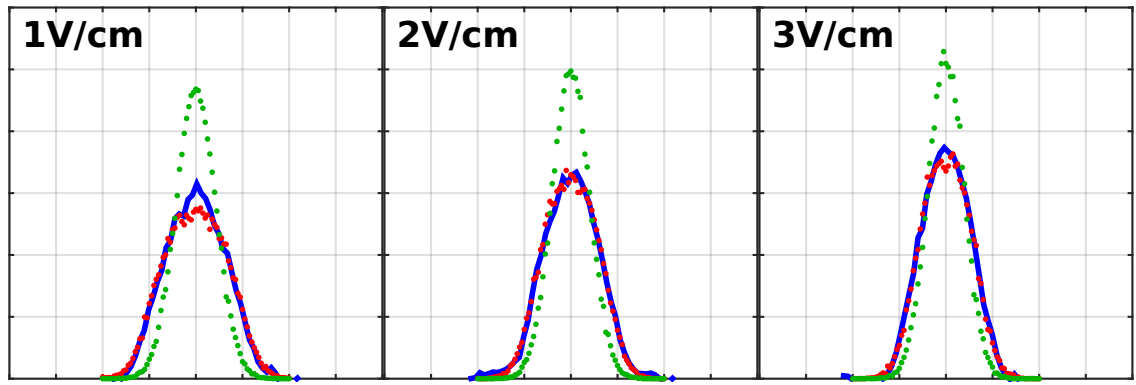

(c) One-Dimensional Projection

Figure 8.1: Hole Charge Density Patterns at 600mK. (a): Data. (b): Simulation. (c): One-dimensional projection of charge density onto x-axis. In (c), the data (solid, blue) are compared to the simulation incorporating electrostatic repulsion (dotted, red), and the simulation with no repulsion (dotted, green). The horizontal scale ranges from $-4 \mathrm{~mm}$ to $+4 \mathrm{~mm}$. The vertical scale is arbitrary. 


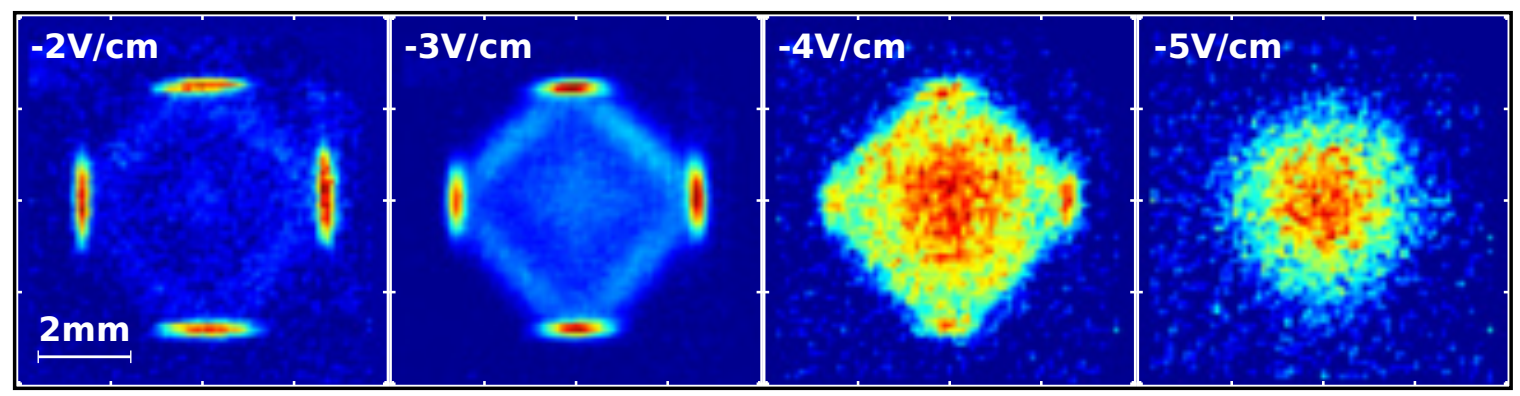

(a) Data

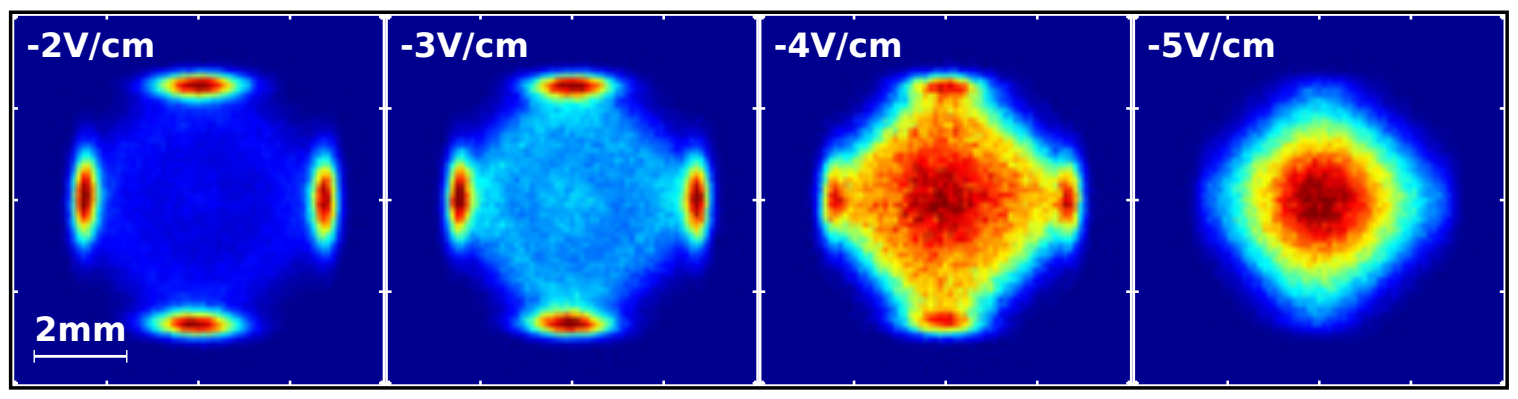

(b) Simulation

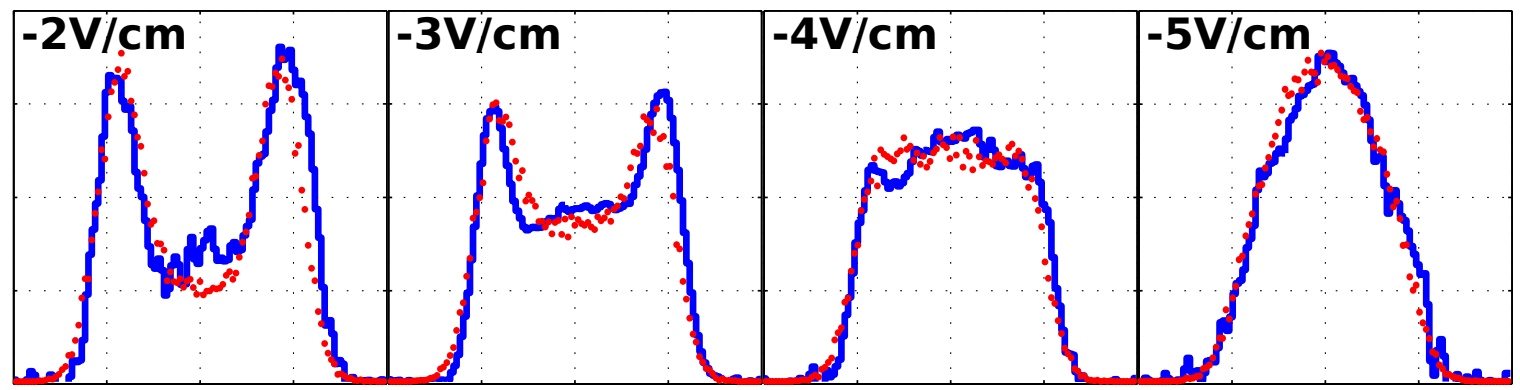

(c) One-Dimensional Diagonal Projection

Figure 8.2: Electron Charge Density Patterns at 600mK. (a): Data. (b): Redl simulation. (c): One-dimensional projection of charge density onto a diagonal axis. In (c), the data (solid, blue) are compared to the Redl simulation employing the HerringVogt approximation (dotted, red). The horizontal scale ranges from $-4 \mathrm{~mm}$ to $+4 \mathrm{~mm}$. The vertical scale is arbitrary. 


\subsection{Inter-Valley Scattering Length}

Figure 8.3 shows the mean path length between electron inter-valley scattering events, projected along the $[0,0,1]$ direction, as a function of electric field strength. The data from our experiment are shown in blue, and are in good agreement with the Redl simulation, shown in green. Note that the parameters which determine the electron scattering rate in the Redl simulation were taken from reference [63], and were not modified to fit our data. For comparison, we also show data from references [64] and [65], plotted on the same axes.

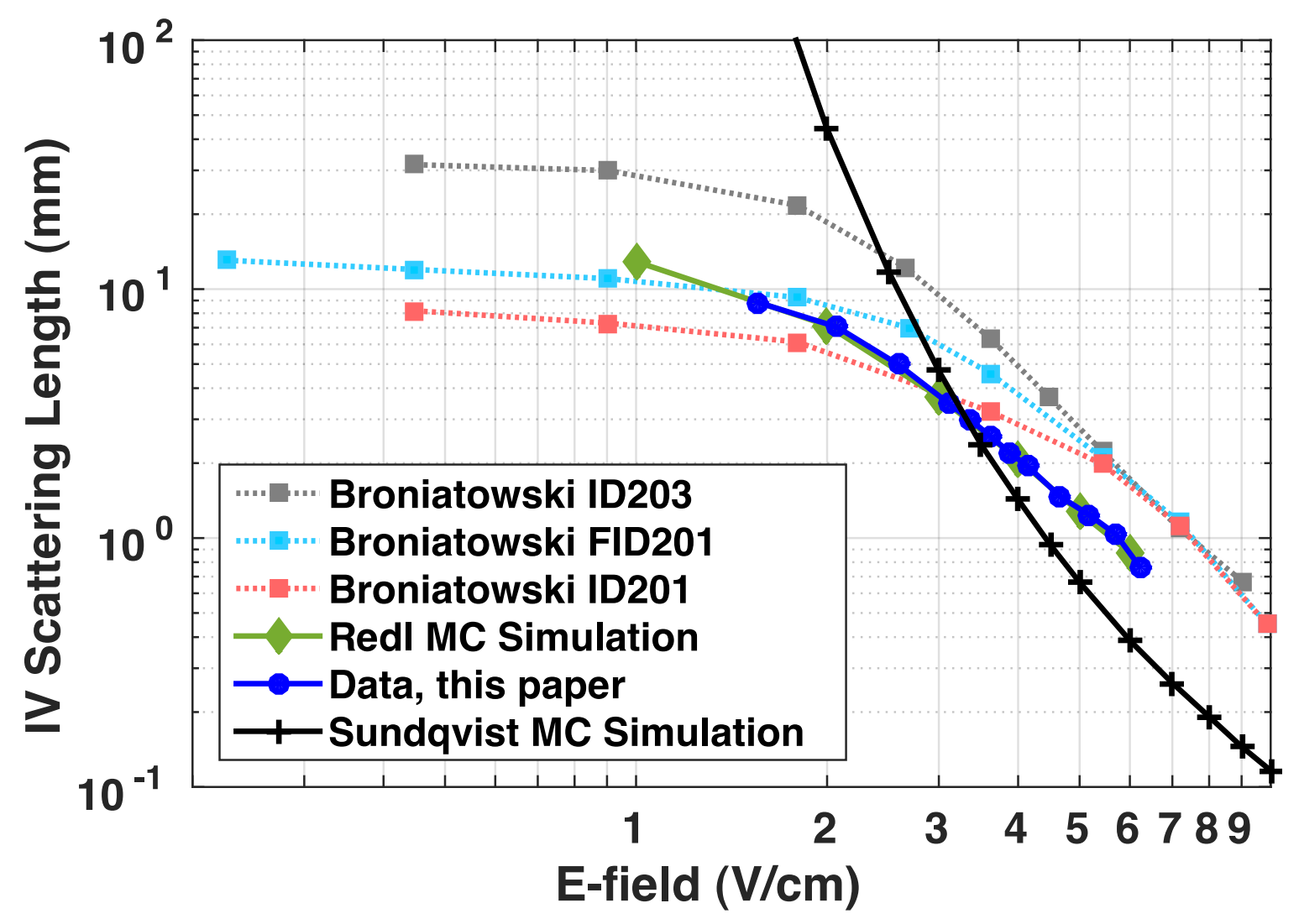

Figure 8.3: Electron inter-valley scattering length at $600 \mathrm{mK}$, projected along $[0,0,1]$, as a function of electric field strength. The data (blue) are compared to the Redl simulation (green) and the Sundqvist simulation (black). Also included is the IV scattering length calculated using data from references [64] and [65]. 


\subsection{Leakage Current and Charge Trapping}

The leakage current was studied by cycling the bias voltage through a loop with exponentially growing amplitude at base temperature $(600 \mathrm{mK}) .{ }^{1}$ For each bias level, voltage was applied and the crystal leakage current was measured with no illumination, followed by a $10 \mathrm{~ms}$ long diffuse flash at zero bias. The leakage current is shown as a function of electric field in the plot on the left in Figure 8.4.

The leakage current has a knee close to a field strength of $4 \mathrm{~V} / \mathrm{cm}$, which is consistent with the higher noise levels observed in the charge density measurements at field strengths above $4 \mathrm{~V} / \mathrm{cm}$. The current also exhibits a notable hysteresis, which may be caused by the trapping of charge in the crystal.

In order to measure the charge trapping as a function of electric field strength, a laser pulse train ${ }^{2}$ was applied to the crystal $^{3}$ at each voltage level. The amplitude of channel 2 was used to estimate the total number of collected charges.

We see from the plot on the right in Figure 8.4 that the charge collection is reduced for electric fields weaker than $1 \mathrm{~V} / \mathrm{cm}$, which is evidence of charge trapping. (More information about charge trapping may be found in reference [4].)
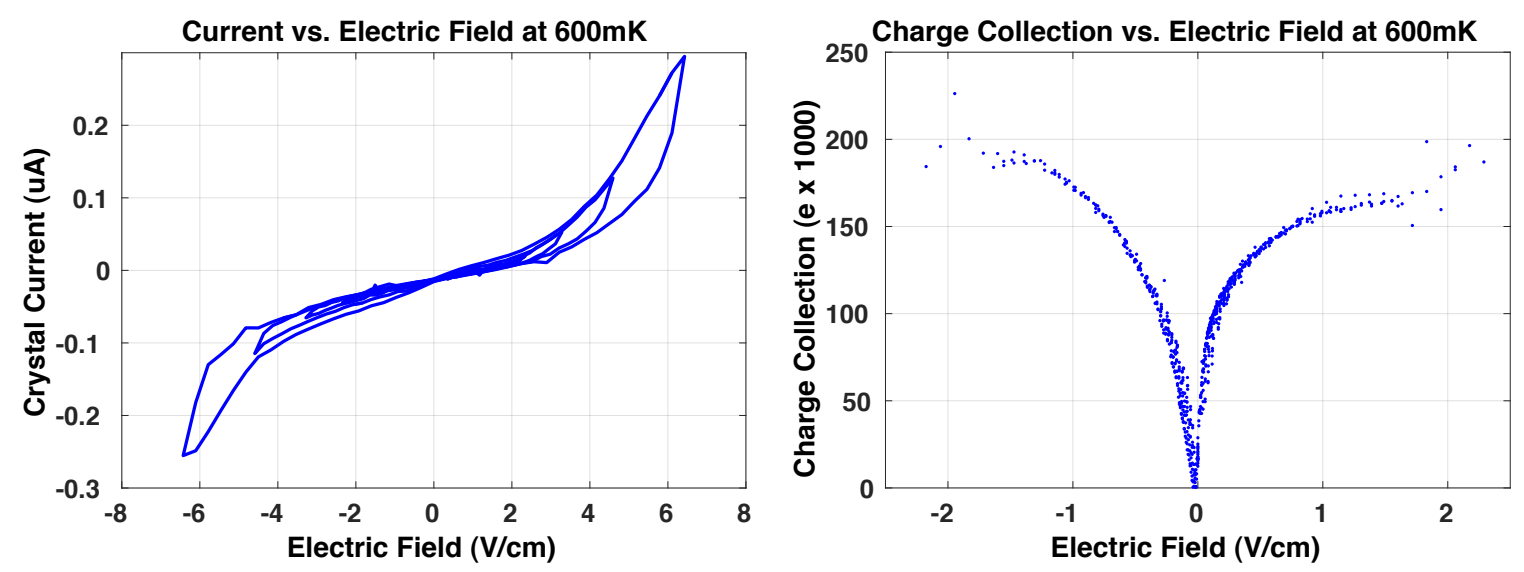

Figure 8.4: Left: current conducted through the crystal as a function of electric field strength (no illumination). Right: charge collection vs. electric field strength.

\footnotetext{
${ }^{1}$ The entire sweep had a duration of about $40 \mathrm{~min}$.

${ }^{2}$ Each pulse had $20 \mu \mathrm{W}$ peak power, $100 \mathrm{~ns}$ duration.

${ }^{3}$ The laser pulses were applied to location $[0.20,0.20]$ in the mirror coordinate system.
} 


\section{Chapter 9}

\section{Discussion}

To obtain good agreement between the Monte Carlo simulation by Peter Redl and the experimental data, several effects need to be taken into account. The first effect is a distortion in the locations of the centers of the four electron clusters. This distortion is explained by a 2 degree offset between the crystal axes and the faces of the crystal, which is consistent with the tolerances of our crystal fabrication process and with subsequent X-ray diffraction measurements of the crystal axes.

The second effect is the lateral spreading of the carriers due to electrostatic repulsion. Without accounting for this effect, our original Monte Carlo simulation for the holes produced charge distributions that were too narrow (see Figure 8.1 (c)). The additional spreading seen in our data can be reproduced in our simulations by incorporating the effect of electrostatic repulsion. Based on the total charge collected by the large electrode on the non-illuminated face, we estimate $1.5 \pm .5 \times 10^{5}$ charge carriers are collected per laser pulse. To obtain good agreement with the data, the simulation contains $2 \pm 1 \times 10^{5}$ holes, assuming the initial charge cloud diameter after the separation of opposite charges is equal in size to the laser spot $(70 \mu \mathrm{m})$.

However, even after accounting for electrostatic repulsion, a discrepancy remains in the case of the electrons, as the electron charge distribution is narrower in the data than in the Redl simulation (compare Figures 8.2 (a) and 8.2 (b)). We attribute this discrepancy to the fact that the Redl simulation uses the Herring-Vogt approximation.[54, 63] As discussed in $\S 5.5$, this approximation provides a faster 
means of simulating the phonon emission process at the expense of violating momentum conservation. This non-conservation of momentum is likely the cause of the extra lateral diffusion seen in the simulated charge density patterns.

To estimate the systematic error introduced by the Herring-Vogt approximation, we use a simulation written by Kyle Sundqvist[4, 66, 67] which explicitly conserves crystal momentum. Figure 9.2 shows a comparison of the Redl simulation (a), with the more accurate, but slower, Sundqvist simulation (b). The widths of the distributions of electrons in a single cluster are shown in Figure 9.3 for both simulations, and for the data.

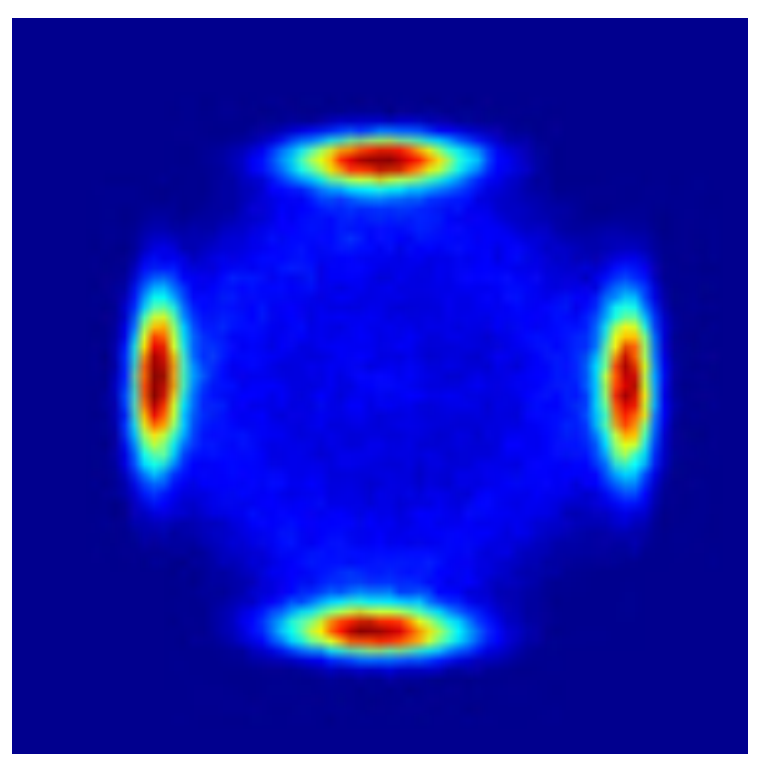

(a) Redl simulation.

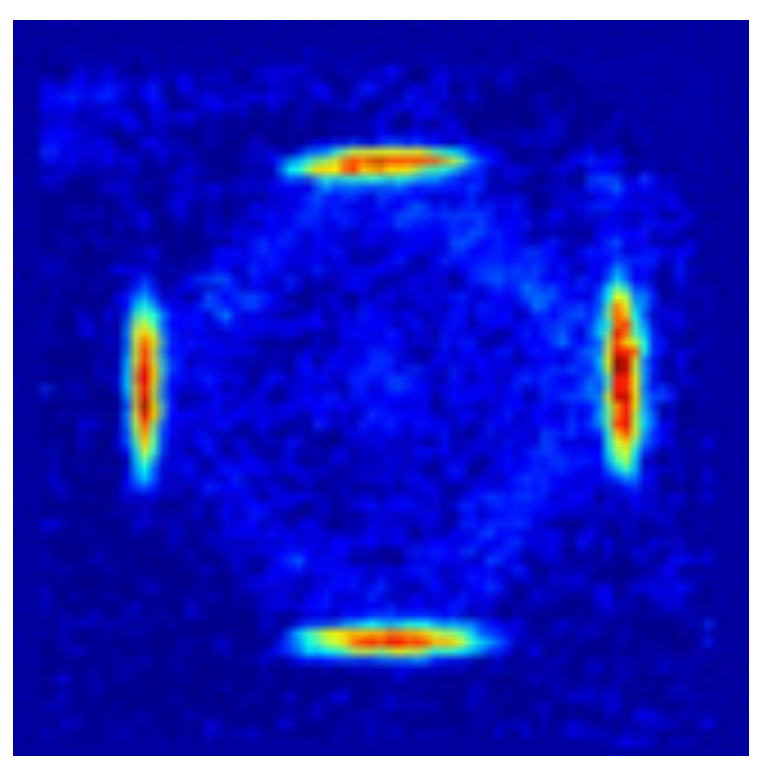

(b) Data.

Figure 9.1: Detailed comparison between data and the Redl simulation for electrons at $2 \mathrm{~V} / \mathrm{cm}$. Note that the electron clusters are wider in the simulation than in the experimental data.

Using the Sundqvist simulation as a standard of comparison, we are able to observe and model the extra lateral spreading of the electrons due to electrostatic repulsion. The repulsion causes the centers of the four electron clusters to spread further from each other, and causes the clusters to spread more in the low-mass directions. Figure 9.2 (c) shows an approximate correction to the Sundqvist simulation for the effects of 


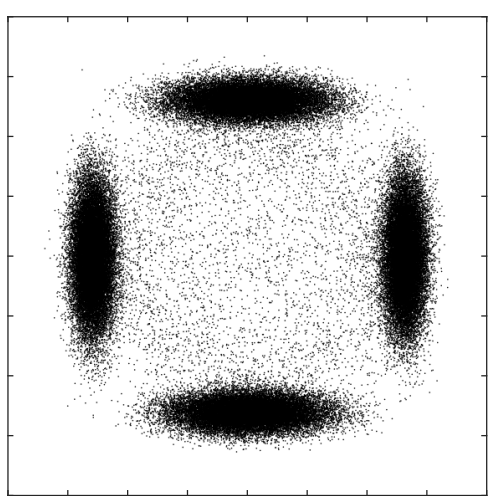

(a) Redl Simulation

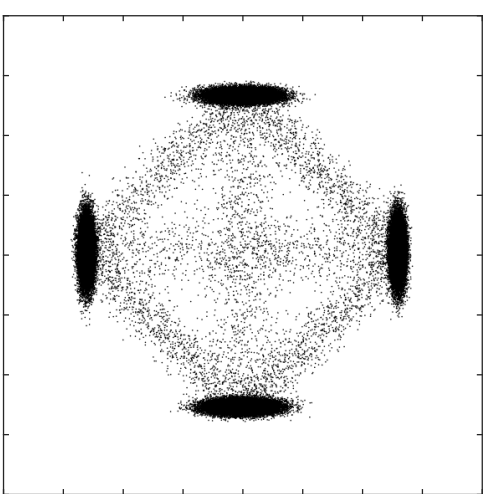

(b) Sundqvist Simulation

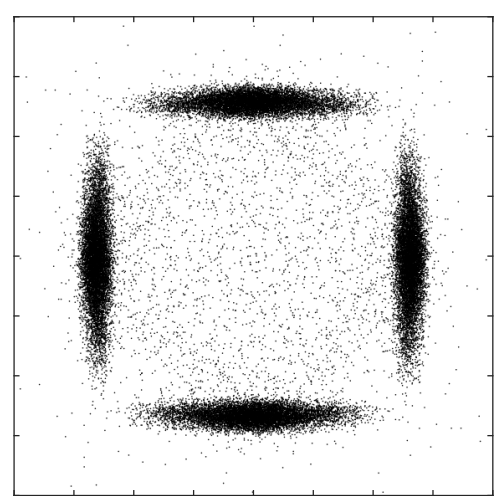

(c) Sundqvist Simulation with Repulsion

Figure 9.2: Comparison of Redl and Sundqvist Simulations: (a), (b), and (c) show scatter plots of the final $\mathrm{x}$ and $\mathrm{y}$ electron locations in three simulations. The axes extend from $-4 \mathrm{~mm}$ to $+4 \mathrm{~mm}$ in the $\mathrm{x}$ and $\mathrm{y}$-directions. (a) Reld simulation using Herring-Vogt approximation, which does not conserve momentum. (b) Sundqvist simulation, which correctly conserves momentum during phonon scattering. $[4,66,67]$ (c) Sundqvist simulation with correction for electrostatic repulsion. For all simulations, the electric field strength was $2 \mathrm{~V} / \mathrm{cm}$, and no impurity scattering was included.

electrostatic repulsion, generated by post-processing the electron trajectories. Figure 9.3 shows how this effect brings the Sundqvist simulation into agreement with the experimental data, assuming the same number of charge-carriers per pulse $\left(2 \pm 1 \times 10^{5}\right)$ as we assume for holes.

We also note that, unlike the Redl simulation, the Sundqvist simulation does not include the effects of impurity scattering. This fact is apparent in Figure 8.3, where the IV scattering length for the Sundqvist simulation diverges for low electric field. However, a discrepancy still exists between the Redl and Sundqvist simulations even at high fields where scattering is dominated by phonon emission. This discrepancy remains unexplained. 

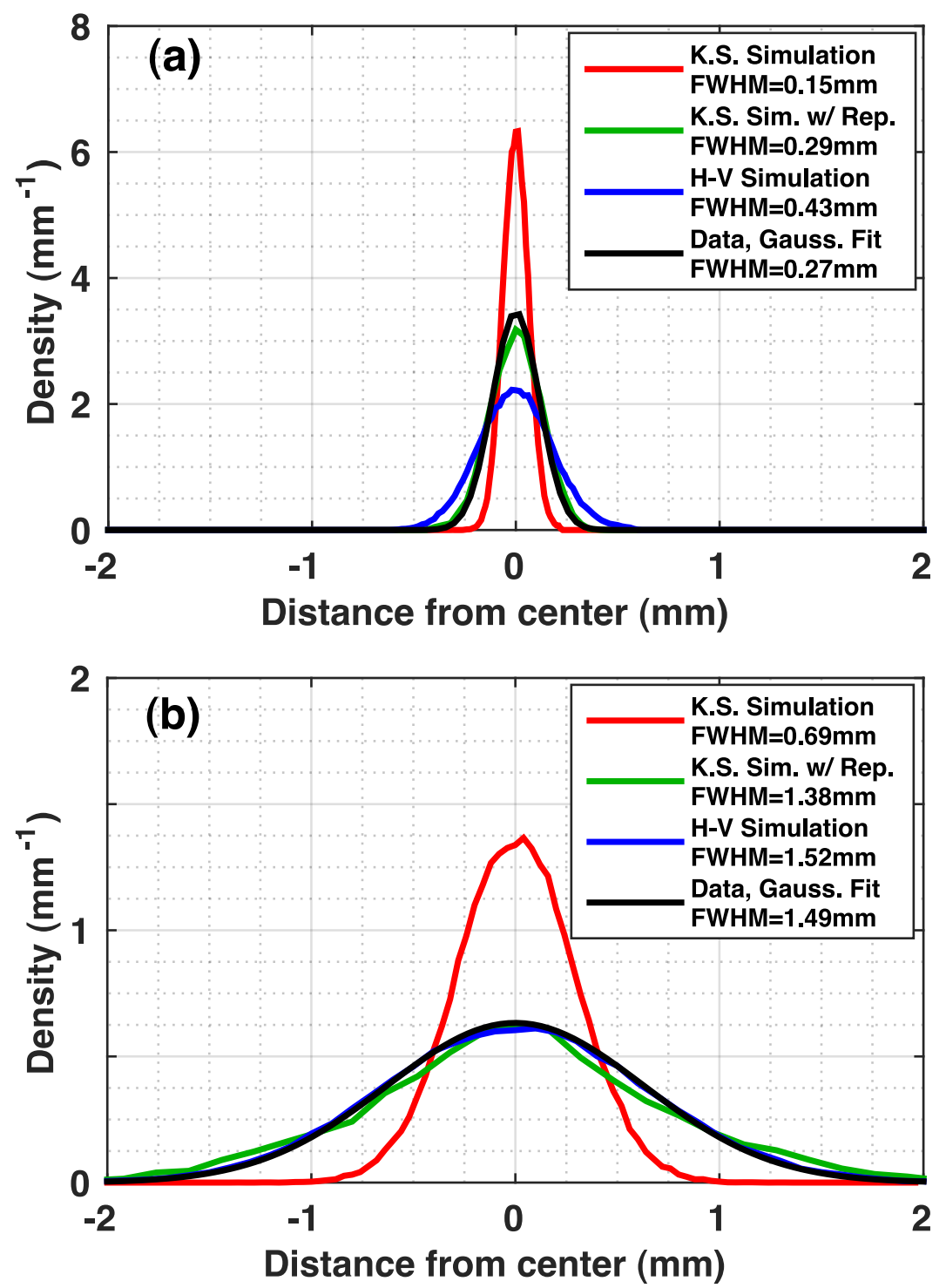

Figure 9.3: Comparison of Lateral Spreading of Electron Charge Density. (a) and (b) show plots of one-dimensional charge density of a single electron cluster, projected along the narrow and wide principle axes, respectively. A Gaussian fit to the data is shown in black, and is compared to three different simulations. For both simulations and data, the electric field strength was $2 \mathrm{~V} / \mathrm{cm}$. 


\section{Chapter 10}

\section{Conclusions and Future Work}

Using the cryogenic scanning apparatus described in this dissertation, we have successfully imaged the two-dimensional charge density distributions of both electrons and holes in high purity germanium crystals at sub-Kelvin temperatures. This work has provided a detailed test of our two Monte Carlo simulations, and our results have revealed the magnitude and relative importance of subtle effects such as electrostatic repulsion between carriers and offsets in the crystal axes. The comparison between experiment and theory indicates the degree of accuracy which our simulations are currently able to achieve, and provides both motivation and direction for further improvements to those simulations.

Because we plan to use silicon detectors in future dark matter searches, we wish to repeat all of the measurements described in this dissertation on high purity silicon samples. The same experimental apparatus can be used for testing silicon, and efforts in this direction are ongoing. In addition to measurements of silicon, we also wish to measure charge propagation along different crystal directions using samples cut from boules grown along different crystal axes. Our future plans include measurement of both silicon and germanium samples cut such that the electric field is oriented along the $[1,0,0]$ and $[1,1,1]$ crystal directions.

Due to recent increased interest in low-mass dark matter $\left(m<10 \mathrm{GeV} / \mathrm{c}^{2}\right)$, the use of high voltage bias is being explored as a means of lowering the minimum energy threshold in our dark matter detectors. For these designs to be practical, the crystals 
must be capable of withstanding high electric fields both in the bulk and and near their surface electrodes. We plan to continue tests of crystal breakdown, such as those described in $\S 8.3$, for crystals with different electrode structures. 


\section{Appendix A}

\section{An Isothermal Cloud}

In this section, a simple model of a dark matter halo is investigated, consisting of a spherically symmetric cloud of particles of mass, $m$, in a state of thermal and hydrostatic equilibrium. Such a model represents an approximation to the final state of a self-gravitating cloud of particles, assuming they have zero net angular momentum and have had sufficient time to come to equilibrium. The orbital rotation curve calculated from this model is shown to closely resemble the shape of observed galactic rotation curves. This illustrates, in a simple way, how the existence of a dark matter halo can explain the flat rotation curves of galaxies.

\section{A.1 Newton's Laws and the Equation of State}

Consider a dark matter halo in hydrostatic and thermal equilibrium which is composed of massive particles with mass, $m$, and temperature, $T$. In the limit where Newtonian gravity applies, the relation between the mass density, $\rho$, and the gravitational potential, $\phi$, is:

$$
\nabla^{2} \phi=-4 \pi G \rho
$$


If the halo is assumed to have spherical symmetry, both the mass density and gravitational potential will be functions of only the radial coordinate, $r$ :

$$
\frac{1}{r^{2}} \frac{d}{d r}\left(r^{2} \frac{d \phi}{d r}\right)=-4 \pi G \rho
$$

In hydrostatic equilibrium, the sum of the pressure force and gravitational force densities must be zero everywhere:

$$
-\nabla p-\rho \nabla \phi=0
$$

where $p$ is the local pressure.

In order to find the equilibrium density profile of the halo, we require an equation of state which relates the local pressure to the local mass density. The simplest assumption is the equation of state of an ideal gas:

$$
p=n k T=\frac{\rho}{m} k T=\rho \frac{2}{3} v_{T}^{2}
$$

where $n$ is the particle number density, $k$ is Boltzmann's constant, and $v_{T}$ is the RMS thermal particle velocity. We may substitute the equation of state into equation A.3 to find a relation between the mass density and the gravitational potential:

$$
\begin{aligned}
-\frac{2}{3} v_{T}^{2} \nabla \rho-\rho \nabla \phi & =0 \\
\nabla\left(\frac{2}{3} v_{T}^{2} \ln \rho+\phi\right) & =0 \\
\frac{2}{3} v_{T}^{2} \ln \rho+\phi & =\mathcal{C}
\end{aligned}
$$

where $\mathcal{C}$ is a constant of integration. 


\section{A.2 A Differential Equation for the Density Pro- file}

The relation between $\phi$ and $\rho$ in equation A.5 may be substituted into equation A.2 to derive a differential equation for $\rho$ :

$$
\frac{2}{3} v_{T}^{2} \frac{1}{r^{2}} \frac{d}{d r}\left(r^{2} \frac{d}{d r} \ln \rho\right)=-4 \pi G \rho
$$

Equation A.6 may be made dimensionless by choosing a characteristic density, $\rho_{0}$, and a characteristic length, $r_{0}$ :

$$
\begin{gathered}
\frac{1}{\xi^{2}} \frac{d}{d \xi}\left(\xi^{2} \frac{d}{d \xi} \ln \delta\right)=-\delta \\
r_{0} \equiv \sqrt{\frac{\frac{2}{3} v_{T}^{2}}{4 \pi G \rho_{0}}} \\
\xi \equiv \frac{r}{r_{0}} \\
\delta \equiv \frac{\rho}{\rho_{0}}
\end{gathered}
$$

This equation for the density profile of a spherically symmetric isothermal halo of gas in hydrostatic equilibrium is known as the Isothermal Lane Emden equation, and was initially studied as an early model for stellar interiors. [68, 69, 70]

\section{A.3 A Simple Solution}

A simple solution to equation A.7 is:

$$
\delta=\frac{2}{\xi^{2}}
$$

or

$$
\rho=\frac{2 \rho_{0} r_{0}^{2}}{r^{2}}=\frac{\frac{2}{3} v_{T}^{2}}{4 \pi G \rho_{0}} \frac{2 \rho_{0}}{r^{2}}=\frac{v_{T}^{2}}{3 \pi G r^{2}}
$$


This solution has the unphysical aspect of a divergent halo mass density at $r=0$. However, note that the total mass enclosed within a radius, $r$, is still finite:

$$
M(r)=\int_{0}^{r} \rho\left(r^{\prime}\right) 4 \pi r^{\prime 2} d r^{\prime}=\int_{0}^{R} \frac{v_{T}^{2}}{3 \pi G r^{\prime 2}} 4 \pi r^{\prime 2} d r=\frac{4 v_{T}^{2} R}{3 G}
$$

The velocity of a body in a circular orbit about the center of the halo at distance, $R$, from the center is:

$$
v(r)=\sqrt{\frac{G M(r)}{r}}=\sqrt{\frac{4}{3} v_{T}^{2}}=\frac{2}{\sqrt{3}} v_{T}
$$

which is independent of the orbital radius. This matches the observed behavior of stars and gas in galaxies at large orbital radii. However, the orbital velocities of stars are observed to approach zero as their distances from the galactic center approach zero. In order to better model this behavior, we must more accurately treat the halo density at the point $r=0$.

\section{A.4 A Solution with Realistic Boundary Condi- tions}

Let us assume that the halo mass density is both finite and smooth at $r=0$. Let $\rho_{0}$ represent the mass density at $r=0$. The corresponding boundary conditions on $\delta(\xi)$ at $\xi=0$ are:

$$
\begin{aligned}
\delta(0) & =1 \\
\delta^{\prime}(0) & =0
\end{aligned}
$$

Because equation A.7 is a second order differential equation, these two boundary conditions uniquely determine its solution. The solution, computed numerically, is plotted in Figure A.1.

The total mass enclosed within radius, $r$, may be found by integration of $\delta(\xi)$ :

$$
M(r)=\int_{0}^{r} \rho\left(r^{\prime}\right) 4 \pi r^{\prime 2} d r^{\prime}=\rho_{0} r_{0}^{3} \int_{0}^{r / r_{0}} \delta(\xi) 4 \pi \xi^{2} d \xi
$$




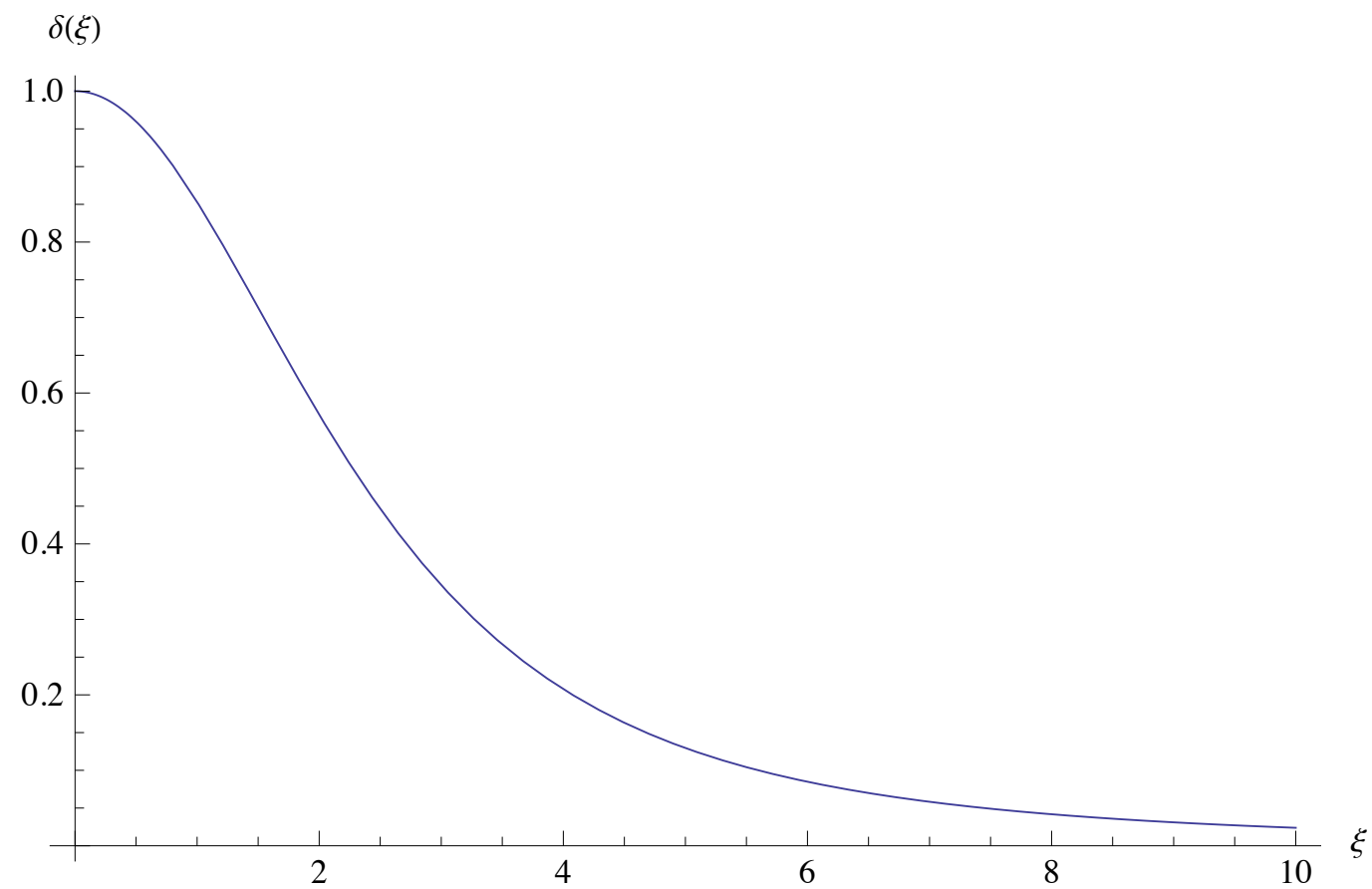

Figure A.1: Dimensionless halo mass density, $\delta$, vs. dimensionless radius, $\xi$.

We may define a characteristic mass scale:

$$
m_{0} \equiv \rho_{0} r_{0}^{3}
$$

Let us define the dimensionless cumulative mass function, $\mu(\xi)$, to be:

$$
\mu(\xi) \equiv \frac{M\left(\xi r_{0}\right)}{m_{0}}=\int_{0}^{\xi} \delta\left(\xi^{\prime}\right) 4 \pi \xi^{\prime 2} d \xi^{\prime}
$$

which is determined by the dimensionless density profile, $\delta(\xi)$, alone. The dimensionless cumulative mass function is plotted in Figure A.2.

The velocity of a body in circular orbit about the center of the halo at a dimensionless distance, $\xi$, from the center is:

$$
v(\xi)=\sqrt{\frac{G M\left(\xi r_{0}\right)}{\xi r_{0}}}=\sqrt{\frac{G \rho_{0} r_{0}^{3} \mu(\xi)}{\xi r_{0}}}=\sqrt{\frac{v_{T}^{2}}{6 \pi} \frac{\mu(\xi)}{\xi}}
$$




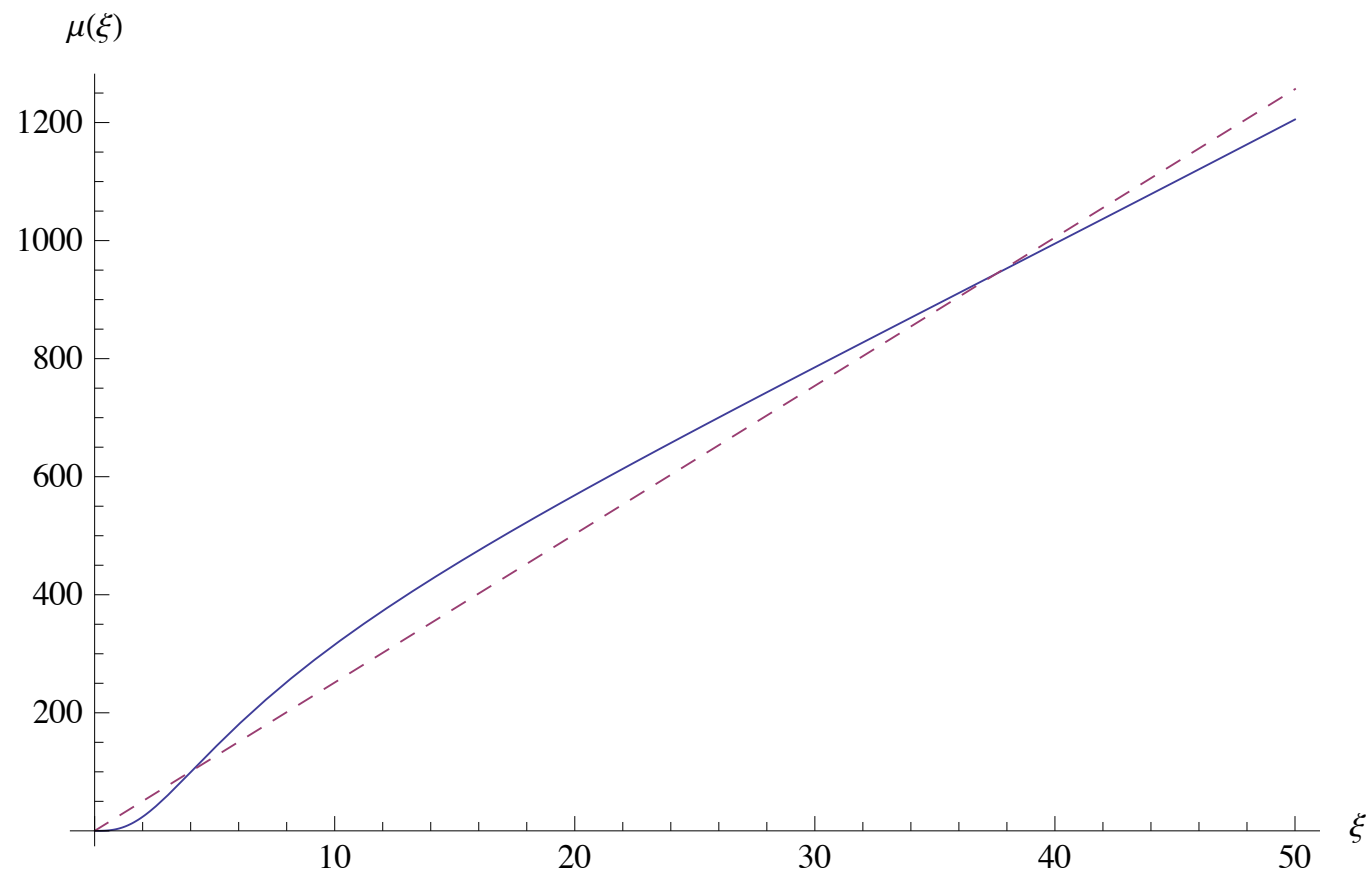

Figure A.2: Dimensionless cumulative mass, $\mu$, vs. dimensionless radius, $\xi$. The dashed line represents $\mu=8 \pi \xi$.

Based on the solution from $§$ A.3, we may define a characteristic velocity scale:

$$
v_{0} \equiv \frac{2}{\sqrt{3}} v_{T}
$$

Let us define the dimensionless orbital velocity, $u(\xi)$, to be:

$$
u(\xi) \equiv \frac{v(\xi)}{v_{0}}=\frac{v(\xi)}{\sqrt{\frac{4}{3} v_{T}^{2}}}=\sqrt{\frac{\mu(\xi)}{8 \pi \xi}}
$$

The dimensionless orbital velocity, $u(\xi)$, depends only on the dimensionless cumulative mass function, $\mu(\xi)$, and is plotted in Figure A.3. As can be seen in Figure A.3, $u(\xi)$ approaches a value of 1 as the orbital radius approaches infinity. 


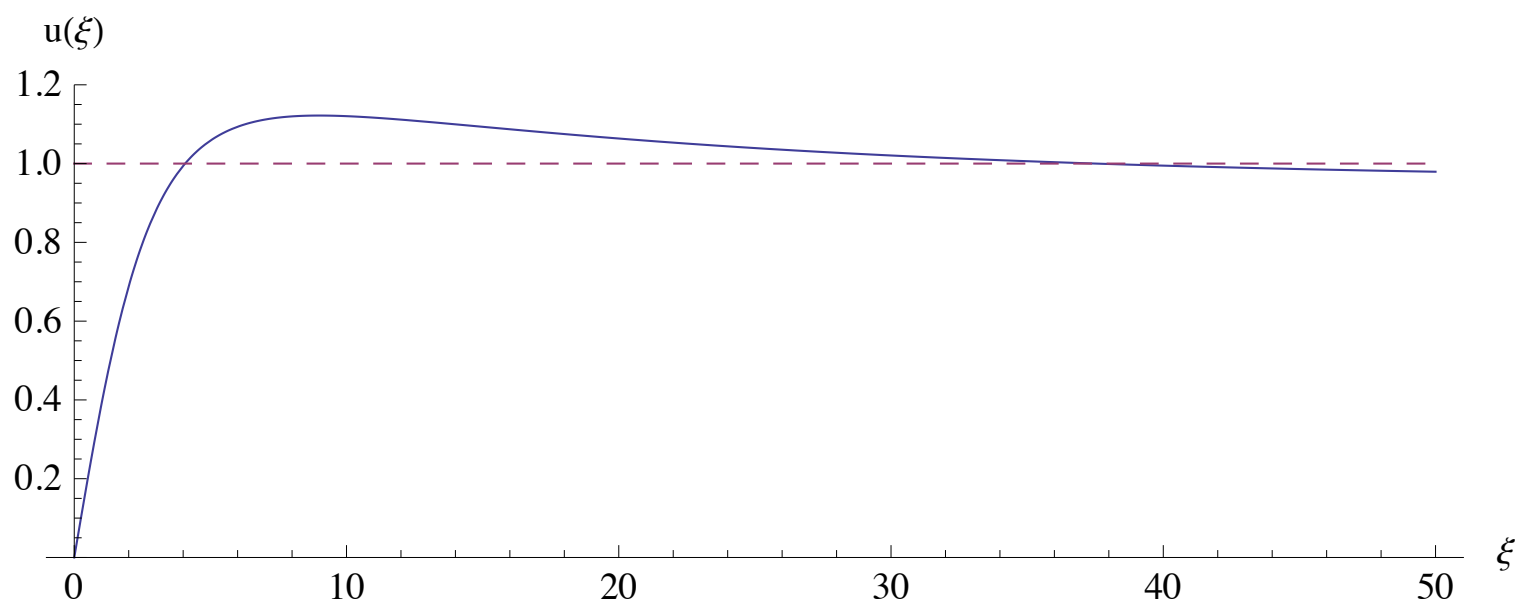

Figure A.3: Dimensionless rotation curve.

\section{A.5 Comparison to Observation}

Figure A.4 shows the comparison between the observed rotation curves of 31 galaxies, using data from reference [71], and the theoretical rotation curve of a spherically symmetric halo in thermal and hydrostatic equilibrium. We see that, despite the simplicity of our model, it still captures most of the prominent features of the galactic rotation curves.

This calculation provides motivation for the idea that a dark matter halo is capable of explaining the flat rotation curves observed in galaxies. Although more complex numerical simulations have provided better estimates for the shapes of dark matter halo density profiles, it is useful to see how the observed rotation curves can be reproduced in a natural way from a simple model without the necessity of any ad hoc assumptions. 


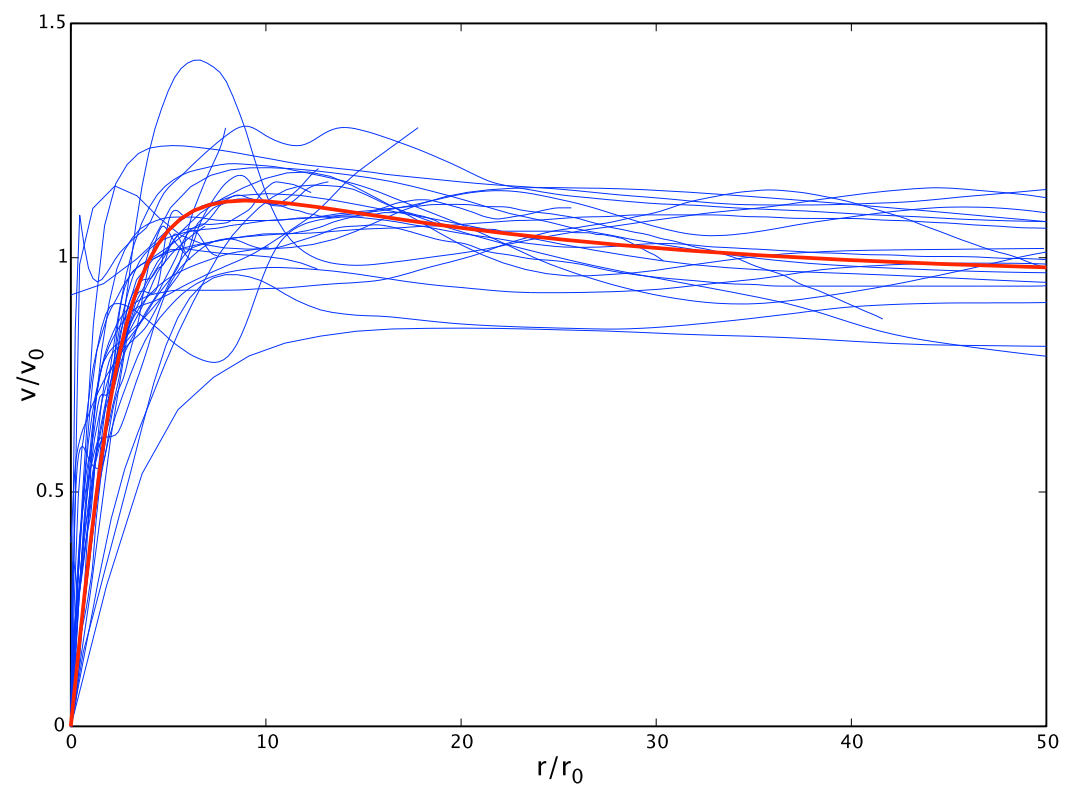

Figure A.4: Experimentally measured rotation curves from 31 galaxies (blue) scaled to fit the theoretical curve from Figure A.3 (red) by the method of least squares. Data from reference [71].

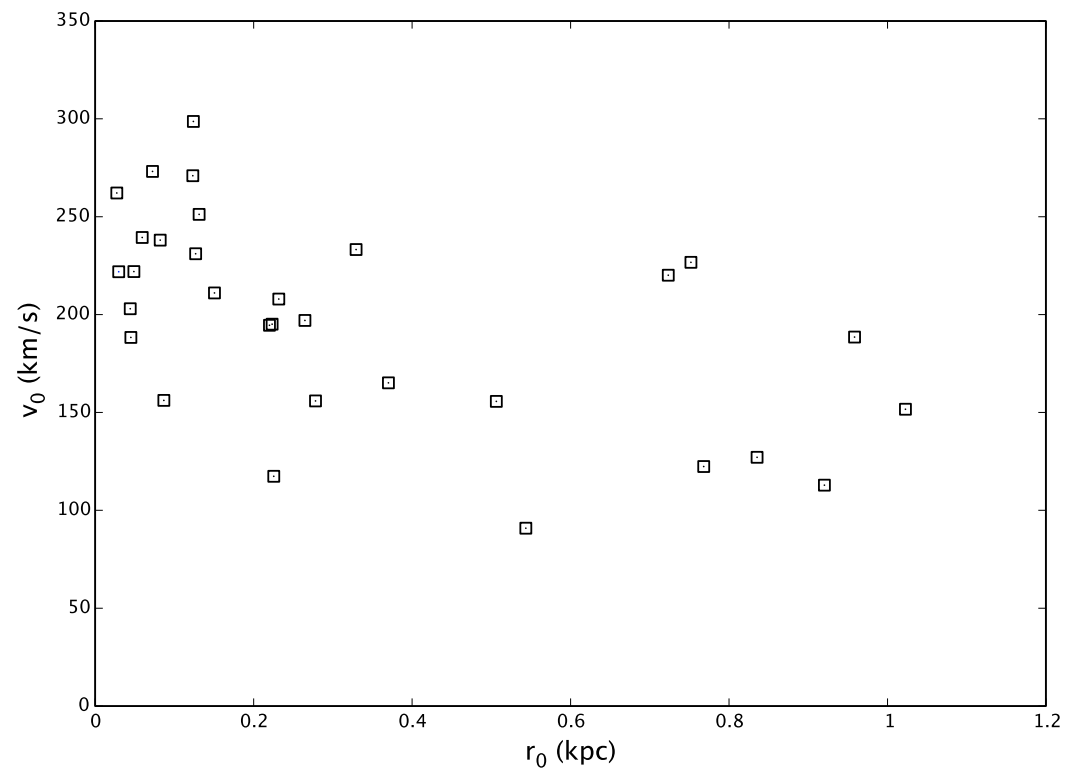

Figure A.5: Best fit values of orbital velocity, $v_{0}$, and core radius, $r_{0}$, for the 31 galaxies whose scaled rotation curves are plotted in Figure A.4. 


\section{Appendix B}

\section{WIMP Scattering Cross-Section}

In this section, the scattering cross-section is found between a Weakly Interacting Massive Particle (WIMP) and a free particle of ordinary matter, assuming the particle of ordinary matter is initially at rest, the WIMP speed is much slower than the speed of light, and the scattering is mediated by an electrically neutral vector boson.

\section{B.1 Kinematics}

Assume a WIMP with a mass, $m_{\chi}$, traveling at velocity, $\mathbf{v}_{\chi}$, impacts a free fermionic particle of ordinary matter of mass, $m_{f}$, initially at rest. Let the momentum of the particle of ordinary matter after the collision be denoted by $\mathbf{q}$. In the non-relativistic limit, the total initial kinetic energy, $K$, is:

$$
K=\frac{1}{2} m_{\chi} \mathbf{v}_{\chi}^{2}
$$

Assuming momentum conservation, the total final kinetic energy, $K^{\prime}$, is:

$$
\begin{aligned}
K^{\prime} & =\frac{\mathbf{q}^{2}}{2 m_{f}}+\frac{\left(m_{\chi} \mathbf{v}_{\chi}-\mathbf{q}\right)^{2}}{2 m_{\chi}} \\
& =\frac{\mathbf{q}^{2}}{2 m_{f}}+\frac{1}{2} m_{\chi} \mathbf{v}_{\chi}^{2}-\mathbf{v}_{\chi} \cdot \mathbf{q}+\frac{\mathbf{q}^{2}}{2 m_{\chi}}
\end{aligned}
$$




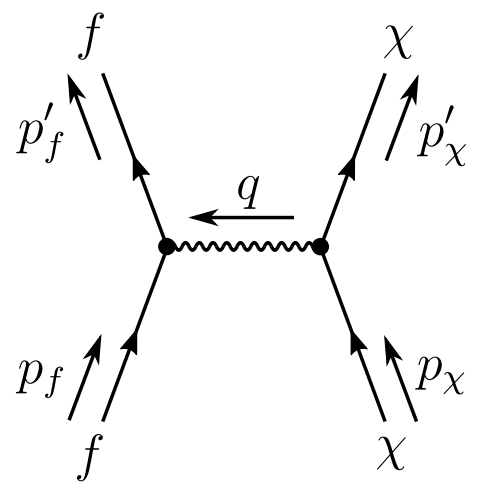

Figure B.1: Feynmann diagram for the scattering of a WIMP $(\chi)$ from a fermion $(f)$, through the exchange of a massive vector boson.

The difference between the final and initial kinetic energies is:

$$
K^{\prime}-K=\frac{\mathbf{q}^{2}}{2 \mu}-\mathbf{v}_{\chi} \cdot \mathbf{q}
$$

where $\mu$ is the reduced mass of the pair of particles:

$$
\mu \equiv \frac{1}{\frac{1}{m_{f}}+\frac{1}{m_{\chi}}}
$$

In the case of an elastic collision, kinetic energy is conserved and $K^{\prime}=K$. However, the expression for $K^{\prime}-K$ as a function of $\mathbf{q}$ will become important later in the calculation of the scattering cross-section.

\section{B.2 Scattering Amplitude}

Consider the case in which the WIMP, denoted by $\chi$, and the fermion, denoted by $f$, interact through the exchange of a vector boson, denoted by $B^{\mu}$, which has mass $m_{B}$. (See Figure B.1.) In the case where $q$ is much smaller than $m_{B}$, the boson propagator 
can be approximated as: ${ }^{1}$

$$
\left\langle B^{\mu}(q) B^{\nu}(-q)\right\rangle=\frac{-i g^{\mu \nu}}{q^{2}-m_{B}^{2}} \approx \frac{i g^{\mu \nu}}{m_{B}^{2}}
$$

where $g^{\mu \nu}$ is the space-time metric. Using this approximation, the effective Lagrangian for the interaction is: ${ }^{2}$

$$
\Delta \mathcal{L}=\frac{g^{2}}{m_{B}^{2}}\left(\bar{f} \gamma^{\mu}\left(\alpha_{f}+\beta_{f} \gamma^{5}\right) f\right)\left(\bar{\chi} \gamma_{\mu}\left(\alpha_{\chi}+\beta_{\chi} \gamma^{5}\right) \chi\right)
$$

where $g$ is the coupling coefficient for the $B$ field and where the $\alpha$ and $\beta$ factors represent spin-dependent coupling coefficients.

It may safely be assumed that the incoming particles are not spin polarized. Therefore, we are interested in the square of the matrix element, $|\mathcal{M}|^{2}$, averaged over all initial spin states and summed over all final spin states. The result contains dot products between all combinations of the four-momentum vectors of the interacting particles. $^{3}$ In the non-relativistic limit, we may approximate such dot products by the product of the magnitudes of the two vectors, which are equal to $m_{f}$ and $m_{\chi}$ for the fermion and WIMP, respectively. Using this approximation, the spin-averaged squared matrix element, $|\mathcal{M}|^{2}$, is:

$$
\frac{1}{4} \sum_{\text {spins }}|\mathcal{M}|^{2}=\frac{g^{\prime 4}}{m_{B}^{4}} m_{f}^{2} m_{\chi}^{2}
$$

where $g^{\prime}$ is an effective coupling coefficient which depends on the $\alpha$ and $\beta$ coefficients.

\footnotetext{
${ }^{1}$ This approximation was first employed by Enrico Fermi in 1933 in the context of beta decay. See reference [72], pg. 708.

${ }^{2}$ See reference [72], pg. 709.

${ }^{3}$ See, for example, reference [72], pg. 154.
} 


\section{B.3 Scattering Cross-Section}

The differential cross-section for an interaction between two particles, $A$, and $B$, is: ${ }^{4}$

$$
\begin{aligned}
d \sigma= & \frac{1}{2 \mathscr{E}_{A} 2 \mathscr{E}_{B}\left|\mathbf{v}_{A}-\mathbf{v}_{B}\right|}\left(\prod_{i} \frac{d^{3} \mathbf{p}_{i}}{(2 \pi)^{3}} \frac{1}{2 \mathscr{E}_{i}}\right) \\
& \times \mid \mathcal{M}\left(p_{A},\left.p_{B} \rightarrow\left\{p_{i}\right\}\right|^{2}(2 \pi)^{4} \delta^{(4)}\left(p_{A}+p_{B}-\sum p_{i}\right)\right.
\end{aligned}
$$

where $\mathscr{E}_{X}$ represents the relativistic energy of particle $X$, and where the set, $\left\{p_{i}\right\}$, represents the momentum states of the final particles produced by the interaction. If we are interested in the total cross-section, we must integrate the differential crosssection, $d \sigma$, over the phase space of all final states.

For an elastic collision between a WIMP and a fermion, the total interaction cross-section, $\sigma$, is:

$$
\begin{aligned}
\sigma & =\frac{1}{16 \mathscr{E}_{f} \mathscr{E}_{\chi}\left|\mathbf{v}_{\chi}\right|(2 \pi)^{2}} \int \frac{d^{3} \mathbf{p}_{f}^{\prime}}{\mathscr{E}_{f}^{\prime}} \frac{d^{3} \mathbf{p}_{\chi}^{\prime}}{\mathscr{E}_{\chi}^{\prime}}\left(\frac{1}{4} \sum_{\mathrm{spins}}|\mathcal{M}|^{2}\right) \delta^{(4)}\left(p_{f}^{\prime}+p_{\chi}^{\prime}-p_{f}-p_{\chi}\right) \\
& =\frac{1}{64 \pi^{2}\left|\mathbf{v}_{\chi}\right| m_{f}^{2} m_{\chi}^{2}} \int d^{3} \mathbf{p}_{f}\left(\frac{g^{\prime 4}}{m_{B}^{4}} m_{f}^{2} m_{\chi}^{2}\right) \delta\left(\mathscr{E}_{f}^{\prime}+\mathscr{E}_{\chi}^{\prime}-\mathscr{E}_{f}-\mathscr{E}_{\chi}\right) \\
& =\frac{g^{4}}{64 \pi^{2}\left|\mathbf{v}_{\chi}\right| m_{B}^{4}} \int d^{3} \mathbf{p}_{f} \delta\left(K^{\prime}-K\right) \\
& =\frac{g^{\prime 4}}{64 \pi^{2}\left|\mathbf{v}_{\chi}\right| m_{B}^{4}} \int d^{3} \mathbf{q} \delta\left(\frac{\mathbf{q}^{2}}{2 \mu}-\mathbf{v}_{\chi} \cdot \mathbf{q}\right) \\
& =\frac{g^{4}}{64 \pi^{2}\left|\mathbf{v}_{\chi}\right| m_{B}^{4}} \int_{0}^{\infty} d q_{\perp} \int_{-\infty}^{\infty} d q_{\|} 2 \pi q_{\perp} \delta\left(\frac{q_{\perp}^{2}+q_{\|}^{2}}{2 \mu}-\left|\mathbf{v}_{\chi}\right| q_{\|}\right) \\
& =\frac{g^{\prime 4}}{64 \pi^{2}\left|\mathbf{v}_{\chi}\right| m_{B}^{4}} \int_{0}^{\infty} d q_{\perp} \Theta\left(\mu\left|\mathbf{v}_{\chi}\right|-q_{\perp}\right) \frac{4 \pi \mu q_{\perp}}{\sqrt{\mu^{2}\left|\mathbf{v}_{\chi}\right|^{2}-q_{\perp}^{2}}} \\
& =\frac{g^{\prime 4}}{64 \pi^{2}\left|\mathbf{v}_{\chi}\right| m_{B}^{4}} 4 \pi \mu^{2}\left|\mathbf{v}_{\chi}\right| \\
& =\frac{g^{\prime 4}}{16 \pi} \frac{\mu^{2}}{m_{B}^{4}}
\end{aligned}
$$

\footnotetext{
${ }^{4}$ See reference [72], pg. 106.
} 
Therefore, we see that the total cross-section is proportional to the square of the reduced mass, and inversely proportional to the fourth power of the mass of the vector boson.

\section{B.4 Composite Particles}

In the limit of low momentum transfer between a WIMP and a composite particle, the scattered waves from each component of the composite particle will add coherently. If each amplitude is identical in magnitude and phase, then the total scattering amplitude will be directly proportional to the number of component particles, $A$. The scattering probability and scattering cross-section are proportional to the square of this scattering amplitude. Therefore, in the case of a composite particle with $A$ identical constituents, the scattering cross-section will grow proportionally to $A^{2}$.

Two effects may cause partial cancellations in the total scattering amplitude. The first is the relative phase shifts of the constituents caused by the spatial extent of the composite particle, which become important when the size of the composite particle is large compared to the wavelength of the momentum transfer. This factor is called the form factor of the composite particle, and can be thought of as a spatial Fourier transform of the internal particle density of the composite particle.[21]

The second effect occurs when the composite particle consists of constituents which do not have identical scattering amplitudes. Such may be the case, for example, if the scattering amplitude depends on spin. See reference [28] for a detailed overview of spin-dependent interactions.

\section{B.5 Bound Particles}

The phase-space integrals performed in $\S$ B.3 are based on the assumption that the fermion and WIMP were both free particles. Situations exist in which this is a poor approximation, such as the case of electrons in a solid. See references [73] and [74] for analyses of low-mass WIMP interactions with bound electrons. 


\section{Appendix $\mathrm{C}$}

\section{Quasiballistic Diffusion of Phonons}

In this section, a first-order linear differential equation is derived for the phase-space density of phonons moving through a medium in the limit where both diffusive and ballistic transport effects are of equal importance. This differential equation can be cast in a matrix form, and is amenable to numerical solution using standard eigenvalue/eigenvector methods. The differential equation for particle density is demonstrated to reduce to the diffusion equation in the limit where the spatial variations are much larger than the mean scattering length and the temporal variations are much longer than the mean scattering time. (See [75] for a similar approach in one dimension.)

\section{C.1 The Ballistic Transport of Particles}

Consider an isotropic and homogeneous $D$-dimensional medium which contains a collection of particles, each of which obeys the following Hamiltonian:

$$
\mathscr{H}(q, p)=c|p|
$$

The equations of motion for any one of these particles will be:

$$
\begin{aligned}
& \dot{p}=-\partial_{q} \mathscr{H}=0 \\
& \dot{q}=\partial_{p} \mathscr{H}=c \hat{p}
\end{aligned}
$$


where $\hat{p}$ denotes a unit vector which points in the same direction as the vector $p$.

Now consider a large ensemble of these particles. The particles may each be described by a point in a $2 D$-dimensional single-particle phase space. Because there are no interactions between particles, the particles will evolve in time independently according to the equations of motion above.

Let the continuous function $f(q, p)$ describe the density of these points in phase space in the limit where the number of particles is very large, such that the number of particles in a given volume element is very close to $f(q, p) d^{D} q d^{D} p$.

The flow of points in phase space may be described by a vector field, $v$, defined such that its first $D$ components are equal to $\dot{q}$, and its second $D$ components are equal to $\dot{p}$. The current density, $j$, of points in phase space is given by:

$$
j(q, p)=f(q, p) v(q, p)
$$

If it is assumed that the number of particles is locally conserved, the rate of change of $f$ must be equal to minus the divergence of the current density:

$$
\partial_{t} f=-\nabla \cdot j=-v \cdot \nabla f-f \nabla \cdot v
$$

From this, it follows that the convective derivative of $f$ is directly proportional to the divergence of $v$ :

$$
D_{t} f \equiv \partial_{t} f+v \cdot \nabla f=-f \nabla \cdot v
$$

However, Liouville's Theorem states that $\nabla \cdot v=0$ :

$$
\nabla \cdot v=\partial_{q} \cdot \dot{q}+\partial_{p} \cdot \dot{p}=\partial_{q} \cdot \partial_{p} \mathscr{H}-\partial_{p} \cdot \partial_{q} \mathscr{H}=0
$$

Therefore, in the case of ballistic transport, the convective derivative of $f$ is zero, and it will obey the following linear, first-order differential equation:

$$
\dot{f}(q, p)=-v \cdot \nabla f(q, p)=-c \hat{p} \cdot \partial_{q} f(q, p)
$$

Note that because the Hamiltonian is time-independent, it will be a conserved 
quantity in the motion of any one particle, which implies that for each particle the vector, $p$, has a constant magnitude.

\section{C.2 Elastic Scattering}

In the process of elastic scattering, let it be assumed that a particle will change only the direction of its momentum vector, $p$. The magnitude of its momentum, $|p|$, and the position, $q$, will both remain unchanged. Let the time, $\tau$, represent the average time between scattering events, which are assumed to occur for each particle in a Poisson process.

Because the magnitude of $p$ is unchanged either by elastic scattering or by ballistic transport, and because the velocity of a particle, $\dot{q}$, depends only on the direction of $p$, and not on its magnitude, then, without loss of generality, the behavior of a collection of particles undergoing elastic scattering and ballistic transport may be described by a density function which is independent of the magnitude of $p$.

Let this new density operator, $\phi(q, \hat{p})$, be defined in the following way:

$$
\phi(q, \hat{p}) \equiv \int_{0}^{\infty} d|p||p|^{D} f(q,|p| \hat{p})
$$

According to this density operator, the number of particles, $N$, in a small volume, $d^{D} q$, around point $q$, traveling in a group of directions within a certain solid angle, $d \Omega$, around the direction $\hat{p}$, is: $N=d^{D} q d \Omega \phi(q, \hat{p})$.

Consider a region $d^{D} q$ centered around point $q$. The number of particles in this region is:

$$
N=d^{D} q \int d \Omega \phi(q, \hat{p})
$$

The total rate of scattering, $r_{\mathrm{s}}$, occurring in this region is:

$$
r_{\mathrm{s}}=\frac{N}{\tau}
$$

Now consider a single bin of solid angle, $d \Omega$. The number of particles contained in 
this bin is:

$$
M=d^{D} q d \Omega \phi(q, \hat{p})
$$

Let the scattering process be assumed to be isotropic, such that a particle scatters from any direction, to any direction, with equal probability. The average rate at which particles are lost, $r_{\text {loss }}$, from the small bin is:

$$
r_{\text {loss }}=\frac{M}{\tau}
$$

The average rate at which particles are gained in this bin is:

$$
r_{\text {gain }}=\frac{d \Omega}{\mathcal{S}_{D-1}} r_{\mathrm{s}}=\frac{d \Omega}{\mathcal{S}_{D-1}} \frac{N}{\tau}
$$

where $\mathcal{S}_{n}$ denotes the surface area of a unit $n$-sphere. Combining these two equations, we have the relations:

$$
\begin{aligned}
\dot{M} & =-r_{\text {loss }}+r_{\text {gain }}=-\frac{M}{\tau}+\frac{d \Omega}{\mathcal{S}_{D-1}} \frac{N}{\tau} \\
d^{D} q d \Omega \dot{\phi}(q, \hat{p}) & =-\frac{1}{\tau} d^{D} q d \Omega \phi(q, \hat{p})+\frac{d \Omega}{\mathcal{S}_{D-1}} \frac{1}{\tau} d^{D} q \int d \Omega \phi(q, \hat{p}) \\
\dot{\phi}(q, \hat{p}) & =-\frac{1}{\tau} \phi(q, \hat{p})+\frac{1}{\tau} \int \frac{d \Omega}{\mathcal{S}_{D-1}} \phi(q, \hat{p}) \\
\dot{\phi}(q, \hat{p}) & =-\frac{1}{\tau}(\phi(q, \hat{p})-\bar{\phi}(q))
\end{aligned}
$$

where $\bar{\phi}(q)$ is defined to be the average of $\phi(q, \hat{p})$ over all directions:

$$
\bar{\phi}(q) \equiv \int \frac{d \Omega}{\mathcal{S}_{D-1}} \phi(q, \hat{p})
$$

\section{C.3 Time Evolution}

The time evolution of the density function, $\phi(q, \hat{p})$, including both elastic scattering and ballistic transport is:

$$
\dot{\phi}(q, \hat{p})=-c \hat{p} \cdot \partial_{q} \phi(q, \hat{p})-\frac{1}{\tau}(\phi(q, \hat{p})-\bar{\phi}(q))
$$


This equation has the form:

$$
\dot{\phi}(t)=H \phi(t)
$$

where $H$ is a linear operator acting on the function $\phi$. This is a linear, first-order differential equation for $\phi$, which has the formal solution:

$$
\phi(t)=e^{H t} \phi(0)
$$

This equation may be solved numerically by dividing the position and angular spaces into $N_{s}$ and $N_{\Omega}$ bins, respectively, and by replacing the integrals and derivatives by their numerical approximations. The operator, $H$, then becomes an $N \times N$ matrix, where $N=N_{s} N_{\Omega}$.

Alternatively, the angular component of $\phi$ may be transformed into the basis of spherical harmonics, with only the $N_{\Omega}$ lowest angular frequencies kept as an approximation. In this case, the term $c \hat{p} \cdot \partial_{q} \phi(q, \hat{p})$ introduces a mixing between different spherical harmonics.

\section{C.4 The Diffusion Equation}

Let $\phi(q, \hat{p})$ be written as the sum of two terms:

$$
\phi(q, \hat{p})=\bar{\phi}(q)+\chi(q, \hat{p})
$$

where $\chi$ necessarily has the property that its average over all directions, $\hat{p}$, is zero. The equation for time evolution may be rewritten as:

$$
\dot{\bar{\phi}}+\dot{\chi}=-c \hat{p} \cdot \partial_{q} \bar{\phi}-c \hat{p} \cdot \partial_{q} \chi-\frac{1}{\tau} \chi
$$

This equation may be averaged over all directions, $\hat{p}$, to arrive at the rate of change of $\bar{\phi}$ alone:

$$
\dot{\bar{\phi}}=\left\langle-c \hat{p} \cdot \partial_{q} \chi\right\rangle
$$


where the brackets, $\langle$.$\rangle , represent an average over all \hat{p}$ directions. Equation C.20 may be substituted into equation C.19 to arrive at the rate of change of $\chi$ :

$$
\dot{\chi}=-c \hat{p} \cdot \partial_{q} \bar{\phi}-c \hat{p} \cdot \partial_{q} \chi+c\left\langle\hat{p} \cdot \partial_{q} \chi\right\rangle-\frac{1}{\tau} \chi
$$

Define the scattering length, $\lambda$, to be:

$$
\lambda \equiv c \tau
$$

In terms of $\lambda$ and $\tau$, we arrive at the equation:

$$
\left(\chi+\tau \dot{\chi}+\lambda \hat{p} \cdot \partial_{q} \chi-\lambda\left\langle\hat{p} \cdot \partial_{q} \chi\right\rangle\right)=-\lambda \hat{p} \cdot \partial_{q} \bar{\phi}
$$

If the temporal variation in $\chi$ is much longer than the scattering time, $\tau$, and if the spatial variation in $\chi$ is much longer than the scattering length, $\lambda$, then equation C.23 may be approximated as:

$$
\chi \approx-\lambda \hat{p} \cdot \partial_{q} \bar{\phi}
$$

The result of equation C.24 may be substituted back into equation C.20 to arrive at an approximate time evolution equation for $\bar{\phi}$ :

$$
\dot{\bar{\phi}} \approx c \lambda\left\langle\hat{p} \cdot \partial_{q} \hat{p} \cdot \partial_{q}\right\rangle \bar{\phi}=c \lambda \partial_{q} \cdot\langle P\rangle \cdot \partial_{q} \bar{\phi}
$$

where $P$ is the projection operator onto the direction $\hat{p}$. It is simple to show that the average of the projection operator over all directions of $\hat{p}$ is:

$$
\langle P\rangle=\frac{1}{D}
$$

where $D$ is the number of dimensions. Therefore, the approximate time evolution of $\bar{\phi}$ is:

$$
\dot{\bar{\phi}} \approx \frac{c \lambda}{D} \partial_{q}^{2} \bar{\phi}
$$

Here we see that the diffusion equation is recovered in the limit where the spatial and temporal variations in $\bar{\phi}$ are much larger than $\lambda$ and $\tau$, respectively. 


\section{Appendix D}

\section{Diffusion in a Lossy Medium}

In this section, the process of diffusion and absorption of particles is investigated in the case where the particles have a finite lifetime, $\tau$. The total number of particles absorbed by a set of perfectly absorbing bodies in $N$ dimensions due to a point source is calculated. The case of one or two imperfectly absorbing bodies is treated in one dimension, and a formula is derived for the fraction of particles absorbed by each absorber as a function of the position of the source. The case of imperfectly absorbing bodies is treated in two dimensions for the cylindrically symmetric case.

\section{D.1 The Diffusion Equation}

Consider an infinite isotropic and homogeneous medium in which particles diffuse with diffusion coefficient, $D$. Let it furthermore be assumed that the particles have a finite lifetime, $\tau$. The number density of particles, $n$, will obey the differential equation:

$$
\dot{n}=D \nabla^{2} n-\frac{n}{\tau}+s
$$

where $s$ represents a source of particles. The average current density of particles, $J$, is proportional to the gradient in the number density:

$$
J=-D \nabla n
$$


The total flux of particles, $\Phi$, leaving a closed surface, $\mathcal{S}$, is given by the surface integral of $J$ over the surface:

$$
\Phi=\oint_{\mathcal{S}} d \mathcal{A} \cdot J
$$

Assume that the medium contains absorbing bodies of arbitrary shape. Each absorber obeys the boundary condition:

$$
d \mathcal{A} \cdot J=-D d \mathcal{A} \cdot \nabla n=-|d \mathcal{A}| n v
$$

where $v$ is the absorption coefficient, which has units of velocity. In terms of $D$ and $v$, a characteristic absorption length, $l_{\mathrm{a}}$, may be defined for each absorber:

$$
l_{\mathrm{a}} \equiv \frac{D}{v}
$$

\section{D.2 The Integrated Absorption}

Assume that the source of particles, $s$, is only non-zero for a finite time and only over a finite volume of space. This source will create a total quantity of particles, $q$, which will diffuse through the medium. Some quantity, $Q_{i}$, will be absorbed on the $i$ th absorber. This quantity is the integral over all time of the flux through the surface of that absorber:

$$
Q_{i}=\int_{-\infty}^{\infty} d t \Phi_{i}=\int_{-\infty}^{\infty} d t \oint_{\mathcal{S}_{i}} d \mathcal{A} \cdot J
$$

Note that this quantity will be negative, as it represents absorption rather than emission of particles. Let the scalar field, $\phi$, be defined to be:

$$
\phi(x) \equiv \int_{-\infty}^{\infty} d t n(x, t)
$$

and let the vector field, $E$, be defined to be:

$$
E \equiv-\nabla \phi
$$


In terms of these, the quantity of particles absorbed by the $i$ th absorber is:

$$
\frac{Q_{i}}{D}=\oint_{\mathcal{S}_{i}} d \mathcal{A} \cdot E
$$

Therefore, $Q_{i}$, depends only on the scalar field $\phi$, and not on the detailed timedependance of $n$. Using the method described in reference [76], an equation for $\phi$ may be found by integrating equation D.1 with respect to time:

$$
\begin{aligned}
\int_{-\infty}^{\infty} d t \dot{n}=0 & =D \nabla^{2} \int_{-\infty}^{\infty} d t n-\frac{1}{\tau} \int_{-\infty}^{\infty} d t n+\int_{-\infty}^{\infty} d t s \\
0 & =D \nabla^{2} \phi-\frac{\phi}{\tau}+\rho
\end{aligned}
$$

where the left-hand side vanishes because $n(x,-\infty)=n(x, \infty)=0$, and the source density, $\rho$, is defined to be:

$$
\rho(x) \equiv \int_{-\infty}^{\infty} d t s(x, t)
$$

The equation relating $\phi$ and $\rho$ is:

$$
\left(\nabla^{2}-\frac{1}{l_{\mathrm{d}}^{2}}\right) \phi=-\frac{\rho}{D}
$$

where the characteristic diffusion length, $l_{\mathrm{d}}$, is defined to be:

$$
l_{\mathrm{d}} \equiv \sqrt{D \tau}
$$

Equation I.37 may also be written as:

$$
\hat{\mathcal{O}} \phi=-\frac{\rho}{D}
$$

where the operator, $\hat{\mathcal{O}}$, is:

$$
\hat{\mathcal{O}} \equiv\left(\nabla^{2}-\frac{1}{l_{\mathrm{d}}^{2}}\right)
$$


APPENDIX D. DIFFUSION IN A LOSSY MEDIUM

\section{D.3 A Point Source in the Presence of Perfect Ab- sorbers}

Consider the case where all absorbing bodies are perfect absorbers. In this case, $v=\infty$ and $l_{\mathrm{a}}=0$. The boundary condition at an absorbing surface is:

$$
n=-l_{\mathrm{a}} \frac{d \mathcal{A}}{|d \mathcal{A}|} \cdot \nabla n=0
$$

which implies that $\phi=0$ on the boundaries of all absorbing bodies.

Let the particle source have the form:

$$
s(x, t)=q \delta^{N}(x-r) \delta(t)
$$

where $N$ is the number of dimensions. The density due to this source is:

$$
\rho(x)=q \delta^{N}(x-r)
$$

To find the total absorption on the $i$ th absorber due to this source, one must solve equation I.37 for $\phi$, and then perform the integral in equation D.9.

This calculation may be simplified by means of a technique similar to that used in the proof of the Shockley-Ramo theorem. Consider two configurations, $\hat{\mathcal{O}} \phi^{(1)}=$ $-\rho^{(1)} / D$, and $\hat{\mathcal{O}} \phi^{(2)}=-\rho^{(2)} / D$. Due to the symmetry of the operator $\hat{\mathcal{O}}$, a reciprocity relation holds between the two configurations:

$$
\begin{aligned}
\int d \mathcal{V} \phi^{(1)} \rho^{(2)} & =-D \int d \mathcal{V} \phi^{(1)}\left(\nabla^{2}-\frac{1}{l_{\mathrm{d}}^{2}}\right) \phi^{(2)} \\
& =-D \int d \mathcal{V} \phi^{(2)}\left(\nabla^{2}-\frac{1}{l_{\mathrm{d}}^{2}}\right) \phi^{(1)} \\
& =\int d \mathcal{V} \phi^{(2)} \rho^{(1)}
\end{aligned}
$$

which can be proved by means of integration by parts.

Consider two configurations like those shown in Figure D.1. Configuration (1) is that which we wish to solve, i.e. a point source of magnitude $q$ in the presence of 
(1)

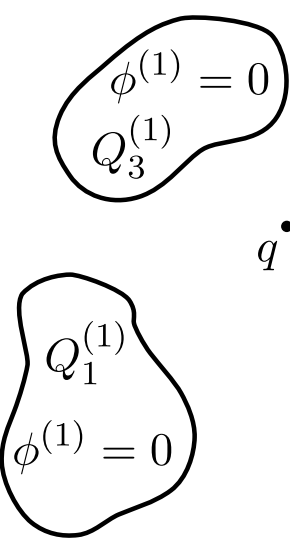

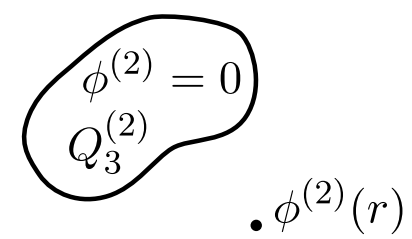

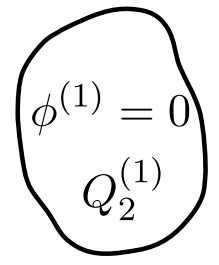

$(2)$

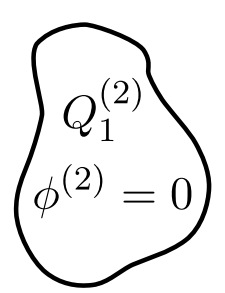

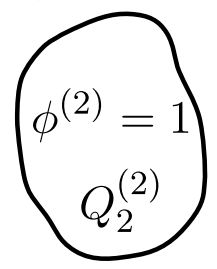

Figure D.1: Two configurations of $\phi$ and $\rho$. On the left, a point source of particles, $q$, is located at position, $r$. The field $\phi^{(1)}$ is zero on the surfaces of all absorbers. On the right, there is no source at position, $r$, but the field, $\phi^{(2)}$, at location $r$ is non-zero due to the modified boundary condition of absorber 2 .

perfect absorbers having the boundary condition $\phi=0$. In the second configuration, there is no point source, and the $i$ th absorber is given a boundary condition of $\phi=1$, while the rest have the boundary condition $\phi=0$.

In configuration (2), the volume surrounding the absorbers will be filled with the field $\phi^{(2)}$, generated by the $i$ th absorber, which satisfies the equation $\hat{\mathcal{O}} \phi=0$ in the volume between absorbers. In general, let $\phi_{i}$ denote the field generated where $\rho=0$ and where $\phi=0$ on the boundaries of the absorbers, except for the $i$ th absorber, for which the boundary condition is $\phi=1$.

Using equation I.38, we may calculate the quantity, $Q_{i}$, of particles absorbed onto the $i$ th absorber. The integrals of equation I.38 are:

$$
\int d \mathcal{V} \phi^{(1)} \rho^{(2)}=0=\int d \mathcal{V} \phi^{(2)} \rho^{(1)}=q \phi^{(2)}(r)+Q_{i}
$$

Therefore, the quantity of particles absorbed by the $i$ th absorber may be determined by the product of the source magnitude, $q$, and the $i$ th absorber field:

$$
Q_{i}=-q \phi_{i}(r)
$$




\section{D.4 A Point Source in One Dimension}

Consider a finite region of space in one dimension of width $w$, with particle absorbers located at $-w / 2$ and $+w / 2$. Let the particle source have the form:

$$
s=q \delta(x-r) \delta(t)
$$

The corresponding density, $\rho$, is:

$$
\rho(x)=q \delta(x-r)
$$

The field, $\phi$, will obey the equation:

$$
\left(\partial_{x}^{2}-\frac{1}{l_{\mathrm{d}}^{2}}\right) \phi=0
$$

in the regions $-w / 2<x<r$, and $r<x<w / 2$. A general solution to this equation has the form:

$$
\phi=C_{1} e^{x / l_{\mathrm{d}}}+C_{2} e^{-x / l_{\mathrm{d}}}
$$

Define two fields, $\phi_{L}$ and $\phi_{R}$, each of which solves equation D.24 in the left and right regions respectively:

$$
\begin{aligned}
& \phi_{L}(x)=L_{1} e^{x / l_{\mathrm{d}}}+L_{2} e^{-x / l_{\mathrm{d}}} \\
& \phi_{R}(x)=R_{1} e^{x / l_{\mathrm{d}}}+R_{2} e^{-x / l_{\mathrm{d}}}
\end{aligned}
$$

The full field, $\phi$, is:

$$
\phi(x)=\Theta(x-r) \phi_{R}(x)+\Theta(r-x) \phi_{L}(x)
$$

where $\Theta$ is the Heaviside step function. The first derivative of $\phi$ is:

$$
\begin{aligned}
\phi^{\prime}(x)= & \delta(x-r) \phi_{R}(r)+\Theta(x-r) \phi_{R}^{\prime}(x) \\
& -\delta(x-r) \phi_{L}(r)+\Theta(r-x) \phi_{L}^{\prime}(x)
\end{aligned}
$$


The second derivative of $\phi$ is:

$$
\begin{aligned}
\phi^{\prime \prime}(x)= & \delta^{\prime}(x-r) \phi_{R}(r)+\delta(x-r) \phi_{R}^{\prime}(r)+\Theta(x-r) \phi_{R}^{\prime \prime}(x) \\
& -\delta^{\prime}(x-r) \phi_{L}(r)-\delta(x-r) \phi_{L}^{\prime}(r)+\Theta(r-x) \phi_{L}^{\prime \prime}(x)
\end{aligned}
$$

Rearranging equation D.30 results in:

$$
\begin{aligned}
\phi^{\prime \prime}(x)-\frac{1}{l_{\mathrm{d}}^{2}} \phi(x)= & \delta^{\prime}(x-r)\left(\phi_{R}(r)-\phi_{L}(r)\right) \\
& +\delta(x-r)\left(\phi_{R}^{\prime}(r)-\phi_{L}^{\prime}(r)\right) \\
= & -\frac{q}{D} \delta(x-r)
\end{aligned}
$$

Therefore, at $x=r$, we have the conditions:

$$
\begin{aligned}
\phi_{R}(r)-\phi_{L}(r) & =0 \\
\phi_{R}^{\prime}(r)-\phi_{L}^{\prime}(r) & =-\frac{q}{D}
\end{aligned}
$$

We must also impose the following boundary conditions at the locations of the absorbers:

$$
\begin{aligned}
\phi_{L}(-w / 2) & =l_{\mathrm{al}} \phi_{L}^{\prime}(-w / 2) \\
\phi_{R}(w / 2) & =-l_{\mathrm{ar}} \phi_{R}^{\prime}(w / 2)
\end{aligned}
$$

where $l_{\mathrm{al}}$ and $l_{\mathrm{ar}}$ are the left and right absorption lengths respectively. The conditions in equations D.32 and D.33 provide four constraints from which the the four unknowns, $L_{1}, L_{2}, R_{1}$, and $R_{2}$, may be determined.

Based on this solution, the quantities of particles absorbed by the left and right absorbers, $Q_{L}$ and $Q_{R}$, are:

$$
\begin{aligned}
Q_{L} & =-D \phi^{\prime}(-w / 2)=-q F_{L} \\
Q_{R} & =D \phi^{\prime}(w / 2)=-q F_{R}
\end{aligned}
$$

where $F_{L}(\xi)$ and $F_{R}(\xi)$ represent the fractional absorption into the left and right 
absorbers, respectively, as functions of position:

$$
\begin{aligned}
& F_{L}(\xi)= \frac{\lambda_{\mathrm{d}}\left(\lambda_{\mathrm{ar}} \cosh \left(\frac{1-2 \xi}{2 \lambda_{\mathrm{d}}}\right)+\lambda_{\mathrm{d}} \sinh \left(\frac{1-2 \xi}{2 \lambda_{\mathrm{d}}}\right)\right)}{\lambda_{\mathrm{d}}\left(\lambda_{\mathrm{al}}+\lambda_{\mathrm{ar}}\right) \cosh \left(\frac{1}{\lambda_{\mathrm{d}}}\right)+\left(\lambda_{\mathrm{d}}^{2}+\lambda_{\mathrm{al}} \lambda_{\mathrm{ar}}\right) \sinh \left(\frac{1}{\lambda_{\mathrm{d}}}\right)} \\
& F_{R}(\xi)=\frac{\lambda_{\mathrm{d}}\left(\lambda_{\mathrm{al}} \cosh \left(\frac{1+2 \xi}{2 \lambda_{\mathrm{d}}}\right)+\lambda_{\mathrm{d}} \sinh \left(\frac{1+2 \xi}{2 \lambda_{\mathrm{d}}}\right)\right)}{\lambda_{\mathrm{d}}\left(\lambda_{\mathrm{al}}+\lambda_{\mathrm{ar}}\right) \cosh \left(\frac{1}{\lambda_{\mathrm{d}}}\right)+\left(\lambda_{\mathrm{d}}^{2}+\lambda_{\mathrm{al}} \lambda_{\mathrm{ar}}\right) \sinh \left(\frac{1}{\lambda_{\mathrm{d}}}\right)}
\end{aligned}
$$

and the dimensionless variables, $\xi, \lambda_{\mathrm{d}}, \lambda_{\mathrm{al}}$, and $\lambda_{\mathrm{ar}}$, are defined to be:

$$
\begin{aligned}
\xi & \equiv r / w \\
\lambda_{\mathrm{d}} & \equiv l_{\mathrm{d}} / w \\
\lambda_{\mathrm{al}} & \equiv l_{\mathrm{al}} / w \\
\lambda_{\mathrm{ar}} & \equiv l_{\mathrm{ar}} / w
\end{aligned}
$$

\section{D.5 Mean Collection Fraction in One Dimension}

Consider the case in which energy is deposited in the diffusive medium with a uniform distribution. The mean fractional energy collection may be found by averaging the collection fraction functions over all positions, $\xi$. The results are:

$$
\begin{aligned}
& F_{L}=\int_{-1 / 2}^{1 / 2} F_{L}(\xi) d \xi=\frac{\lambda_{\mathrm{d}}^{2}\left(\lambda_{\mathrm{ar}}+\lambda_{\mathrm{d}} \tanh \left(\frac{1}{2 \lambda_{\mathrm{d}}}\right)\right) \sinh \left(\frac{1}{\lambda_{\mathrm{d}}}\right)}{\lambda_{\mathrm{d}}\left(\lambda_{\mathrm{al}}+\lambda_{\mathrm{ar}}\right) \cosh \left(\frac{1}{\lambda_{\mathrm{d}}}\right)+\left(\lambda_{\mathrm{d}}^{2}+\lambda_{\mathrm{al}} \lambda_{\mathrm{ar}}\right) \sinh \left(\frac{1}{\lambda_{\mathrm{d}}}\right)} \\
& F_{R}=\int_{-1 / 2}^{1 / 2} F_{R}(\xi) d \xi=\frac{\lambda_{\mathrm{d}}^{2}\left(\lambda_{\mathrm{al}}+\lambda_{\mathrm{d}} \tanh \left(\frac{1}{2 \lambda_{\mathrm{d}}}\right)\right) \sinh \left(\frac{1}{\lambda_{\mathrm{d}}}\right)}{\lambda_{\mathrm{d}}\left(\lambda_{\mathrm{al}}+\lambda_{\mathrm{ar}}\right) \cosh \left(\frac{1}{\lambda_{\mathrm{d}}}\right)+\left(\lambda_{\mathrm{d}}^{2}+\lambda_{\mathrm{al}} \lambda_{\mathrm{ar}}\right) \sinh \left(\frac{1}{\lambda_{\mathrm{d}}}\right)}
\end{aligned}
$$

In the special case where one boundary is perfectly reflecting, the absorption length for that side is infinite. Therefore, the mean one-sided absorption fraction, $F$, for a diffusive medium with an absorption length of $\lambda_{\mathrm{a}}$ may be found by taking the 
limit of $F_{L}$ as $\lambda_{\text {ar }}$ approaches infinity, evaluated at the point $\lambda_{\mathrm{al}}=\lambda_{\mathrm{a}}$ :

$$
F=\left.\lim _{\lambda_{\mathrm{ar}} \rightarrow \infty} F_{L}\right|_{\lambda_{\mathrm{al}}=\lambda_{\mathrm{a}}}=\frac{\lambda_{\mathrm{d}}^{2} \sinh \left(\frac{1}{\lambda_{\mathrm{d}}}\right)}{\lambda_{\mathrm{a}} \sinh \left(\frac{1}{\lambda_{\mathrm{d}}}\right)+\lambda_{\mathrm{d}} \cosh \left(\frac{1}{\lambda_{\mathrm{d}}}\right)}=\frac{\lambda_{\mathrm{d}}^{2}}{\lambda_{\mathrm{a}}+\lambda_{\mathrm{d}} \operatorname{coth}\left(\frac{1}{\lambda_{\mathrm{d}}}\right)}
$$

\section{D.6 Mean Collection Fraction in an Annulus}

Consider a diffusive medium shaped like an annulus, with inner radius, $r_{1}$, and outer radius, $r_{2}$, and with an absorbing boundary on the inner circumference and a reflecting boundary on the outer circumference, as shown in Figure D.2.

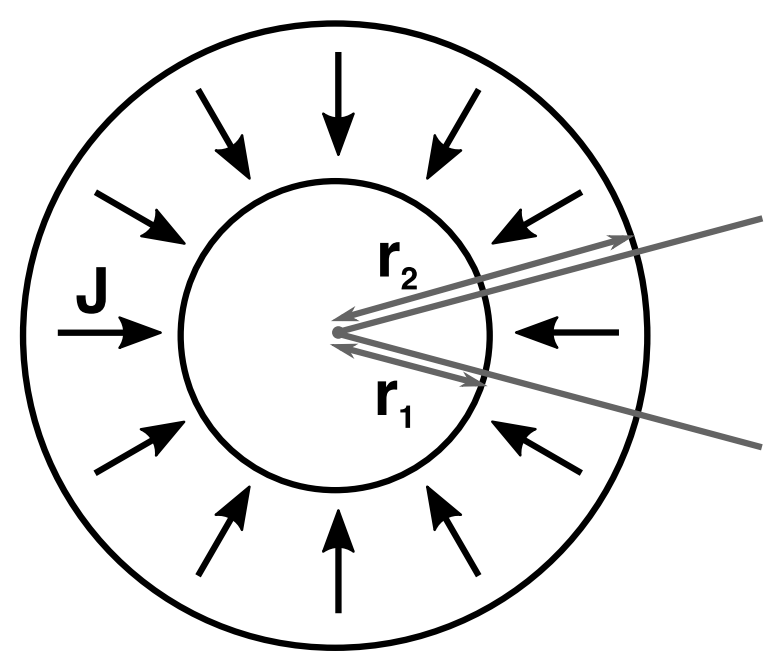

Figure D.2: A diffusive medium shaped like an annulus, with inner radius, $r_{1}$, and outer radius, $r_{2}$, and with a homogeneous source of particles distributed throughout the medium, creating a steady-state two-dimensional current density of particles, $J$.

Assume that a homogeneous particle source fills the entire diffusive medium. In this case, the geometry and particle source both have cylindrical symmetry. Consequently, the field, $\phi$ will also have cylindrical symmetry, and will satisfy the equation:

$$
\nabla^{2} \phi-\frac{\phi}{l_{\mathrm{d}}^{2}}=-\frac{\sigma}{D}
$$


where the two-dimensional source density, $\sigma$, is:

$$
\sigma=\frac{Q}{\pi\left(r_{2}^{2}-r_{1}^{2}\right)}
$$

where $Q$ is the total number of particles. In cylindrical coordinates, the 2D Laplace operator is:

$$
\nabla^{2} f=\frac{1}{r} \frac{\partial}{\partial r}\left(r \frac{\partial f}{\partial r}\right)+\frac{1}{r^{2}} \frac{\partial^{2} f}{\partial \theta^{2}}
$$

Due to the cylindrical symmetry, we may assume that $\phi$ is constant with respect to the azimuthal angle, $\theta$. Therefore, equation D.39 may be written as:

$$
\frac{\partial^{2} \phi}{\partial r^{2}}+\frac{1}{r} \frac{\partial \phi}{\partial r}-\frac{\phi}{l_{\mathrm{d}}^{2}}=-\frac{\sigma}{D}
$$

which can be rearranged in the following way:

$$
r^{2} \frac{\partial^{2} \phi}{\partial r^{2}}+r \frac{\partial \phi}{\partial r}-r^{2} \frac{\phi-\sigma l_{\mathrm{d}}^{2} / D}{l_{\mathrm{d}}^{2}}=0
$$

A general solution to this equation has the form:

$$
\phi(r)=\frac{\sigma l_{\mathrm{d}}^{2}}{D}+C_{1} I_{0}\left(\frac{r}{l_{\mathrm{d}}}\right)+C_{2} K_{0}\left(\frac{r}{l_{\mathrm{d}}}\right)
$$

where $C_{1}$ and $C_{2}$ are undetermined constants, and $I_{0}$ and $K_{0}$ are modified Bessel functions of the first and second kind, respectively. The constants, $C_{1}$ and $C_{2}$, are fixed by the two boundary conditions:

$$
\begin{aligned}
\phi^{\prime}\left(r_{2}\right) & =0 \\
l_{\mathrm{a}} \phi^{\prime}\left(r_{1}\right) & =\phi\left(r_{1}\right)
\end{aligned}
$$


where $l_{\mathrm{a}}$ is the absorption length of the absorbing boundary on the inner circumference. Solving for $C_{1}$ and $C_{2}$, we get:

$$
\begin{aligned}
C_{1} & =-\frac{l_{\mathrm{d}}^{2} \sigma}{D} \frac{K_{1}\left(\rho_{2}\right)}{I_{1}\left(\rho_{2}\right)\left(K_{0}\left(\rho_{1}\right)+\lambda_{\mathrm{a}} K_{1}\left(\rho_{1}\right)\right)+K_{1}\left(\rho_{2}\right)\left(I_{0}\left(\rho_{1}\right)-\lambda_{\mathrm{a}} I_{1}\left(\rho_{1}\right)\right)} \\
C_{2} & =-\frac{l_{\mathrm{d}}^{2} \sigma}{D} \frac{I_{1}\left(\rho_{2}\right)}{I_{1}\left(\rho_{2}\right)\left(K_{0}\left(\rho_{1}\right)+\lambda_{\mathrm{a}} K_{1}\left(\rho_{1}\right)\right)+K_{1}\left(\rho_{2}\right)\left(I_{0}\left(\rho_{1}\right)-\lambda_{\mathrm{a}} I_{1}\left(\rho_{1}\right)\right)}
\end{aligned}
$$

where the dimensionless quantities, $\rho_{1}, \rho_{2}$, and $\lambda_{\mathrm{a}}$, are defined to be:

$$
\begin{aligned}
\rho_{1} & \equiv r_{1} / l_{\mathrm{d}} \\
\rho_{2} & \equiv r_{2} / l_{\mathrm{d}} \\
\lambda_{\mathrm{a}} & \equiv l_{\mathrm{a}} / l_{\mathrm{d}}
\end{aligned}
$$

The quantity of particles absorbed on the inner boundary is:

$$
\begin{aligned}
Q_{\mathrm{a}} & =2 \pi r_{1} D \phi^{\prime}\left(r_{1}\right) \\
& =2 \pi \rho_{1} l_{\mathrm{d}}^{2} \sigma \frac{I_{1}\left(\rho_{2}\right) K_{1}\left(\rho_{1}\right)-I_{1}\left(\rho_{1}\right) K_{1}\left(\rho_{2}\right)}{I_{1}\left(\rho_{2}\right)\left(K_{0}\left(\rho_{1}\right)+\lambda_{\mathrm{a}} K_{1}\left(\rho_{1}\right)\right)+K_{1}\left(\rho_{2}\right)\left(I_{0}\left(\rho_{1}\right)-\lambda_{\mathrm{a}} I_{1}\left(\rho_{1}\right)\right)}
\end{aligned}
$$

The fraction of particles absorbed is:

$$
F=\frac{Q_{\mathrm{a}}}{Q}=\frac{2 \rho_{1}}{\rho_{2}^{2}-\rho_{1}^{2}} \frac{I_{1}\left(\rho_{2}\right) K_{1}\left(\rho_{1}\right)-I_{1}\left(\rho_{1}\right) K_{1}\left(\rho_{2}\right)}{I_{1}\left(\rho_{2}\right)\left(K_{0}\left(\rho_{1}\right)+\lambda_{\mathrm{a}} K_{1}\left(\rho_{1}\right)\right)+K_{1}\left(\rho_{2}\right)\left(I_{0}\left(\rho_{1}\right)-\lambda_{\mathrm{a}} I_{1}\left(\rho_{1}\right)\right)}
$$

Finally, note that the diffusion current, $J$, has only a radial component. Therefore, we may take a wedge-shaped slice out of the annulus, as shown in Figure D.2, and the new edges formed by the cut will obey the boundary condition of a perfectly reflecting boundary. Therefore, the collection fraction for a wedge-shaped slice of an annulus is also given by equation D.49. 


\section{D.7 Comparison of $1 \mathrm{D}$ and 2D Solutions}

In order to compare the collection fraction in equation D.49 to that in equation D.38, we may use the following asymptotic forms of the Bessel functions:

$$
\begin{aligned}
I_{\alpha}(z) & \approx \frac{e^{z}}{\sqrt{2 \pi z}} \\
K_{\alpha}(z) & \approx \sqrt{\frac{\pi}{2 z}} e^{-z}
\end{aligned}
$$

Applying these substitutions to equation D.49, we get:

$$
\begin{aligned}
F & \approx \frac{2 \rho_{1}}{\rho_{2}^{2}-\rho_{1}^{2}} \frac{\sinh \left(\rho_{2}-\rho_{1}\right)}{\lambda_{a} \sinh \left(\rho_{2}-\rho_{1}\right)+\lambda_{d} \cosh \left(\rho_{2}-\rho_{1}\right)} \\
& \approx \frac{2 l_{\mathrm{d}}^{2} r_{1}}{r_{2}^{2}-r_{1}^{2}} \frac{1}{l_{\mathrm{a}}+l_{\mathrm{d}} \operatorname{coth}\left(\frac{r_{2}-r_{1}}{l_{\mathrm{d}}}\right)}
\end{aligned}
$$

for $r_{2}>r_{1} \gg l_{\mathrm{d}}$. In the limit where $r_{2}=r_{1}+w$ and $r_{1} \rightarrow \infty$, the wedge approaches a rectangular shape with width $w$. In this limit, $F$ becomes:

$$
F \approx \frac{\left(\frac{l_{\mathrm{d}}}{w}\right)^{2}}{\left(\frac{l_{\mathrm{a}}}{w}\right)+\left(\frac{l_{\mathrm{d}}}{w}\right) \operatorname{coth}\left(\frac{w}{l_{\mathrm{d}}}\right)}
$$

which is equivalent to equation D.38, as would be expected. 


\section{Appendix E}

\section{Linear TES Response}

In this section, the linear response of a transition-edge sensor to a small perturbation is found. From this result, a relation is derived between the total energy deposited in the transition-edge sensor and the integrated deviation in the electrical power from its steady-state value.

\section{E.1 The Electro-Thermal Feedback of a Transition- Edge Sensor}

A transition-edge sensor consists of a thin film of conducting material which transitions to a superconducting phase below a critical temperature, $T_{c}$. The conducting film rests upon on a non-conducting substrate at a temperature $T_{s}$. Because the electron system in the conductive film is only weakly coupled to the phonon system within the insulating substrate, it may be assumed that at any point in time the electron system in the film is in thermal equilibrium at a uniform temperature, $T$, even though $T$ may differ from $T_{s}$.

If $T$ is greater than $T_{s}$, the conducting film will loose energy to the substrate. According to reference [77], the rate of energy loss is:

$$
P_{\text {loss }}=K\left(T^{n}-T_{s}^{n}\right)
$$


In a typical implementation of a transition-edge sensor, a voltage source with voltage, $V$, and output resistance, $R_{o}$, is connected across the conducting film. The electrical power dissipated in the film is:

$$
P_{\text {diss }}=\left(\frac{V}{R+R_{o}}\right)^{2} R
$$

where $R$ is the resistance of the film. The derivative of the dissipated power with respect to resistance is:

$$
\frac{d P_{\text {diss }}}{d R}=\frac{V^{2}}{\left(R+R_{o}\right)^{2}}-2 \frac{V^{2}}{\left(R+R_{o}\right)^{3}} R=\frac{P_{\text {diss }}}{R}\left(1-2 \frac{R}{R+R_{o}}\right)=-\rho \frac{P_{\text {diss }}}{R}
$$

where the dimensionless quantity, $\rho$, is defined to be:

$$
\rho \equiv \frac{R-R_{o}}{R+R_{o}}=\frac{1-r}{1+r}
$$

and where the dimensionless quantity, $r$, is defined to be:

$$
r \equiv \frac{R_{o}}{R}
$$

Near the critical temperature the resistance varies rapidly with small changes in temperature. If the temperature of the substrate is lower than the critical temperature, and if $R>R_{o}$, electro-thermal feedback will maintain the film at a constant temperature close to $T_{c}$, as any increase in $T$ will cause an increase in $R$, and a decrease in $P_{\text {diss }}$, while a decrease in $T$ will cause the opposite effect.

\section{E.2 The Resistance vs. Temperature}

In general, the resistance of the conducting film will be a function of both its temperature, and the current, $I$, passing through it:

$$
R=R(T, I)
$$


Define the dimensionless quantities, $\alpha$ and $\beta$, to be the following partial derivatives:

$$
\begin{aligned}
\alpha & \equiv \frac{\partial \ln R}{\partial \ln T} \\
\beta & \equiv \frac{\partial \ln R}{\partial \ln I}
\end{aligned}
$$

In the biased state, the following relation holds between the current and the resistance:

$$
\begin{aligned}
I & =\frac{V}{R+R_{o}} \\
d I & =-\frac{V}{\left(R+R_{o}\right)^{2}} d R \\
d \ln I & =-\sigma d \ln R
\end{aligned}
$$

where the dimensionless quantity, $\sigma$, is defined to be:

$$
\sigma \equiv \frac{R}{R+R_{o}}=\frac{1}{1+r}
$$

This constraint leads to the following relation between the total differentials of $\ln R$ and $\ln T$ :

$$
\begin{aligned}
d \ln R & =\alpha d \ln T+\beta d \ln I \\
& =\alpha d \ln T-\beta \sigma d \ln R \\
(1+\beta \sigma) d \ln R & =\alpha d \ln T
\end{aligned}
$$

Let the dimensionless quantity, $\gamma$, be defined to be following total derivative:

$$
\gamma \equiv \frac{d \ln R}{d \ln T}
$$

Gamma may be expressed in terms of $\alpha$ and $\beta$ as follows:

$$
\gamma=\frac{d \ln R}{d \ln T}=\frac{\alpha}{1+\beta \sigma}=\frac{\alpha(1+r)}{1+r+\beta}
$$




\section{E.3 The Dynamics of a Transition-Edge Sensor}

The film will reach a steady-state at a temperature where $P_{\text {diss }}=P_{\text {loss }}$. Define $T_{0}$ to be the temperature at which this equality holds, and define $P_{0}$ to be equal the steady-state power loss or dissipation:

$$
P_{0} \equiv P_{\text {loss }}=P_{\text {diss }}
$$

The response of the film to the addition of a small quantity of energy may be approximated by taking the first-order Taylor expansion of the power loss and dissipation functions at the temperature $T_{0}$. Let the symbol $\delta$ denote the change in a quantity from its steady-state value. The first-order change in $P_{\text {loss }}$ is:

$$
\delta P_{\text {loss }}=n K T_{0}^{n-1} \delta T=G \delta T
$$

where $G$, defined by:

$$
G \equiv n K T_{0}^{n-1}
$$

is the coefficient of thermal conduction. The first-order change in $P_{\text {diss }}$ is:

$$
\delta P_{\text {diss }}=-\rho P_{0} \frac{\delta R}{R}=-\gamma \rho P_{0} \frac{\delta T}{T_{0}}=-\zeta P_{0} \frac{\delta T}{T_{0}}
$$

where the dimensionless quantity, $\zeta$, is defined to be:

$$
\zeta \equiv \gamma \rho=\frac{\alpha(1-r)}{1+\beta+r}
$$

As long as the electron system remains in a state of quasi-equilibrium, any change in its internal energy, $U$, will be accompanied by a change in its temperature:

$$
\delta U=C \delta T
$$

where $C$ is the heat capacity. The internal energy will change with time according to 
the equation:

$$
\delta \dot{U}=\delta P_{\text {diss }}-\delta P_{\text {loss }}+P_{\text {ext }}
$$

where the dot denotes a time derivative, and $P_{\text {ext }}$ represents an external source of thermal power, separate from the electrical dissipation. Equation E.19 may be used to derive a first-order linear differential equation for $\delta T$ :

$$
\begin{aligned}
C \delta \dot{T} & =-\left(\zeta \frac{P_{0}}{T_{0}}+G\right) \delta T+P_{\mathrm{ext}} \\
\delta \dot{T} & =-\frac{G}{C}(L+1) \delta T+\frac{P_{\mathrm{ext}}}{C}
\end{aligned}
$$

where, following [1], the dimensionless quantity, $L$, is defined to be:

$$
L \equiv \frac{\zeta P_{0}}{T_{0} G}=\frac{\zeta K\left(T_{0}^{n}-T_{s}^{n}\right)}{n K T_{0}^{n}}=\frac{\zeta}{n}\left(1-\frac{T_{s}^{n}}{T_{0}^{n}}\right) \approx \frac{\zeta}{n}\left(1-\frac{T_{s}^{n}}{T_{c}^{n}}\right)
$$

The response of the temperature to an instantaneous deposition of energy, $P_{\text {ext }}=$ $\mathscr{E} \delta(t)$, is a decaying exponential:

$$
\delta T=\frac{\mathscr{E}}{C} e^{-t / \tau} \Theta(t)
$$

where $\Theta(t)$ is the Heaviside step-function, and the time constant, $\tau$, is given by:

$$
\tau=\frac{C}{G} \frac{1}{L+1}
$$

\section{E.4 The Loss of Deposited Energy to the Sub- strate}

For the duration of time that the conducting film is recovering from a small perturbation, its temperature deviates above the steady-state value. This necessarily implies that a certain quantity of energy, $\mathscr{E}_{s}$, is lost to the substrate over and above that amount which would have been lost had the film remained in its steady state. This 
quantity of energy may be calculated by integrating the deviation in the power loss, $\delta P_{\text {loss }}$, with respect to time:

$$
\begin{aligned}
\mathscr{E}_{s} & =\int_{-\infty}^{\infty} d t \delta P_{\text {loss }}=\int_{-\infty}^{\infty} d t G \delta T \\
& =\int_{-\infty}^{\infty} d t G \frac{\mathscr{E}}{C} e^{-t / \tau} \Theta(t) \\
& =\frac{G}{C} \tau \mathscr{E} \\
& =\frac{1}{L+1} \mathscr{E}
\end{aligned}
$$

\section{E.5 The Integrated Electrical Power}

While the conducting film is recovering from a small perturbation, the electric power dissipated by it is less than its steady-state value. The integral of this deficiency in power is easily measurable by electrical means, and may be used to define a quantity which may be called the integrated energy, $\mathscr{E}_{\text {int }}$ :

$$
\mathscr{E}_{\mathrm{int}} \equiv-\int_{-\infty}^{\infty} d t \delta P_{\mathrm{diss}}
$$

This transfer of energy, along with the others, causes the internal energy of the film to change with time. However, once the conducting film returns again to a steadystate, its internal energy must return to the same value as before the perturbation. Consequently, the integral of the rate of change of the internal energy must be zero. Using this fact, and equation E.19, a relation may be derived between $\mathscr{E}_{\text {int }}$ and the total deposited energy, $\mathscr{E}$ :

$$
\begin{gathered}
\int_{-\infty}^{\infty} d t \delta \dot{U}=0=\int_{-\infty}^{\infty} d t\left(\delta P_{\text {diss }}-\delta P_{\text {loss }}+\delta P_{\text {ext }}\right) \\
\mathscr{E}_{\text {int }} \equiv-\int_{-\infty}^{\infty} d t \delta P_{\text {diss }}=\int_{-\infty}^{\infty} d t\left(-\delta P_{\text {loss }}+\delta P_{\text {ext }}\right)=-\mathscr{E}_{s}+\mathscr{E}
\end{gathered}
$$


Using the result of the previous section, we may conclude that the integrated deviation in electrical power is directly proportional to the deposited energy, with a constant of proportionality given by:

$$
\mathscr{E}_{\text {int }}=\frac{L}{L+1} \mathscr{E}
$$




\section{Appendix F}

\section{Electron-Phonon Interactions}

In this section, the differential rate of phonon emission and absorption by electrons in a semiconductor is derived in the continuum limit. The speed of sound and the electron mass are both allowed to be anisotropic. The differential emission and absorption rates are integrated to find the total emission and absorption rates in the case of an isotropic medium.

\section{F.1 Generalized Vectors}

In order to formulate Lagrangian mechanics in a way which may be applied simultaneously to both charge carriers and crystal vibrations in a uniform medium, it is useful to first summarize the common algebraic properties shared by the fields used to model these physical systems. Complex numbers, tensors, and functions of continuous variables can all be shown to satisfy the axioms of linear algebra over the field of real numbers. For this reason, these sets may each be treated as vector spaces. Each contains a generalized inner product, $a * b$, defined in the table below:

$\begin{array}{ll}\text { Complex Numbers: } & a * b \equiv \Re\left[a^{*} b\right] \\ \text { Geometric Vectors: } & a * b \equiv a \cdot b \\ \text { Tensors: } & \underline{a} * \underline{b} \equiv \operatorname{Tr}[\bar{a} b]=a^{i j} b_{j i} \\ \text { Functions: } & a * b \equiv \int d^{N} x a(x) b(x)\end{array}$

The inner product of two complex, vector, or tensor functions is defined to be the 
integral of the inner product of the two functions at each point.

Vectors may be written as the sum of scalar components, $a^{i}$, multiplied by basis elements, $\sigma_{i}$, using Einstein notation: $a=a^{i} \sigma_{i}$. (In the case of functions, this sum is an integral over a continuous index.) The metric is a matrix defined by: $g_{i j} \equiv \sigma_{i} * \sigma_{j}$. The matrix, $g^{i j}$, is defined to be the matrix inverse of $g_{i j}$, and the reciprocal basis is defined to be $\sigma^{i} \equiv g^{i j} \sigma_{j}$, such that, $\sigma^{i} * \sigma_{j}=\delta_{j}^{i}$, and the scalar components, $a_{i}=g_{i j} a^{j}$, are defined such that $a=a_{i} \sigma^{i}$. The derivative with respect to a vector, $a$, is defined to be:

$$
\partial_{a} \equiv \sigma^{i} \frac{\partial}{\partial_{a^{i}}}
$$

and has the property that:

$$
\partial_{a} a * b=b
$$

or in index notation:

$$
\partial_{a^{i}} a^{j} b_{j}=b_{i}
$$

For geometric vectors, the vector derivative is simply the gradient operator. For functions, the vector derivative is the functional derivative.

See reference [78] for a more detailed description of the properties of vector derivatives.

\section{F.2 Euler-Lagrange Equations}

The classical configuration of a system may be represented by a generalized vector, $q$. The Lagrangian, $\mathscr{L}(q, \dot{q})$, is a real, scalar function of the two vectors, $q$ and $\dot{q}$. The action, $\mathcal{S}$, is defined as:

$$
\mathcal{S}=\int d t \mathscr{L}(q, \dot{q})
$$


The first-order variation in the action, $\delta \mathcal{S}$, with respect to a variation in the path, $\delta q(t)$, is:

$$
\begin{aligned}
\delta \mathcal{S} & =\int d t\left(\delta q * \partial_{q} \mathscr{L}+\delta \dot{q} * \partial_{\dot{q}} \mathscr{L}\right) \\
& =\int d t \delta q *\left(\partial_{q} \mathscr{L}-d_{t} \partial_{\dot{q}} \mathscr{L}\right)
\end{aligned}
$$

where the usual trick of integration by parts has been employed. The conjugate momentum is defined by:

$$
p \equiv \partial_{\dot{q}} \mathscr{L}
$$

From the requirement that the action be extremized, or $\delta \mathcal{S}=0$ for all $\delta q$, we arrive at the Euler-Lagrange equation:

$$
\dot{p}=\partial_{q} \mathscr{L}
$$

The Hamiltonian, $\mathscr{H}$, may be derived from the Lagrangian by the Legandre transformation with respect to $\dot{q}$ :

$$
\mathscr{H}=p * \dot{q}-\mathscr{L}
$$

\section{F.3 Linear Operators}

Linear operators may be defined as linear functions which map vectors to vectors in the generalized vector space of functions. For example, take a linear operator, $\underline{O}$, to be the Laplacian operator, $\nabla^{2}$. We may represent the action of this operator on some scalar field, $a(x)$, in the following way:

$$
\underline{O} * a \equiv \nabla^{2} a(x)
$$

We may take the generalized inner product of the result with the scalar field $b(x)$ :

$$
b * \underline{O} * a=\int d^{N} x b(x) \nabla^{2} a(x)
$$


The transpose of the operator, $\bar{O}$, is a linear operator defined such that:

$$
b * \underline{O} * a=a * \bar{O} * b
$$

We may find this operator through integration by parts (assuming the boundary terms may be ignored):

$$
\begin{aligned}
b * \underline{O} * a & =\int d^{N} x b(x) \nabla^{2} a(x) \\
& =-\int d^{N} x(\nabla b(x)) \cdot(\nabla a(x)) \\
& =\int d^{N} x a(x) \nabla^{2} b(x) \\
& =a * \underline{O} * b
\end{aligned}
$$

Therefore, we conclude that $\underline{O}$ is a symmetric operator: $\bar{O}=\underline{O}$.

\section{F.4 The Schrödinger Lagrangian}

As described in reference [79], the Lagrangian for a complex scalar field whose EulerLagrange equation of motion is the Schrödinger equation is:

$$
\mathscr{L}=\int d^{3} x \Re\left[i \dot{\psi} \psi^{*}-\frac{1}{2 m}(\nabla \psi)^{*} \cdot(\nabla \psi)-U \psi^{*} \psi\right]
$$

where $U(x)$ is the potential, and where the units are chosen such that $\hbar=1$. Using linear operator notation, this Lagrangian may be written as:

$$
\mathscr{L}=\dot{\psi} * \underline{A} * \psi-\psi * \underline{B} * \psi
$$

where the operator, $\underline{A}$, is defined as:

$$
\underline{A} * \psi \equiv-i \psi
$$


the operator, $\underline{B}$, is defined as:

$$
\underline{B} * \psi \equiv-\frac{1}{2 m} \nabla^{2} \psi+U \psi
$$

and $*$ represents the inner product over the space of functions. The two operators, $\underline{A}$ and $\underline{B}$, have the properties:

$$
\begin{aligned}
& \bar{A}=-\underline{A} \\
& \bar{B}=\underline{B}
\end{aligned}
$$

The conjugate momentum, $p_{\psi}$, is given by:

$$
p_{\psi} \equiv \partial_{\dot{\psi}} \mathscr{L}=\underline{A} * \psi
$$

and the Euler-Lagrange equation is:

$$
\begin{aligned}
\dot{p_{\psi}} & =\partial_{\psi} \mathscr{L} \\
d_{t}(\underline{A} * \psi) & =\bar{A} * \dot{\psi}-2 \underline{B} * \psi \\
\underline{A} * \dot{\psi} & =-\underline{A} * \dot{\psi}-2 \underline{B} * \psi \\
\underline{A} * \dot{\psi} & =-\underline{B} * \psi \\
i \dot{\psi} & =-\frac{1}{2 m} \nabla^{2} \psi+U \psi
\end{aligned}
$$

which is the Schrödinger equation. The Hamiltonian is given by:

$$
\begin{aligned}
\mathscr{H} & =p_{\psi} * \dot{\psi}-\mathscr{L}=\dot{\psi} * \underline{A} * \psi-\dot{\psi} * \underline{A} * \psi+\psi * \underline{B} * \psi \\
& =\psi * \underline{B} * \psi=\int d^{3} x \Re\left[\psi^{*}\left(-\frac{1}{2 m} \nabla^{2}+U\right) \psi\right]
\end{aligned}
$$

However, we would like the mass to be represented by an anisotropic mass tensor. The classical theory of a particle with an anisotropic mass is given by the Lagrangian:

$$
\mathscr{L}=\frac{1}{2} v \cdot \underline{m} \cdot v-U(x)
$$


and the Euler-Lagrange equation:

$$
\begin{aligned}
p & =\partial_{v} \mathscr{L}=\underline{m} \cdot v \\
\dot{p} & =\underline{m} \cdot \dot{v}=-\partial_{x} U=F \\
F & =\underline{m} \cdot a
\end{aligned}
$$

We would like the center of mass of a wavepacket of our Schrödinger field to obey this classical equation. Therefore, we may modify the Schrödinger Lagrangian in the following way:

$$
\mathscr{L}=\int d^{3} x \Re\left[i \dot{\psi} \psi^{*}-\frac{1}{2}(\nabla \psi)^{*} \cdot \underline{m}^{-1} \cdot(\nabla \psi)-U \psi^{*} \psi\right]
$$

which gives rise to the anisotropic Schrödinger equation:

$$
i \dot{\psi}=-\frac{1}{2} \nabla \cdot \underline{m}^{-1} \cdot \nabla \psi+U \psi
$$

and the anisotropic Hamiltonian:

$$
\mathscr{H}=\int d^{3} x \Re\left[\psi^{*}\left(-\frac{1}{2} \nabla \cdot \underline{m}^{-1} \cdot \nabla+U\right) \psi\right]
$$

\section{F.5 The Elastic Lagrangian}

Following reference [78], we may derive the Lagrangian for an elastic body in the limit of small distortions. It is assumed that the elastic body has some equilibrium reference configuration, in which each point on the body may be labeled with its vector position, $x$. When the body is deformed, each point, $x$, moves to a new location, $y(x)=x+u(x)$, where $u(x)$ is the displacement of the point, $x$. Therefore, the displacement field, $u(x)$, may be used to define the deformation of the elastic body.

The displacement field will cause the local neighborhood of a point, $x$, to become stretched and rotated. The tensor, $\underline{f}$, representing this stretch and rotation is given 
by the derivative of the new position, $y$, with respect to the old position, $x$ :

$$
\underline{f}=\underline{y}^{\grave{\nabla}}=\underline{1}+\underline{u} \underline{\bar{\nabla}}
$$

where 1 is the identity operator, and where the over and underbars on the vectors are equivalent to the column and row vectors of matrix algebra, respectively. Or, equivalently, in index notation:

$$
f_{j}^{i}=\partial^{i} y_{j}=\delta_{j}^{i}+\partial^{i} u_{j}
$$

We define the $\underline{h}$ tensor to be:

$$
\underline{h}=\underline{u} \frac{\dot{u}}{\nabla}
$$

such that $\underline{f}=\underline{1}+\underline{h}$. The local elastic potential energy density will depend on the local stretching, but not on the local rotation. Therefore, we define a tensor, $\underline{G}$, to be:

$$
\underline{G}=\bar{f} \underline{f}=(\underline{1}+\bar{h})(\underline{1}+\underline{h})=\underline{1}+\underline{h}+\bar{h}+\bar{h} \underline{h}
$$

This tensor is the square of the local stretch, and is independent of the local rotation. Next, if we assume that the deformation is small, we may linearize the theory by dropping terms of order $h^{2}$ :

$$
\underline{G}=\underline{1}+\underline{h}+\bar{h}
$$

This necessarily precludes the treatment of anharmonic phonon processes within this model. Because $\underline{G}$ is the square of the local stretch, we may define the strain tensor to be the deviation of the local stretch, $\sqrt{G}$, from unity:

$$
\underline{s}=\sqrt{\underline{G}}-\underline{1}=\sqrt{\underline{1}+\underline{h}+\bar{h}}-\underline{1}
$$

Therefore, the linearized strain tensor is equal to:

$$
\underline{s} \equiv \frac{1}{2}(\underline{h}+\bar{h})
$$


The strain tensor represents the deviation from the unstretched state, and it is symmetric by definition: $\bar{s}=\underline{s}$. The local elastic potential energy, $V$, is a function of $\underline{s}$. It is also minimized when the elastic body is in its undeformed state where $\underline{s}=0$. If $V$ is expanded as a power-series in $\underline{s}$, the lowest-order term is quadratic, and in the linearized theory all higher-order terms will be assumed to be negligible. Therefore, $V$ has the form:

$$
V=\frac{1}{2} \underline{s} \cdot \underline{\underline{K}} \cdot \underline{s}=\frac{1}{2} s_{i j} K^{i j k l} s_{k l}
$$

where $\underline{\underline{K}}$ is the elasticity tensor. Without loss of generality, the $\underline{\underline{K}}$ tensor may be assumed symmetric in its first two and last two indices, and under their exchange, which, in three dimensions, gives 21 independent components. Using this form of the potential energy, the linearized elastic Lagrangian is:

$$
\mathscr{L}=\int d^{3} x\left(\frac{1}{2} \rho \dot{u} \cdot \dot{u}-\frac{1}{2} \underline{s} \cdot \underline{\underline{K}} \cdot \underline{s}\right)
$$

where $\rho$ is the mass density of the material, and therefore $\frac{1}{2} \rho \dot{u} \cdot \dot{u}$ is the kinetic energy density.

As before, this Lagrangian may be rewritten in the language of generalized vectors:

$$
\mathscr{L}=\frac{1}{2} \dot{u} * \underline{A} * \dot{u}-\frac{1}{2} u * \underline{B} * u
$$

where the operator, $\underline{A}$, is defined as:

$$
\underline{A} * u \equiv \rho u
$$

and the operator, $\underline{B}$, is defined as:

$$
\underline{B} * u \equiv-(\underline{\underline{K}} \cdot \underline{s}) \cdot \dot{\nabla}=-(\underline{\underline{K}} \cdot(\underline{\nabla} \bar{u})) \cdot \dot{\nabla}
$$

or in index notation:

$$
\underline{B}^{i l} * u_{l}=-K^{i j k l} \partial_{j} \partial_{k} u_{l}
$$


The conjugate momentum, $p_{u}$, is:

$$
p_{u}=\partial_{\dot{u}} \mathscr{L}=\underline{A} * \dot{u}=\rho \dot{u}
$$

and is equal to the density of physical momentum in the elastic material. The EulerLagrange equation is:

$$
\begin{aligned}
\dot{p_{u}} & =\partial_{u} \mathscr{L} \\
\rho \ddot{u} & =-\underline{B} * u \\
\rho \ddot{u} & =(\underline{\underline{K}} \cdot \underline{s}) \cdot \dot{\nabla} \\
\rho \ddot{u} & =\underline{\dot{\sigma}} \cdot \dot{\nabla}
\end{aligned}
$$

where the tensor, $\underline{\sigma}$, is defined to be:

$$
\underline{\sigma} \equiv \underline{\underline{K}} \cdot \underline{s}
$$

The left-hand-side of the Euler-Lagrange equation is the rate of change of physical momentum density, which, by Newton's second law, is equal to the force density, which is the divergence of the stress tensor. Therefore, $\underline{\sigma}$, must be the stress tensor.

The Hamiltonian is given by:

$$
\begin{aligned}
\mathscr{H} & =p_{u} * \dot{u}-\mathscr{L}=\dot{u} * \underline{A} * \dot{u}-\frac{1}{2} \dot{u} * \underline{A} * \dot{u}+\frac{1}{2} u * \underline{B} * u \\
& =\frac{1}{2} \dot{u} * \underline{A} * \dot{u}+\frac{1}{2} u * \underline{B} * u \\
& =\int d^{3} x\left(\frac{1}{2 \rho} p_{u} \cdot p_{u}+\frac{1}{2} \underline{s} \cdot \underline{\underline{K}} \cdot \underline{s}\right) \\
& =\int d^{3} x\left(\frac{1}{2 \rho} p_{u} \cdot p_{u}-\frac{1}{2} u \cdot(\underline{\underline{K}} \cdot \underline{s}) \cdot \dot{\nabla}\right)
\end{aligned}
$$

\section{F.6 The Electron-Phonon Interaction}

The Schrödinger field and the elastic displacement field described in the previous two sections may be used to model the electron and phonon systems in a semiconductor 
crystal. Both of these fields are based on a continuum, and therefore neglect the atomic nature of the crystal. However, for length scales much larger than the interatomic spacing, this model will be sufficient to capture the most prominent aspects of the electron-phonon interaction. ${ }^{1}$

Following reference [80], an interaction between the electron and phonon systems may be introduced into the model. As discussed in $§ 5.1$, the eigenstates of electrons traveling through a periodic crystal lattice are Bloch wavefunctions with energy vs. momentum functions which form distinct energy bands. As discussed in $\S 5.3$, the function, $\mathscr{E}(k)$, may be Taylor-expanded about its local minimum:

$$
\mathscr{E}(k)=\mathscr{E}_{0}+\frac{1}{2} k \cdot \underline{m}^{-1} \cdot k+\ldots
$$

where tensor, $\underline{m}$, is the effective mass tensor of the electron. The effective continuum wavefunction obeys the anisotropic Schrödinger equation:

$$
i \dot{\psi}=-\frac{1}{2} \nabla \cdot \underline{m}^{-1} \cdot \nabla \psi+\mathscr{E}_{0} \psi
$$

The energy vs. momentum function, $\mathscr{E}(k)$, for a particular band depends on the crystal structure, and it is therefore modified in the presence of a uniform crystal strain. If the strain is small, we may Taylor-expand the parameters, $\mathscr{E}_{0}$ and $\underline{m}$, with respect to the strain tensor, $\underline{s}$. However, as stated in [80], the effects of the shift in $\underline{m}$ will be negligable compared to the effects of the shift in $\mathscr{E}_{0}$ at low momentum.

Therefore, the dominant effect will be the shift in $\mathscr{E}_{0}$, which we may write as:

$$
\mathscr{E}_{0}(\underline{s})=U_{0}+\underline{X} \cdot \underline{s}+\ldots=U_{0}+X_{i j} s^{i j}+\ldots
$$

where the tensor, $\underline{X}$, is called the deformation potential. For small deformations, we may neglect all terms higher than first-order in $\underline{s}$.

To complete the model of electron-phonon interactions, we may return to the

\footnotetext{
${ }^{1}$ This model also neglects electron spin, although it could be added in a straightforward way by allowing the $\psi$ field to be a spinor rather than a complex scalar.
} 
anisotropic Schrödinger Lagrangian:

$$
\mathscr{L}=\int d^{3} x \Re\left[i \dot{\psi} \psi^{*}-\frac{1}{2}(\nabla \psi)^{*} \cdot \underline{m}^{-1} \cdot(\nabla \psi)-U \psi^{*} \psi\right]
$$

When the strain is not uniform, the effective mass and the energy minimum will vary with position in the crystal. This effect may be modeled by allowing $\underline{m}$ and $U$ in the Schrödinger Lagrangian to be functions of position. Neglecting the variations in $\underline{m}$, we may allow the potential, $U$, to be given by $U=\underline{X} \cdot \underline{s}^{2}$ This term leads to an interaction Lagrangian of the form:

$$
\mathscr{L}_{I}=-\int d^{3} x \underline{X} \cdot \underline{s} \psi^{*} \psi
$$

\section{F.7 The Continuum Model}

Using the results of the previous sections, we may describe a simple continuum model of electrons and phonons in a crystal with the following Lagrangian:

$$
\begin{aligned}
\mathscr{L} & =\mathscr{L}_{E}+\mathscr{L}_{P}+\mathscr{L}_{I} \\
\mathscr{L}_{E} & =\int d^{3} x \Re\left[i \dot{\psi} \psi^{*}-\frac{1}{2}(\nabla \psi)^{*} \cdot \underline{m}^{-1} \cdot(\nabla \psi)\right] \\
\mathscr{L}_{P} & =\int d^{3} x\left(\frac{1}{2} \rho \dot{u} \cdot \dot{u}-\frac{1}{2} \underline{s} \cdot \underline{\underline{K}} \cdot \underline{s}\right) \\
\mathscr{L}_{I} & =-\int d^{3} x \underline{X} \cdot \underline{s} \psi^{*} \psi
\end{aligned}
$$

\footnotetext{
${ }^{2}$ We may drop the constant term, $U_{0}$, because it has no effect on the interaction.
} 
The corresponding Hamiltonian is:

$$
\begin{aligned}
\mathscr{H} & =\mathscr{H}_{E}+\mathscr{H}_{P}+\mathscr{H}_{I} \\
\mathscr{H}_{E} & =\int d^{3} x \Re\left[\psi^{*}\left(-\frac{1}{2} \nabla \cdot \underline{m}^{-1} \cdot \nabla\right) \psi\right] \\
\mathscr{H}_{P} & =\int d^{3} x\left(\frac{1}{2 \rho} p_{u} \cdot p_{u}-\frac{1}{2} u \cdot(\underline{\underline{K}} \cdot \underline{s}) \cdot \dot{\nabla}\right) \\
\mathscr{H}_{I} & =\int d^{3} x \underline{X} \cdot \underline{s} \psi^{*} \psi
\end{aligned}
$$

\section{F.8 Transformation to Fourier Space}

We may transform the problem to Fourier space by assuming the electron and phonon fields exist within a cubical box of volume $\mathscr{V}$ with periodic boundary conditions. The electron and phonon fields may be rewritten in terms of their complex Fourier components:

$$
\begin{aligned}
& \psi(x)=\sum_{k} \psi_{k} e^{i k \cdot x} \\
& u(x)=\sum_{k} u_{k} e^{i k \cdot x}
\end{aligned}
$$

where $u_{k}$ is a complex vector satisfying $u_{-k}=u_{k}^{*}$, and $u_{k}=u_{k}^{s} \mathrm{e}_{k}^{s}$, where $s$ is the polarization index, running from 1 to 3 , and $\mathrm{e}_{k}^{s}$ is a set of polarization vectors which are, in general, a function of the wavevector, $k$. These plane-waves are modes of their corresponding wave equations. For the electron field:

$$
\begin{aligned}
i \dot{\psi} & =-\frac{1}{2} \nabla \cdot \underline{m}^{-1} \cdot \nabla \psi \\
& =\frac{1}{2} k \cdot \underline{m}^{-1} \cdot k \psi=\mathscr{E}_{k} \psi
\end{aligned}
$$

Where $\mathscr{E}_{k}$ is defined to be the frequency of the $k$ electron mode. Therefore, the electron dispersion relation is:

$$
\mathscr{E}_{k}=\frac{1}{2} k \cdot \underline{m}^{-1} \cdot k
$$


For the phonon field:

$$
\begin{aligned}
\rho \ddot{u} & =(\underline{\underline{K}} \cdot \underline{s}) \cdot \dot{\nabla} \\
\rho \ddot{u^{i}} & =K^{i j k l} \partial_{j} \partial_{k} u_{l} \\
& =-K^{i}{ }_{j k l} k^{j} k^{k} u^{l}=-M_{l}^{i}(k) u^{l}
\end{aligned}
$$

For any value of $k$, there will be three phonon modes, corresponding to the three eigenvectors and eigenvalues of the symmetric tensor, $\underline{M}(k)$, which is itself a function of $k$. The polarization vectors, $\mathrm{e}_{k}^{s}$, may be chosen to be unit vectors directed along the principle axes of $\underline{M}(k)$. The eigenvalues of $\underline{M}(k)$ determine the frequencies of these three modes:

$$
\underline{M}(k) \cdot \mathrm{e}_{k}^{s}=\rho\left(\omega_{k}^{s}\right)^{2} \mathrm{e}_{k}^{s}
$$

Because $\underline{M}(k)$ is symmetric, both the eigenvalues and the eigenvectors will be real. The tensor, $\underline{M}(k)$, is also quadratic in $k$, which means that the dispersion relation for the phonons is linear, and all phonons have a constant ${ }^{3}$ speed of sound: $\omega_{k}^{s}=c_{k}^{s}|k|$. The speed of sound for each mode, $c_{k}^{s}$, is generally a function of the direction of $k$. In an isotropic medium, the speeds of sound are constant with respect to the direction of $k$.

Additionally, note that $\underline{M}(-k)=\underline{M}(k)$, which means phonon modes with opposite wavevectors will have the same eigenvalues, and also the same unit eigenvectors, up to an arbitrary choice of sign.

The terms in the Hamiltonian may now each be rewritten in terms of Fourier

\footnotetext{
${ }^{3}$ This is only true when the phonon wavelength is much larger than the inter-atomic spacing. As the phonon wavelength approaches the inter-atomic spacing, the dispersion relation becomes non-linear, and the speed of sound becomes a function of phonon momentum.
} 
modes:

$$
\begin{aligned}
\int d^{3} x p_{u} \cdot p_{u} & =\int d^{3} x \sum_{k_{1}, k_{2}} p_{k_{1}} \cdot p_{k_{2}} e^{i\left(k_{1}+k_{2}\right) \cdot x} \\
& =\sum_{k_{1}, k_{2}} p_{k_{1}} \cdot p_{k_{2}} \delta\left[k_{1}+k_{2}\right] \mathscr{V}=\sum_{k} p_{-k} \cdot p_{k} \mathscr{V} \\
& =\sum_{k} p_{k}^{*} \cdot p_{k} \mathscr{V}=\sum_{k, s} p_{k}^{s *} p_{k}^{s} \mathscr{V} \\
\int d^{3} x u \cdot(\underline{\underline{K}} \cdot \underline{s}) \cdot \underline{\nabla} & =\int d^{3} x \sum_{k_{1}, k_{2}, s} u_{k_{1}} \cdot \underline{M}\left(k_{2}\right) \cdot u_{k_{2}} e^{i\left(k_{1}+k_{2}\right) \cdot x} \\
& =\sum_{k} u_{k}^{*} \cdot \underline{M}(k) \cdot u_{k} \mathscr{V}=\sum_{k, s} \rho\left(\omega_{k}^{s}\right)^{2} u_{k}^{s *} u_{k}^{s} \mathscr{V} \\
\int d^{3} x \Re\left[\psi^{*}\left(-\frac{1}{2} \nabla \cdot \underline{m}^{-1} \cdot \nabla\right) \psi\right] & =\int d^{3} x \sum_{k_{1}, k_{2}} \Re\left[\psi_{k_{1}}^{\dagger} \mathscr{E}_{k_{2}} \psi_{k_{2}} e^{\left.i\left(k_{2}-k_{1}\right) \cdot x\right]}\right. \\
\qquad \mathscr{E}_{k} \psi_{k}^{\dagger} \psi_{k} \mathscr{V} &
\end{aligned}
$$

Therefore, in terms of Fourier modes, the full electron and phonon Hamiltonians are given by:

$$
\begin{aligned}
\mathscr{H}_{P} & =\sum_{k, s} \mathscr{V}\left(\frac{1}{2 \rho} p_{k}^{*} \cdot p_{k}+\frac{1}{2} u_{k}^{*} \cdot \underline{M}(k) \cdot u_{k}\right) \\
& =\sum_{k, s} \mathscr{V}\left(\frac{1}{2 \rho} p_{k}^{s *} p_{k}^{s}+\frac{1}{2} \rho\left(\omega_{k}^{s}\right)^{2} u_{k}^{s *} u_{k}^{s}\right) \\
\mathscr{H}_{E} & =\sum_{k} \mathscr{V} \mathscr{E}_{k} \psi_{k}^{\dagger} \psi_{k}
\end{aligned}
$$




\section{F.9 Canonical Quantization}

The field theory may be quantized by reinterpreting the mode amplitudes as operators, and introducing creation and annihilation operators. For the phonon field:

$$
\begin{aligned}
& u_{k}=\sqrt{\frac{1}{2 \rho \mathscr{V} \omega_{k}^{s}}}\left(a_{k}^{s} \mathrm{e}_{k}^{s}+a_{-k}^{s \dagger} \mathrm{e}_{-k}^{s}\right) \\
& p_{k}=-i \sqrt{\frac{\rho \omega_{k}^{s}}{2 \mathscr{V}}}\left(a_{k}^{s} \mathrm{e}_{k}^{s}-a_{-k}^{s \dagger} \mathrm{e}_{-k}^{s}\right)
\end{aligned}
$$

The phonon field is bosonic, which implies the creation and annihilation operators have bosonic commutation relations:

$$
\begin{gathered}
{\left[a_{k_{1}}^{s_{1}}, a_{k_{2}}^{s_{2} \dagger}\right]=\delta\left[k_{1}-k_{2}\right] \delta^{s_{1} s_{2}}} \\
{\left[a_{k_{1}}^{s_{1}}, a_{k_{2}}^{s_{2}}\right]=\left[a_{k_{1}}^{s_{1} \dagger}, a_{k_{2}}^{s_{2} \dagger}\right]=0}
\end{gathered}
$$

where $\delta[. .$.$] is the Kronecker delta function. The electron field may also be expressed$ in terms of creation and annihilation operators:

$$
\begin{aligned}
\psi_{k} & =\frac{1}{\sqrt{\mathscr{V}}} b_{k} \\
p_{k} & =\frac{i}{\sqrt{\mathscr{V}}} b_{-k}^{\dagger}
\end{aligned}
$$

The electron field is fermionic, which imples the creation and annihilation operators have fermionic anti-commutation relations:

$$
\begin{gathered}
\left\{b_{k_{1}}, b_{k_{2}}^{\dagger}\right\}=\delta\left[k_{1}-k_{2}\right] \\
\left\{b_{k_{1}}, b_{k_{2}}\right\}=\left\{b_{k_{1}}^{\dagger}, b_{k_{2}}^{\dagger}\right\}=0
\end{gathered}
$$

These commutation and anti-commutation relations give rise to the following commutation and anti-commutation relations for the field operators:

$$
\left[u(x), p_{u}(y)\right]=i \delta^{3}(x-y)
$$




$$
\left\{\psi(x), p_{\psi}(y)\right\}=i \delta^{3}(x-y)
$$

In terms of these creation and annihilation operators, the electron and phonon Hamiltonians are:

$$
\begin{aligned}
\mathscr{H}_{P} & =\sum_{k, s} \omega_{k}^{s}\left(a_{k}^{s \dagger} a_{k}^{s}+\frac{1}{2}\right) \\
\mathscr{H}_{E} & =\sum_{k} \mathscr{E}_{k} b_{k}^{\dagger} b_{k}
\end{aligned}
$$

Each mode of the phonon field behaves like a quantum harmonic oscillator, and may be populated by any non-negative integer number of phonons. Each mode of the electron field behaves like a quantum two-state system, and may only be populated by either 0 or 1 electrons.

\section{F.10 The Interaction Hamiltonian}

The interaction Hamiltonian may be written in terms of the creation and annihilation operators:

$$
\begin{aligned}
\mathscr{H}_{I} & =\int d^{3} x \nabla \cdot \underline{X} \cdot u \psi^{*} \psi \\
& =\int d^{3} x \sum_{q, k_{1}, k_{2}} i q \cdot \underline{X} \cdot u_{q} \psi_{k_{1}}^{\dagger} \psi_{k_{2}} e^{i\left(q-k_{1}+k_{2}\right) \cdot x} \\
& =\sum_{q, s, k_{1}, k_{2}} i q \cdot \underline{X} \cdot \frac{\left(a_{q}^{s} \mathrm{e}_{q}^{s}+a_{-q}^{s \dagger} \mathrm{e}_{-q}^{s}\right)}{\sqrt{2 \rho^{\mathscr{V}} \omega_{q}^{s}}} b_{k_{1}}^{\dagger} b_{k_{2}} \delta\left[q-k_{1}+k_{2}\right]
\end{aligned}
$$

This Hamiltonian may be written as the sum of two terms:

$$
\begin{aligned}
\mathscr{H}_{I}= & \sum_{q, s, k_{1}, k_{2}} \frac{i q \cdot \underline{X} \cdot \mathrm{e}_{q}^{s}}{\sqrt{2 \rho \mathscr{V} \omega_{q}^{s}}} a_{q}^{s} b_{k_{1}}^{\dagger} b_{k_{2}} \delta\left[q-k_{1}+k_{2}\right] \\
& -\sum_{q, s, k_{1}, k_{2}} \frac{i q \cdot \underline{X} \cdot \mathrm{e}_{q}^{s}}{\sqrt{2 \rho \mathscr{V} \omega_{q}^{s}}} a_{q}^{s \dagger} b_{k_{1}}^{\dagger} b_{k_{2}} \delta\left[-q-k_{1}+k_{2}\right]
\end{aligned}
$$


The first term represents phonon absorption by an electron, and the second term represents phonon emission by an electron.

\section{F.11 Phonon Emission and Absorption Rates}

In this section, the rate of phonon emission or absorption by an electron in a momentum eigenstate is calculated, following the methods outlined in references [81] \& [72]. For clarity, electron momentum vectors will be designated by $k$, and phonon momentum vectors will be designated by $q$. The initial state is assumed to consist of a single electron with momentum, $k$, and a phonon background with mode occupation numbers, $n_{q}^{s}$ :

$$
|\psi(0)\rangle=\left|k ; n_{1}, n_{2}, n_{3}, \ldots\right\rangle
$$

This problem is best solved in the interaction picture, in which the time-evolution due to the free-particle Hamiltonian has been factored out. In this picture, the interaction Hamiltonian is time-dependant:

$$
\begin{aligned}
\mathscr{H}_{I}(t)= & \sum_{q, s, k_{1}, k_{2}} \frac{i q \cdot \underline{X} \cdot \mathrm{e}_{q}^{s}}{\sqrt{2 \rho^{\mathscr{V}} \omega_{q}^{s}}} a_{q}^{s} b_{k_{1}}^{\dagger} b_{k_{2}} \delta\left[q-k_{1}+k_{2}\right] e^{i\left(-\omega_{q}+\mathscr{E}_{k_{1}}-\mathscr{E}_{k_{2}}\right) t} \\
& -\sum_{q, s, k_{1}, k_{2}} \frac{i q \cdot \underline{X} \cdot \mathrm{e}_{q}^{s}}{\sqrt{2 \rho \mathscr{V} \omega_{q}^{s}}} a_{q}^{s \dagger} b_{k_{1}}^{\dagger} b_{k_{2}} \delta\left[-q-k_{1}+k_{2}\right] e^{i\left(\omega_{q}+\mathscr{E}_{k_{1}}-\mathscr{E}_{k_{2}}\right) t}(
\end{aligned}
$$

The Schrödinger equation in the interaction picture is:

$$
i \frac{d}{d t}|\psi(t)\rangle=\mathscr{H}_{I}(t)|\psi(t)\rangle
$$

The interaction Hamiltonian allows the initial state to decay to a state consisting of an electron with a new momentum vector, plus or minus one phonon. For simplicity, we will focus only on single phonon emission or absorption at early times. 
In this case, the wavefunction is given approximately by:

$$
\begin{aligned}
|\psi(t)\rangle \approx \quad & \left|k ; n_{1}, n_{2}, n_{3}, \ldots\right\rangle \\
& +\sum_{q, s} \epsilon_{q}^{s}(t)\left|k-q ; \ldots, n_{q}^{s}+1, \ldots\right\rangle \\
& +\sum_{q, s} \alpha_{q}^{s}(t)\left|k+q ; \ldots, n_{q}^{s}-1, \ldots\right\rangle
\end{aligned}
$$

The Schrödinger equation gives the following set of first-order differential equations for the $s$ and $\alpha$ coefficients:

$$
\begin{aligned}
\dot{\epsilon}_{q}^{s} & \approx-i \sqrt{n_{q}^{s}+1} \frac{q \cdot \underline{X} \cdot \mathrm{e}_{q}^{s}}{\sqrt{2 \rho^{\mathscr{V}} \omega_{q}^{s}}} e^{i\left(\omega_{q}+\mathscr{E}_{k-q}-\mathscr{E}_{k}\right) t} \\
\dot{\alpha}_{q}^{s} & \approx i \sqrt{n_{q}^{s}} \frac{q \cdot \underline{X} \cdot \mathrm{e}_{q}^{s}}{\sqrt{2 \rho^{\mathscr{V}} \omega_{q}^{s}}} e^{i\left(-\omega_{q}+\mathscr{E}_{k+q}-\mathscr{E}_{k}\right) t}
\end{aligned}
$$

This set of differential equations is easily solved by the following integral:

$$
\int_{0}^{t} d t^{\prime} e^{i \omega t^{\prime}}=\frac{e^{i \omega t}-1}{i \omega} \equiv f(\omega ; t)
$$

The function, $f$, has the following important properties:

$$
\begin{aligned}
& f(\omega ; t) f^{*}(\omega ; t)=\frac{2-2 \cos (\omega t)}{\omega^{2}} \\
& \frac{d}{d t}\left(f^{*} f\right)=\frac{2 \sin (\omega t)}{\omega} \approx 2 \pi \delta(\omega)
\end{aligned}
$$

The function $2 \sin (\omega t) / \omega$ has a width of approximately $1 / t$ and for values of $\omega$ much larger than this it may be approximated by a delta function. The set of $\epsilon$ and $\alpha$ coefficients is given by:

$$
\begin{aligned}
\epsilon_{q}^{s}(t) & =-i \sqrt{n_{q}^{s}+1} \frac{q \cdot \underline{X} \cdot \mathrm{e}_{q}^{s}}{\sqrt{2 \rho^{\mathscr{V}} \omega_{q}^{s}}} f\left(\omega_{q}+\mathscr{E}_{k-q}-\mathscr{E}_{k} ; t\right) \\
\alpha_{q}^{s}(t) & =i \sqrt{n_{q}^{s}} \frac{q \cdot \underline{X} \cdot \mathrm{e}_{q}^{s}}{\sqrt{2 \rho^{\mathscr{V}} \omega_{q}^{s}}} f\left(-\omega_{q}+\mathscr{E}_{k+q}-\mathscr{E}_{k} ; t\right)
\end{aligned}
$$


The probability of phonon emission into the mode, $q$, with polarization, $s$, is:

$$
p_{E q}^{s}=\epsilon_{q}^{s *} \epsilon_{q}^{s}=\left(n_{q}^{s}+1\right) \frac{\left(q \cdot \underline{X} \cdot \mathrm{e}_{q}^{s}\right)^{2}}{2 \rho \mathscr{V} \omega_{q}^{s}} f^{*} f
$$

The probability of phonon absorption from the mode, $q$, with polarization, $s$, is:

$$
p_{A q}^{s}=\alpha_{q}^{s *} \alpha_{q}^{s}=\left(n_{q}^{s}\right) \frac{\left(q \cdot \underline{X} \cdot \mathrm{e}_{q}^{s}\right)^{2}}{2 \rho \mathscr{V} \omega_{q}^{s}} f^{*} f
$$

The rates of change of these probabilities are:

$$
\begin{aligned}
& \dot{p}_{E q}^{s} \approx\left(n_{q}^{s}+1\right) \frac{\left(q \cdot \underline{X} \cdot \mathrm{e}_{q}^{s}\right)^{2}}{2 \rho \mathscr{V} \omega_{q}^{s}} 2 \pi \delta\left(\omega_{q}^{s}+\mathscr{E}_{k-q}-\mathscr{E}_{k}\right) \\
& \dot{p}_{A q}^{s} \approx\left(n_{q}^{s}\right) \frac{\left(q \cdot \underline{X} \cdot \mathrm{e}_{q}^{s}\right)^{2}}{2 \rho \mathscr{V} \omega_{q}^{s}} 2 \pi \delta\left(-\omega_{q}^{s}+\mathscr{E}_{k+q}-\mathscr{E}_{k}\right)
\end{aligned}
$$

The probability that a phonon has been emitted or absorbed is the sum over the probabilities of emission or absorption into each phonon mode:

$$
\begin{aligned}
& P_{E}=\sum_{q, s} p_{E q}^{s} \\
& P_{A}=\sum_{q, s} p_{A q}^{s}
\end{aligned}
$$

The rates at which these probabilities increase are defined to be the scattering rates for both emission and absorption:

$$
\begin{aligned}
\Gamma_{E} & \equiv \dot{P}_{E}=\sum_{q, s} \dot{p}_{E q}^{s}=\sum_{q, s}\left(n_{q}^{s}+1\right) \frac{\left(q \cdot \underline{X} \cdot \mathrm{e}_{q}^{s}\right)^{2}}{2 \rho \mathscr{V} \omega_{q}^{s}} 2 \pi \delta\left(\omega_{q}^{s}+\mathscr{E}_{k-q}-\mathscr{E}_{k}\right) \\
\Gamma_{A} & \equiv \dot{P}_{A}=\sum_{q, s} \dot{p}_{A q}^{s}=\sum_{q, s}\left(n_{q}^{s}\right) \frac{\left(q \cdot \underline{X} \cdot \mathrm{e}_{q}^{s}\right)^{2}}{2 \rho \mathscr{V} \omega_{q}^{s}} 2 \pi \delta\left(-\omega_{q}^{s}+\mathscr{E}_{k+q}-\mathscr{E}_{k}\right)
\end{aligned}
$$

These sums over modes may be approximated by integrals over $q$-space when the 
volume, $\mathscr{V}$, is large. The volume element in $q$-space is given by: $\Delta q^{3} /(2 \pi)^{3}=1 / \mathscr{V}$.

$$
\begin{aligned}
\Gamma_{E} & =\sum_{s} \int \frac{d^{3} q}{(2 \pi)^{3}}\left(n_{q}^{s}+1\right) \frac{\left(q \cdot \underline{X} \cdot \mathrm{e}_{q}^{s}\right)^{2}}{2 \rho \omega_{q}^{s}} 2 \pi \delta\left(\omega_{q}^{s}+\mathscr{E}_{k-q}-\mathscr{E}_{k}\right) \\
\Gamma_{A} & =\sum_{s} \int \frac{d^{3} q}{(2 \pi)^{3}}\left(n_{q}^{s}\right) \frac{\left(q \cdot \underline{X} \cdot \mathrm{e}_{q}^{s}\right)^{2}}{2 \rho \omega_{q}^{s}} 2 \pi \delta\left(-\omega_{q}^{s}+\mathscr{E}_{k+q}-\mathscr{E}_{k}\right)
\end{aligned}
$$

\section{F.12 Differential Intra-Valley Scattering Rates}

The scattering rates for both emission and absorption may be written as integrals over both the magnitude and solid angle of the $q$ vector using the relation: $d^{3} q=|q|^{2} d \Omega d|q|$ :

$$
\begin{aligned}
\Gamma_{E} & =\sum_{s} \int \frac{|q|^{2} d \Omega d|q|}{(2 \pi)^{3}}\left(n_{q}^{s}+1\right) \frac{|q|^{2}}{|q|} \frac{\left(\hat{q} \cdot \underline{X} \cdot \mathrm{e}_{q}^{s}\right)^{2}}{2 \rho c_{q}^{s}} 2 \pi \delta\left(\omega_{q}^{s}+\mathscr{E}_{k-q}-\mathscr{E}_{k}\right) \\
& =\sum_{s} \int \frac{d \Omega d|q|}{(2 \pi)^{2}}\left(n_{q}^{s}+1\right)|q|^{3} \frac{\left(\hat{q} \cdot \underline{X} \cdot \mathrm{e}_{q}^{s}\right)^{2}}{2 \rho c_{q}^{s}} \delta\left(\omega_{q}^{s}+\mathscr{E}_{k-q}-\mathscr{E}_{k}\right) \\
\Gamma_{A} & =\sum_{s} \int \frac{|q|^{2} d \Omega d|q|}{(2 \pi)^{3}}\left(n_{q}^{s}\right) \frac{|q|^{2}}{|q|} \frac{\left(\hat{q} \cdot \underline{X} \cdot \mathrm{e}_{q}^{s}\right)^{2}}{2 \rho c_{q}^{s}} 2 \pi \delta\left(-\omega_{q}^{s}+\mathscr{E}_{k+q}-\mathscr{E}_{k}\right) \\
& =\sum_{s} \int \frac{d \Omega d|q|}{(2 \pi)^{2}}\left(n_{q}^{s}\right)|q|^{3} \frac{\left(\hat{q} \cdot \underline{X} \cdot \mathrm{e}_{q}^{s}\right)^{2}}{2 \rho c_{q}^{s}} \delta\left(-\omega_{q}^{s}+\mathscr{E}_{k+q}-\mathscr{E}_{k}\right)
\end{aligned}
$$

The integral over $|q|$ may be performed using the identity:

$$
\int d x f(x) \delta(g(x))=\int d g\left(\frac{d x}{d g}\right) f(x(g)) \delta(g)=\sum_{i} \frac{f\left(x_{i}\right)}{\left|g^{\prime}\left(x_{i}\right)\right|}
$$

where $x_{i}$ is the $i$ th zero of $g(x)$. For emission, $g(|q|)$ is:

$$
\begin{aligned}
g_{E}(|q|) & =\omega_{q}^{s}+\mathscr{E}_{k-q}-\mathscr{E}_{k} \\
& =|q| c_{q}^{s}+\frac{1}{2}(k-q) \cdot \underline{m}^{-1} \cdot(k-q)-\frac{1}{2} k \cdot \underline{m}^{-1} \cdot k \\
& =|q| c_{q}^{s}+\frac{1}{2}|q|^{2} \hat{q} \cdot \underline{m}^{-1} \cdot \hat{q}-|q| \hat{q} \cdot \underline{m}^{-1} \cdot k \\
& =|q|\left(c_{q}^{s}-\hat{q} \cdot v+\frac{1}{2}|q| \hat{q} \cdot \underline{m}^{-1} \cdot \hat{q}\right)
\end{aligned}
$$


Where $v$ is the electron velocity, $v=\underline{m}^{-1} \cdot k$. The function $g_{E}(|q|)$ is zero at:

$$
|q|=0 \quad \text { or } \quad|q|=2\left(\hat{q} \cdot \underline{m}^{-1} \cdot \hat{q}\right)^{-1}\left(\hat{q} \cdot v-c_{q}^{s}\right)
$$

Its derivative is:

$$
g_{E}^{\prime}(|q|)=c_{q}^{s}-\hat{q} \cdot v+|q| \hat{q} \cdot \underline{m}^{-1} \cdot \hat{q}
$$

At the point where $|q|=2\left(\hat{q} \cdot \underline{m}^{-1} \cdot \hat{q}\right)^{-1}\left(\hat{q} \cdot v-c_{q}^{s}\right)$ :

$$
g_{E}^{\prime}(|q|)=\hat{q} \cdot v-c_{q}^{s}
$$

Likewise, for absorption, the function $g(|q|)$ is:

$$
\begin{aligned}
g_{A}(|q|) & =-\omega_{q}^{s}+\mathscr{E}_{k+q}-\mathscr{E}_{k} \\
& =-|q| c_{q}^{s}+\frac{1}{2}(k+q) \cdot \underline{m}^{-1} \cdot(k+q)-\frac{1}{2} k \cdot \underline{m}^{-1} \cdot k \\
& =-|q| c_{q}^{s}+\frac{1}{2}|q|^{2} \hat{q} \cdot \underline{m}^{-1} \cdot \hat{q}+|q| \hat{q} \cdot \underline{m}^{-1} k \\
& =|q|\left(\hat{q} \cdot v-c_{q}^{s}+\frac{1}{2}|q| \hat{q} \cdot \underline{m}^{-1} \cdot \hat{q}\right)
\end{aligned}
$$

The function $g_{A}(|q|)$ is zero at:

$$
|q|=0 \quad \text { or } \quad|q|=2\left(\hat{q} \cdot \underline{m}^{-1} \cdot \hat{q}\right)^{-1}\left(c_{q}^{s}-\hat{q} \cdot v\right)
$$

Its derivative is:

$$
g_{A}^{\prime}(|q|)=\hat{q} \cdot v-c_{q}^{s}+|q| \hat{q} \cdot \underline{m}^{-1} \cdot \hat{q}
$$

At the point where $|q|=2\left(\hat{q} \cdot \underline{m}^{-1} \cdot \hat{q}\right)^{-1}\left(c_{q}^{s}-\hat{q} \cdot v\right)$ :

$$
g_{A}^{\prime}(|q|)=c_{q}^{s}-\hat{q} \cdot v
$$


The functions, $g_{E}$ and $g_{A}$, may be used to perform the integrals over $|q|$ :

$$
\begin{aligned}
\Gamma_{E} & =\sum_{s} \int \frac{d \Omega d|q|}{(2 \pi)^{2}}\left(n_{q}^{s}+1\right)|q|^{3} \frac{\left(\hat{q} \cdot \underline{X} \cdot \mathrm{e}_{q}^{s}\right)^{2}}{2 \rho c_{q}^{s}} \delta\left(g_{E}(|q|)\right) \\
& =\left.\sum_{s} \int \frac{d \Omega}{(2 \pi)^{2}}\left(n_{q}^{s}+1\right)|q|^{3} \frac{\left(\hat{q} \cdot \underline{X} \cdot \mathrm{e}_{q}^{s}\right)^{2}}{2 \rho c_{q}^{s} g_{E}^{\prime}(|q|)}\right|_{|q|=2\left(\hat{q} \cdot \underline{m}^{-1} \cdot \hat{q}\right)^{-1}\left(\hat{q} \cdot v-c_{q}^{s}\right)} \\
& =\sum_{s} \int \frac{d \Omega}{\pi^{2}}\left(n_{q}^{s}+1\right)\left(\hat{q} \cdot \underline{m^{-1}} \cdot \hat{q}\right)^{-3}\left(\hat{q} \cdot v-c_{q}^{s}\right)^{2} \frac{\left(\hat{q} \cdot \underline{X} \cdot \mathrm{e}_{q}^{s}\right)^{2}}{\rho c_{q}^{s}} \Theta\left(\hat{q} \cdot v-\left(\mathbb{E}_{q}^{s}\right) 103\right) \\
\Gamma_{A} & =\sum_{s} \int \frac{d \Omega d|q|}{(2 \pi)^{2}}\left(n_{q}^{s}\right)|q|^{3} \frac{\left(\hat{q} \cdot \underline{X} \cdot \mathrm{e}_{q}^{s}\right)^{2}}{2 \rho c_{q}^{s}} \delta\left(g_{A}(|q|)\right) \\
& =\left.\sum_{s} \int \frac{d \Omega}{(2 \pi)^{2}}\left(n_{q}^{s}\right)|q|^{3} \frac{\left(\hat{q} \cdot \underline{X} \cdot \mathrm{e}_{q}^{s}\right)^{2}}{2 \rho c_{q}^{s} g_{A}^{\prime}(|q|)}\right|_{|q|=2\left(\hat{q} \cdot \underline{m^{-1}} \cdot \hat{q}\right)^{-1}\left(c_{q}^{s}-\hat{q} \cdot v\right)} \\
& =\sum_{s} \int \frac{d \Omega}{\pi^{2}}\left(n_{q}^{s}\right)\left(\hat{q} \cdot \underline{m}^{-1} \cdot \hat{q}\right)^{-3}\left(c_{q}^{s}-\hat{q} \cdot v\right)^{2} \frac{\left(\hat{q} \cdot \underline{X} \cdot \mathrm{e}_{q}^{s}\right)^{2}}{\rho c_{q}^{s}} \Theta\left(c_{q}^{s}-\hat{q} \cdot v\right)(\mathrm{F} .104)
\end{aligned}
$$

Therefore, the differential scattering rates are given by:

$$
\begin{aligned}
\frac{d \Gamma_{E}^{s}}{d \Omega} & =\left(n_{q}^{s}+1\right)\left(\hat{q} \cdot \underline{m}^{-1} \cdot \hat{q}\right)^{-3}\left(\hat{q} \cdot v-c_{q}^{s}\right)^{2} \frac{\left(\hat{q} \cdot \underline{X} \cdot \mathrm{e}_{q}^{s}\right)^{2}}{\pi^{2} \rho c_{q}^{s}} \Theta\left(\hat{q} \cdot v-c_{q}^{s}\right) \\
\frac{d \Gamma_{A}^{s}}{d \Omega} & =\left(n_{q}^{s}\right)\left(\hat{q} \cdot \underline{m}^{-1} \cdot \hat{q}\right)^{-3}\left(c_{q}^{s}-\hat{q} \cdot v\right)^{2} \frac{\left(\hat{q} \cdot \underline{X} \cdot \mathrm{e}_{q}^{s}\right)^{2}}{\pi^{2} \rho c_{q}^{s}} \Theta\left(c_{q}^{s}-\hat{q} \cdot v\right)
\end{aligned}
$$

\section{F.13 Isotropic Intra-Valley Scattering Rates}

In an isotropic crystal, the mass tensor, $\underline{m}$, and deformation potential, $\underline{X}$, are both proportional to the identity tensor: $\underline{m}=m, \underline{X}=X$. In addition, the transverse and longitudinal phonon modes have isotropic speeds of sound, and their polarization vectors are perpendicular and parallel to the phonon momentum vector, respectively.

Therefore, in an isotropic crystal:

$$
\hat{q} \cdot \underline{X} \cdot \mathrm{e}_{q}^{s}=X \hat{q} \cdot \mathrm{e}_{q}^{s}=\left\{\begin{array}{l}
X \quad: s=\text { Longitudinal } \\
0 \quad: s=\text { Transverse }
\end{array}\right.
$$


This implies that only longitudinal phonons are emitted or absorbed in an isotropic crystal. The scattering rates are:

$$
\begin{aligned}
& \Gamma_{E}=\int d \Omega\left(n_{q}^{s}+1\right) m^{3}\left(\hat{q} \cdot v-c_{l}\right)^{2} \frac{X^{2}}{\pi^{2} \rho c_{l}} \Theta\left(\hat{q} \cdot v-c_{l}\right) \\
& \Gamma_{A}=\int d \Omega\left(n_{q}^{s}\right) m^{3}\left(c_{l}-\hat{q} \cdot v\right)^{2} \frac{X^{2}}{\pi^{2} \rho c_{l}} \Theta\left(c_{l}-\hat{q} \cdot v\right)
\end{aligned}
$$

where $c_{l}$ is the longitudinal speed of sound. Let us assume that the phonon system is in thermal equilibrium at temperature, $T=1 / \beta$. In an integral over $q$-space, we can assume that each volume element, $d^{3} q$, contains a large number of modes, each with a random population number, $n_{q}^{s}$, with the frequency of occurence each value of $n_{q}^{s}$ given by the Boltzmann Distribution. Therefore, any such integral which contains the mode population number, $n_{q}^{s}$, multiplied by a slowly-varying function of $q$ can be approximated by replacing $n_{q}^{s}$ with its thermal average, $\left\langle n_{q}^{s}\right\rangle$ :

$$
\left\langle n_{q}^{s}\right\rangle=\frac{1}{e^{\beta \omega_{q}^{s}}-1}=\frac{1}{e^{\beta|q| c_{q}^{s}}-1}
$$

For temperatures much higher than the phonon energies, the thermally averaged mode occupation number may be approximated by:

$$
\left\langle n_{q}^{s}\right\rangle \approx \frac{1}{\beta|q| c_{q}^{s}}=\frac{T}{|q| c_{q}^{s}} \gg 1
$$




\section{F.14 The High Temperature Limit}

In the high temperature limit, the scattering rates are approximately given by:

$$
\begin{aligned}
& \Gamma_{E}=\int d \Omega \frac{T}{|q| c_{l}} m^{3}\left(\hat{q} \cdot v-c_{l}\right)^{2} \frac{X^{2}}{\pi^{2} \rho c_{l}} \Theta\left(\hat{q} \cdot v-c_{l}\right) \\
& =\int d \Omega \frac{m^{2} T X^{2}}{2 \pi^{2} \rho c_{l}^{2}}\left(\hat{q} \cdot v-c_{l}\right) \Theta\left(\hat{q} \cdot v-c_{l}\right) \\
& =\frac{m^{2} T X^{2}}{2 \pi^{2} \rho c_{l}^{2}} \int \sin \theta d \theta d \phi\left(v \cos \theta-c_{l}\right) \Theta\left(v \cos \theta-c_{l}\right) \\
& =\frac{m^{2} T X^{2}}{2 \pi^{2} \rho c_{l}^{2}} \int_{-1}^{1} d x \int_{0}^{2 \pi} d \phi\left(v x-c_{l}\right) \Theta\left(v x-c_{l}\right) \\
& =\frac{m^{2} T X^{2}}{\pi \rho c_{l}^{2}} \int_{c_{l} / v}^{1} d x\left(v x-c_{l}\right) \\
& =\frac{m^{2} T X^{2}\left(v-c_{l}\right)^{2}}{2 \pi \rho c_{l}^{2} v} \Theta\left(v-c_{l}\right) \\
& \Gamma_{A}=\int d \Omega \frac{T}{|q| c_{l}} m^{3}\left(c_{l}-\hat{q} \cdot v\right)^{2} \frac{X^{2}}{\pi^{2} \rho c_{l}} \Theta\left(c_{l}-\hat{q} \cdot v\right) \\
& =\int d \Omega \frac{m^{2} T X^{2}}{2 \pi^{2} \rho c_{l}^{2}}\left(c_{l}-\hat{q} \cdot v\right) \Theta\left(c_{l}-\hat{q} \cdot v\right) \\
& =\frac{m^{2} T X^{2}}{2 \pi^{2} \rho c_{l}^{2}} \int \sin \theta d \theta d \phi\left(c_{l}-v \cos \theta\right) \Theta\left(c_{l}-v \cos \theta\right) \\
& =\frac{m^{2} T X^{2}}{2 \pi^{2} \rho c_{l}^{2}} \int_{-1}^{1} d x \int_{0}^{2 \pi} d \phi\left(c_{l}-v x\right) \Theta\left(c_{l}-v x\right) \\
& =\frac{m^{2} T X^{2}}{\pi \rho c_{l}^{2}} \int_{-1}^{\min \left(1, c_{l} / v\right)} d x\left(c_{l}-v x\right) \\
& = \begin{cases}\frac{2 m^{2} T X^{2}}{\pi \rho c_{l}} & \text { for } v<c_{l} \\
\frac{m^{2} T X^{2}\left(c_{l}+v\right)^{2}}{2 \pi \rho c_{l}^{2} v} & \text { for } v \geq c_{l}\end{cases}
\end{aligned}
$$




\section{F.15 The Low Temperature Limit}

In the low temperature limit, the thermally averaged phonon population number approaches zero. Therefore, as $T \rightarrow 0$, the phonon absorption rate will also approach zero. The phonon emission rate will be:

$$
\begin{aligned}
\Gamma_{E} & =\int d \Omega m^{3}\left(\hat{q} \cdot v-c_{l}\right)^{2} \frac{X^{2}}{\pi^{2} \rho c_{l}} \Theta\left(\hat{q} \cdot v-c_{l}\right) \\
& =\frac{m^{3} X^{2}}{\pi^{2} \rho c_{l}} \int \sin \theta d \theta d \phi\left(v \cos \theta-c_{l}\right)^{2} \Theta\left(v \cos \theta-c_{l}\right) \\
& =\frac{2 m^{3} X^{2}}{\pi \rho c_{l}} \int_{c_{l} / v}^{1} d x\left(v x-c_{l}\right)^{2} \\
& =\frac{2 m^{3} X^{2}\left(v-c_{l}\right)^{3}}{3 \pi \rho c_{l} v} \Theta\left(v-c_{l}\right)
\end{aligned}
$$




\section{Appendix G}

\section{Experimental Setup - Supplemental Material}

G.1 Mechanical Drawings

Copper Baseplate Layout for the Charge Transport Experiment

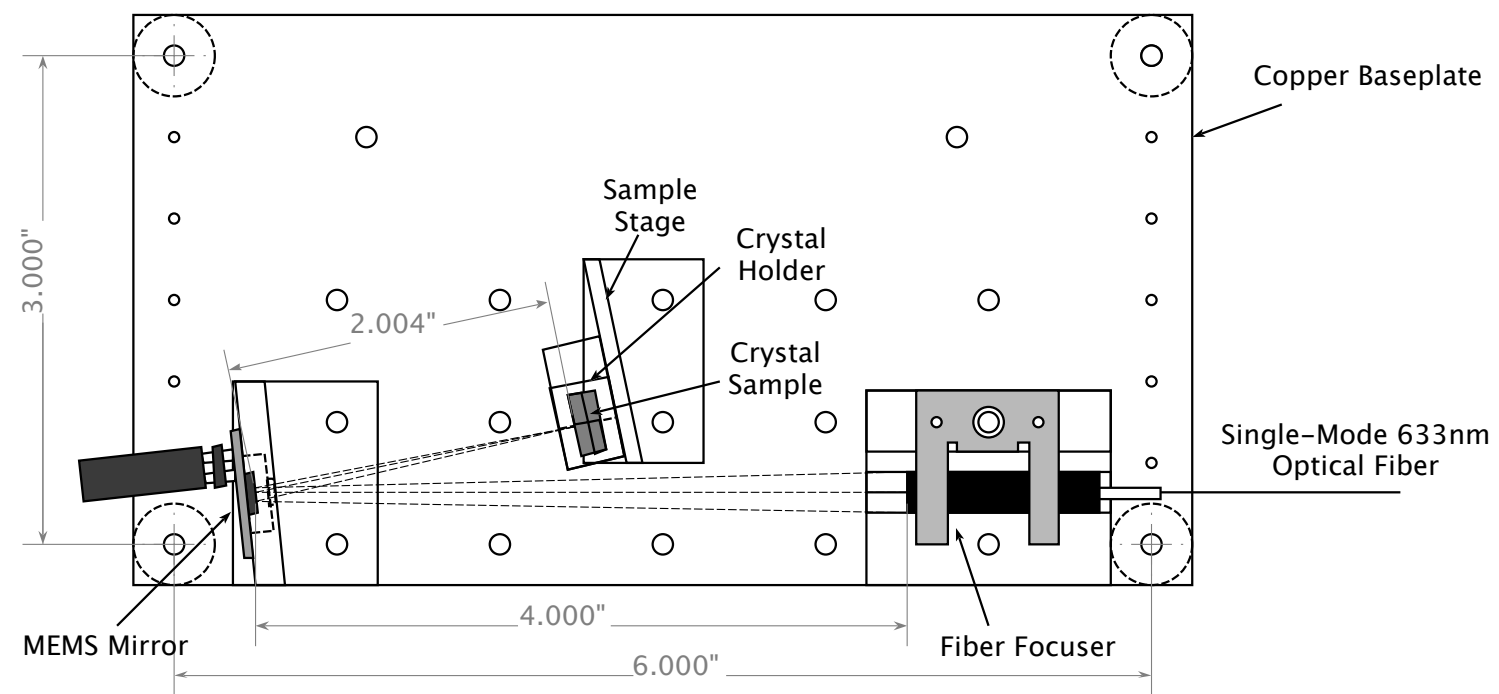

Figure G.1: Layout for the copper baseplate of the experimental payload. 
Fiber Focuser Holder:
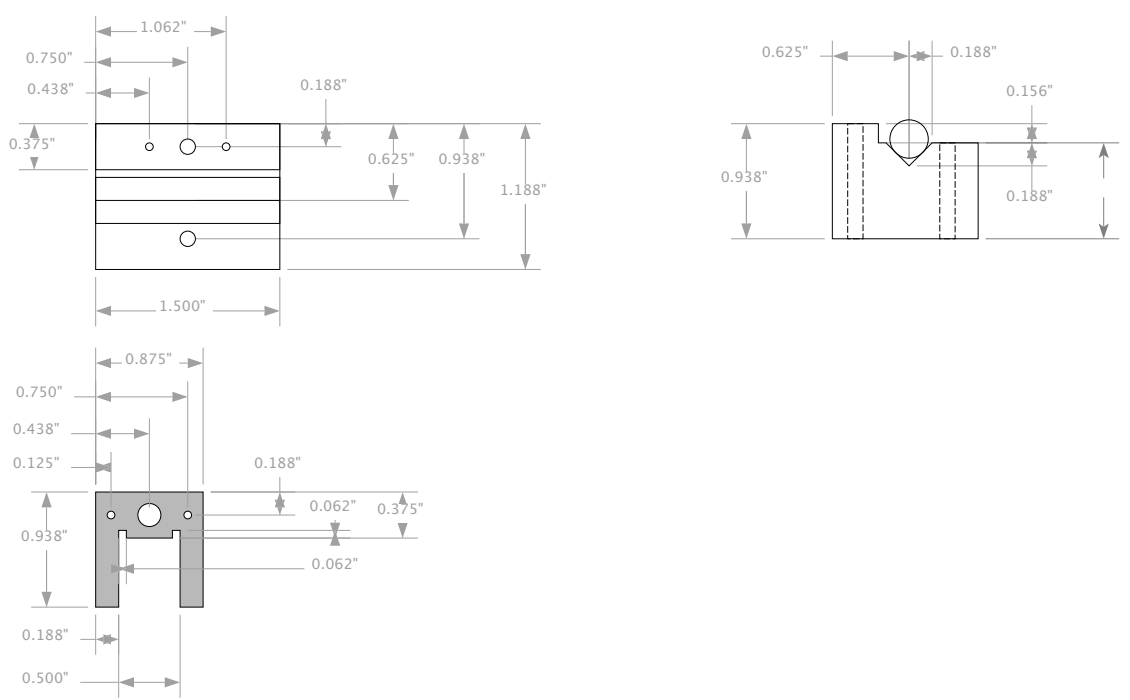

Figure G.2: Design of fiber focuser holder.

\section{Mirror Holder:}
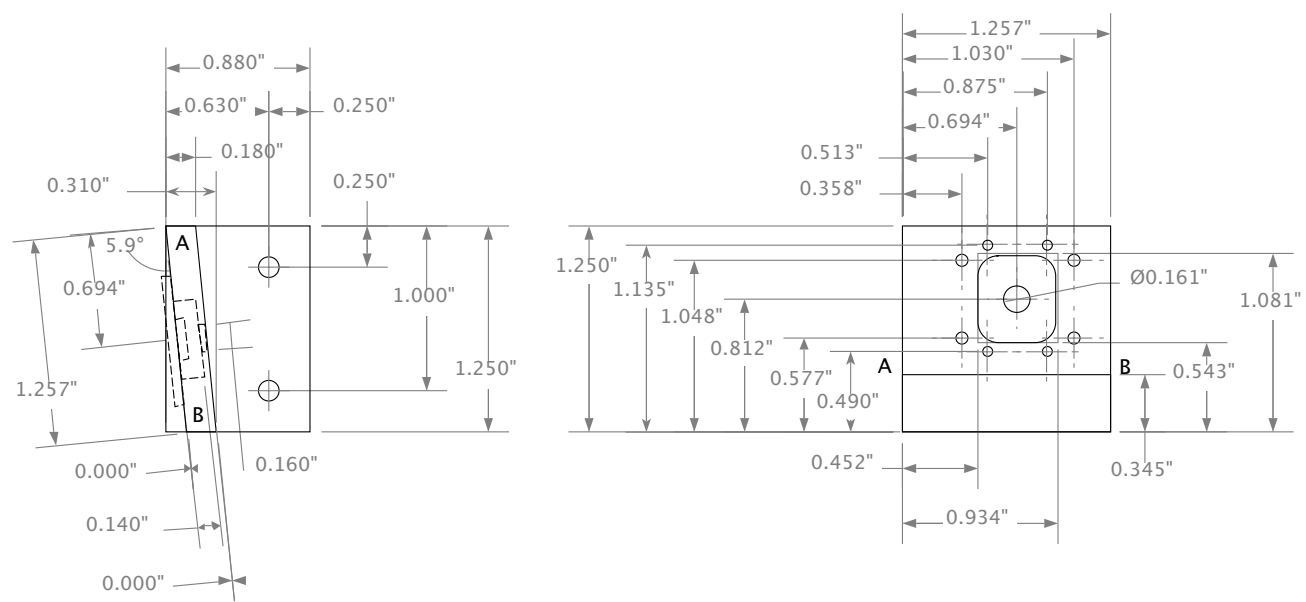

Figure G.3: Design of mirror holder. 


\section{Sample Stage:}
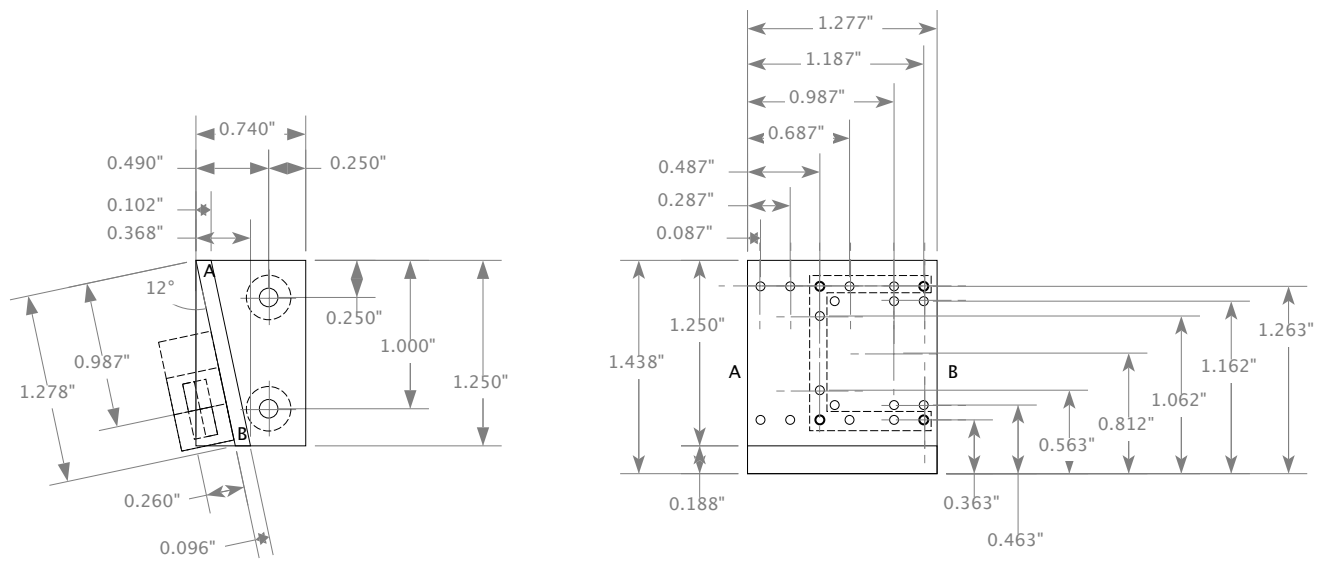

Figure G.4: Design of sample stage.
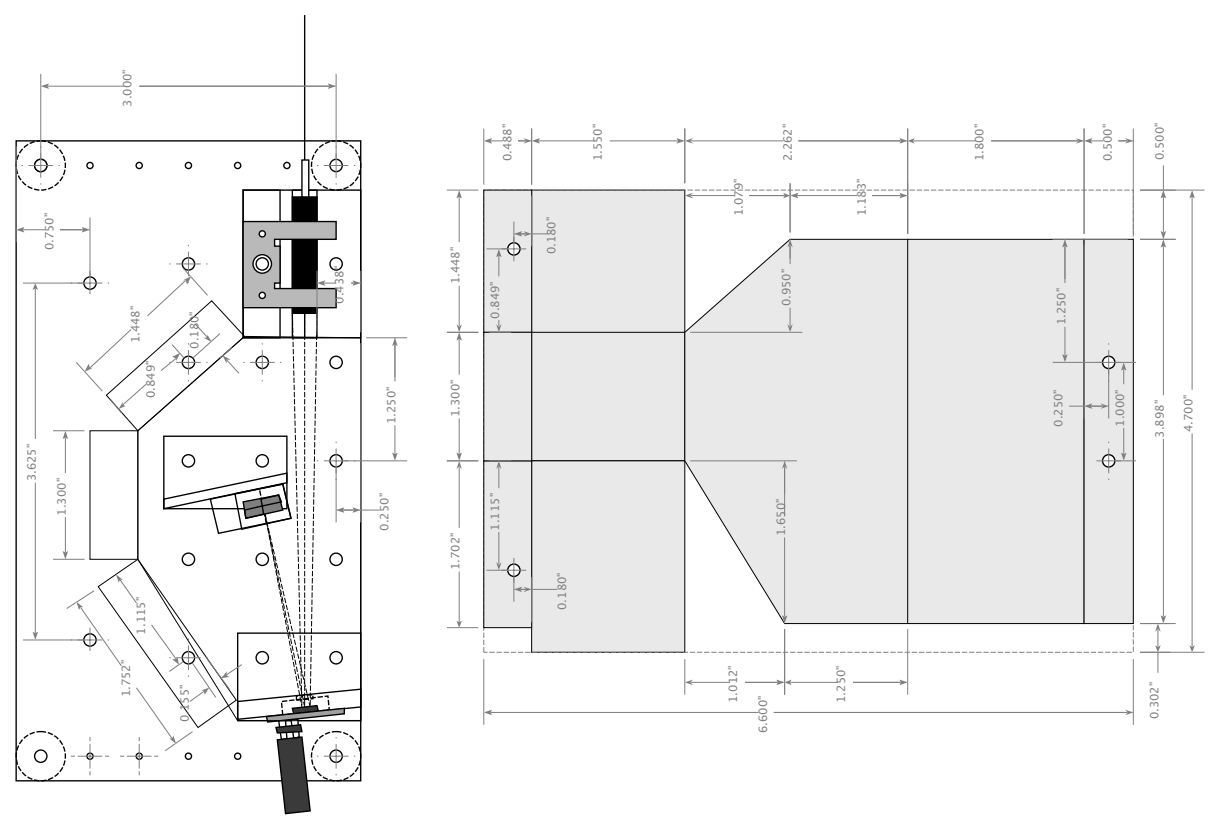

Figure G.5: Design of copper enclosure. 


\section{Holder for Crystal Sample}

Robert Moffatt, Stanford University

March 29, 2013

Part \#1:

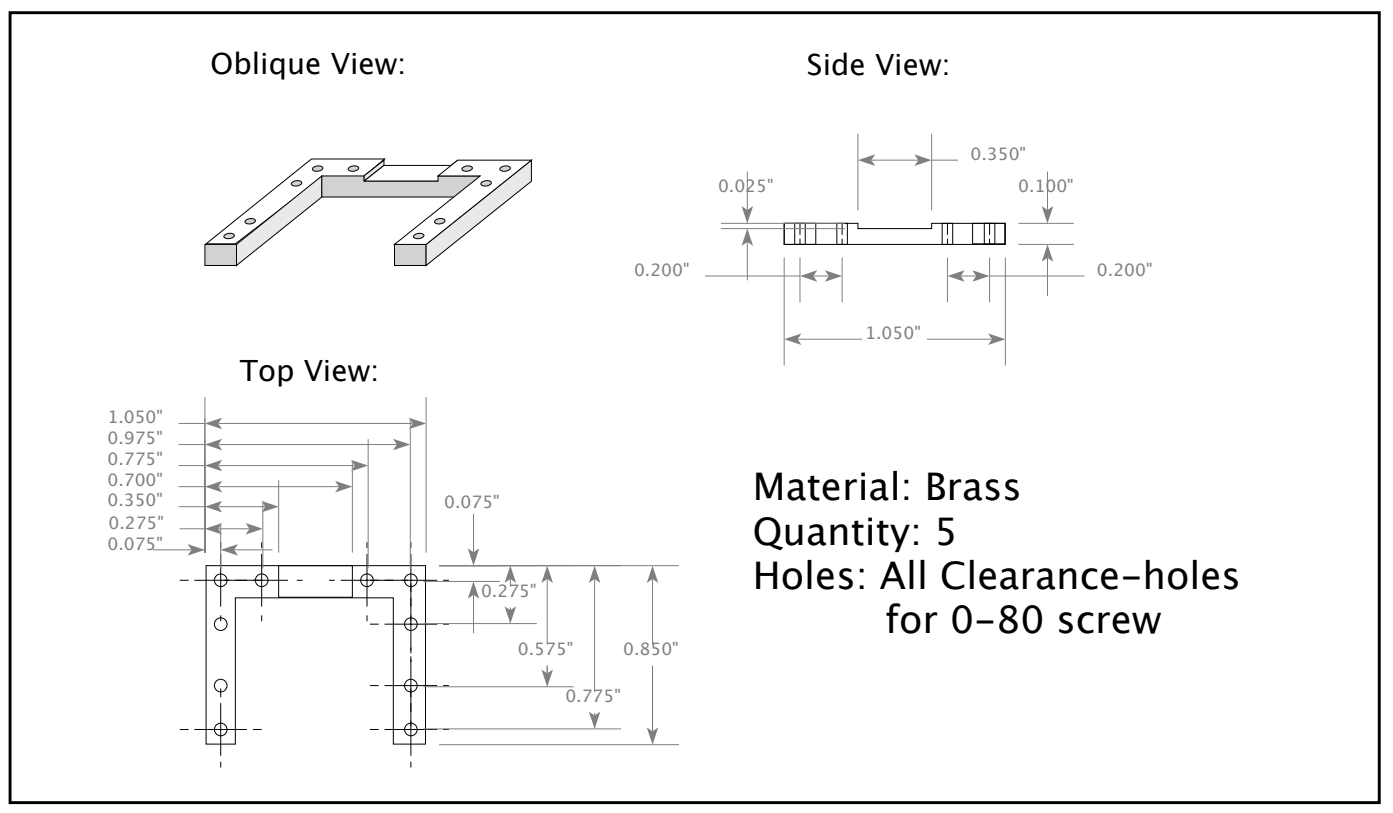

Part \#2: (Same as above, except all holes tapped)

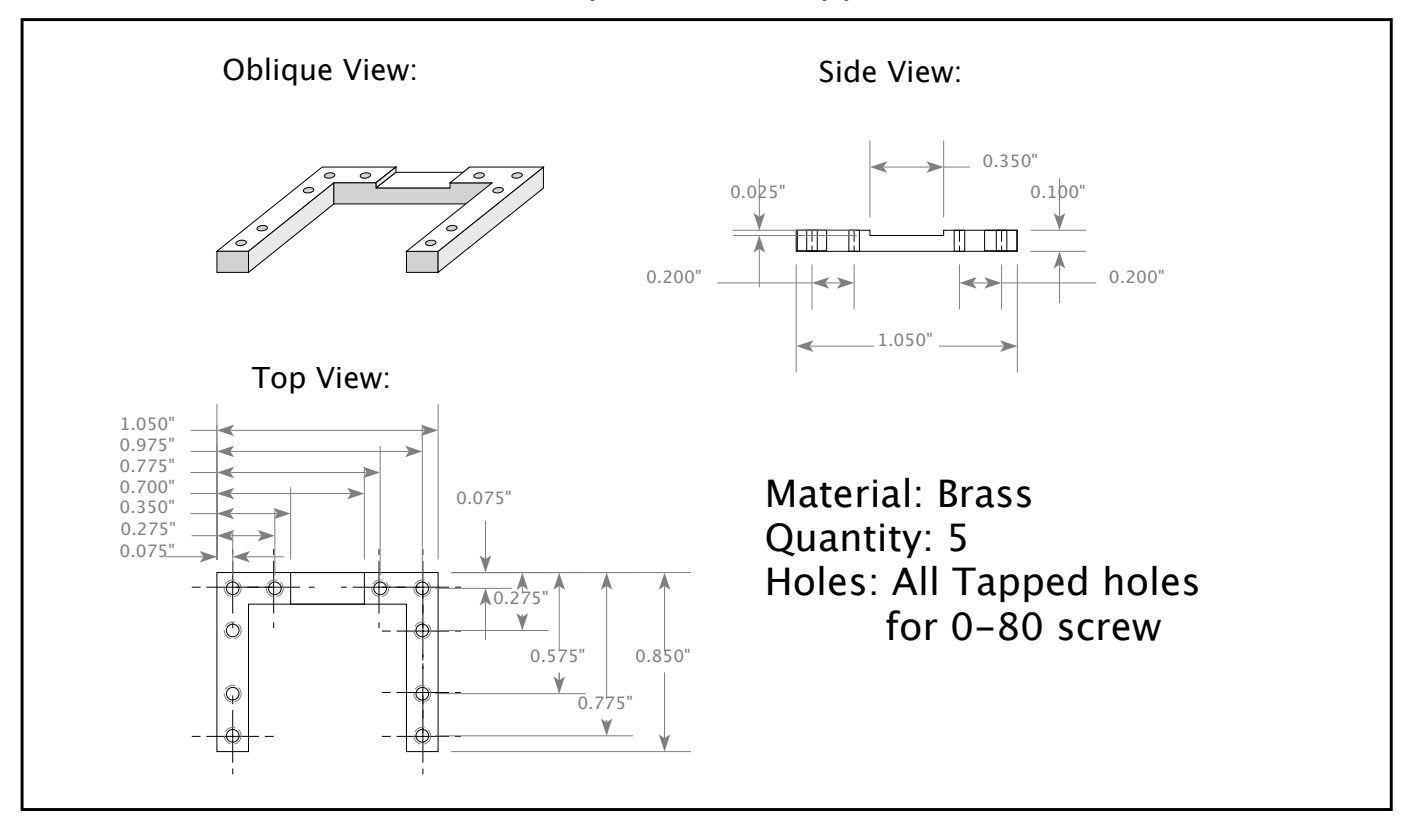

Figure G.6: Crystal sample holder. 


\section{Holder for Crystal Sample}

Robert Moffatt, Stanford University

March 29, 2013

Part \#3:

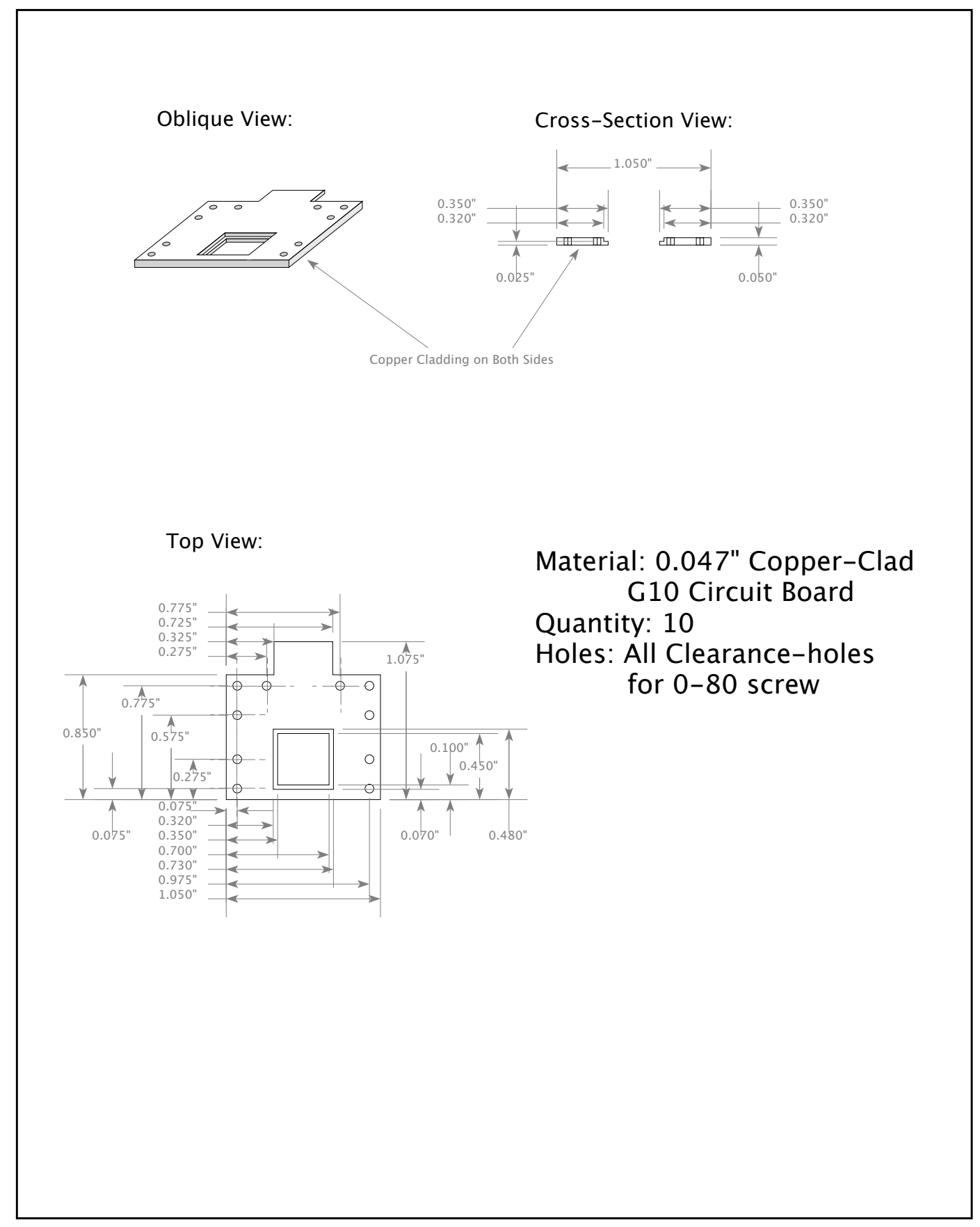

Figure G.7: Crystal sample holder. 


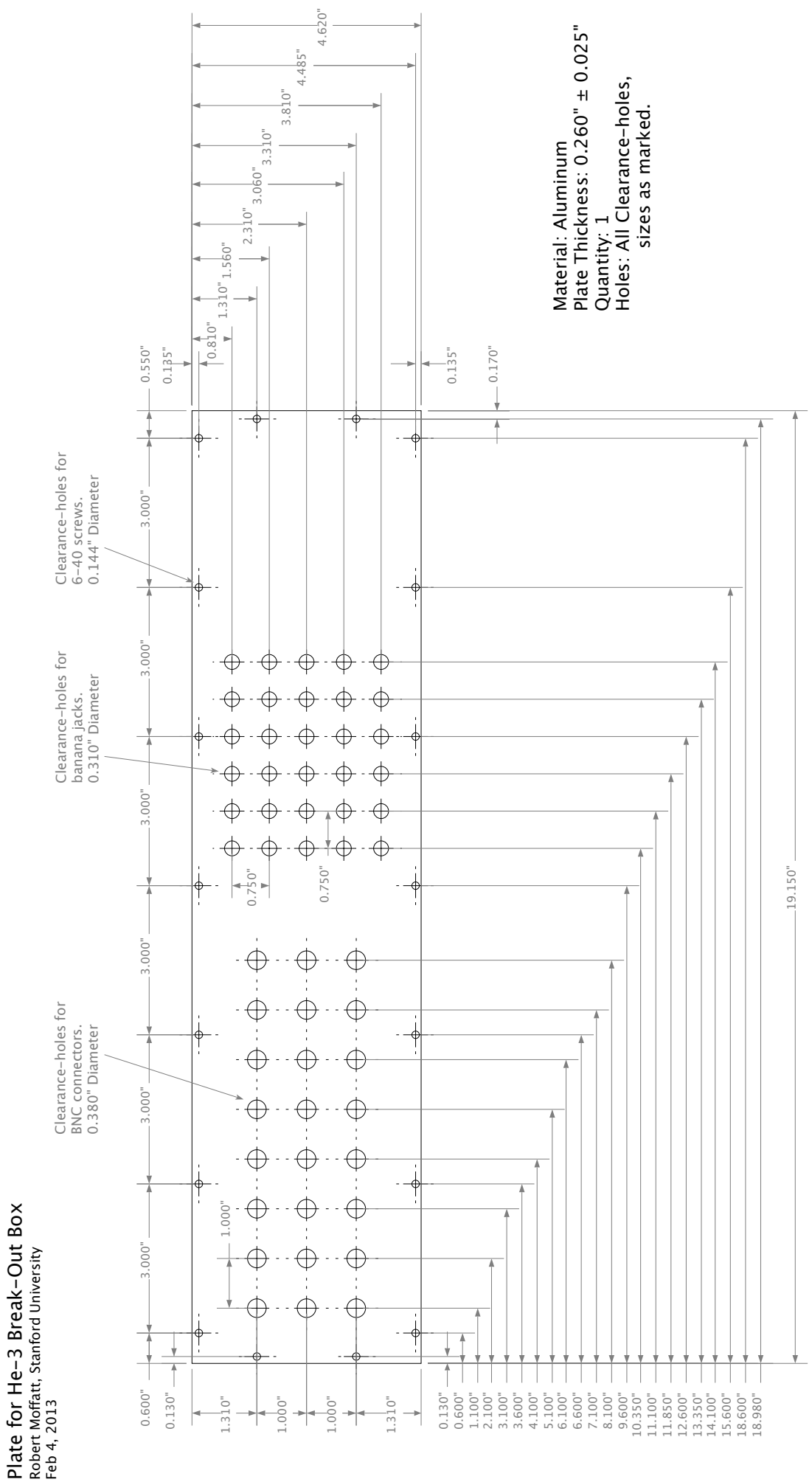

Figure G.8: Break-out box for the ${ }^{3} \mathrm{He}$ cryostat. 


\section{Copper Back-Plate for Cold Amplifier}

Robert Moffatt, Stanford University

March 12, 2014

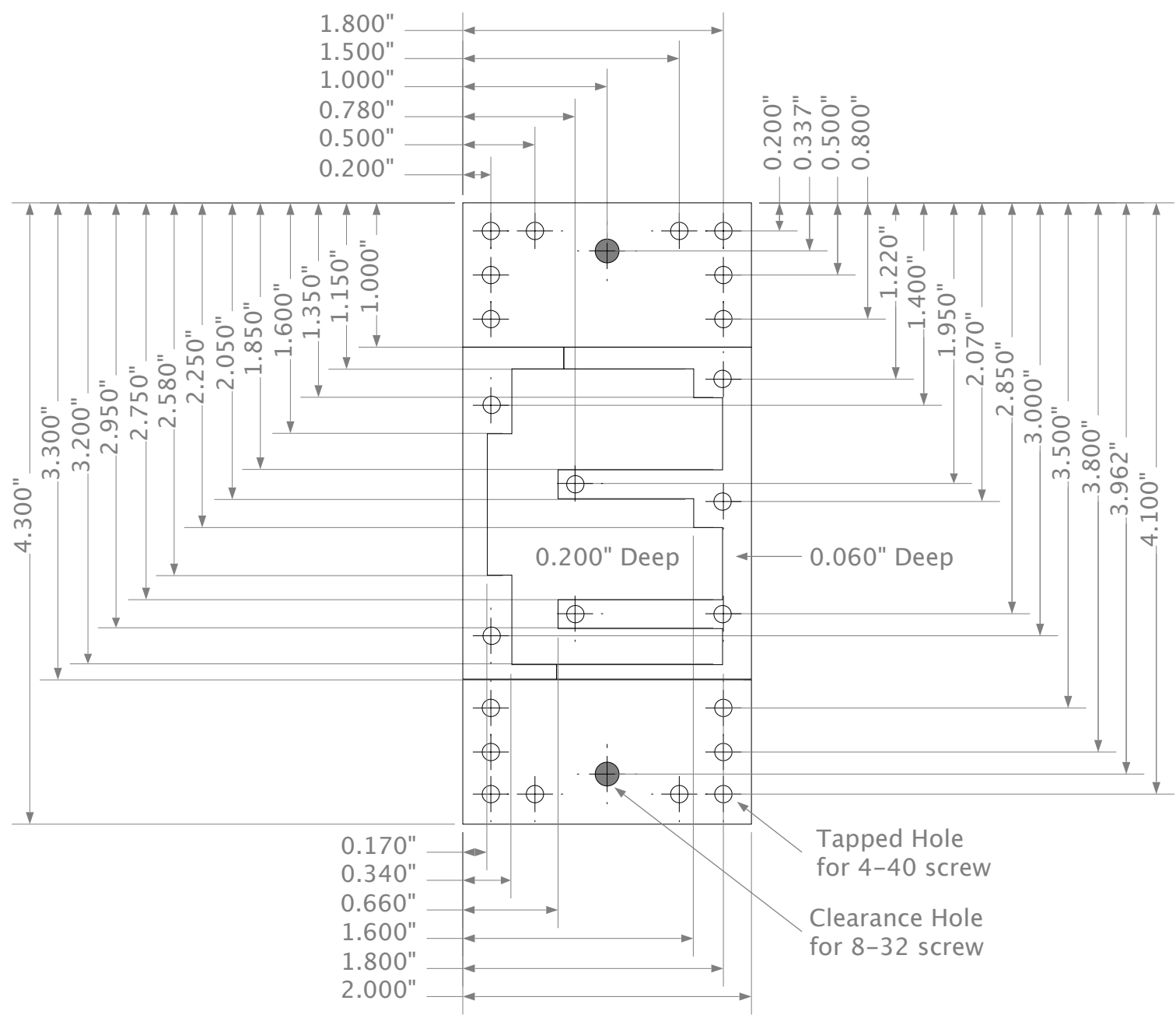

Material: Copper

Quantity: 1

Holes: White $=$ Tapped $4-40$

Gray $=$ Clearance $8-32$

Figure G.9: Copper back plate for the cold amplifier. 
G10 Circuit Board for Cryogenic Amplifier

Robert Moffatt, Stanford University

March 24, 2014

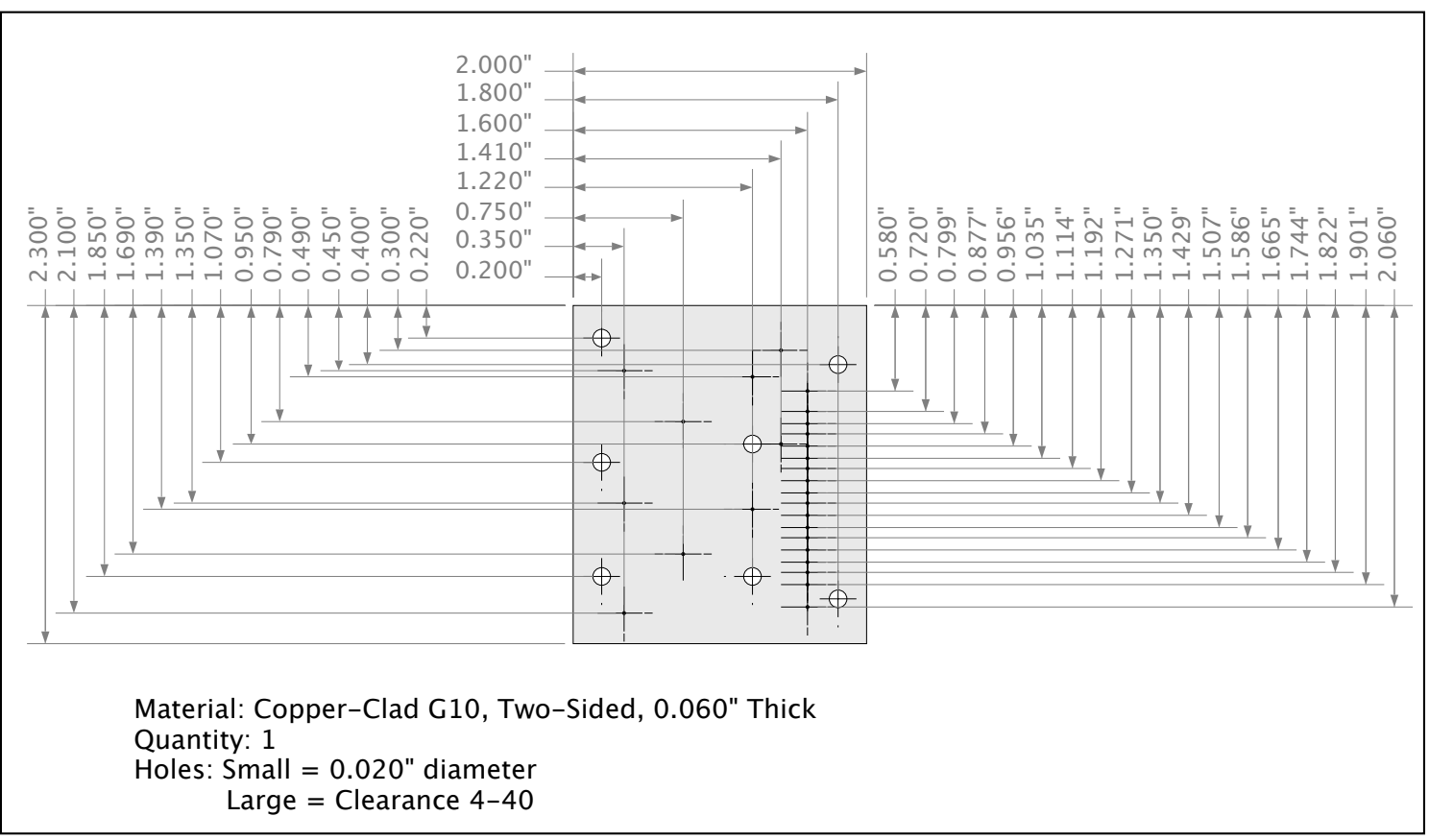

Figure G.10: Circuit board for cold amplifier. 
APPENDIX G. EXPERIMENTAL SETUP - SUPPLEMENTAL MATERIAL 152

Circuit Board Layout for Cryogenic Amplifier Robert Moffatt, Stanford University June 30,2014

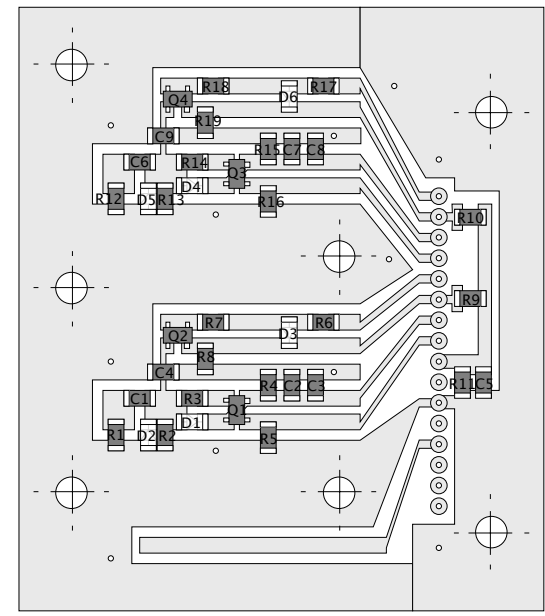

$\mathrm{R} 1, \mathrm{R} 2, \mathrm{R} 12, \mathrm{R} 13=10 \mathrm{M} \Omega(21.5 \mathrm{M} \Omega)$

$\mathrm{R} 3, \mathrm{R} 6, \mathrm{R} 7, \mathrm{R} 9, \mathrm{R} 10, \mathrm{R} 14, \mathrm{R} 17, \mathrm{R} 18=4.99 \mathrm{k} \Omega(7.7 \mathrm{k} \Omega)$

$\mathrm{R} 4, \mathrm{R} 5, \mathrm{R} 11, \mathrm{R} 15, \mathrm{R} 16=680 \mathrm{k} \Omega(1.8 \mathrm{M} \Omega)$

$\mathrm{R} 8, \mathrm{R} 19=220 \Omega(307 \Omega)$

$\mathrm{C} 1, \mathrm{C} 3, \mathrm{C} 6, \mathrm{C} 8=8.2 \mu \mathrm{F}(420 \mathrm{nF})$

$\mathrm{C} 2, \mathrm{C} 4, \mathrm{C} 5, \mathrm{C} 7, \mathrm{C} 9=100 \mathrm{nF}(8 \mathrm{nF})$

D1, D2, D3, D4, D5, D6 = RLS4 150TE-11

Q1, Q2, Q3, Q4 = 3SK165

All values not in parenthesis are nominal. Parenthesis indicate values at $4 \mathrm{~K}$.

Figure G.11: Component layout for cold amplifier. 


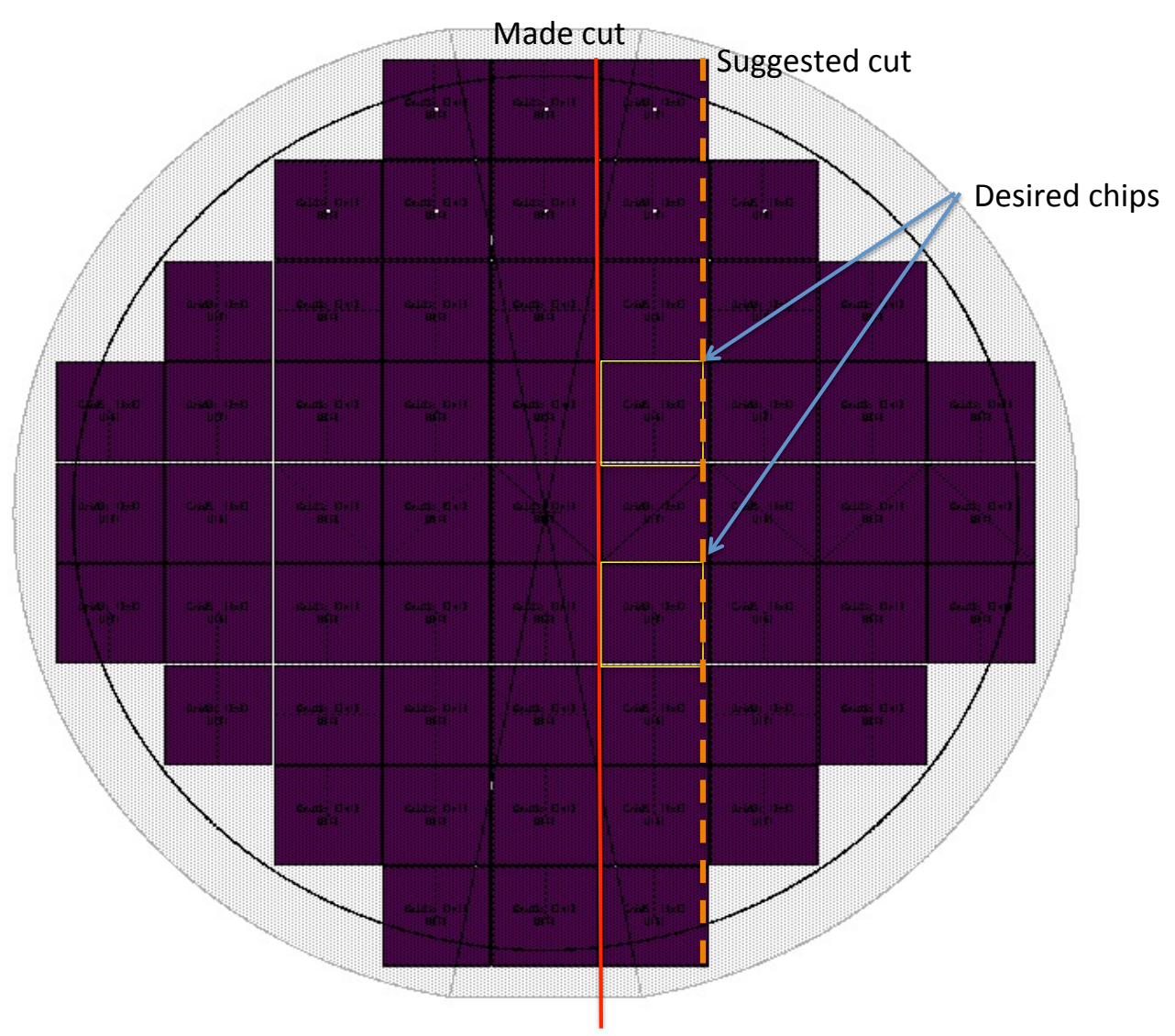

Figure G.12: Germanium crystal wafer used as source for crystal test samples. 


\section{G.2 Amplifier Circuit}

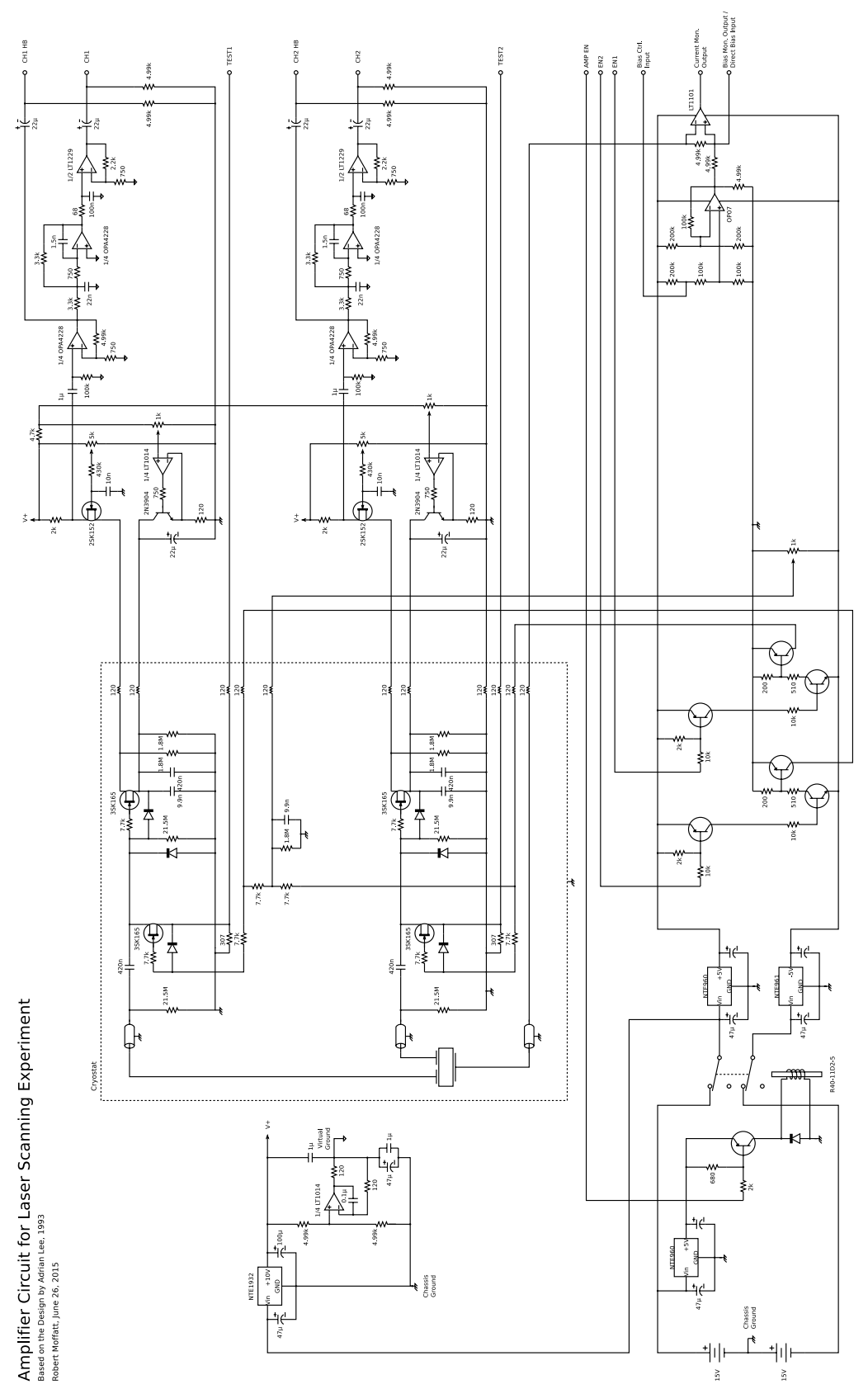

Figure G.13: Full schematic for the amplifier circuit. The dotted line indicates the part of the circuit contained within the cryostat. The rest of the circuit is at room temperature. 


\section{G.3 Photos}

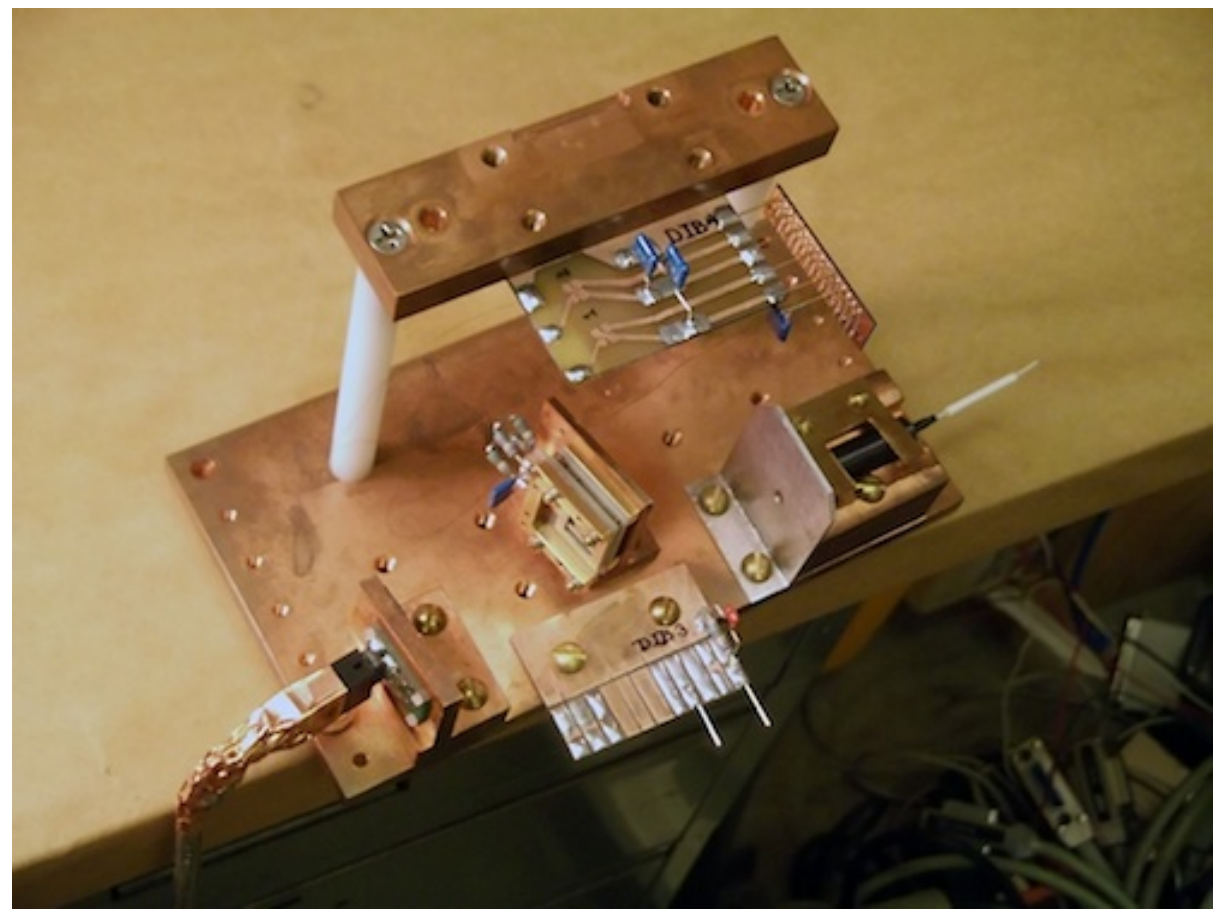

Figure G.14: Version 1 of the cold amplifier. This version used single wires to connect the board to the crystal bias electrode and the charge collection electrodes. It oscillated badly. 


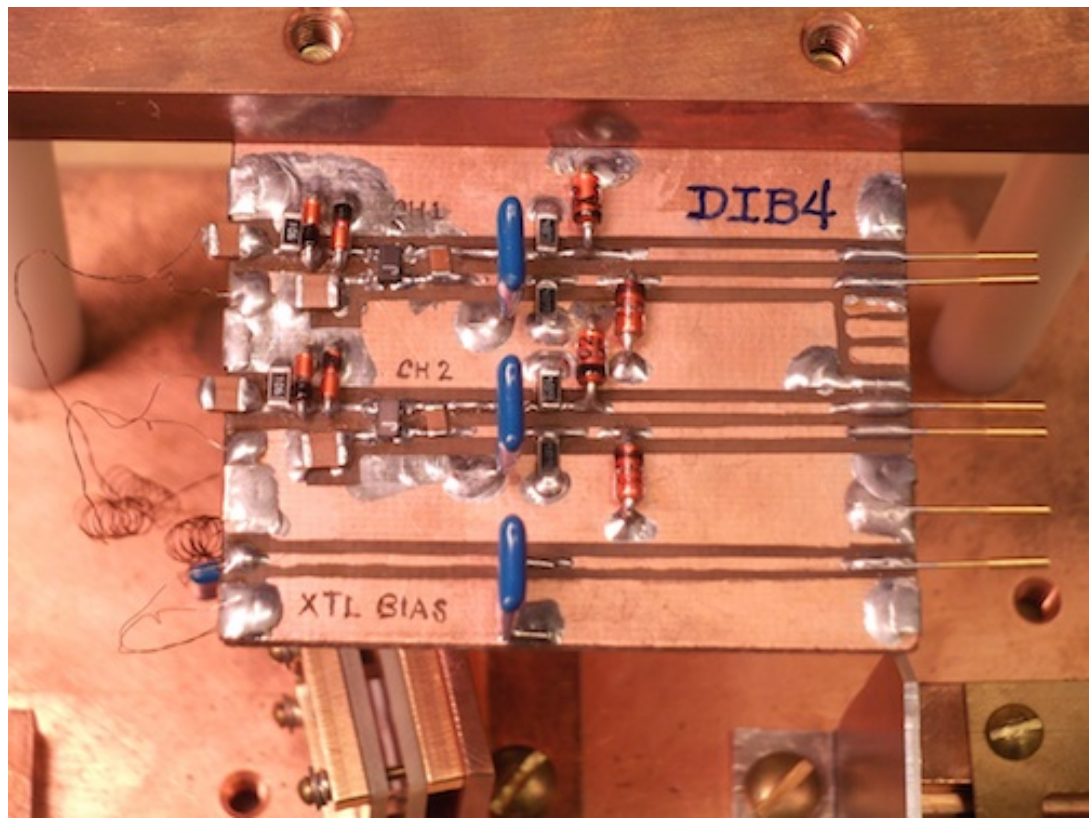

(a) Twisted pair connections.

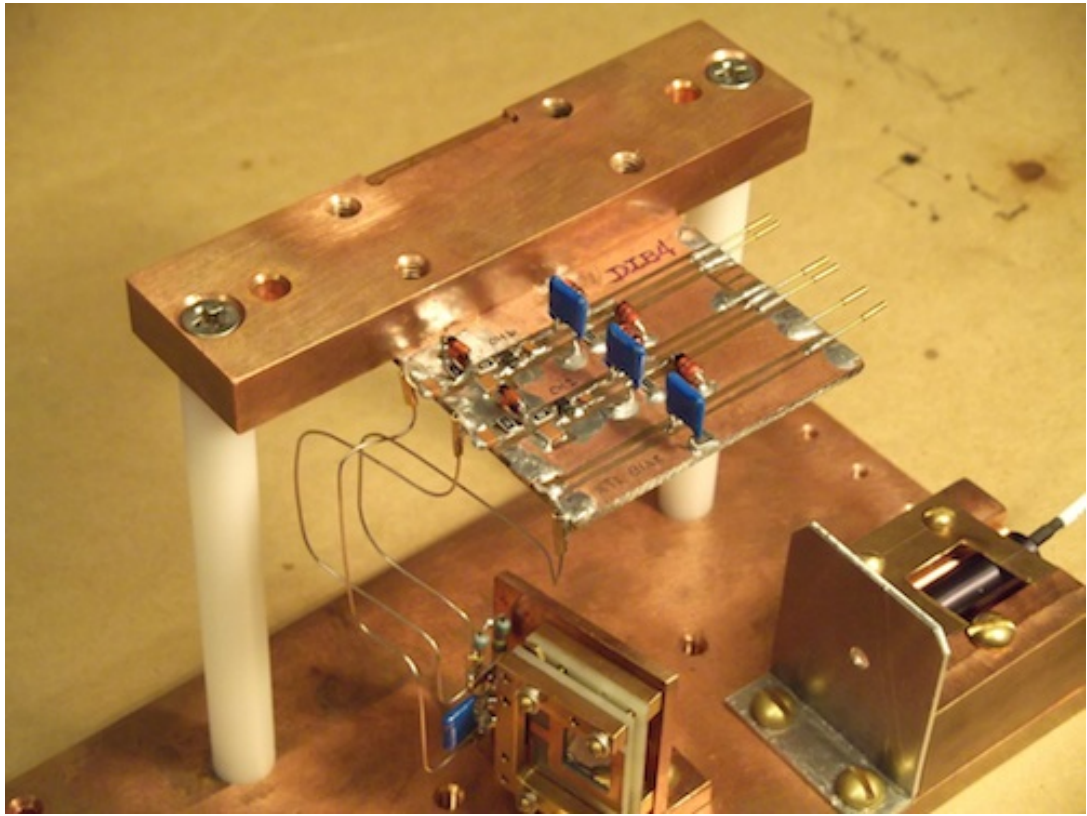

(b) Coax connections.

Figure G.15: Version 2 of the cold amplifier. Twisted pairs were introduced (top) in order to reduce capacitive coupling between the input and output in an attempt to avoid oscillation. However, oscillation was still a problem, so coaxial lines were installed instead (bottom). The coax helped avoid oscillation, but the circuit still oscillated under certain bias conditions. 


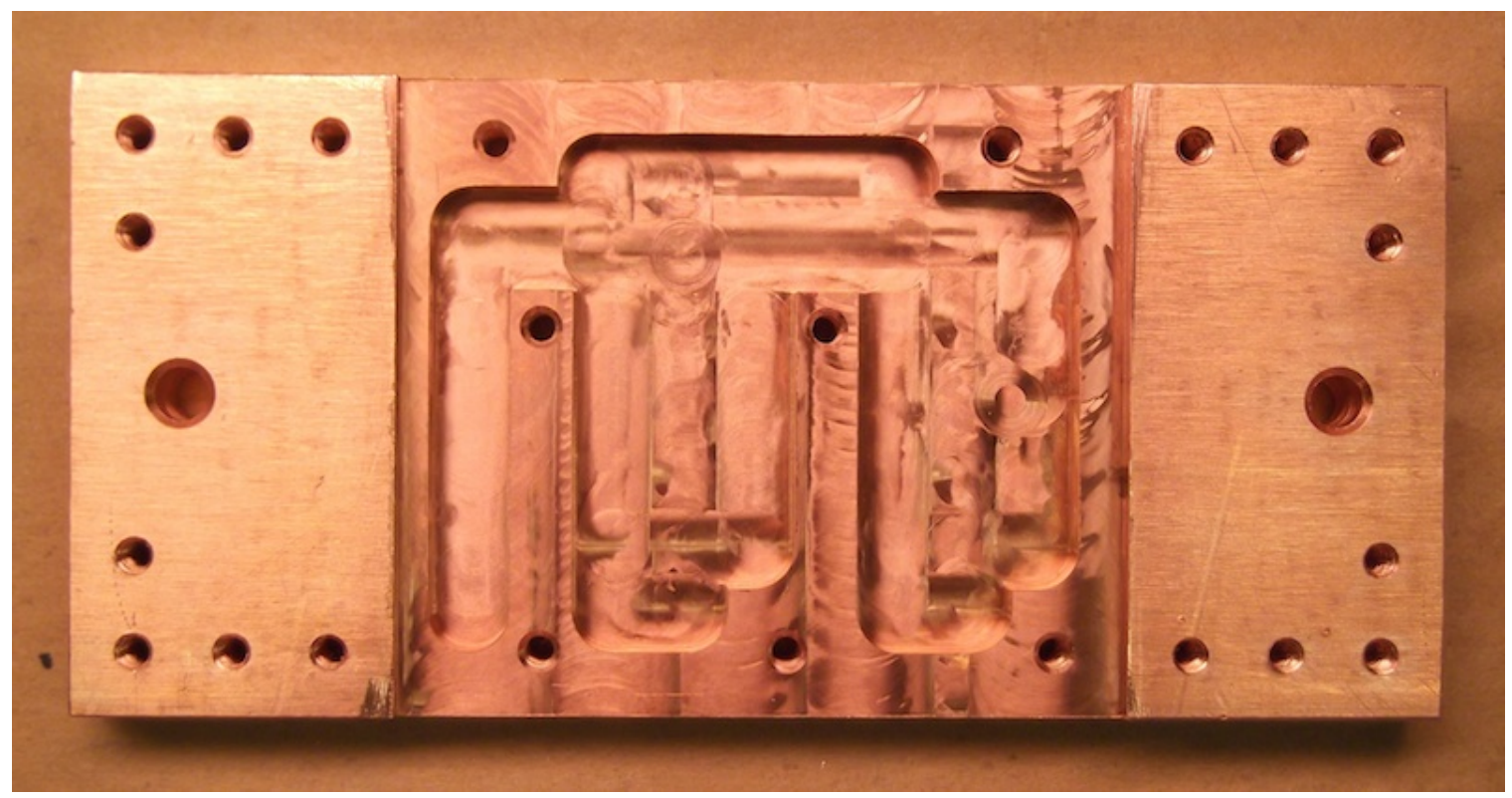

(a) Copper backplate for cold amp.

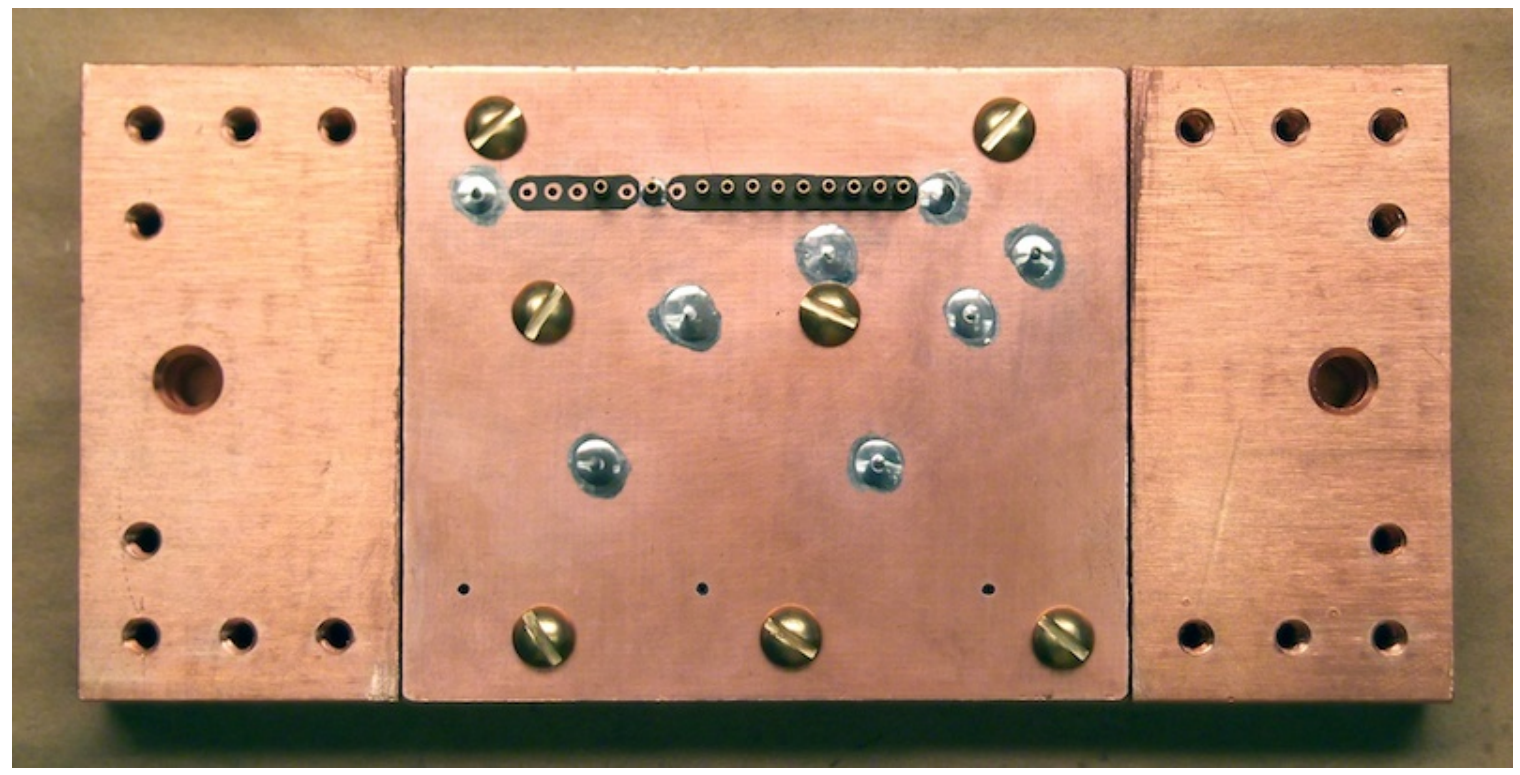

(b) Backplate with cold amp board installed.

Figure G.16: Copper plate for version 3 of the cold amplifier. This backplate was intended to fully shield the circuit to prevent capacitive or inductive coupling from producing any unintended positive feedback loops. 

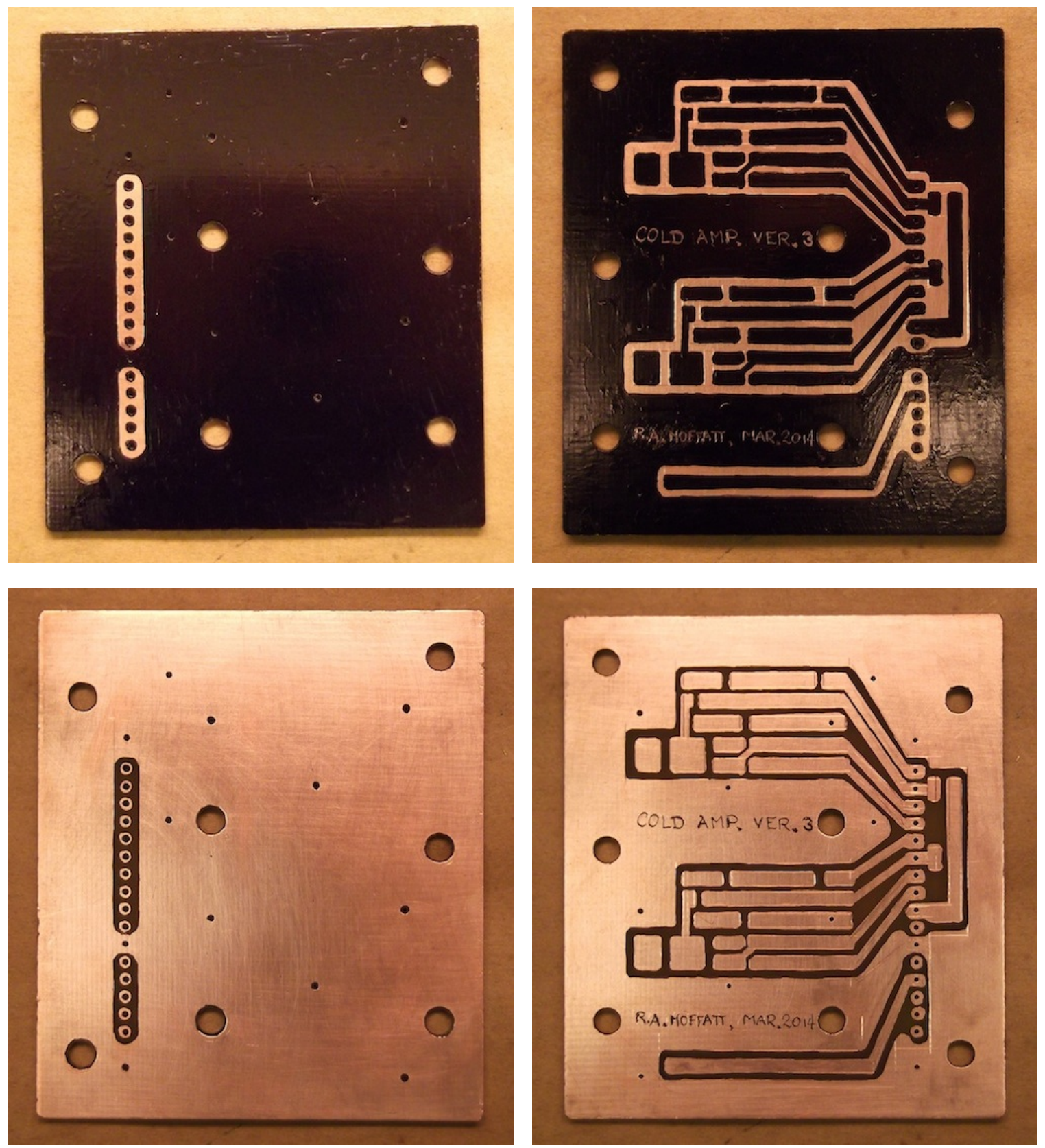

Figure G.17: Circuit board for version 3 of the cold amplifier before etching (top) and after etching (bottom). 


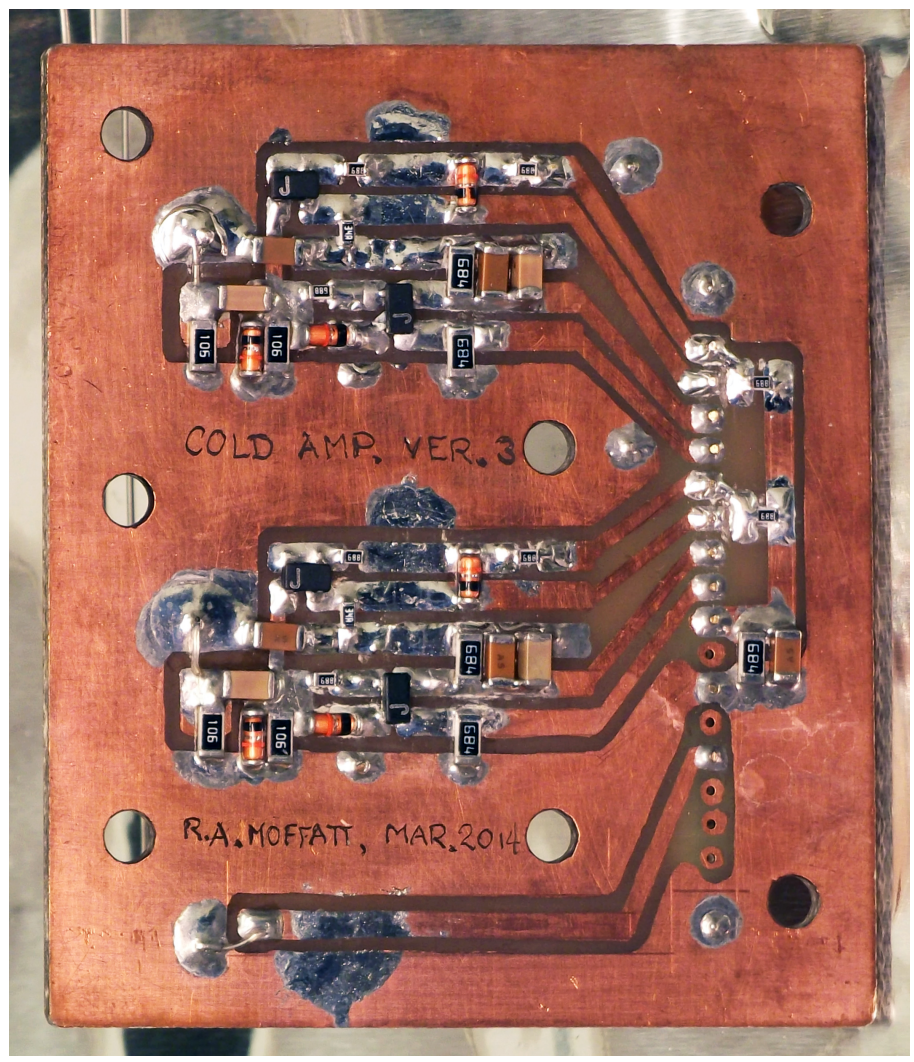

(a) Cold amplifier board.

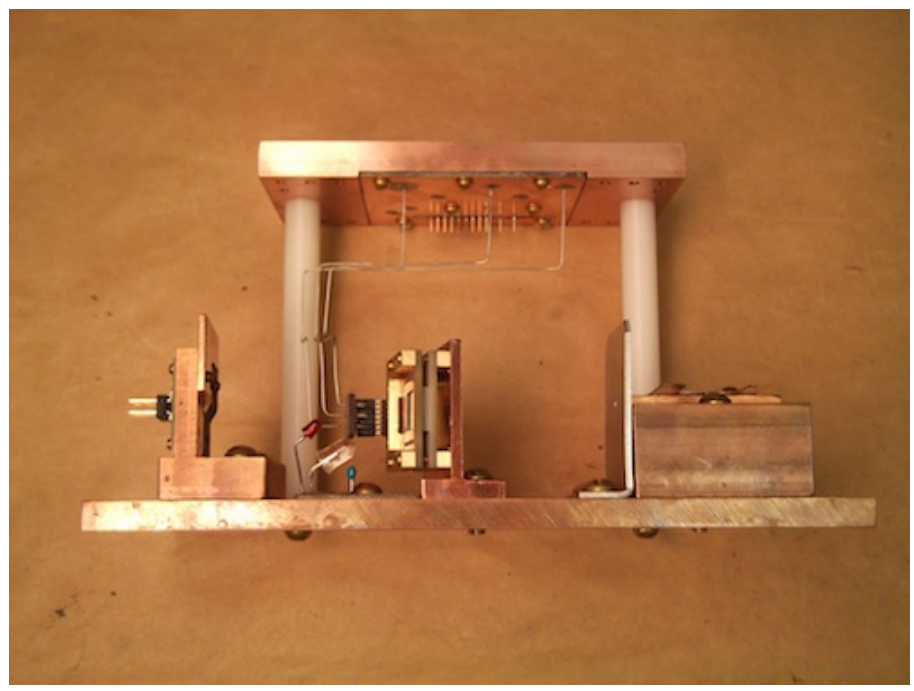

(b) Cold amplifier mounted on payload.

Figure G.18: Version 3 of the cold amplifier. The inputs in this version were completely shielded. Oscillation was not a problem in this version. 

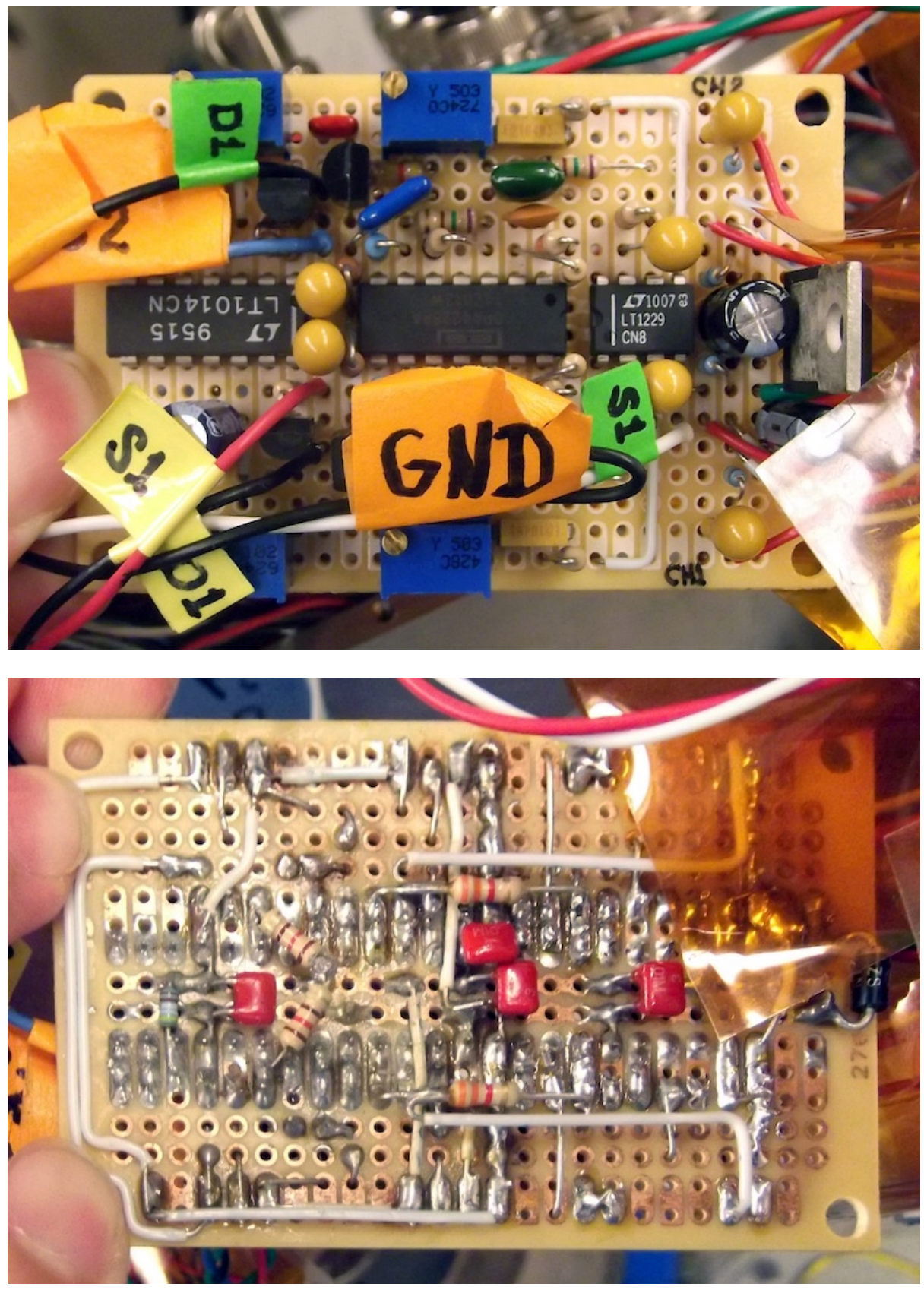

Figure G.19: Circuit board for the warm amplifier. 

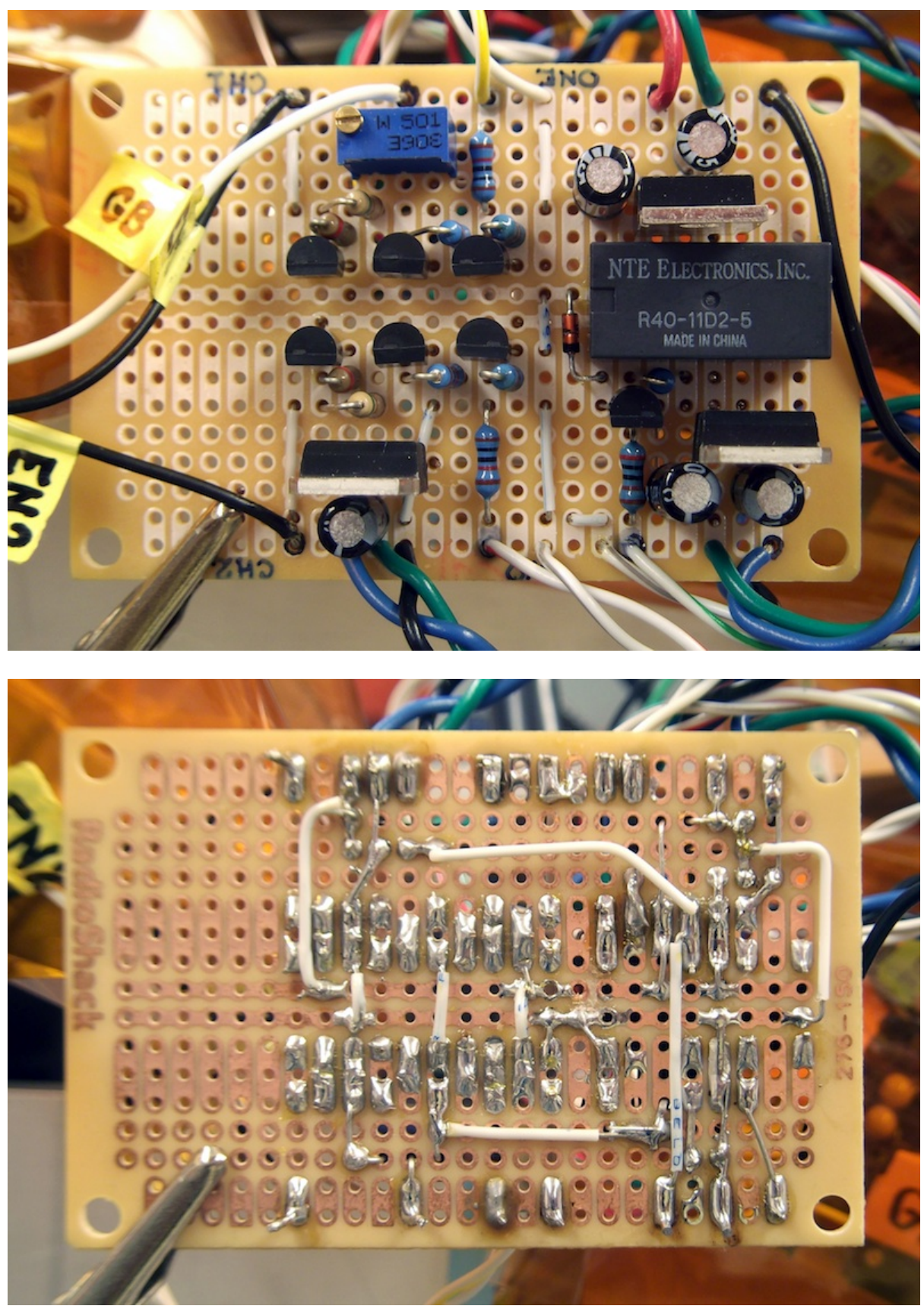

Figure G.20: Circuit board for the control circuit. 

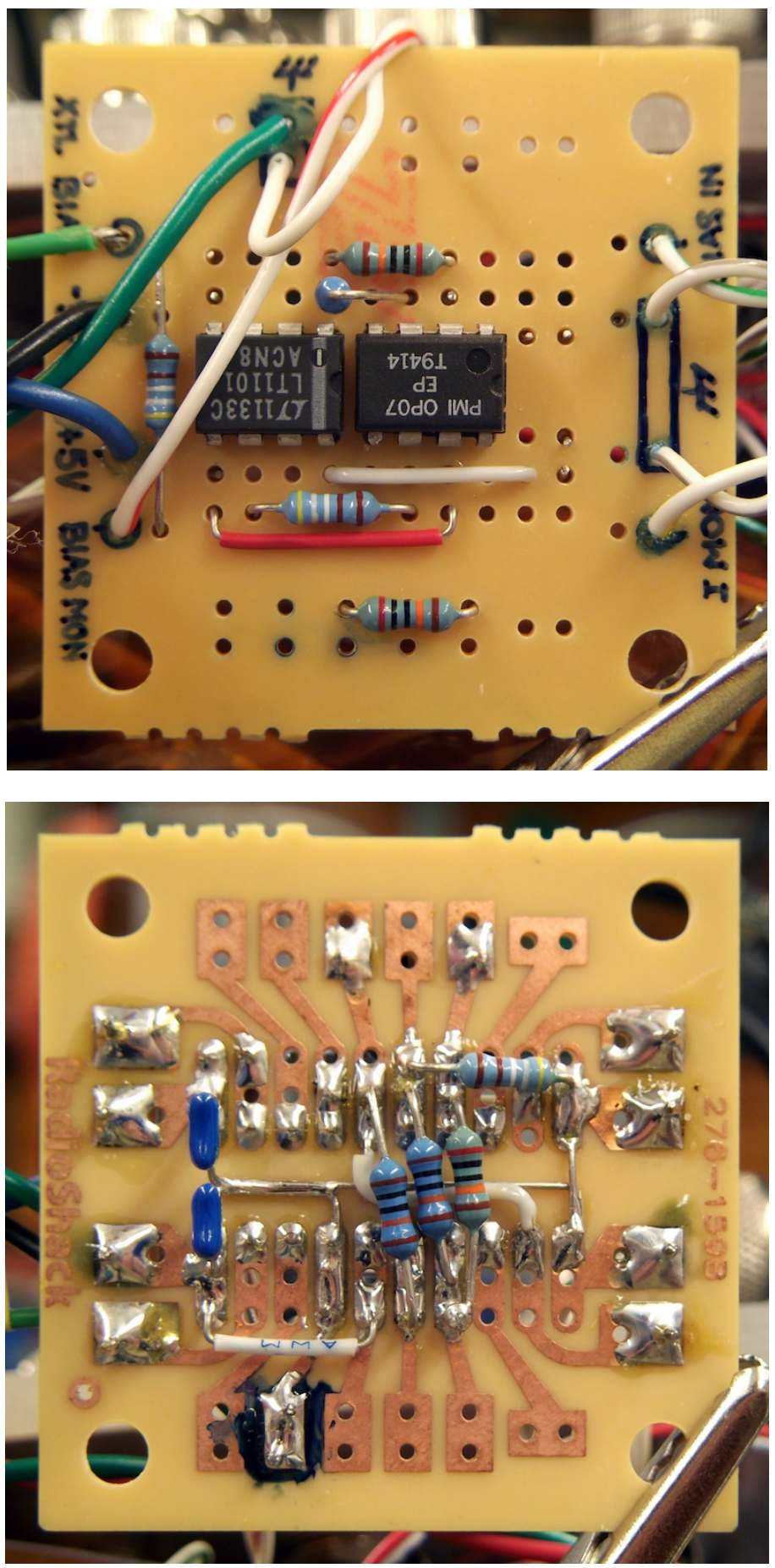

Figure G.21: Circuit board for the bias circuit. 


\section{G.4 Transfer Function}

Least-squares fits were performed for the channel 1 and channel 2 transfer functions using rational function models of the form:

$$
H(s)=K \frac{\prod_{i}\left(s-z_{i}\right)}{\prod_{j}\left(s-p_{j}\right)}
$$

A model using five zeros and eight poles was found to fit well. The best fit parameters are given in Table G.1. The locations of the poles and zeros are plotted in Figure G.22

\begin{tabular}{|l|l|l|}
\hline \multicolumn{1}{|c|}{$H_{1}:$} & \multicolumn{1}{c|}{$H_{2}:$} \\
\hline$K=3.213 \mathrm{E} 13$ \\
$z_{1}=0.041085$ \\
$z_{2}=-0.066912$ \\
$z_{3}=-28013$ \\
$z_{4}=-3199.7-12273 i$ \\
$z_{5}=-3199.7+12273 i$ \\
$p_{1}=-251.83$ \\
$p_{2}=-17.465$ \\
$p_{3}=-113730+3.1271 i$ \\
$p_{4}=-3004.8-12789 i$ \\
$p_{5}=-49161-100320 i$ \\
$p_{6}=-113730-3.1271 i$ \\
$p_{7}=-3004.8+12789 i$ \\
$p_{8}=-49161+100320 i$
\end{tabular} \mid \begin{tabular}{ll}
$K=$ \\
$z_{1}=1.2094$ \\
$z_{2}=-0.24422$ \\
$z_{3}=-33714$ \\
$z_{4}=-3060.3-12273 i$ \\
$z_{5}=-3060.3+12273 i$ \\
$p_{1}=-17.331$ \\
$p_{2}=-190.43$ \\
$p_{3}=-100880+3.1271 i$ \\
$p_{4}=-2820.7-12789 i$ \\
$p_{5}=-48005-100320 i$ \\
$p_{6}=-100880-3.1271 i$ \\
$p_{7}=-2820.7+12789 i$ \\
$p_{8}=-48005+100320 i$ \\
\hline
\end{tabular}

Table G.1: Poles and zeros for $H_{1}$ and $H_{2}$ in units of $\mathrm{Hz}$. 


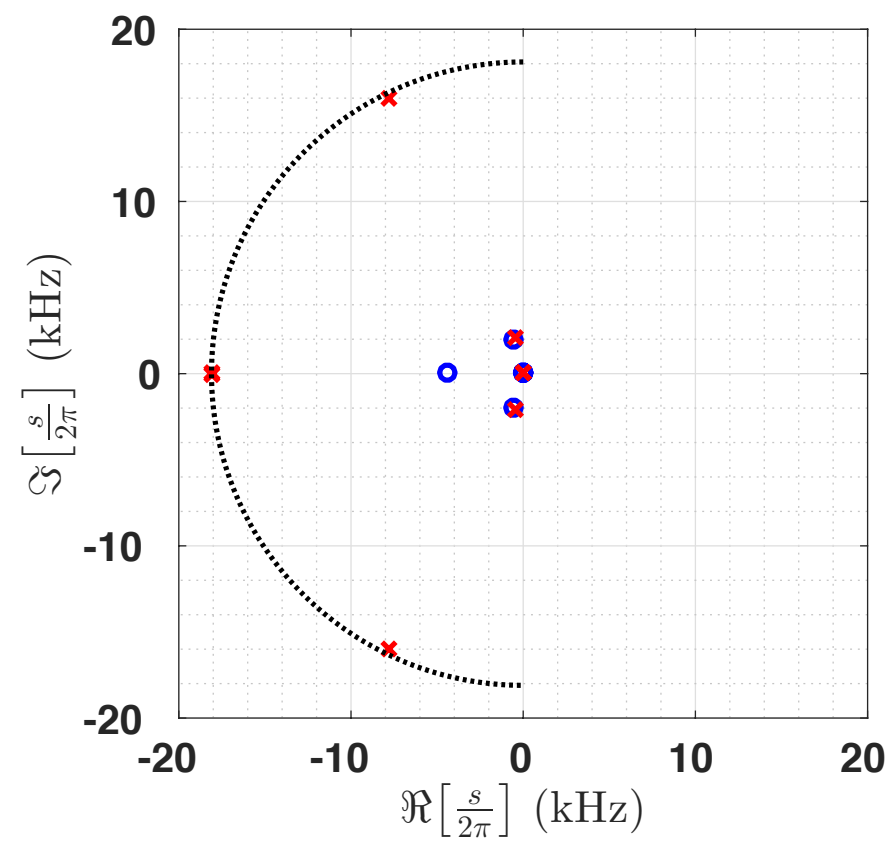

(a) Ch1 transfer function.

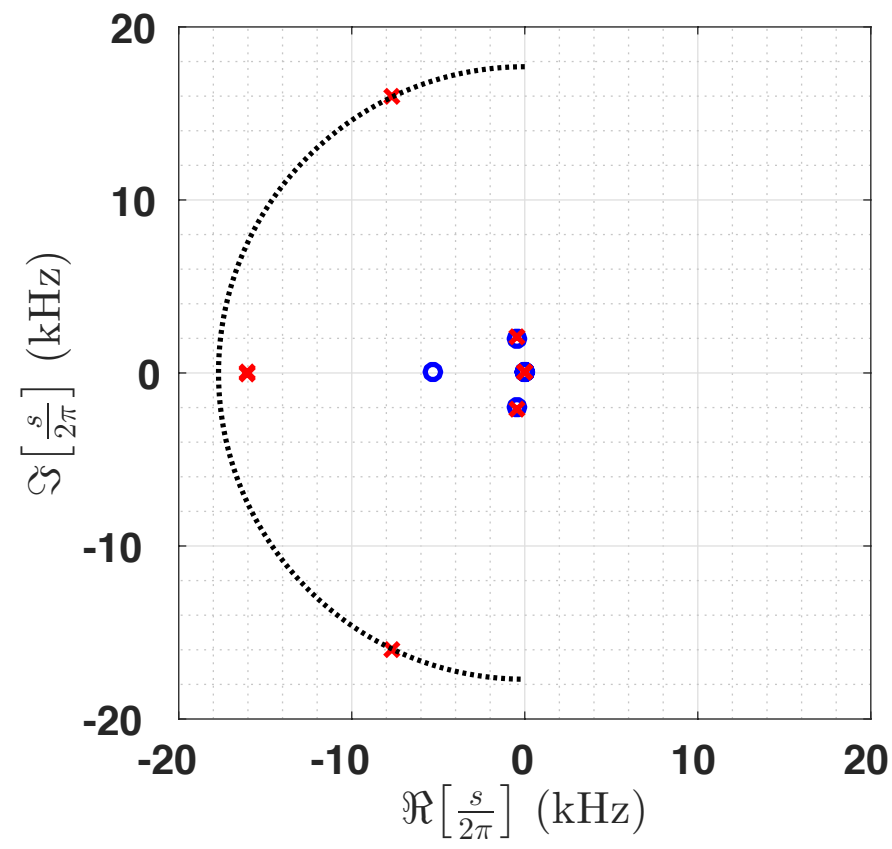

(b) Ch2 transfer function.

Figure G.22: Poles and zeros for the channel 1 and channel 2 transfer functions. Each pole is denoted by a red "x", and each zero is denoted by a blue "o". 


\section{G.5 Noise}

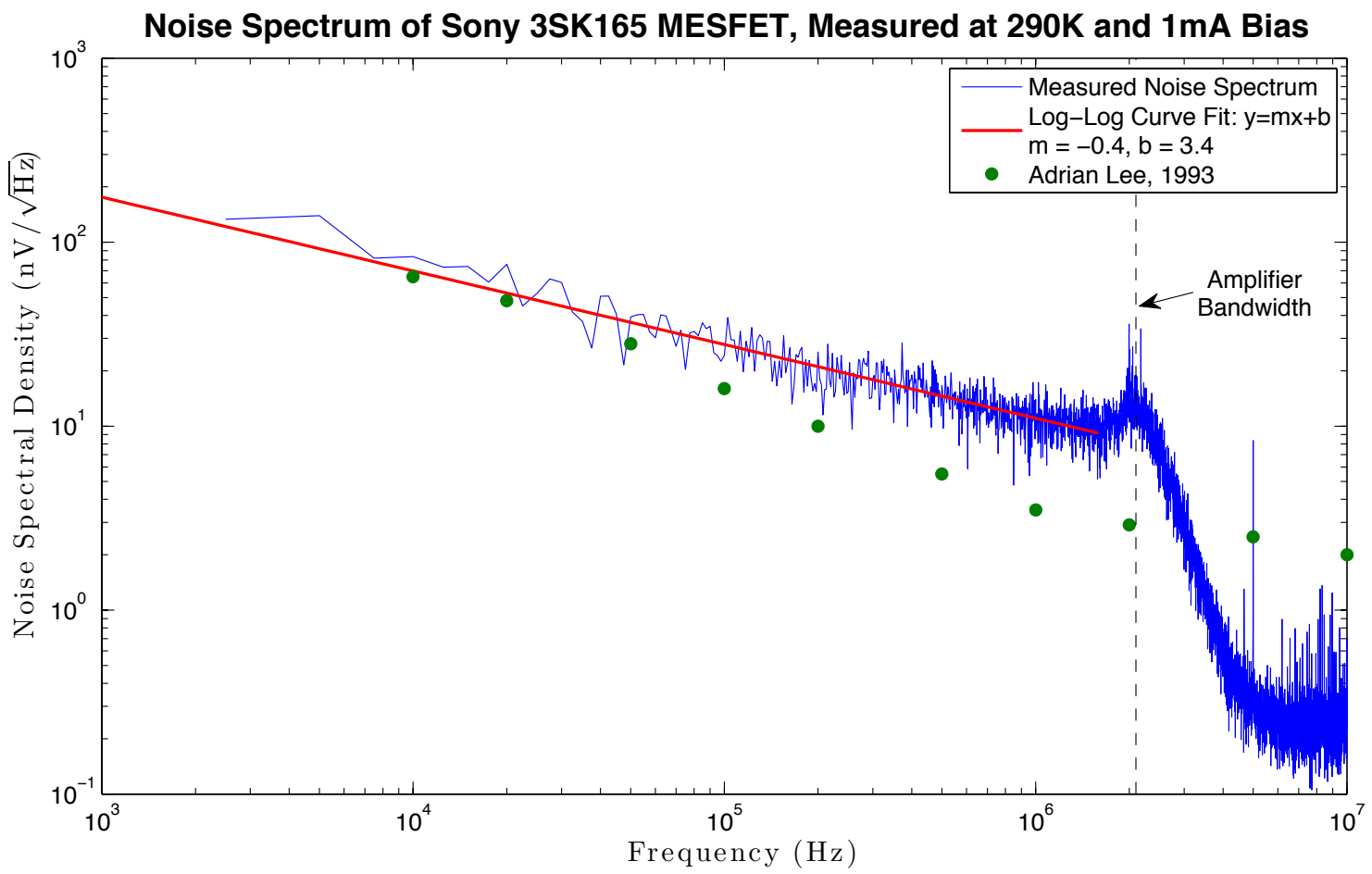

Figure G.23: Noise spectrum of Sony 3SK165 MESFET, measured at 290K and 1mA bias. Measurements from Lee in [60] for the same model transistor at 293K are plotted for comparison. 


\section{G.6 ${ }^{3}$ He Cryostat}

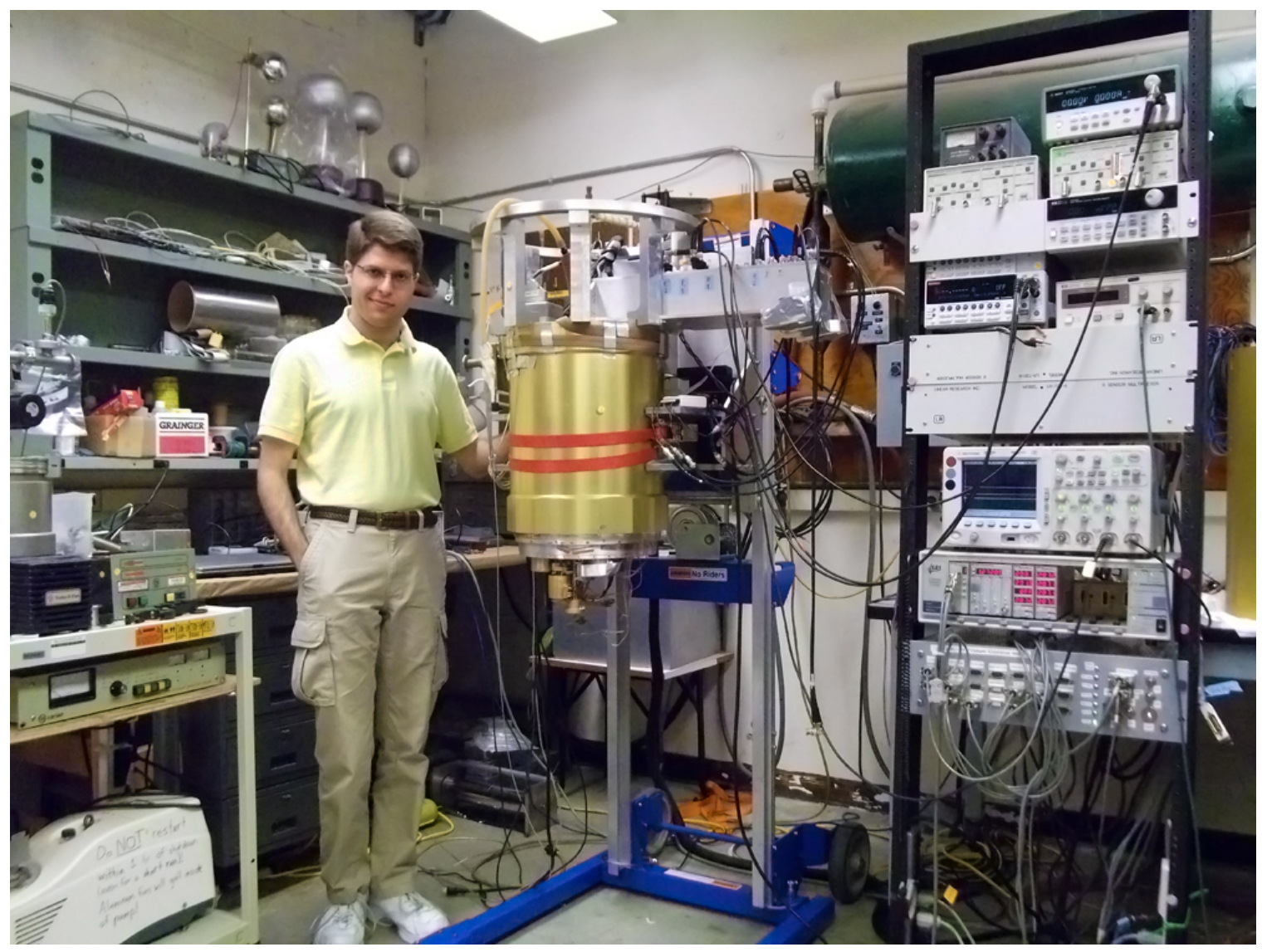

Figure G.24: Photograph of the ${ }^{3} \mathrm{He}$ cryostat and laboratory equipment used in the charge transport experiment. 


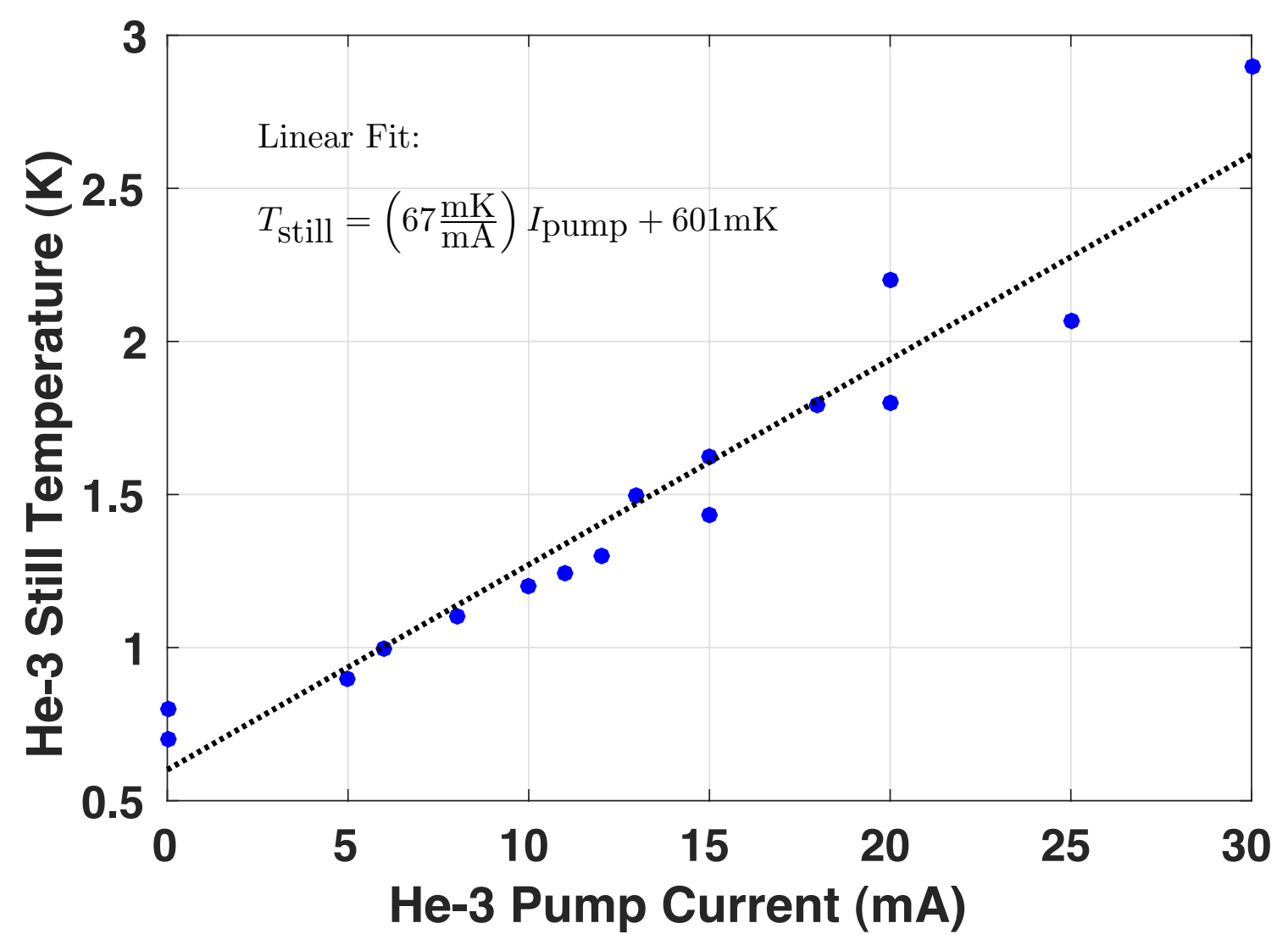

Figure G.25: Measurements taken by James Allen of the ${ }^{3} \mathrm{He}$ still temperature as a function of the electrical current applied to the resistive heater in the ${ }^{3} \mathrm{He}$ pump with the heat switch turned off. Raising the temperature of the pump increases the equilibrium vapor pressure of ${ }^{3} \mathrm{He}$ in the ${ }^{3} \mathrm{He}$ still, which raises the temperature of the boiling point. This effect was investigated as a method for actively controlling the temperature of the payload without adding heat to the ${ }^{3} \mathrm{He}$ still, which would reduce the hold time at base temperature. The power dissipated by the heater can be found

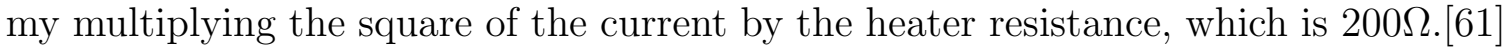
This power is dissipated into the $4 \mathrm{~K}$ helium bath, and causes an increase in the rate of helium boil-off. 


\section{G.7 Control System}

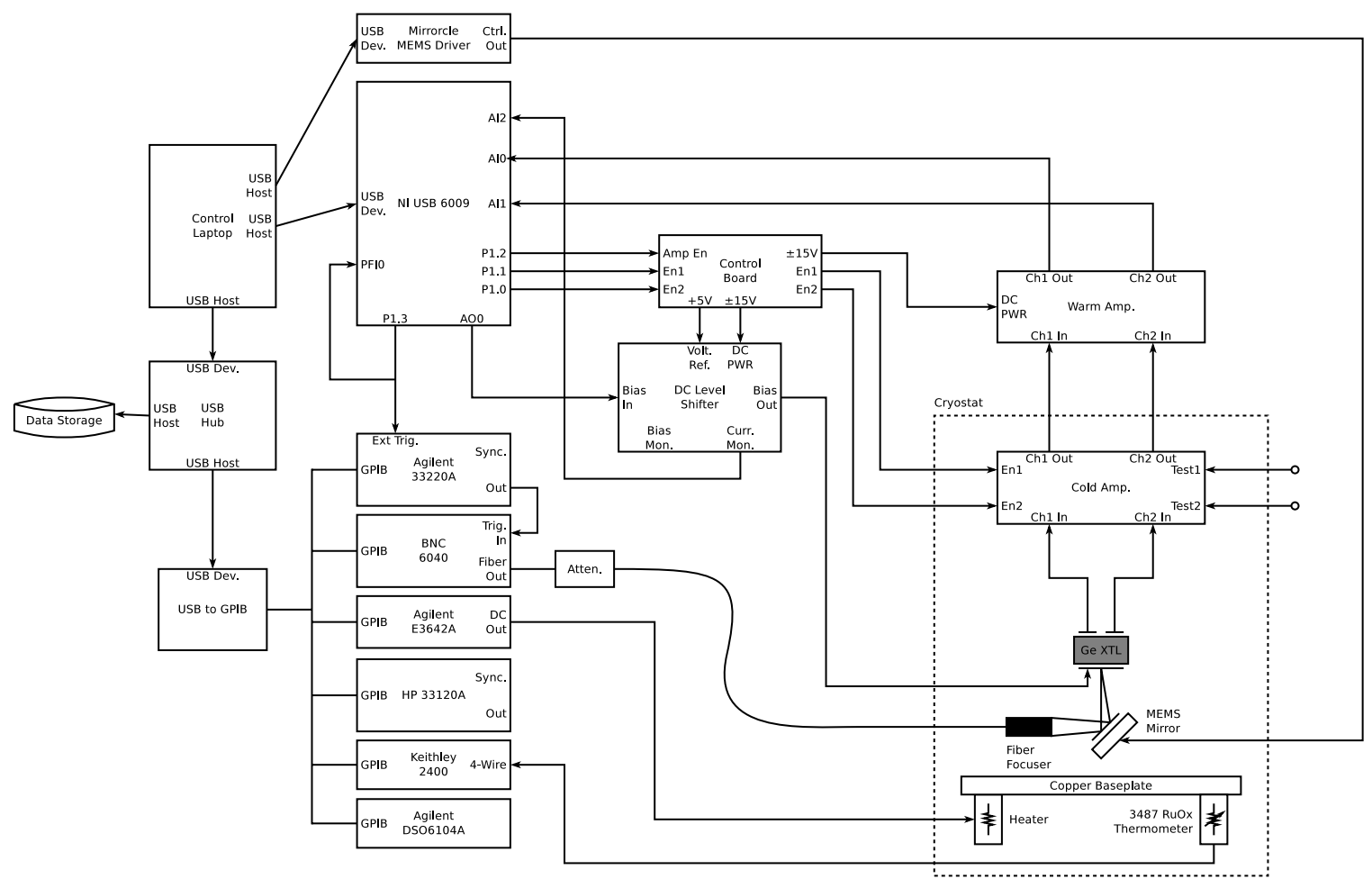

Figure G.26: Detailed block diagram for the experiment control system. 


\section{Appendix $\mathbf{H}$}

\section{Maximum Likelihood Linear Fits}

In this section, a formula is derived for the maximum-likelihood fit of a model to a measurement, assuming the set of all possible models forms a linear vector space, and the measurement can be accurately represented as a pure (noiseless) signal plus Gaussian noise.

\section{H.1 Signal Vectors and Inner Products}

Assume a Nyquist band-limited continuous signal is sampled $N$ times over a time period $\mathscr{T}$ with a time interval of $\Delta t=\mathscr{T} / N$ between each sample. The signal may be represented by an $N$-dimensional vector whose components are equal to the sample values, $a[t]$ at each sampling time. The signal vector may also be decomposed into Fourier components, $A[\omega]$, defined in the following way:

$$
\begin{gathered}
A[\omega] \equiv \Delta t \sum_{t} a[t] e^{-i \omega t} \\
a[t]=\Delta \nu \sum_{\omega} A[\omega] e^{i \omega t} \\
\Delta \nu \equiv 1 / \mathscr{T} \quad, \quad \Delta \omega \equiv 2 \pi \Delta \nu
\end{gathered}
$$


where the variables $t$ and $\omega$ take on only discrete values:

$$
\begin{aligned}
t & =0, \Delta t, 2 \Delta t, \ldots,(N-1) \Delta t \\
\omega & =\ldots,-\Delta \omega, 0, \Delta \omega, \ldots
\end{aligned}
$$

In the limit where $\Delta t \rightarrow 0$ and $\mathscr{T} \rightarrow \infty$, these equations become:

$$
\begin{aligned}
A(\omega) & =\int d t a(t) e^{-i \omega t} \\
a(t) & =\int d \nu A(\omega) e^{i \omega t}
\end{aligned}
$$

Let the inner product between two signal vectors be defined in the following way:

$$
a \cdot b \equiv \Delta t \sum_{t} a[t] b[t]
$$

If the sample amplitudes, $a[t]$, are scaled such that their squares, $a^{2}[t]$, are equal to the instantaneous power, then the inner product of the vector, $a$, with itself is equal to the total energy contained in the signal:

$$
\mathscr{E}_{s}=a \cdot a=\Delta t \sum_{t} a^{2}[t] \rightarrow \int d t a^{2}(t)
$$

The inner product of two vectors may also be written in terms of the Fourier components:

$$
\begin{aligned}
a \cdot b & =\Delta t \sum_{t} a[t] b[t]=\frac{\mathscr{T}}{N} \sum_{t} \sum_{\omega_{1}, \omega_{2}} \frac{1}{\mathscr{T}^{2}} A\left[\omega_{1}\right] B\left[\omega_{2}\right] e^{i\left(\omega_{1}+\omega_{2}\right) t} \\
& =\frac{1}{\mathscr{T}} \sum_{\omega_{1}, \omega_{2}} A\left[\omega_{1}\right] B\left[\omega_{2}\right] \delta\left[\omega_{1}+\omega_{2}\right] \\
& =\Delta \nu \sum_{\omega} A^{*}[\omega] B[\omega] \rightarrow \int d \nu A^{*}(\omega) B(\omega)
\end{aligned}
$$




\section{H.2 Gaussian Noise}

Let $n[t]$ be a noise signal, and let it be represented by the vector, $n$. If the noise is Gaussian, the probability distribution for $n$ will have the following form:

$$
p(n)=\left|\sqrt{2 \pi} \underline{\sigma}_{n}\right|^{-1} e^{-\frac{1}{2} n \cdot \underline{\sigma}_{n}^{-2} \cdot n}
$$

where the matrix, $\underline{\sigma}_{n}^{2}$, is the $N \times N$ covariance matrix, and $\left|\underline{\sigma}_{n}\right|$ represents the determinant of the matrix, $\underline{\sigma}_{n}$. Let the brackets, \langle\rangle , around some quantity, $Q(n)$, denote the average value of this quantity with respect to the probability density of $n$ :

$$
\langle Q(n)\rangle \equiv \int d^{N} n p(n) Q(n)
$$

If it is the case that $\underline{\sigma}_{n}^{2}$ is diagonal in Fourier space,

$$
\begin{aligned}
b & \equiv \underline{\sigma}_{n}^{-2} \cdot a \\
B[\omega] & =\sigma_{n}^{-2}[\omega] A[\omega]
\end{aligned}
$$

then the following simplification can be made:

$$
\begin{aligned}
n \cdot \underline{\sigma}_{n}^{-2} \cdot n & =\Delta \nu \sum_{\omega} N^{*}[\omega] \sigma_{n}^{-2}[\omega] N[\omega] \\
& =2 \Delta \nu \sum_{\omega>0} N^{*}[\omega] N[\omega] \sigma_{n}^{-2}[\omega] \\
& =\sum_{\omega>0}\left(\Re[N[\omega]]^{2}+\Im[N[\omega]]^{2}\right) \frac{2 \Delta \nu}{\sigma_{n}^{2}[\omega]}
\end{aligned}
$$

where the sum is taken only over positive values of $\omega$ because the Fourier components, $N[\omega]$, for negative $\omega$ are not independent variables.

The probability distribution for $n$ may be factored into a product of independent normal distributions for each real and imaginary component in the Fourier basis. The 
variance of each component is given by:

$$
\left\langle\Re[N[\omega]]^{2}\right\rangle=\left\langle\Im[N[\omega]]^{2}\right\rangle=\frac{\sigma_{n}^{2}[\omega]}{2 \Delta \nu}
$$

The average instantaneous noise power, $\left\langle n^{2}[t]\right\rangle$, is given by:

$$
\begin{aligned}
\left\langle n^{2}[t]\right\rangle & =\left\langle\Delta \nu^{2} \sum_{\omega_{1}, \omega_{2}} N\left[\omega_{1}\right] N\left[\omega_{2}\right] e^{i\left(\omega_{1}+\omega_{2}\right) t}\right\rangle \\
& =\Delta \nu^{2} \sum_{\omega_{1}, \omega_{2}}\left\langle N\left[\omega_{1}\right] N\left[\omega_{2}\right]\right\rangle e^{i\left(\omega_{1}+\omega_{2}\right) t} \\
& =\Delta \nu \sum_{\omega} \Delta \nu\left\langle N^{*}[\omega] N[\omega]\right\rangle \\
& =\Delta \nu \sum_{\omega} P_{n}[\omega] \rightarrow \int d \nu P_{n}(\omega)
\end{aligned}
$$

Note that the average instantaneous power is time-independent. The function, $P_{n}[\omega]$ or $P_{n}(\omega)$, is the noise power contribution per unit frequency. The relation between $P_{n}[\omega]$ and $\sigma_{n}^{2}[\omega]$ is:

$$
\begin{aligned}
P_{n}[\omega] & =\Delta \nu\left\langle N^{*}[\omega] N[\omega]\right\rangle \\
& =\Delta \nu\left\langle\Re[N[\omega]]^{2}+\Im[N[\omega]]^{2}\right\rangle \\
& =2 \Delta \nu\left\langle\Re[N[\omega]]^{2}\right\rangle \\
& =\sigma_{n}^{2}[\omega]
\end{aligned}
$$

\section{H.3 Linear Curve Fitting}

Suppose a signal, $s[t]$, is a linear function of $M$ parameters. This relationship may be written in terms of signal vectors:

$$
s=\underline{r} \cdot \lambda
$$


where $s$ is an $N$-component column vector, $\underline{r}$ is an $N \times M$ matrix, and $\lambda$ is an $M$-component column vector. The transpose of $\underline{r}$, denoted by $\bar{r}$, is an $M \times N$ matrix.

Suppose the measurement, $m$, of the signal, $s$, is corrupted by some additive Gaussian noise. This may be written in terms of vectors as:

$$
m=s+n=\underline{r} \cdot \lambda+n
$$

Given some $\lambda$ as input, the probability distribution for the measured signal is simply a Gaussian with its mean equal to the noiseless signal, $s=\underline{r} \cdot \lambda$ :

$$
p(m \mid \lambda)=\left|\sqrt{2 \pi} \underline{\sigma}_{n}\right|^{-1} e^{-\frac{1}{2}(m-\underline{r} \cdot \lambda) \cdot \underline{\sigma}_{n}^{-2} \cdot(m-\underline{r} \cdot \lambda)}
$$

Given some measurement, $m$, we wish to estimate the input, $\lambda$, which caused this measured signal. A probability distribution over $\lambda$ may be calculated using Bayes' theorem:

$$
\begin{aligned}
p(\lambda, m) & =p(m \mid \lambda) p(\lambda)=p(\lambda \mid m) p(m) \\
\Rightarrow p(\lambda \mid m) & =\frac{p(m \mid \lambda) p(\lambda)}{p(m)}
\end{aligned}
$$

If all values of $\lambda$ are assumed to be equally probable in our prior distribution, then $p(\lambda)$ is a constant and:

$$
\begin{aligned}
p(\lambda \mid m) & \propto p(m \mid \lambda) \propto e^{-\frac{1}{2}(m-\underline{r} \cdot \lambda) \cdot \underline{\sigma}_{n}^{-2} \cdot(m-\underline{r} \cdot \lambda)} \\
& \propto e^{-\frac{1}{2}\left(\lambda \cdot \bar{r} \underline{\sigma}_{n}^{-2} \underline{r} \cdot \lambda-\lambda \cdot \bar{r} \underline{\sigma}_{n}^{-2} \cdot m-m \cdot \underline{\sigma}_{n}^{-2} \underline{r} \cdot \lambda\right)} \\
& \propto e^{-\frac{1}{2}(\lambda-\mu) \cdot \underline{\sigma}^{-2} \cdot(\lambda-\mu)} \\
& \propto e^{-\frac{1}{2}\left(\lambda \cdot \underline{\sigma}^{-2} \cdot \lambda-\lambda \cdot \underline{\sigma}^{-2} \cdot \mu-\mu \cdot \underline{\sigma}^{-2} \cdot \lambda\right)}
\end{aligned}
$$

Therefore, the probability distribution for the fit parameters, $\lambda$, is a Gaussian centered at mean value $\mu$. The inverse covariance matrix, $\underline{\sigma}^{-2}$, and mean, $\mu$, can be 
easily determined by matching terms linear and quadratic in $\lambda$ :

$$
\begin{aligned}
\underline{\sigma}^{-2} & =\bar{r} \underline{\sigma}_{n}^{-2} \underline{r} \\
\mu & =\underline{\sigma}^{2} \bar{r} \underline{\sigma}_{n}^{-2} \cdot m
\end{aligned}
$$

\section{H.4 Signal to Noise Ratio}

The value of $\mu$ calculated in the previous section provides the maximum likelihood estimate of the signal parameters, $\lambda$, based on a noisy measurement, $m$. Suppose the exact signal parameters are given by $\lambda_{0}$, and the exact signal is given by $s_{0}=\underline{r} \cdot \lambda_{0}$. Then $\mu$ and $s=\underline{r} \cdot \mu$ will be noisy estimates of $\lambda_{0}$ and $s_{0}$ respectively.

The mean values of $\mu$ and $s$ are given by:

$$
\begin{aligned}
\langle\mu\rangle & =\left\langle\underline{\sigma}^{2} \bar{r} \underline{\sigma}_{n}^{-2} \cdot m\right\rangle=\underline{\sigma}^{2} \bar{r} \underline{\sigma}_{n}^{-2} \cdot\langle m\rangle \\
& =\underline{\sigma}^{2} \bar{r} \underline{\sigma}_{n}^{-2} \cdot\left\langle\underline{r} \cdot \lambda_{0}+n\right\rangle \\
& =\underline{\sigma}^{2} \bar{r} \underline{\sigma}_{n}^{-2} \underline{r} \cdot \lambda_{0} \\
& =\lambda_{0} \\
& \langle s\rangle=\langle\underline{r} \cdot \mu\rangle=\underline{r} \cdot\langle\mu\rangle=s_{0}
\end{aligned}
$$

Therefore, these are unbiased estimators. The deviation of $s$ from its mean value is:

$$
s-\langle s\rangle=s-s_{0}=\underline{r} \cdot\left(\mu-\lambda_{0}\right)=\underline{r} \underline{\sigma}^{2} \bar{r} \underline{\sigma}_{n}^{-2} \cdot n
$$


The covariance matrix of $s$ is:

$$
\begin{aligned}
\underline{\sigma}_{s} & \equiv\langle(\underline{s}-\langle\underline{s}\rangle)(\bar{s}-\langle\bar{s}\rangle)\rangle=\left\langle\underline{r} \underline{\sigma}^{2} \bar{r} \underline{\sigma}_{n}^{-2} \underline{n} \bar{n} \underline{\sigma}_{n}^{-2} \underline{r} \underline{\sigma}^{2} \bar{r}\right\rangle \\
& =\underline{r} \underline{\sigma}^{2} \bar{r} \underline{\sigma}_{n}^{-2}\langle\underline{n} \bar{n}\rangle \underline{\sigma}_{n}^{-2} \underline{r} \underline{\sigma}^{2} \bar{r} \\
& =\underline{r} \underline{\sigma}^{2} \bar{r} \underline{\sigma}_{n}^{-2} \underline{\sigma}_{n}^{2} \underline{\sigma}_{n}^{-2} \underline{r} \underline{\sigma}^{2} \bar{r} \\
& =\underline{r} \underline{\sigma}^{2} \bar{r} \underline{\sigma}_{n}^{-2} \underline{r} \underline{\sigma}^{2} \bar{r} \\
& =\underline{r} \underline{\sigma}^{2} \bar{r}
\end{aligned}
$$

The mean-squared value of $s$ is given by:

$$
\begin{aligned}
\langle s \cdot s\rangle & =\langle\mu \cdot \bar{r} \underline{r} \cdot \mu\rangle=\left\langle\left(\lambda_{0}+\underline{\sigma}^{2} \bar{r} \underline{\sigma}_{n}^{-2} \cdot n\right) \cdot \bar{r} \underline{r} \cdot\left(\lambda_{0}+\underline{\sigma}^{2} \bar{r} \underline{\sigma}_{n}^{-2} \cdot n\right)\right\rangle \\
& =s_{0} \cdot s_{0}+\left\langle n \cdot \underline{\sigma}_{n}^{-2} \underline{r} \underline{\sigma}^{2} \bar{r} \underline{r} \underline{\sigma^{2}} \bar{r} \underline{\sigma}_{n}^{-2} \cdot n\right\rangle \\
& =\langle s\rangle \cdot\langle s\rangle+\left\langle\operatorname{Tr}\left(\underline{n} \bar{n} \underline{\sigma}_{n}^{-2} \underline{r} \underline{\sigma}^{2} \bar{r} \underline{r} \underline{\sigma}^{2} \bar{r} \underline{\sigma}_{n}^{-2}\right)\right\rangle \\
& =\langle s\rangle \cdot\langle s\rangle+\operatorname{Tr}\left(\langle\underline{n} \bar{n}\rangle \underline{\sigma}_{n}^{-2} \underline{r} \underline{\sigma}^{2} \bar{r} \underline{r} \underline{\sigma}^{2} \bar{r} \underline{\sigma}_{n}^{-2}\right) \\
& =\langle s\rangle \cdot\langle s\rangle+\operatorname{Tr}\left(\underline{\sigma}_{n}^{2} \underline{\sigma}_{n}^{-2} \underline{r} \underline{\sigma}^{2} \bar{r} \underline{r} \underline{\sigma}^{2} \bar{r} \underline{\sigma}_{n}^{-2}\right) \\
& =\langle s\rangle \cdot\langle s\rangle+\operatorname{Tr}\left(\underline{r} \underline{\sigma}^{2} \bar{r} \underline{r} \underline{\sigma}^{2} \bar{r} \underline{\sigma}_{n}^{-2}\right) \\
& =\langle s\rangle \cdot\langle s\rangle+\operatorname{Tr}\left(\underline{\sigma}^{2} \bar{r} \underline{r} \underline{\sigma}^{2} \bar{r} \underline{\sigma}_{n}^{-2} \underline{r}\right) \\
& =\langle s\rangle \cdot\langle s\rangle+\operatorname{Tr}\left(\underline{\sigma}^{2} \bar{r} \underline{r}\right)
\end{aligned}
$$

Therefore, the variance of $s$ is given by:

$$
\langle s \cdot s\rangle-\langle s\rangle \cdot\langle s\rangle=\operatorname{Tr}\left(\underline{\sigma}^{2} \bar{r} \underline{r}\right)
$$

The signal to noise ratio is defined to be the ratio of the square of the mean of $s$ to the variance of $s$ :

$$
\mathrm{SNR}=\frac{\langle s\rangle \cdot\langle s\rangle}{\langle s \cdot s\rangle-\langle s\rangle \cdot\langle s\rangle}=\frac{s_{0} \cdot s_{0}}{\operatorname{Tr}\left(\underline{\sigma}^{2} \bar{r} \underline{r}\right)}
$$

The quantity, $s_{0} \cdot s_{0}$, is the energy contained in the signal, $\mathscr{E}_{s}$. Therefore, the 
signal to noise ratio is equal to the signal energy divided by the noise energy:

$$
\mathrm{SNR}=\frac{\mathscr{E}_{s}}{\mathscr{E}_{n}}
$$

where the noise energy is given by:

$$
\mathscr{E}_{n}=\operatorname{Tr}\left(\underline{\sigma}^{2} \bar{r} \underline{r}\right)
$$

\section{H.5 Fits with Only One Parameter}

For a fit with only one parameter, the matrix, $\underline{r}$, is an $N$-component column vector, $r$. The matrix $\bar{r} \underline{r}$ has only one component, and is simply equal to $r \cdot r$. Likewise, the matrix, $\underline{\sigma}^{-2}$, has only one component, and is equal to $r \cdot \underline{\sigma}_{n}^{-2} \cdot r$. Using these values, the noise energy is given by:

$$
\mathscr{E}_{n}=\operatorname{Tr}\left(\underline{\sigma}^{2} \bar{r} \underline{r}\right)=\frac{r \cdot r}{r \cdot \underline{\sigma}_{n}^{-2} \cdot r}
$$

If $\underline{\sigma}_{n}$ is diagonal in Fourier space, then, using the previously derived results, the noise energy may be rewritten as:

$$
\mathscr{E}_{n}=\frac{\Delta \nu \sum_{\omega} R^{*}[\omega] R[\omega]}{\Delta \nu \sum_{\omega} R^{*}[\omega] R[\omega] \sigma_{n}^{-2}[\omega]}=\frac{\Delta \nu \sum_{\omega} R^{*}[\omega] R[\omega]}{\Delta \nu \sum_{\omega} \frac{R^{*}[\omega] R[\omega]}{P_{n}[\omega]}}
$$

In the continuum limit, the noise energy may be rewritten as:

$$
\mathscr{E}_{n}=\frac{\int d \nu R^{*}(\omega) R(\omega)}{\int d \nu \frac{R^{*}(\omega) R(\omega)}{P_{n}(\omega)}}
$$

If the noise spectral power density, $P_{n}(\omega)$, is constant in all regions of frequency space in which $R(\omega)$ is non-zero, then $P_{n}(\omega)=P_{n}$ may be factored out of the integral in the denominator, and the noise energy is given by:

$$
\mathscr{E}_{n}=P_{n}
$$




\section{Appendix I}

\section{Piezoelectric Resonators}

This section provides a general analysis of the electrical and mechanical properties of a piezoelectric resonator. The device under consideration consists of two electrodes connected to a piezoelectric material. The material has natural modes of vibration determined by its mass density and elasticity. It will be assumed in this paper that the resonances are sharp, i.e. that they all have a high $Q$. It will also be assumed in this paper that all of the mechanical and electrical properties of the piezo are linear.

\section{I.1 The Elastic Lagrangian of the Piezo Actuator}

Assume the displacement field of the piezo resonator is $u(x)$. The kinetic energy density is $1 / 2 \rho \dot{u}^{2}$, where $\rho$ is the mass density of the piezo, and the elastic potential energy density is $1 / 2 \underline{s} \cdot \underline{\underline{K}} \cdot \underline{s}$, where $\underline{\underline{K}}$ is the elasticity tensor, and $\underline{s}$ is the strain tensor, defined by:

$$
\underline{s}=\frac{1}{2}(\underline{\nabla} \bar{u}+\underline{u} \bar{\nabla})
$$

In general, both $\rho$ and $\underline{\underline{K}}$ may be functions of position.

The stress tensor, $\underline{\sigma}$, is related to the strain tensor by the following equation:

$$
\underline{\sigma}=\underline{\underline{K}} \cdot \underline{s}
$$


The total kinetic energy is given by:

$$
T=\int d \mathcal{V} \frac{1}{2} \rho \dot{u}^{2}
$$

and the total potential energy is given by:

$$
\begin{aligned}
U & =\int d \mathcal{V} \frac{1}{2} \underline{s} \cdot \underline{\underline{K}} \cdot \underline{s}=\int d \mathcal{V} \frac{1}{2}(\underline{u} \bar{\nabla}) \cdot \underline{\underline{K}} \cdot \underline{s}=\int d \mathcal{V} \frac{1}{2}(\underline{u} \bar{\nabla}) \cdot \underline{\sigma} \\
& =\int d \mathcal{V} \frac{1}{2} \dot{\nabla} \cdot \underline{\sigma} \cdot \dot{u}=-\int d \mathcal{V} \frac{1}{2}(\dot{\nabla} \cdot \underline{\underline{\sigma}}) \cdot u+\oint \frac{1}{2} d \mathcal{A} \cdot \underline{\sigma} \cdot u
\end{aligned}
$$

The surface term in equation I.4 is zero because the boundary conditions on the resonator are either Neumann, in which case $\underline{\sigma} \cdot d \mathcal{A}=0$ on the boundary, or else Dirichlet, in which case $u=0$ on the boundary. Therefore, the potential energy is:

$$
U=-\int d \mathcal{V} \frac{1}{2} u \cdot(\underline{\hat{\sigma}} \cdot \dot{\nabla})
$$

These equations may be rewritten using operator notation:

$$
\begin{aligned}
T & =\frac{1}{2} \dot{u} * \hat{M} * \dot{u} \\
U & =\frac{1}{2} u * \hat{K} * u
\end{aligned}
$$

where the $*$ symbol denotes an inner product between two functions, defined by:

$$
u * v \equiv \int d \mathcal{V} u(x) \cdot v(x)
$$

The $\hat{M}$ and $\hat{K}$ operators are defined by:

$$
\begin{aligned}
\hat{M} * u & \equiv \rho u \\
\hat{K} * u & \equiv-\underline{\hat{\sigma}} \cdot \dot{\nabla}=-(\underline{\underline{K}} \cdot(\underline{\nabla} \bar{u})) \cdot \dot{\nabla}
\end{aligned}
$$

Both of these operators are symmetric, and in terms of them the elastic Lagrangian 
is:

$$
\mathscr{L}_{E l}=\frac{1}{2} \dot{u} * \hat{M} * \dot{u}-\frac{1}{2} u * \hat{K} * u
$$

The Euler-Lagrange equation of motion is:

$$
\hat{M} * \ddot{u}=-\hat{K} * u
$$

Define the field, $v \equiv \hat{M}^{1 / 2} * u$. In terms of $v$ the Euler-Lagrange equation is:

$$
\ddot{v}=-\hat{M}^{-1 / 2} \hat{K} \hat{M}^{-1 / 2} * v=-\hat{\Omega}^{2} * v
$$

Where the symmetric operator, $\hat{\Omega}^{2}$, is defined to be equal to $\hat{M}^{-1 / 2} \hat{K} \hat{M}^{-1 / 2}$. Because $\hat{\Omega}$ is symmetric, all of its eigenvalues are real. Define $v_{n}$ to be the $n^{\text {th }}$ eigenfunction of $\hat{\Omega}$ with eigenvalue $\omega_{n}$, and define $f_{n}$ to be:

$$
f_{n} \equiv \hat{M}^{-1 / 2} * v_{n}
$$

Because all the $v_{n}$ functions are orthogonal, the $f_{n}$ functions have the properties:

$$
\begin{gathered}
f_{n} * \hat{M} * f_{m}=v_{n} * v_{m}=0 \quad \text { for } n \neq m \\
\text { and } \\
f_{n} * \hat{K} * f_{m}=v_{n} * \hat{M}^{-1 / 2} \hat{K} \hat{M}^{-1 / 2} * v_{m}=v_{n} * \hat{\Omega}^{2} * v_{m}=0 \quad \text { for } n \neq m
\end{gathered}
$$

The functions, $f_{n}$, are the modes of vibration of the resonator. Because the scale of each eigenfunction is arbitrary, let the $f_{n}$ be defined to be dimensionless, and let the displacement field, $u$, be decomposed in terms of these modes and a set of generalized coordinates, $q_{n}$, with units of length:

$$
u(x)=\sum_{n} q_{n} f_{n}(x)
$$


In terms of the $q_{n}$, the elastic Lagrangian is:

$$
\mathscr{L}_{E l}=\sum_{n}\left(\frac{1}{2} m_{n} \dot{q}_{n}^{2}-\frac{1}{2} k_{n} q_{n}^{2}\right)
$$

where the $m_{n}$ and $k_{n}$ coefficients are defined by:

$$
\begin{aligned}
m_{n} & \equiv f_{n} * \hat{M} * f_{n} \\
k_{n} & \equiv f_{n} * \hat{K} * f_{n}
\end{aligned}
$$

From the definition of these eigenfunctions, it also follows that $\omega_{n}=\sqrt{k_{n} / m_{n}}$.

For future use, let the dimensionless strain of each mode, $\underline{f}_{n}$, be defined by the equation:

$$
\underline{\mathrm{f}}_{n} \equiv \frac{1}{2}\left(\underline{\nabla} \bar{f}_{n}+\underline{f}_{n} \bar{\nabla}\right)
$$

The strain, $\underline{s}$, is therefore equal to:

$$
\underline{s}=\sum_{n} q_{n} \underline{f}_{n}
$$

\section{I.2 Electromagnetic Lagrangian and Coupling}

The Electromagnetic Lagrangian density is:

$$
\mathcal{L}_{E M}=\frac{1}{2} E \cdot \underline{\epsilon} \cdot E+\frac{1}{2} B \cdot \underline{\mu}^{-1} \cdot B
$$

where:

$$
E=-\nabla \phi-\dot{A} \quad, \quad B=\nabla \wedge A
$$

The charge and current source term is:

$$
\mathcal{L}_{S t o t}=-\rho_{\text {tot }} \phi+J_{\text {tot }} \cdot A
$$

The total charge and current densities, $\rho_{t o t}$ and $J_{t o t}$, are assumed to be the sum of a free charge and current density, $\rho$ and $J$, and a charge and current density, $\rho_{s}$ and 
$J_{s}$, generated by a strain-induced polarization, $P_{s}$, where $\rho_{s}=-\nabla \cdot P_{s}$ and $J_{s}=\dot{P}_{s}$. The polarization is a linear function of the strain tensor, $\underline{s}$ :

$$
P_{s}=\underline{\underline{\mathrm{e}}} \cdot \underline{s}
$$

where $\underline{\underline{e}}$ is the piezoelectric tensor. Up to a total derivative, the source term involving the polarization may be rewritten in terms of the electric field:

$$
\begin{aligned}
\mathcal{L}_{C} & \equiv-\rho_{s} \phi+J_{s} \cdot A=\phi \nabla \cdot P_{s}+A \cdot \dot{P}_{s} \\
& =-P_{s} \cdot \nabla \phi-P_{s} \cdot \dot{A} \\
& =P_{s} \cdot E \\
& =E \cdot \underline{\underline{\mathrm{e}}} \cdot \underline{s}
\end{aligned}
$$

This term in the Lagrangian represents the coupling between the electrical and mechanical systems. The remaining source term is:

$$
\mathcal{L}_{S} \equiv-\rho \phi+J \cdot A
$$

and

$$
\mathcal{L}_{S t o t}=\mathcal{L}_{S}+\mathcal{L}_{C}
$$

When a voltage is applied to the electrodes of the piezo resonator, an electric field is generated which is linearly proportional to the voltage:

$$
E=V g
$$

where $g$ is a vector field with units of inverse length. In terms of this field, the coupling term may be rewritten in terms of the potential and the generalized coordinates, $q_{n}$ :

$$
\mathscr{L}_{C}=\int d \mathcal{V} E \cdot \underline{\underline{\mathrm{e}}} \cdot \underline{\underline{s}}=\sum_{n} \int d \mathcal{V} V q_{n} g \cdot \underline{\underline{\mathrm{e}}} \cdot \underline{\mathrm{f}}_{n}=\sum_{n} k_{n} \alpha_{n} q_{n} V
$$


where $\alpha_{n}$ is defined to be:

$$
\alpha_{n} \equiv \frac{1}{k_{n}} \int d \mathcal{V} g \cdot \underline{\underline{\mathrm{e}}} \cdot \underline{\mathrm{f}}_{n}
$$

\section{I.3 The Lagrangian and the Euler-Lagrange Equa- tions}

The full Lagrangian is:

$$
\begin{aligned}
\mathscr{L}= & \mathscr{L}_{E l}+\mathscr{L}_{C}+\mathscr{L}_{E M}+\mathscr{L}_{S} \\
= & \sum_{n}\left(\frac{1}{2} m_{n} \dot{q}_{n}^{2}-\frac{1}{2} k_{n} q_{n}^{2}+k_{n} \alpha_{n} q_{n} V\right) \\
& +\int d \mathcal{V}\left(\frac{1}{2} E \cdot \underline{\epsilon} \cdot E+\frac{1}{2} B \cdot \underline{\mu}^{-1} \cdot B-\rho \phi+J \cdot A\right)
\end{aligned}
$$

The $D$ and $H$ fields are defined to be:

$$
D=\underline{\epsilon} \cdot E+P_{s} \quad, \quad H=\underline{\mu}^{-1} \cdot B
$$

In terms of these fields, Maxwell's Equations are:

$$
\begin{aligned}
\nabla \wedge B & =0 \\
\nabla \wedge E & =-\dot{B} \\
\nabla \cdot D & =\rho \\
\nabla \cdot H & =-J-\dot{D}
\end{aligned}
$$

The Euler-Lagrange equations for the vibrational modes are:

$$
\begin{aligned}
m_{n} \ddot{q}_{n} & =-m_{n} \omega_{n}^{2} q_{n}+m_{n} \omega_{n}^{2} \alpha_{n} V \\
\ddot{q}_{n}+\omega_{n}^{2} q_{n} & =\omega_{n}^{2} \alpha_{n} V
\end{aligned}
$$

For frequencies far below resonance, the solution to this equation is $q_{n}=\alpha_{n} V$. The 
electromechanical coupling coefficient, $\alpha_{n}$, is therefore equal to the deflection of mode $n$ per volt.

\section{I.4 Induced Charge}

According to Gauss' Law:

$$
\begin{gathered}
\nabla \cdot D=\nabla \cdot\left(\underline{\epsilon} \cdot E+P_{s}\right)=-\nabla \cdot \underline{\epsilon} \cdot \nabla \phi+\nabla \cdot P_{s}=\rho \\
\text { or } \\
-\nabla \cdot \underline{\epsilon} \cdot \nabla \phi=\rho_{s}+\rho=\rho_{\text {tot }}
\end{gathered}
$$

Because $\nabla \cdot \underline{\epsilon} \cdot \nabla$ is a symmetric operator, the reciprocity theorem holds. For any two separate configurations of charge, $\rho_{1}$ and $\rho_{2}$, and potentials, $\phi_{1}$ and $\phi_{2}$, which are solutions to equation I.37, the following equation is satisfied:

$$
\int d \mathcal{V} \phi_{1} \rho_{2}=\int d \mathcal{V} \phi_{2} \rho_{1}
$$

Consider the following two charge configurations:

(1) Let a voltage $+V_{1} / 2$ be applied to one electrode and a voltage $-V_{1} / 2$ be applied to the other. ${ }^{1}$ Assume the piezo strain is zero, such that $\rho_{s}=0$. The free charge, $\rho$, will also be zero everywhere except on the surfaces of the electrodes. The electrodes will be surrounded by a potential, $\phi_{1}=r V_{1}$, where $r$ is the dimensionless Ramo potential. The electric field, $E_{1}$, is equal to $-\nabla \phi_{1}=-\nabla r V_{1}=g V_{1}$, and therefore $g=-\nabla r$.

The first electrode will have charge $Q_{1}=C V_{1}$, where $C$ is the capacitance, and the second electrode will have charge $-Q_{1}$. The stored energy is $\mathscr{E}=1 / 2 Q_{1} V_{1}=1 / 2 C V_{1}^{2}$. In terms of $g$ :

$$
\mathscr{E}=\int d \mathcal{V} \frac{1}{2} \rho_{1} \phi_{1}=\int d \mathcal{V} \frac{1}{2} E_{1} \cdot \underline{\epsilon} \cdot E_{1}=\frac{1}{2} \int d \mathcal{V} g \cdot \underline{\epsilon} \cdot g V_{1}^{2}
$$

\footnotetext{
${ }^{1}$ The electrodes' capacitance to infinity is assumed to be negligible.
} 
Therefore, the capacitance is given by the equation:

$$
C=\int d \mathcal{V} g \cdot \underline{\epsilon} \cdot g
$$

(2) Now assume both electrodes are held at ground potential, and a strain is applied to the piezo generating a charge density, $\rho_{s}$, and inducing a charge $Q_{2}$ and $-Q_{2}$ on the first and second electrodes respectively.

The reciprocity theorem may be applied to this pair of charge configurations and potentials. Because $\phi_{2}$ is zero in all locations where $\rho_{1}$ is nonzero, the right hand side of equation I.38 is zero. The left hand side is equal to:

$$
\int d \mathcal{V} \phi_{1} \rho_{2}=\int d \mathcal{V} r V \rho_{s}+V Q_{2}=0
$$

Therefore, the induced charge, $Q_{2}$, is:

$$
\begin{aligned}
Q_{2} & =-\int d \mathcal{V} r \rho_{s}=\int d \mathcal{V} r \nabla \cdot P_{s}=-\int d \mathcal{V} \nabla r \cdot P_{s}=\int d \mathcal{V} g \cdot P_{s} \\
& =\int d \mathcal{V} g \cdot \underline{\underline{\mathrm{e}}} \cdot \underline{s}=\sum_{n} \int d \mathcal{V} g \cdot \underline{\underline{\mathrm{e}}} \cdot \underline{\mathrm{f}}_{n} q_{n}=\sum_{n} k_{n} \alpha_{n} q_{n}
\end{aligned}
$$

Due to the linearity of Maxwell's Equations, the total charge, $Q_{\text {ind }}$, induced by both an applied voltage and a strain is the sum of the charges in these two configurations:

$$
Q_{i n d}=C V+\sum_{n} k_{n} \alpha_{n} q_{n}
$$

\section{I.5 Dissipation and Impedance}

The Euler-Lagrange equations do not include the effects of dissipation. These effects may be modeled by including a linear damping term in the equations of motion for the vibrational modes:

$$
\ddot{q}_{n}+\gamma_{n} \dot{q}_{n}+\omega_{n}^{2} q_{n}=\alpha_{n} \omega_{n}^{2} V
$$


In frequency space, this equation has the solution:

$$
\begin{gathered}
\left(-\omega^{2}+i \omega \gamma_{n}+\omega_{n}^{2}\right) q_{n}=\alpha_{n} \omega_{n}^{2} V \\
q_{n}=\frac{\alpha_{n} \omega_{n}^{2}}{-\omega^{2}+i \omega \gamma_{n}+\omega_{n}^{2}} V
\end{gathered}
$$

The current is the time derivative of the charge:

$$
I=\dot{Q}_{i n d}=i \omega C V+\sum_{n} i \omega \frac{m_{n} \alpha_{n}^{2} \omega_{n}^{4}}{-\omega^{2}+i \omega \gamma_{n}+\omega_{n}^{2}} V
$$

Therefore, the input admittance of the piezo resonator is:

$$
\begin{aligned}
Y & =i \omega C+\sum_{n} i \omega \frac{m_{n} \alpha_{n}^{2} \omega_{n}^{4}}{-\omega^{2}+i \omega \gamma_{n}+\omega_{n}^{2}}=i \omega C+\sum_{n} \frac{m_{n} \alpha_{n}^{2} \omega_{n}^{4}}{i \omega+\gamma_{n}+\frac{\omega_{n}^{2}}{i \omega}} \\
& =i \omega C+\sum_{n} \frac{1}{i \omega L_{n}+R_{n}+\frac{1}{i \omega C_{n}}}
\end{aligned}
$$

This impedance is electrically equivalent to one series RLC circuit for each mode in parallel with the intrinsic capacitance of the piezo. The RLC values are given by:

$$
\begin{aligned}
L_{n} & =\frac{1}{m_{n} \alpha_{n}^{2} \omega_{n}^{4}} \\
C_{n} & =m_{n} \alpha_{n}^{2} \omega_{n}^{2} \\
R_{n} & =\frac{\gamma_{n}}{m_{n} \alpha_{n}^{2} \omega_{n}^{4}}
\end{aligned}
$$

The RLC circuits may equivalently be described by their impedances, $Z_{n}=$ $\sqrt{L_{n} / C_{n}}$, resonant frequencies, $\omega_{n}=1 / \sqrt{L_{n} C_{n}}$, and quality factors, $Q_{n}=Z_{n} / R_{n}$, with:

$$
\begin{aligned}
Z_{n} & =\frac{1}{m_{n} \alpha_{n}^{2} \omega_{n}^{3}} \\
Q_{n} & =\frac{\omega_{n}}{\gamma_{n}}
\end{aligned}
$$




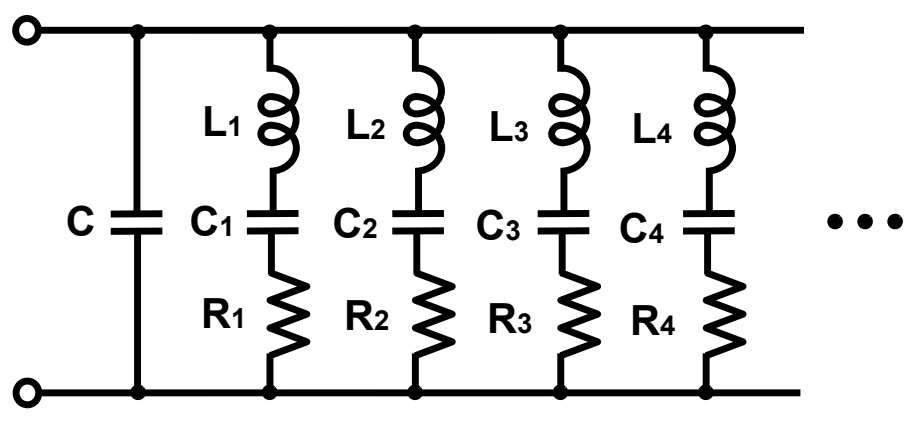

Figure I.1: An equivalent circuit for the electrical behavior of the piezo resonator.

The RLC values are related to these by the following equations:

$$
\begin{aligned}
L_{n} & =\frac{Z_{n}}{\omega_{n}} \\
C_{n} & =\frac{1}{\omega_{n} Z_{n}} \\
R_{n} & =\frac{Z_{n}}{Q_{n}}
\end{aligned}
$$

\section{I.6 Piezo Properties and Electrically Measurable Quantities}

The following tables list the properties of the piezoelectric material and the piezo resonator, as well as whether they vary with temperature.

The functions, $f_{i}$, are eigenfunctions of the elastic wave equation. If $\underline{\underline{K}}$ only varies in magnitude, then the $f_{i}$ should be temperature-independent. Likewise, $g$ depends only on the electrode geometry and on $\underline{\epsilon}$. If $\underline{\epsilon}$ only varies in magnitude, then $g$ is also temperature-independent. The invariance of $f_{i}$ and $g$ with temperature may still hold for certain geometries of the resonator and electrodes, even if the components of the elasticity and permittivity tensors change independently, as long as there are no near-degeneracies in the eigenvalues of $\hat{\Omega}$.

The electrically-measurable properties of the piezo resonator are $\omega_{n}, Q_{n}, Z_{n}$, and 
Table I.1: Piezoelectric Material Properties

\begin{tabular}{|c|c|c|}
\hline Property & Symbol & Variable \\
\hline \hline Mass Density & $\rho$ & no \\
\hline Elasticity Tensor & $\underline{\underline{K}}$ & yes \\
\hline Piezoelectric Tensor & $\underline{\underline{\mathrm{e}}}$ & yes \\
\hline Permittivity Tensor & $\underline{\epsilon}$ & yes \\
\hline
\end{tabular}

Table I.2: Resonator Properties

\begin{tabular}{|c|c|c|c|}
\hline Property & Symbol & Value & Variable \\
\hline \hline Effective Mass & $m_{n}$ & $\int d \mathcal{V} \rho f_{n}^{2}$ & no \\
\hline Stiffness & $k_{n}$ & $\int d \mathcal{V} \underline{\underline{f}}_{n} \cdot \underline{\underline{K}} \cdot \underline{\underline{f}}_{n}$ & yes \\
\hline Coupling & $\alpha_{n}$ & $\int d \mathcal{V} g \cdot \underline{\underline{\mathrm{e}}} \cdot \underline{\underline{f}}_{n} / k_{n}$ & yes \\
\hline Capacitance & $C$ & $\int d \mathcal{V} g \cdot \underline{\epsilon} \cdot g$ & yes \\
\hline
\end{tabular}

$C$. The resonator properties, $k_{n}$ and $\alpha_{n}$, may be derived from the electrically measurable properties and from the effective mass, $m_{n}$, which is temperature-independent:

$$
\begin{aligned}
k_{n} & =m_{n} \omega_{n}^{2} \\
\alpha_{n} & =\frac{1}{\sqrt{m_{n} Z_{n} \omega_{n}^{3}}}
\end{aligned}
$$




\section{Appendix $\mathbf{J}$}

\section{Matlab Code}

The following sections contain the Matlab code for the main functions used in executing a raster scan. The primary function is Scan.m, which first defines file names for saving data, calls the initialization script, setup.m, and reads in the Points.csv file which defines the points in the raster scan pattern. Setup.m reads the initialization file, Setup.ini, which contains parameters which control the behavior of the various functions, and which can be changed from scan to scan. Noise traces are then acquired for channels 1 and 2, and the function Neutralize.m is called to neutralize the crystal before entering the for-loop, which executes the raster scan and records the pulse traces, temperature, and time for each point. Finally, the data is saved to a .mat file, and copied to a back-up disk and a server, where the raw data is processed and converted into a two-dimensional charge density map.

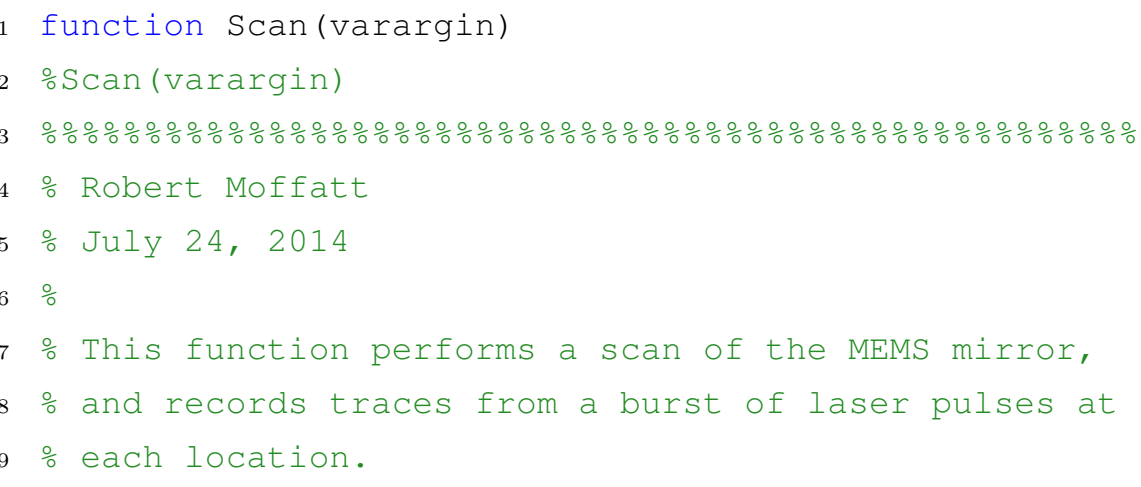




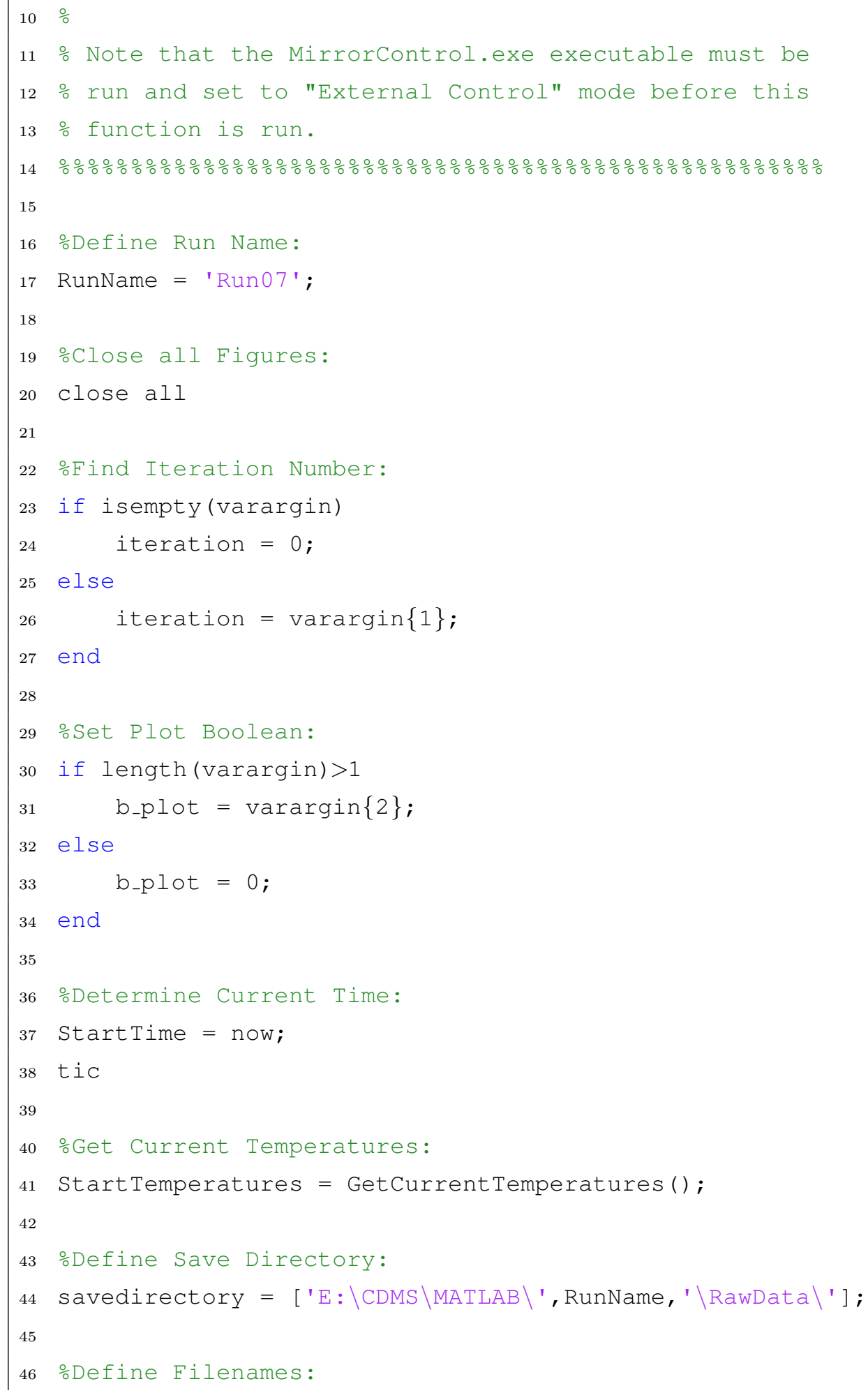




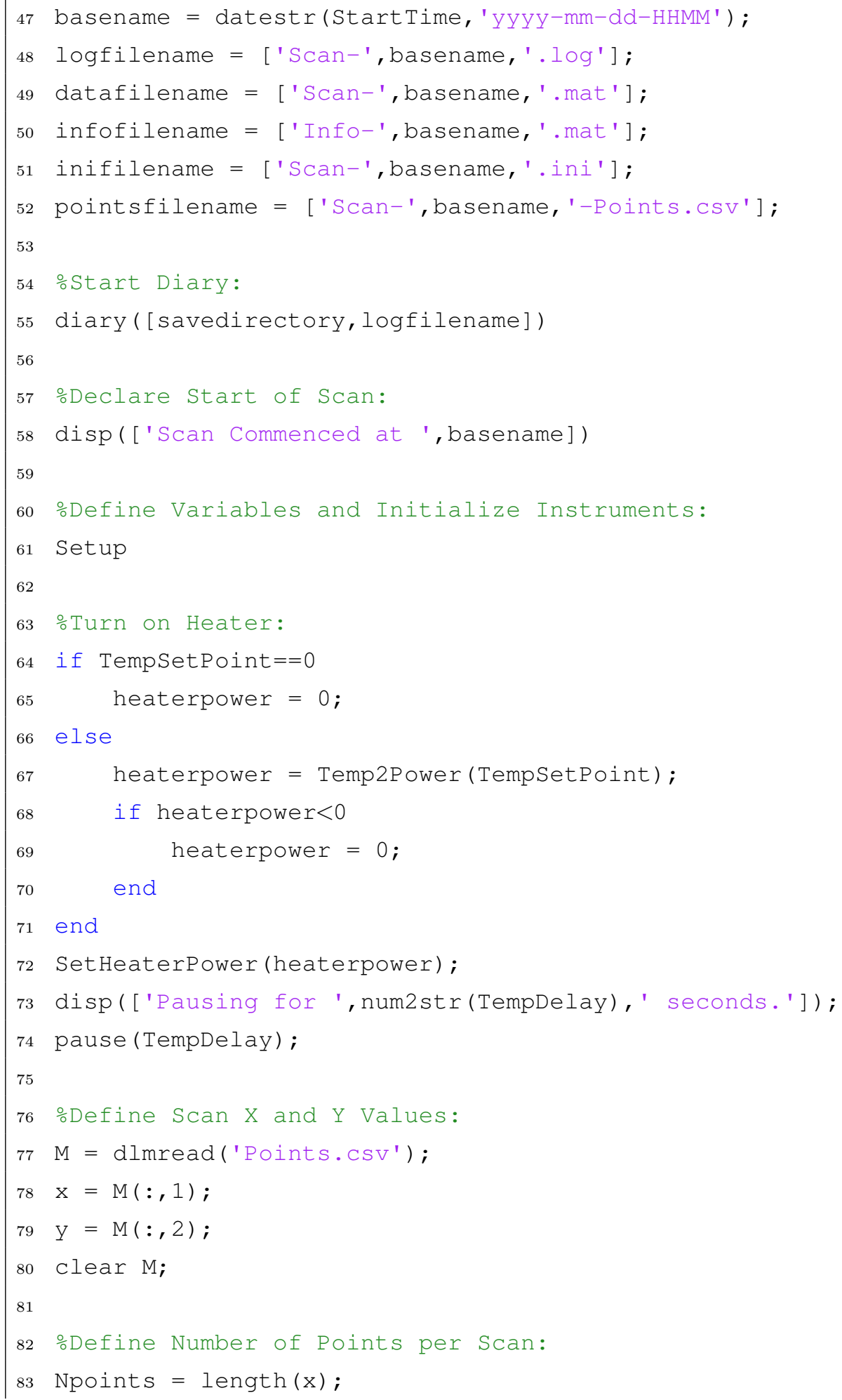




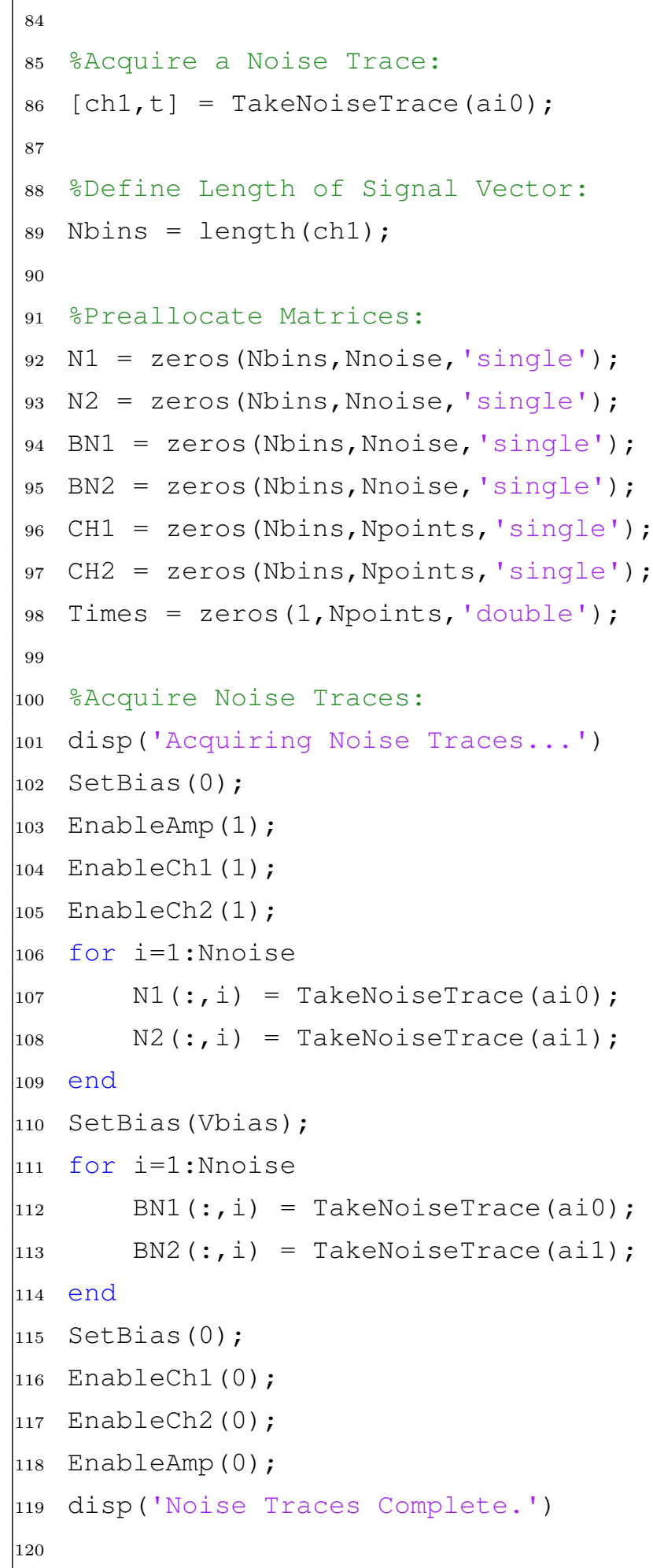




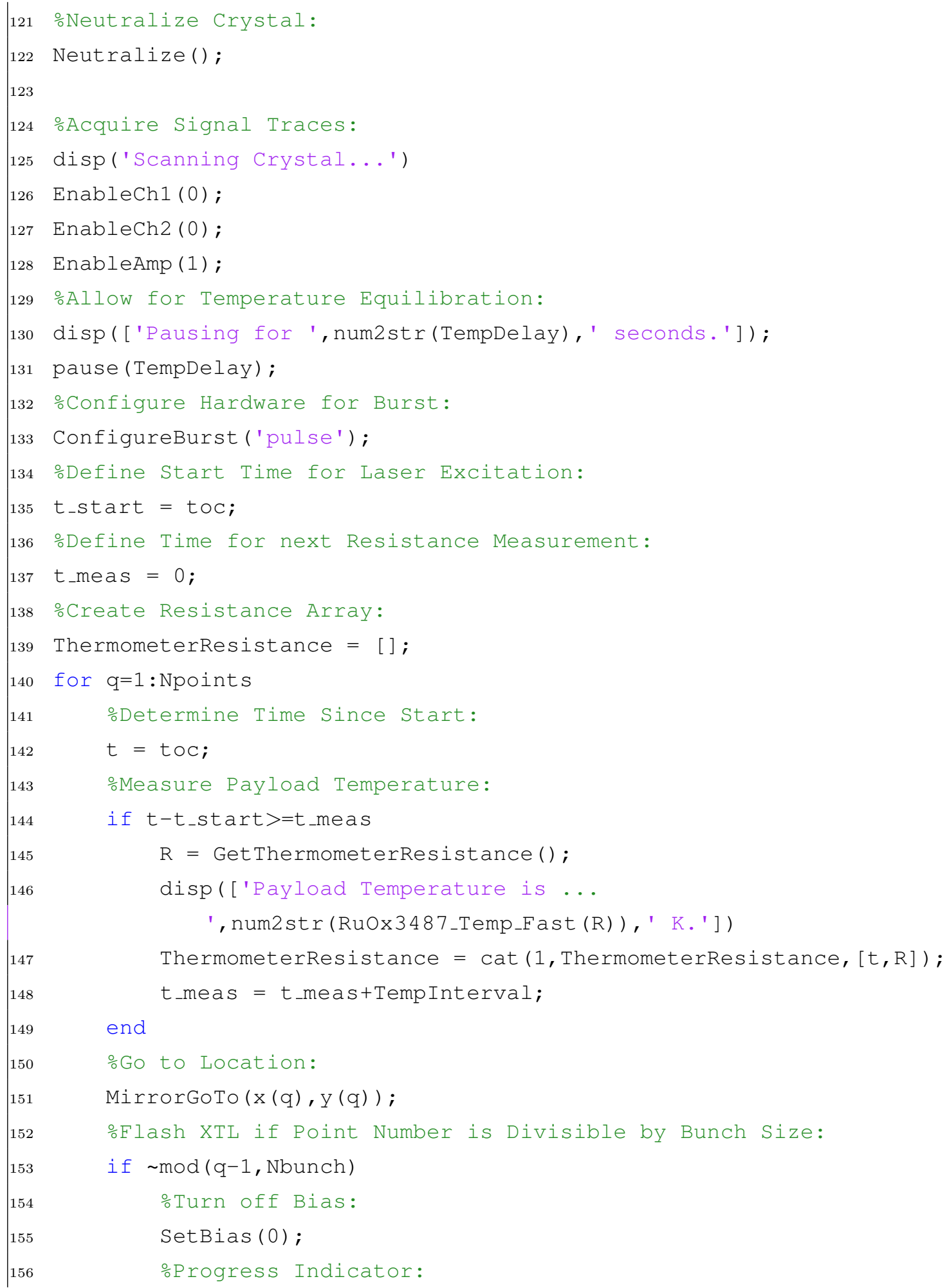




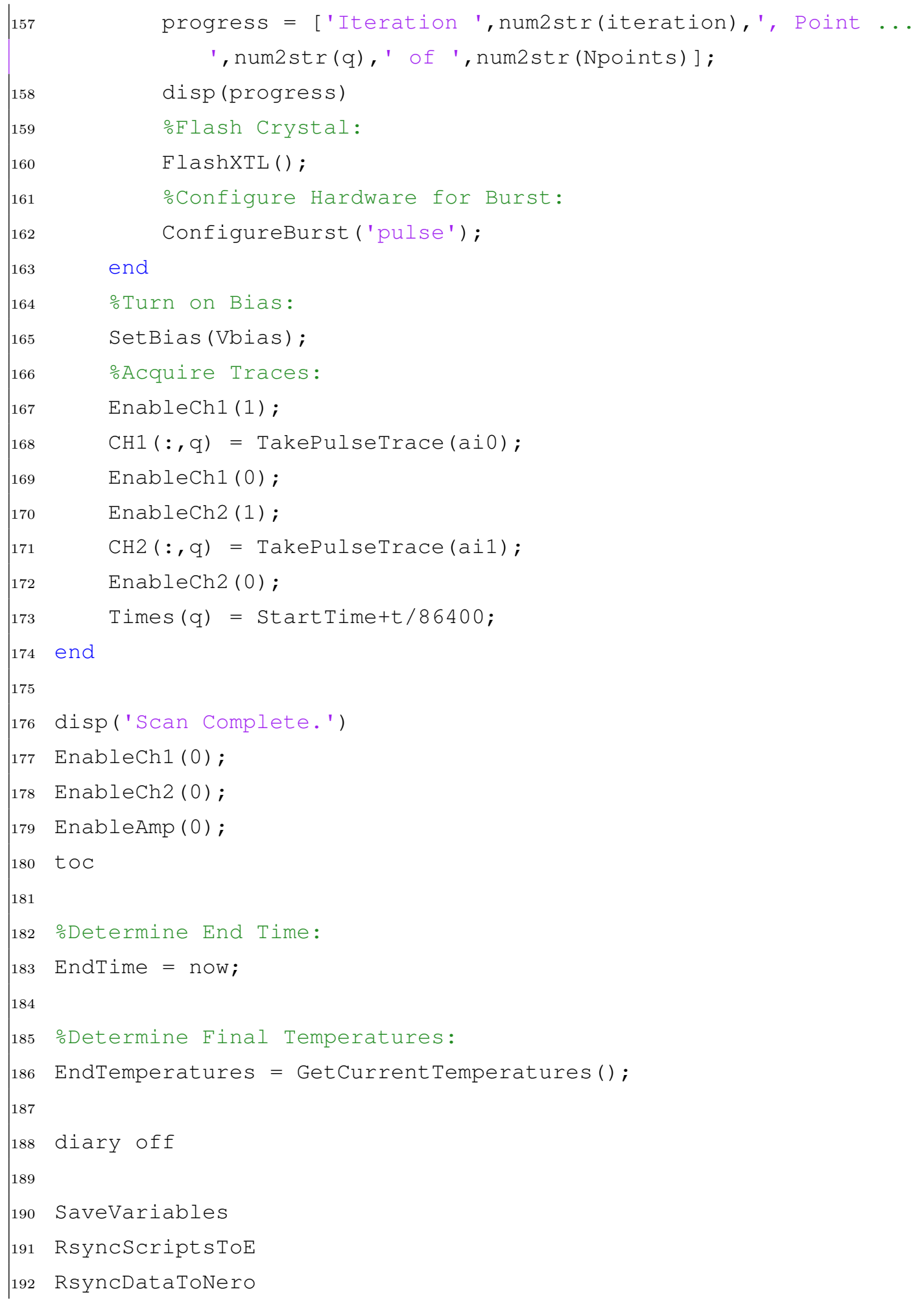




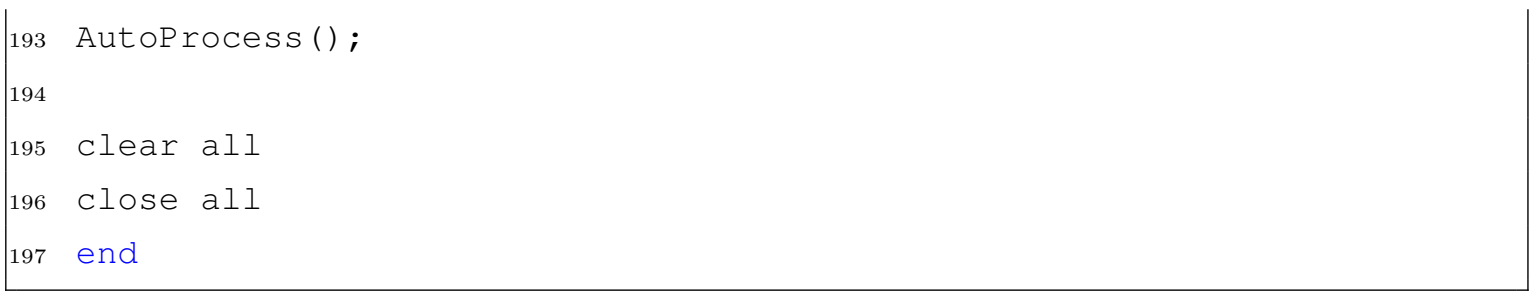

\section{Setup.m:}

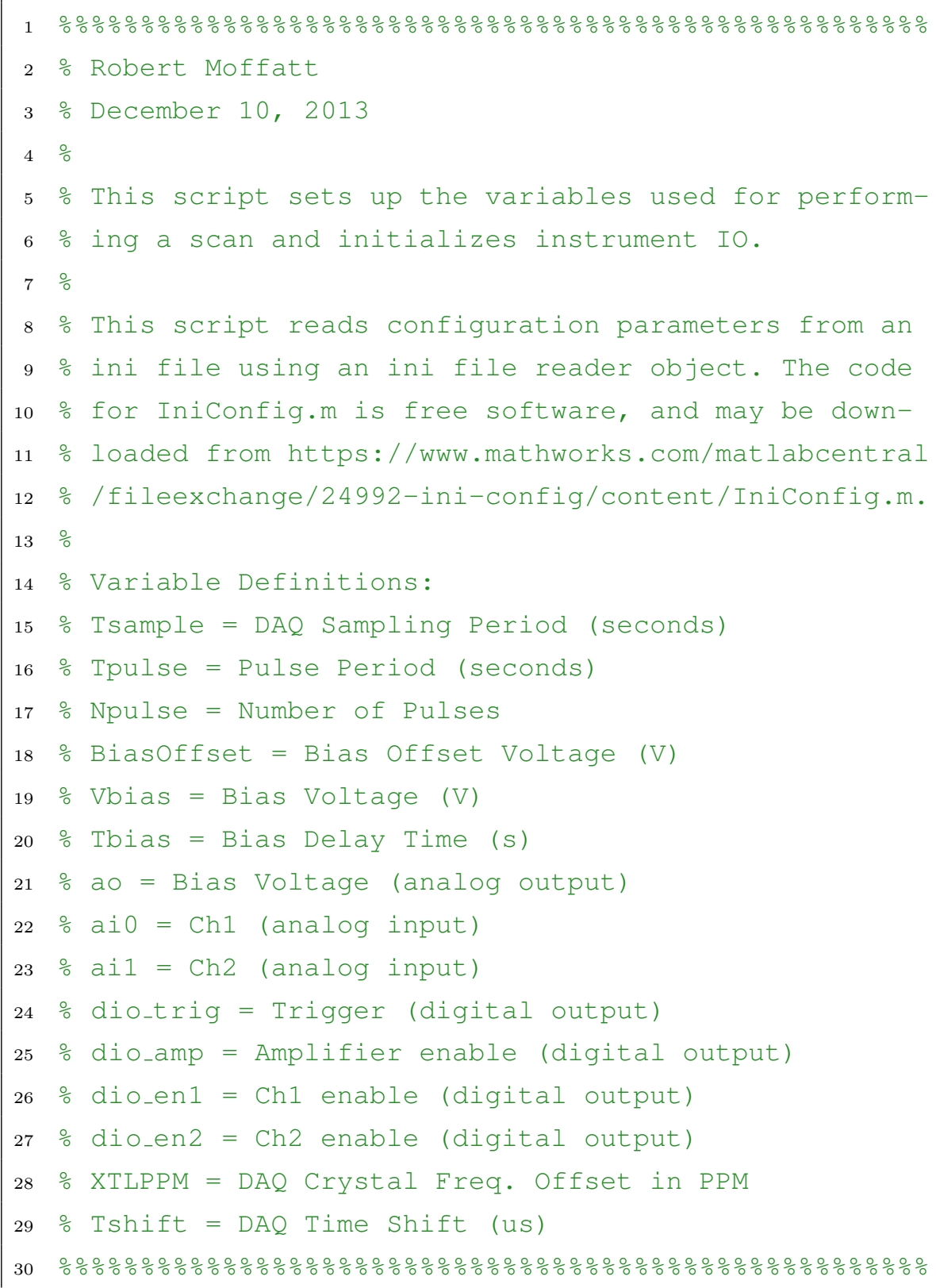




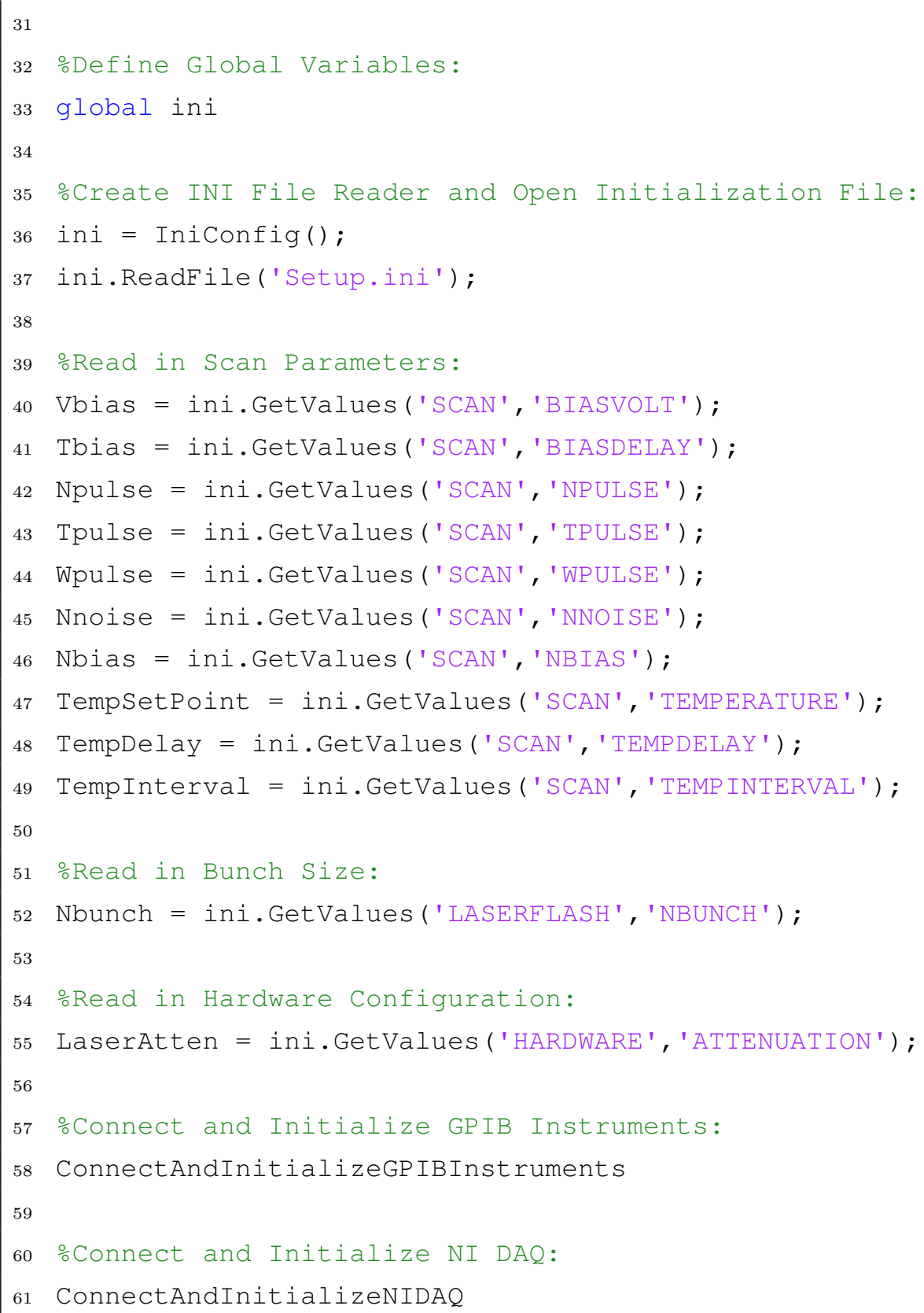

Setup.ini:

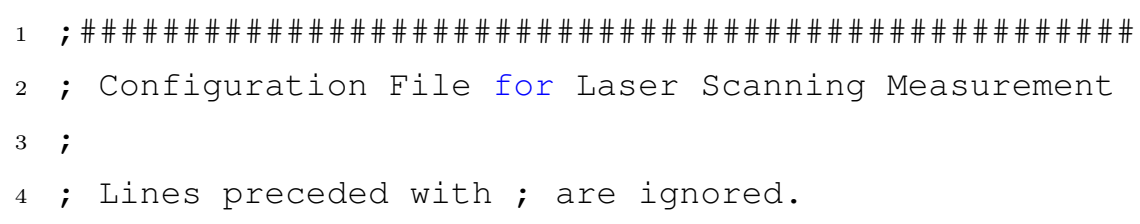




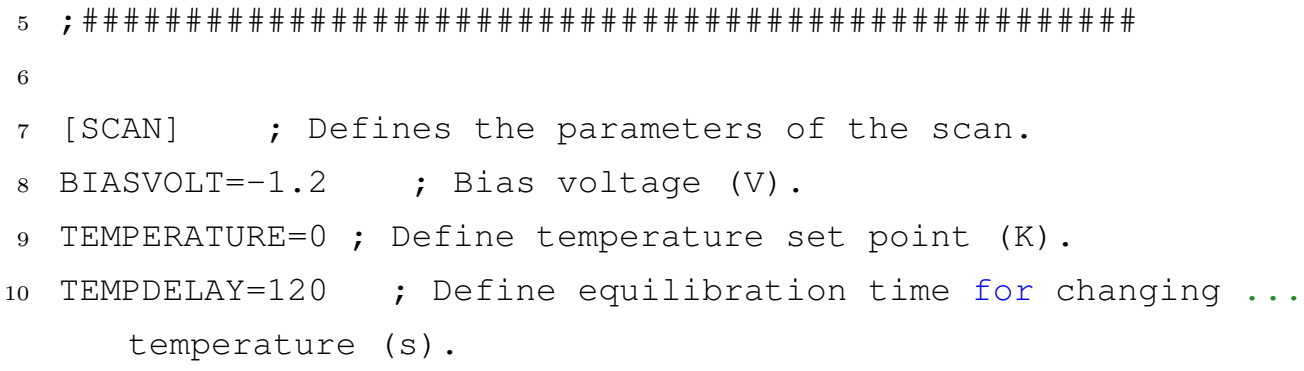




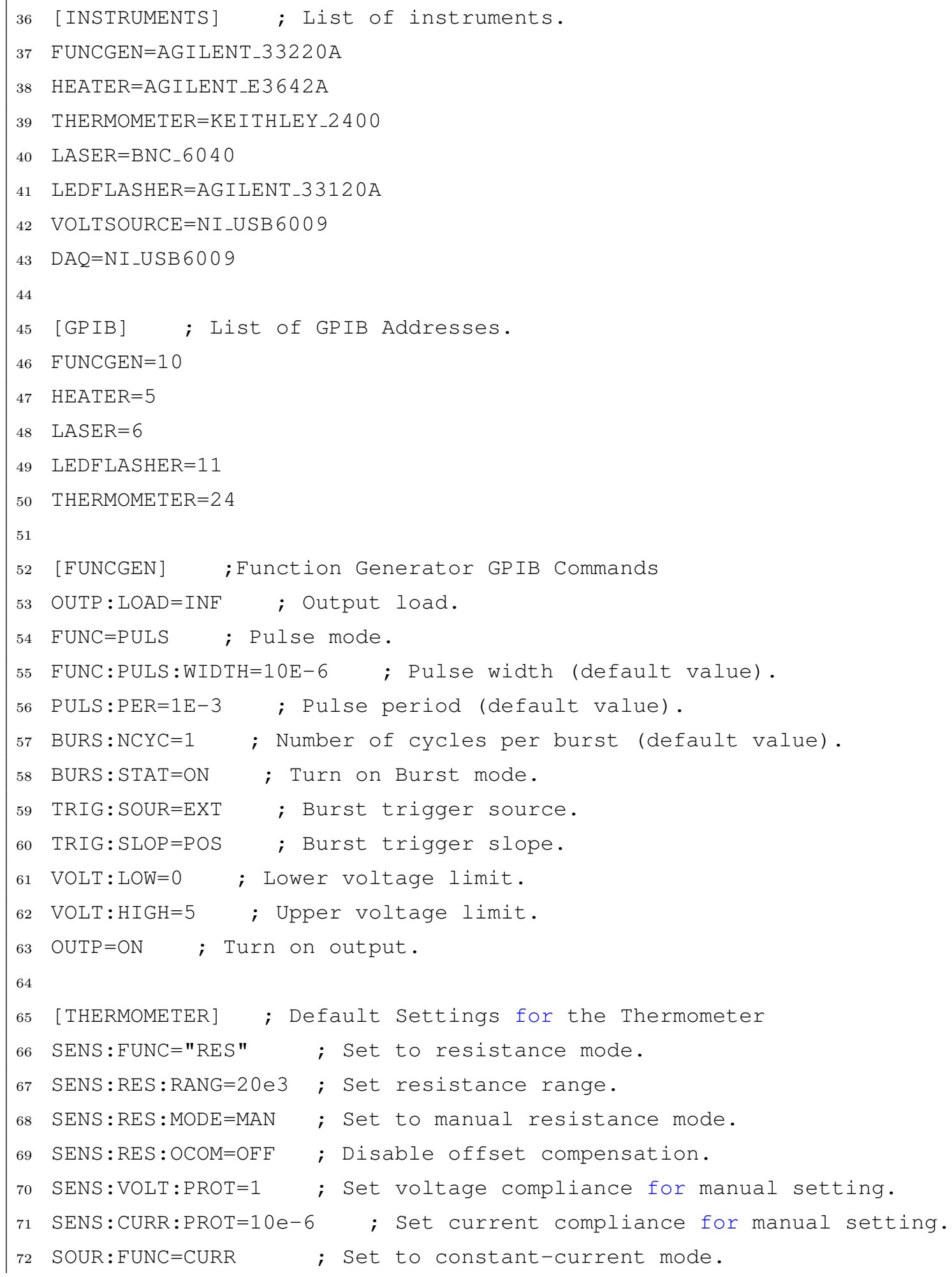




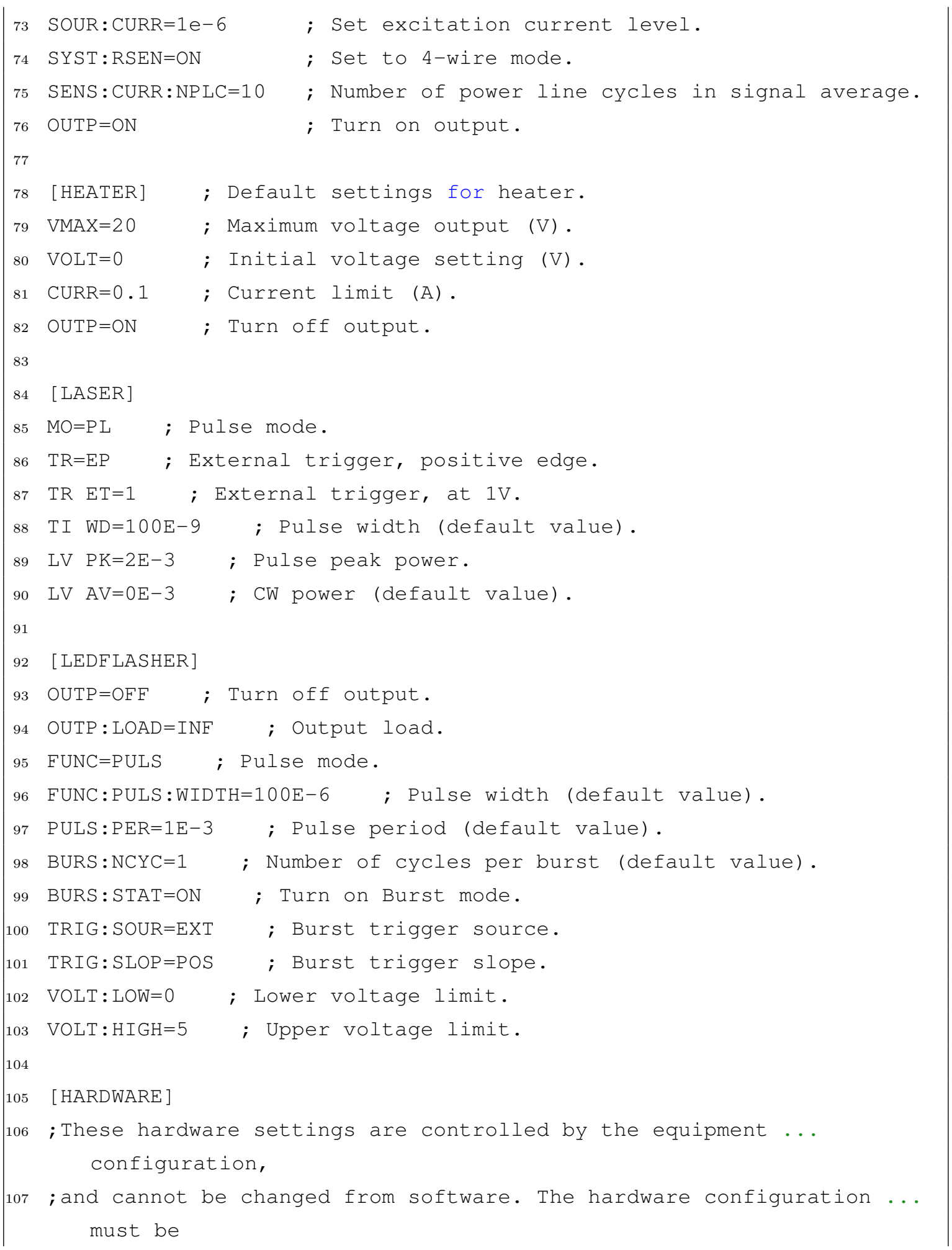




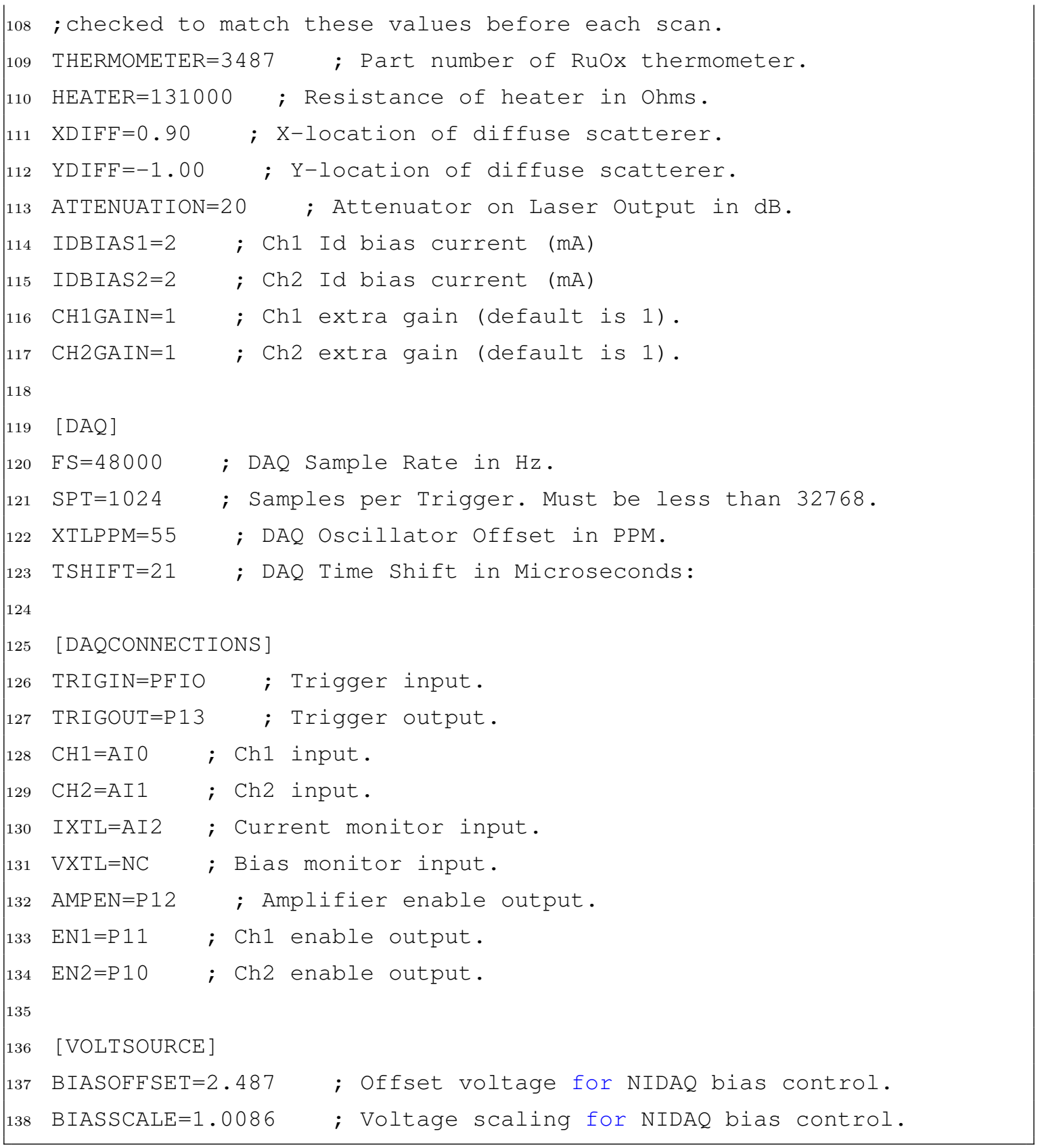

\section{ConnectAndInitializeGPIBInstruments.m:}

1 oDefine Global Variables:

2 global VFunctionGenerator VHeater VLaser VLEDFlasher VThermometer

3

4 oConnect to Function Generator: 


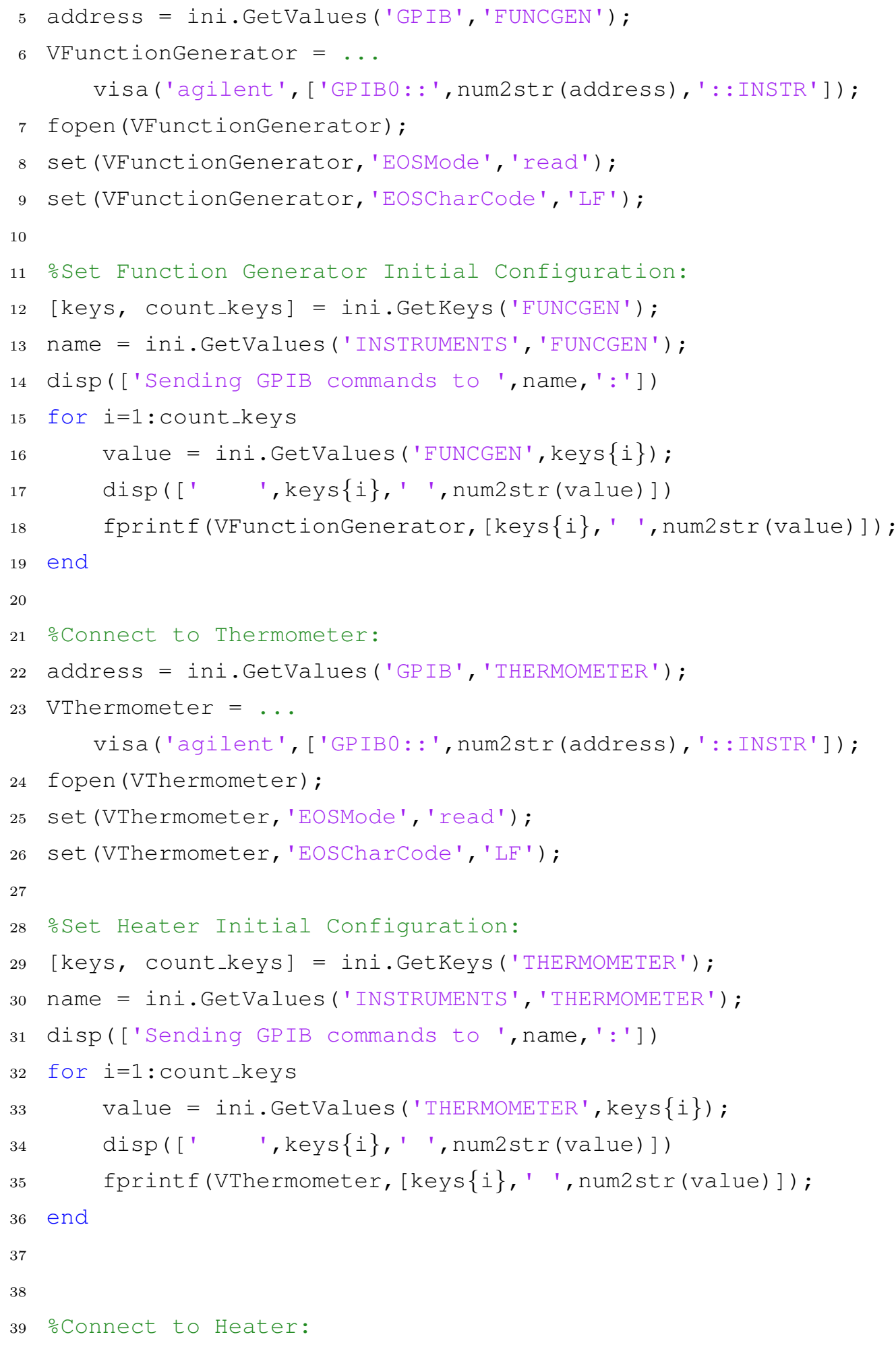




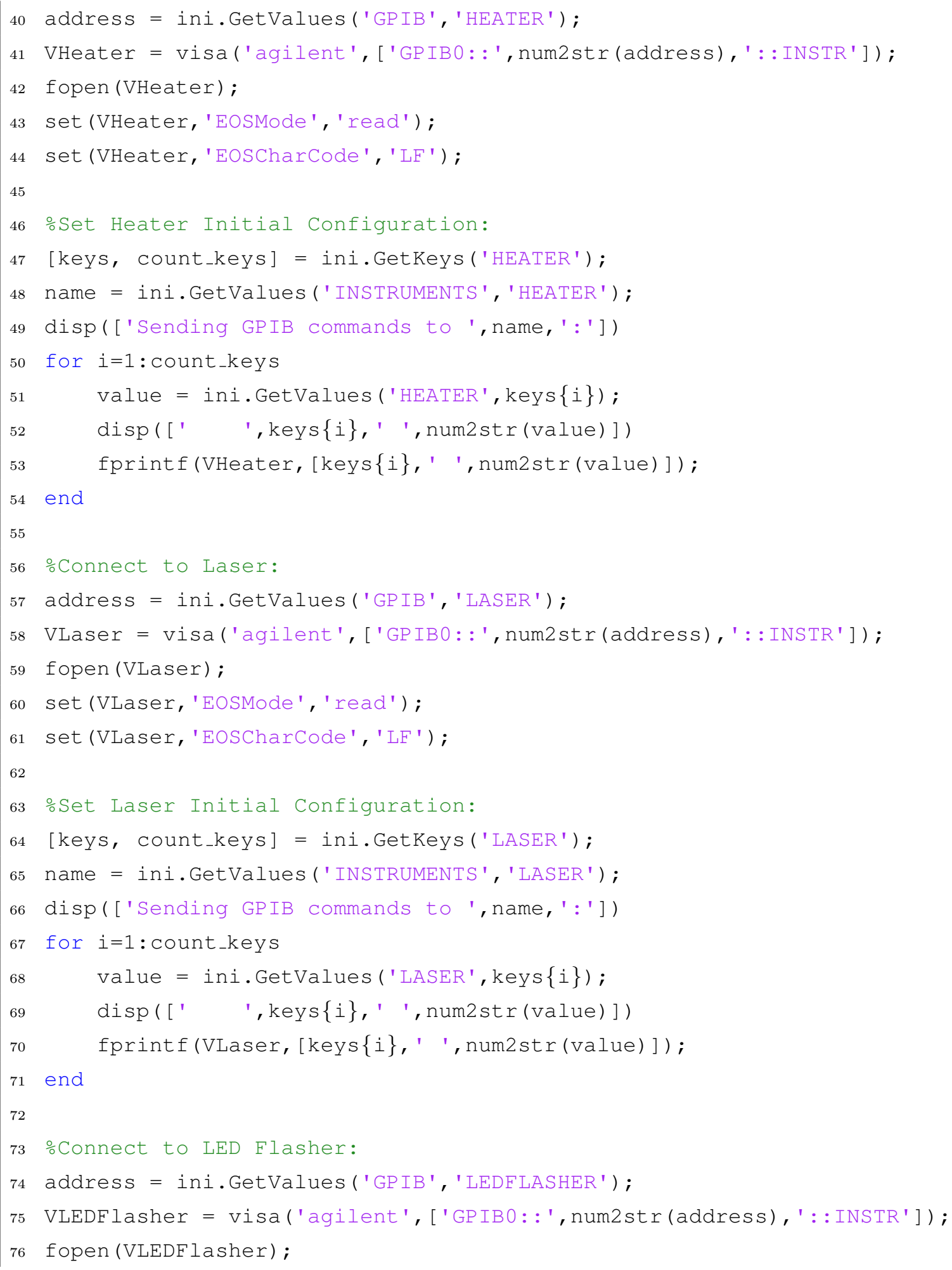




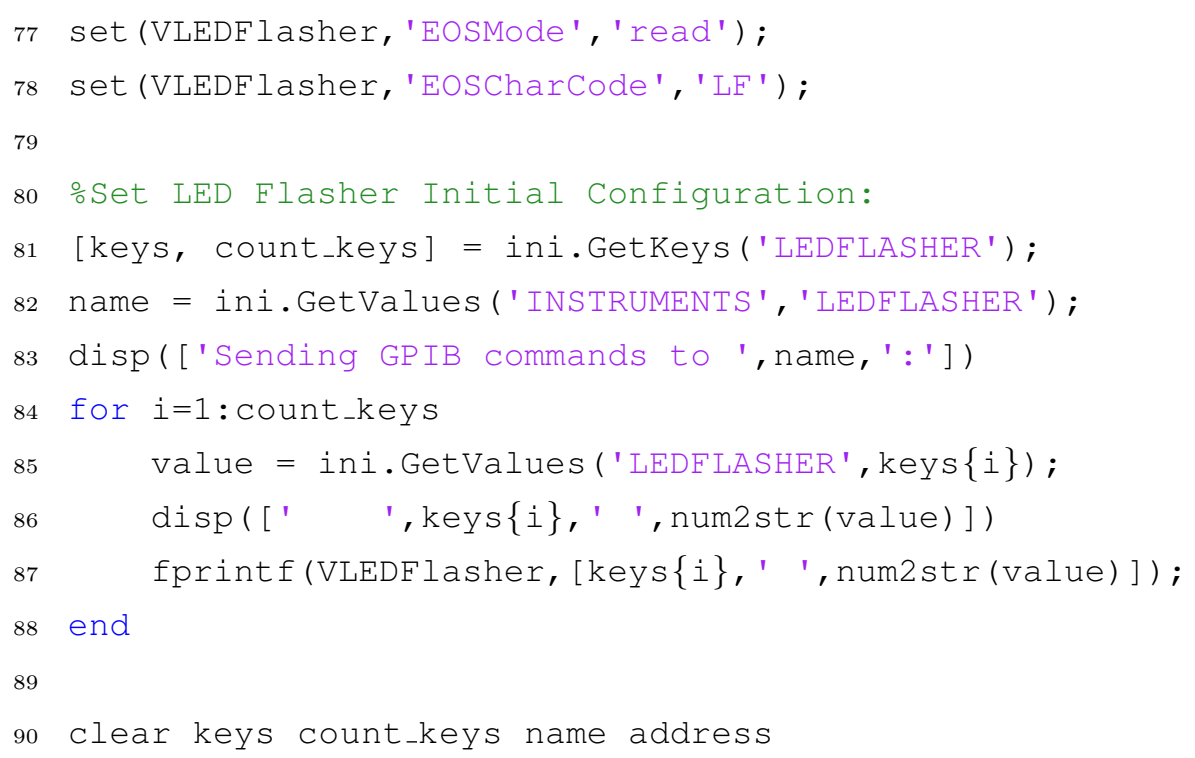

ConnectAndInitializeNIDAQ.m:

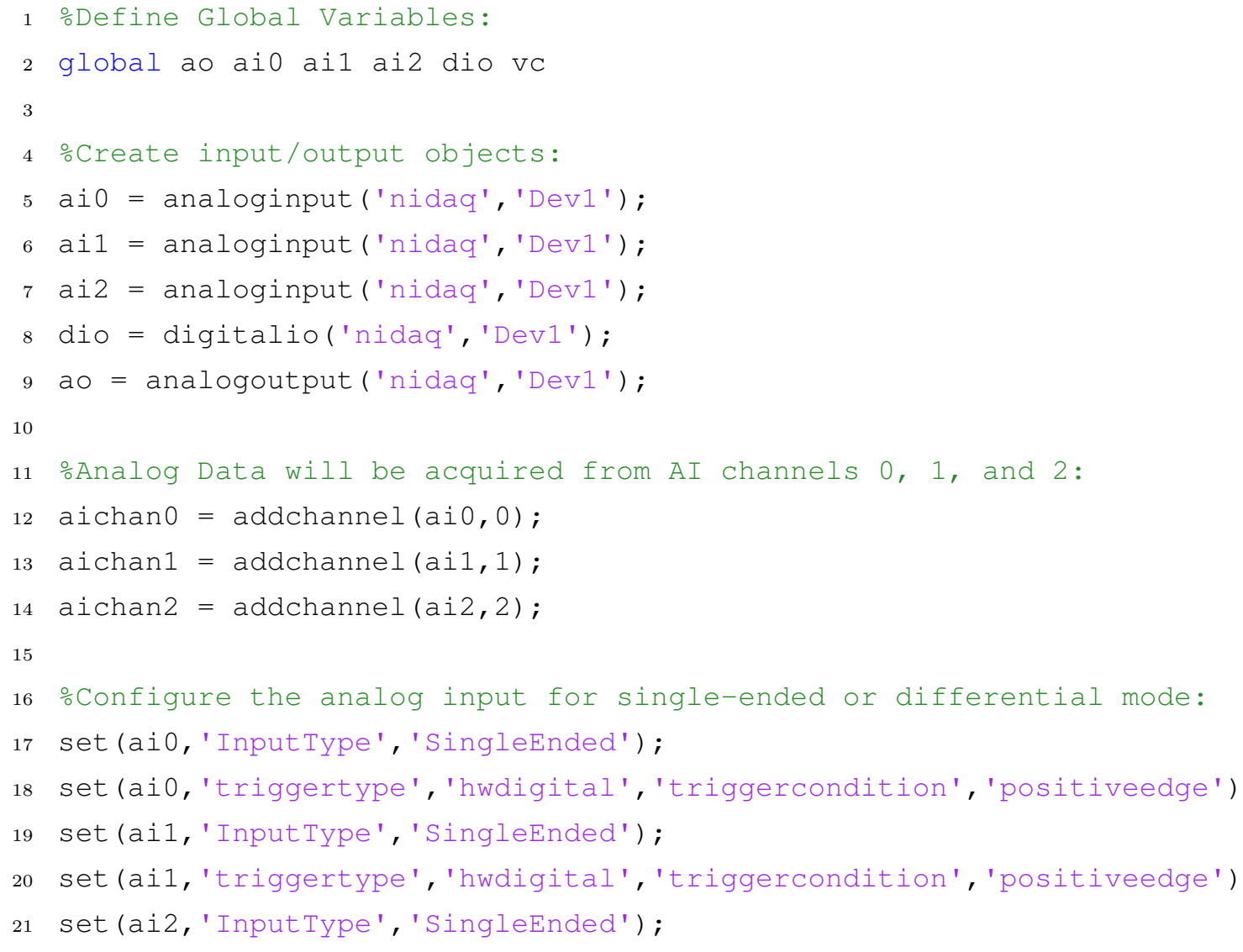




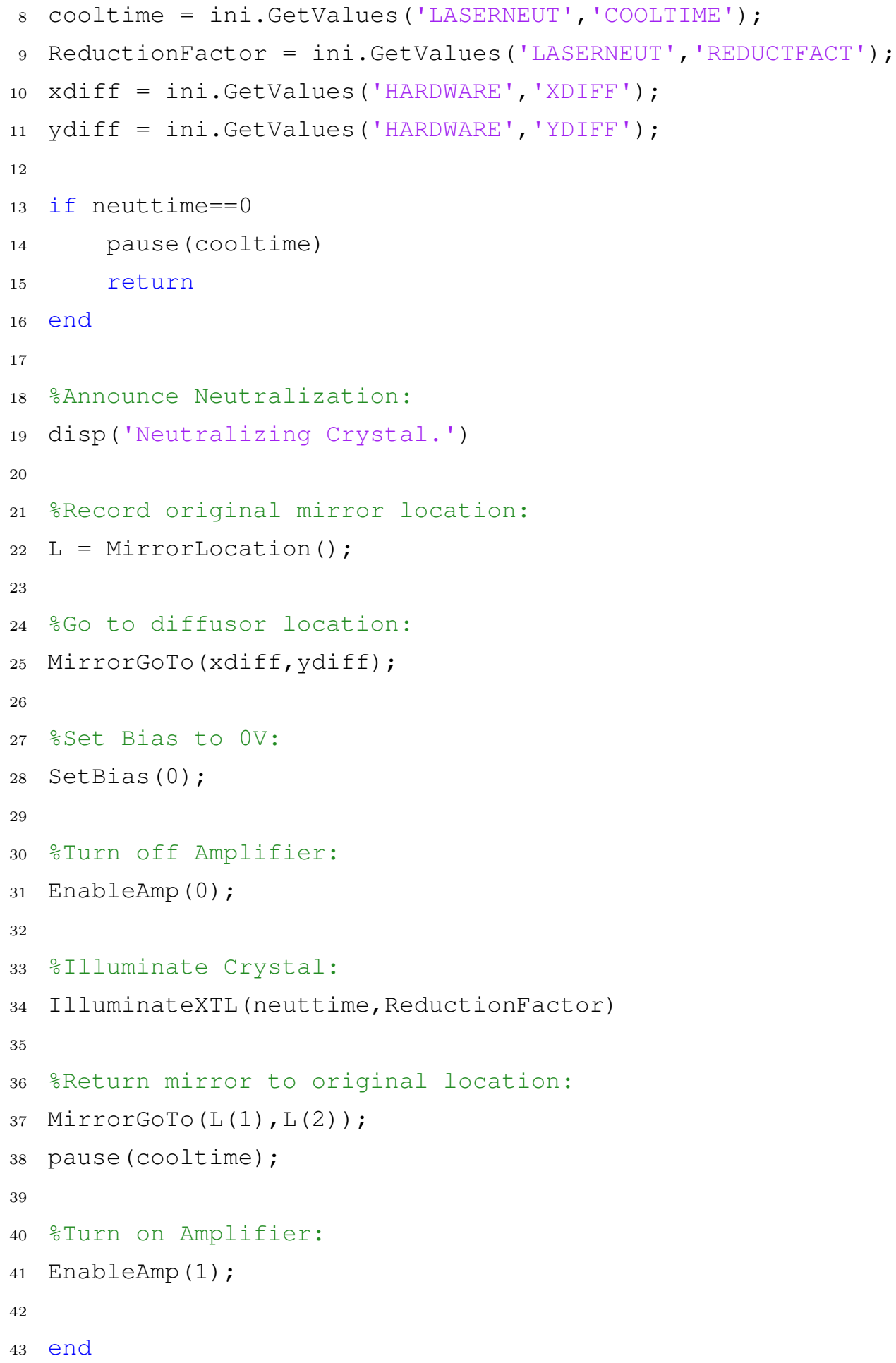




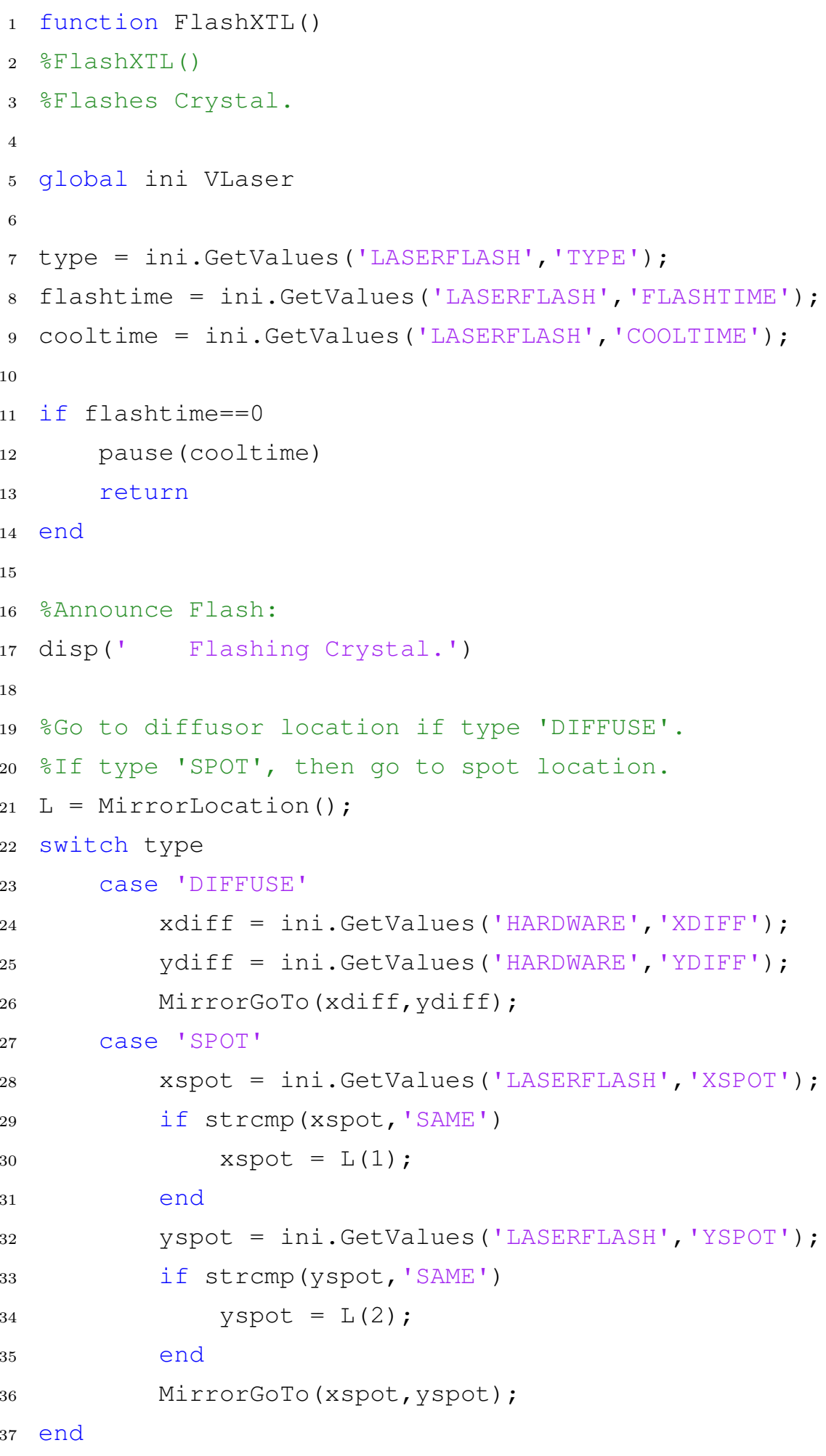




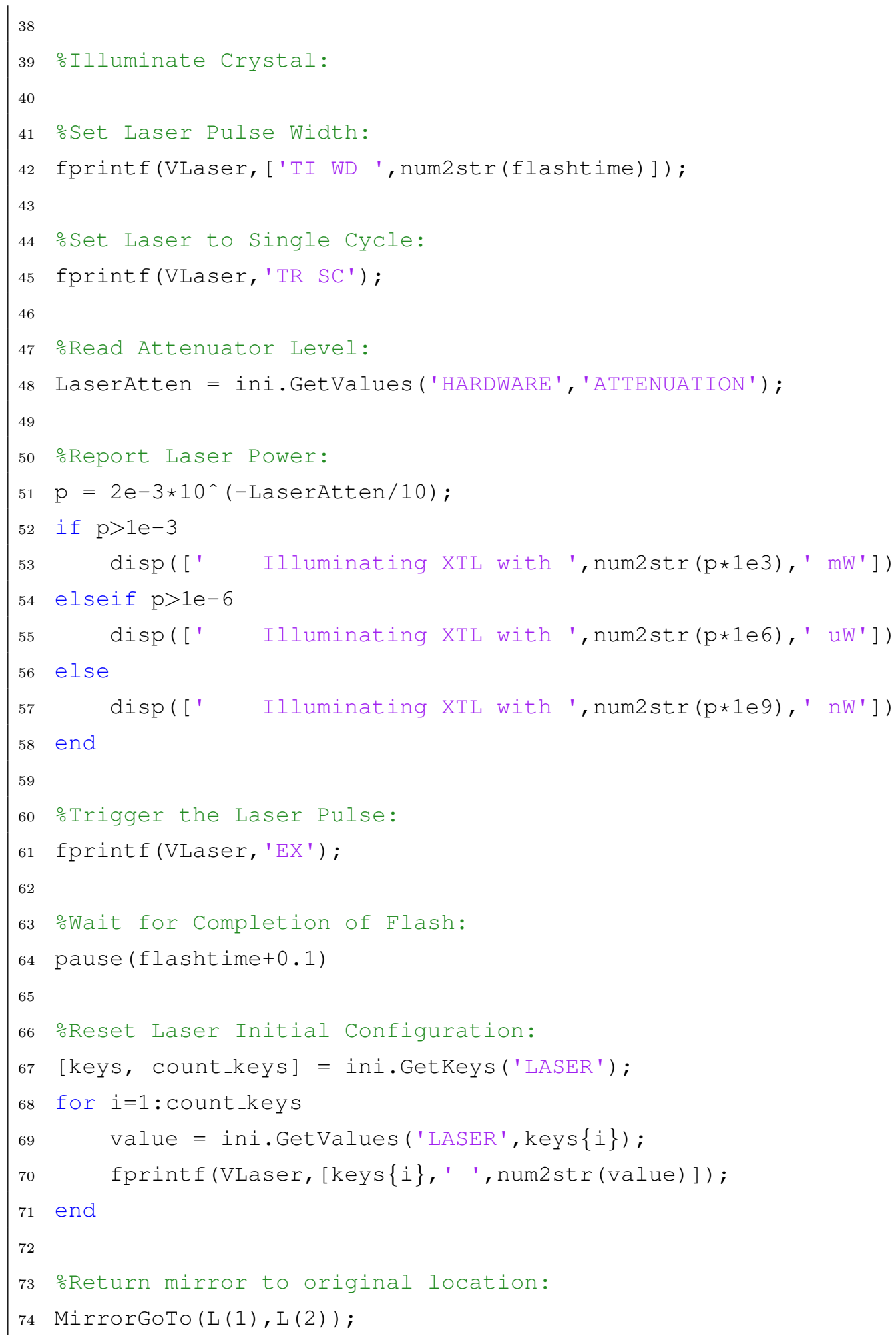




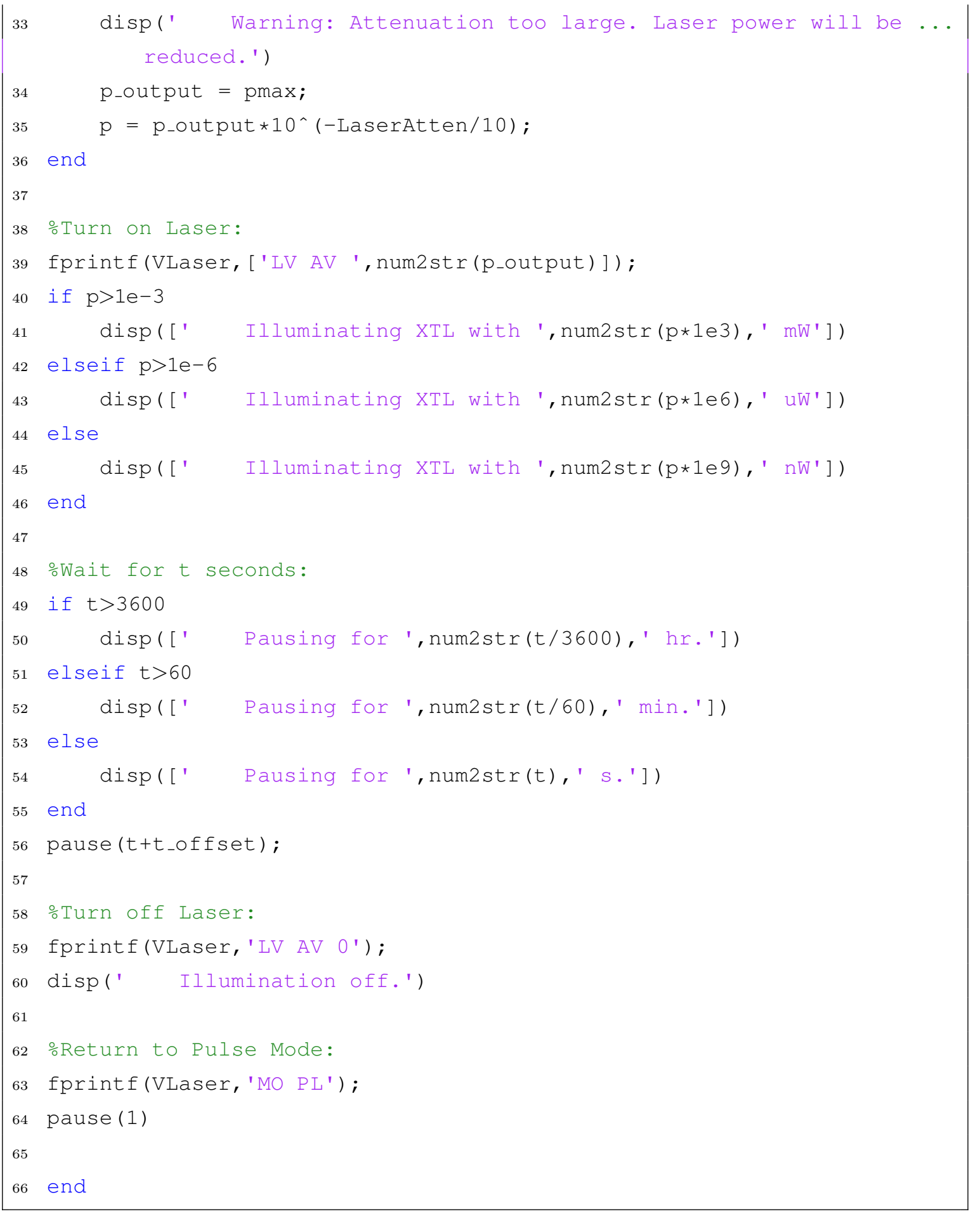




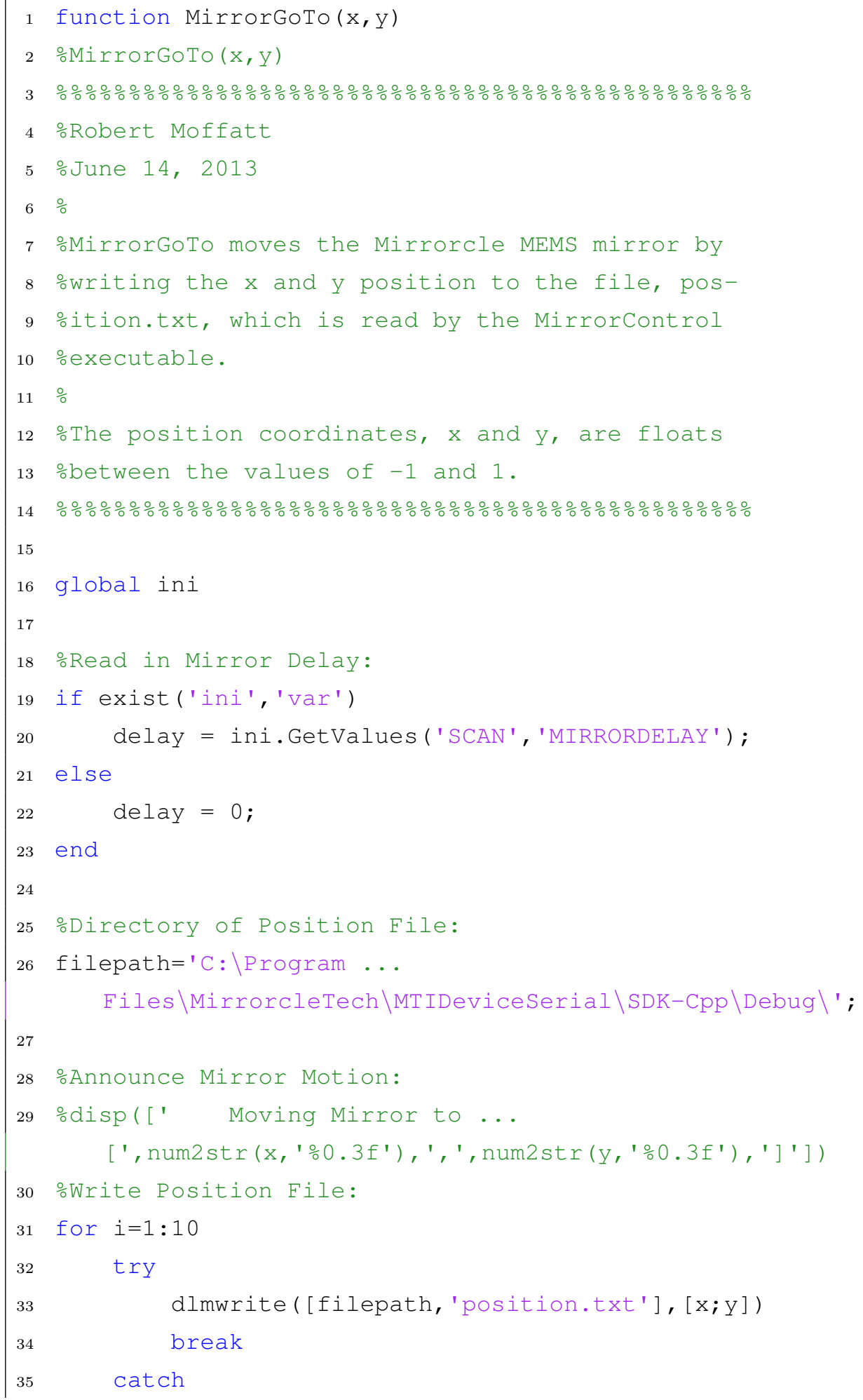




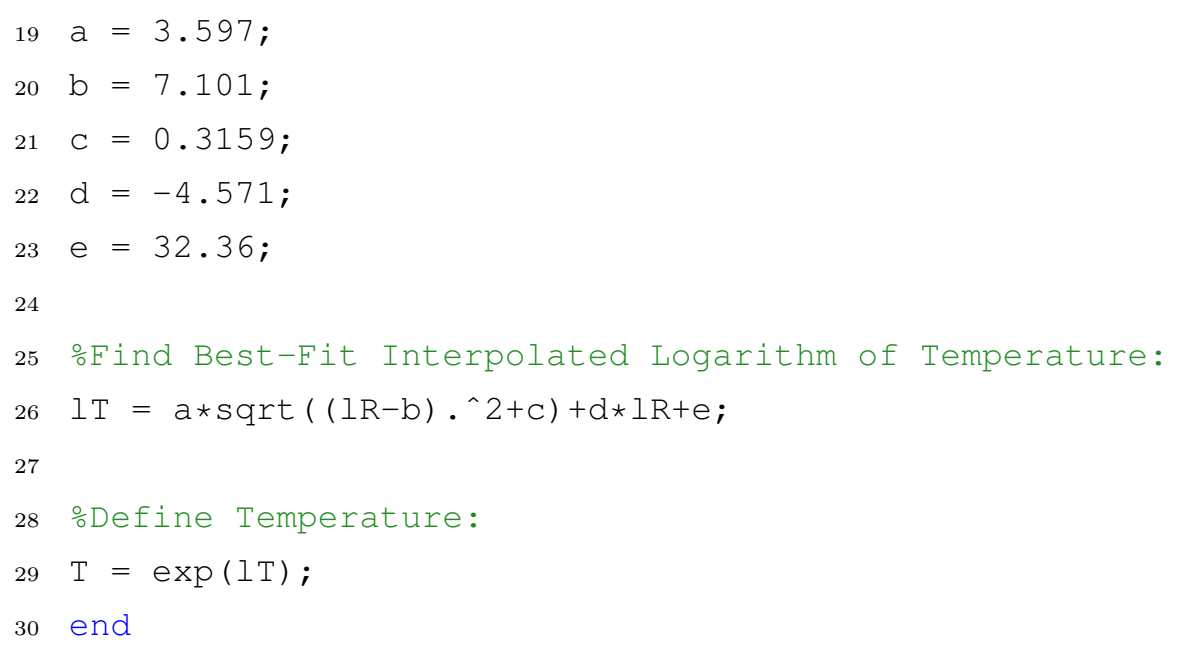

SaveVariables.m:

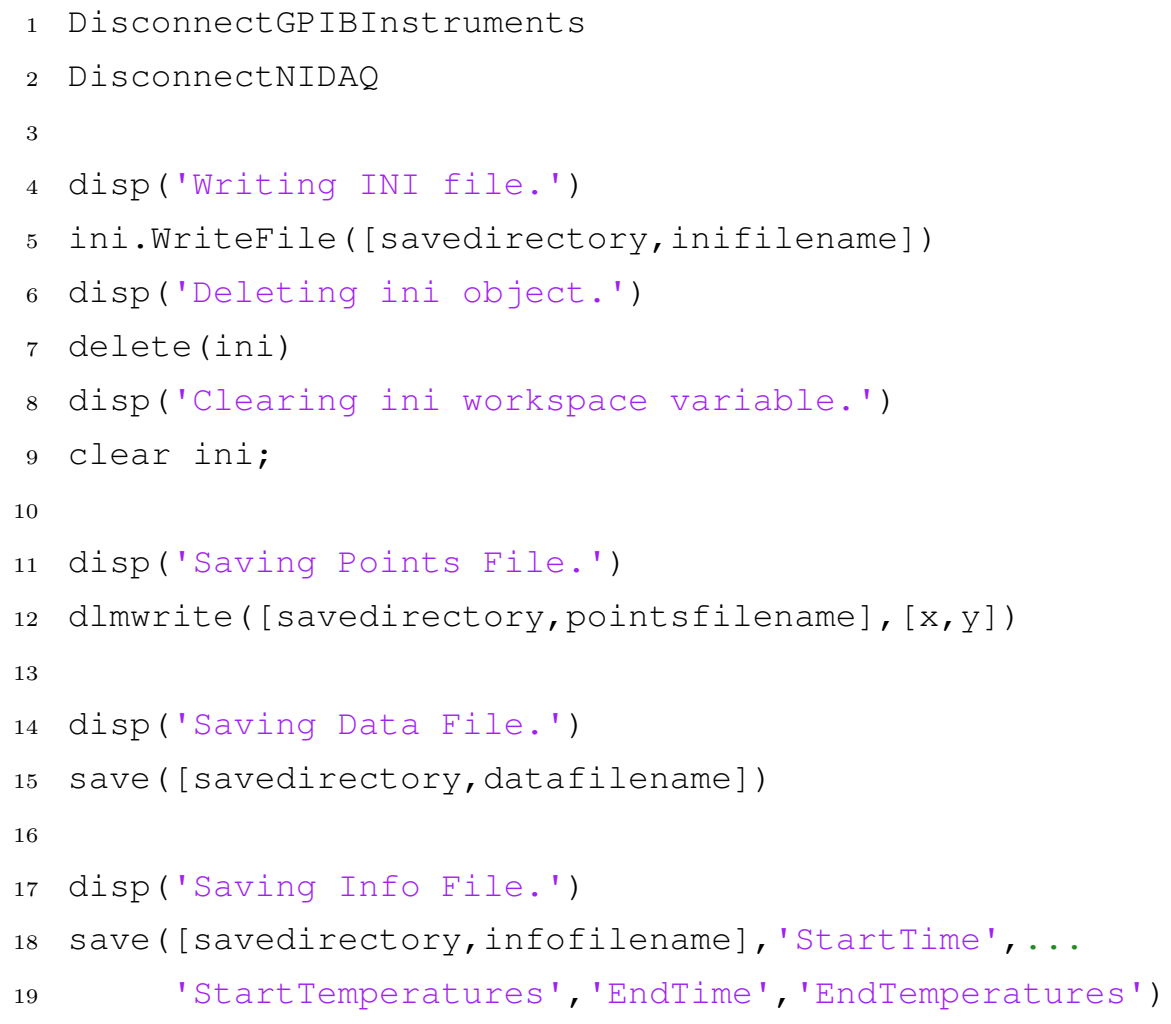

DisconnectGPIBInstruments.m: 


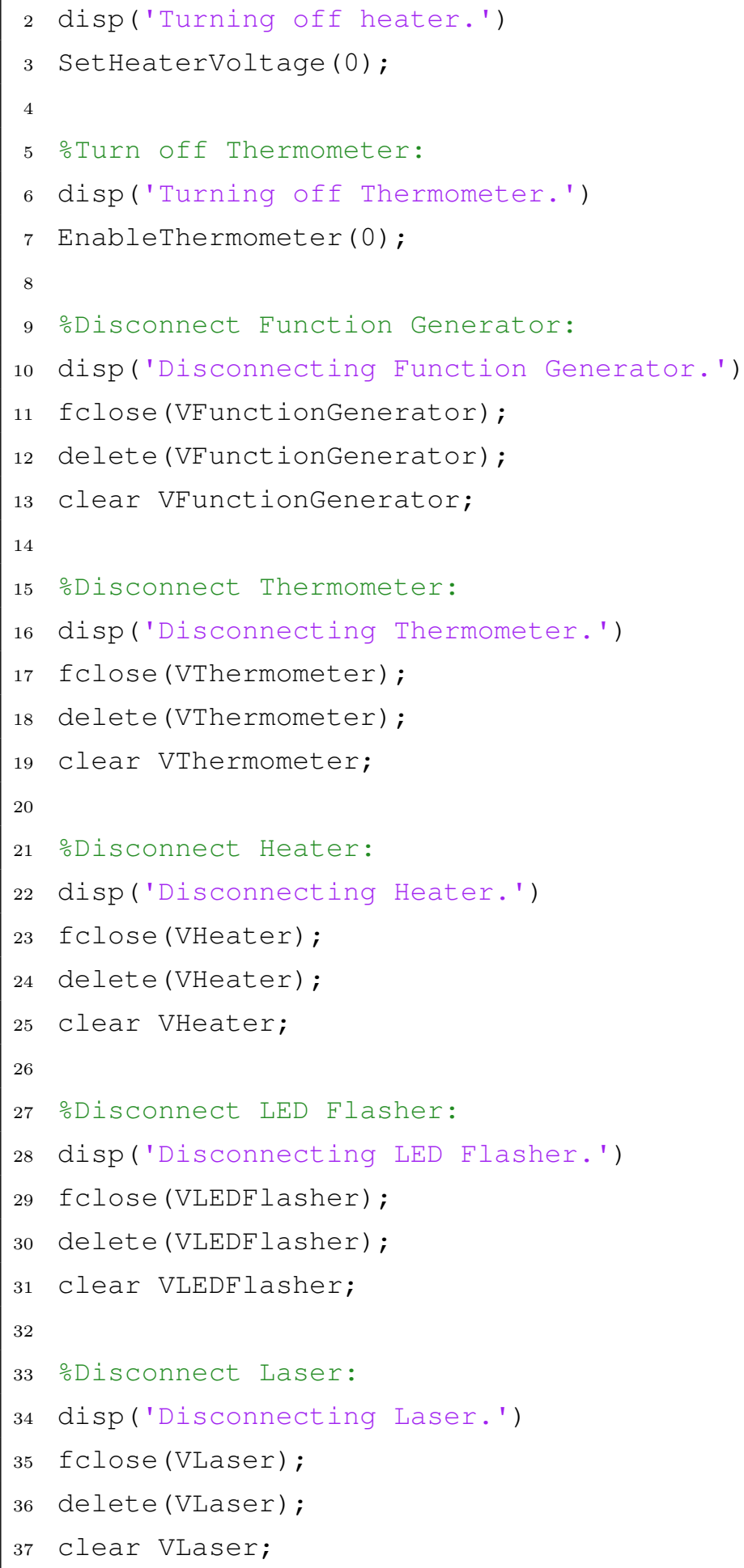




\section{DisconnectNIDAQ.m:}

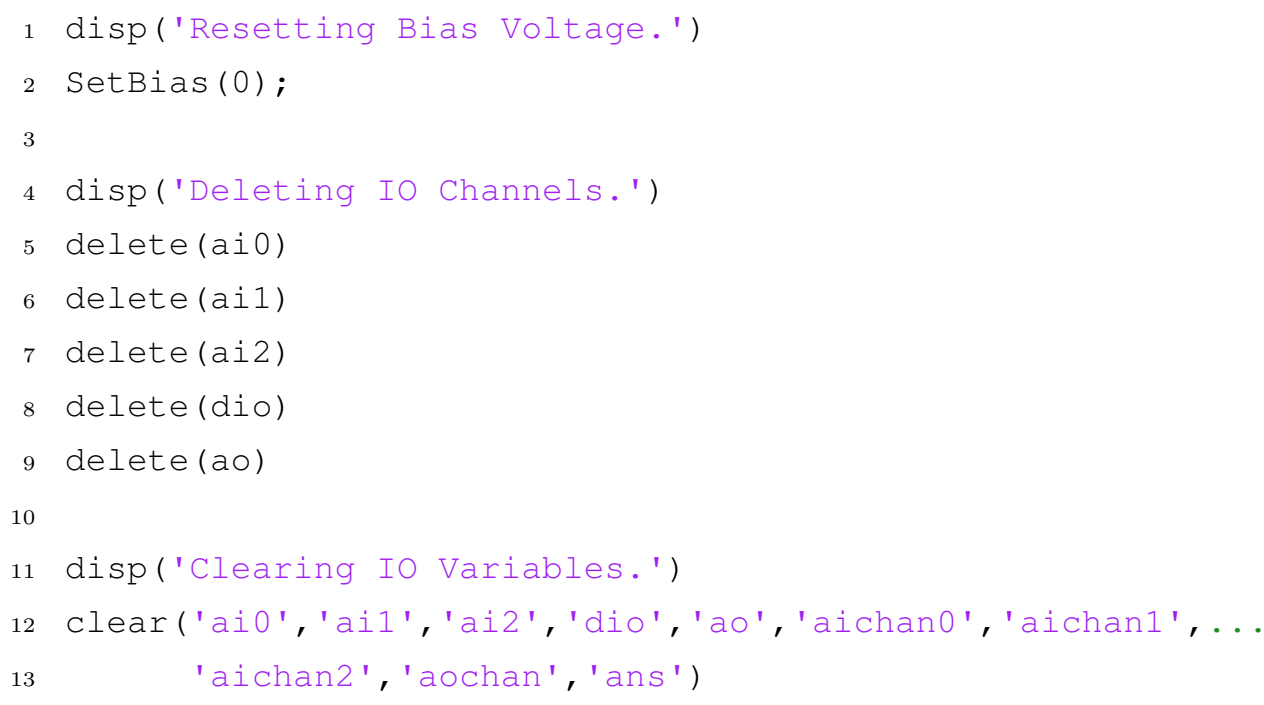

\section{RsyncScriptsToE.m:}

1 system(['rsync --verbose --progress --stats --compress ',..

2 ' --recursive --times --links --chmod=ugo+rwx ',...

3 ' .. / / cygdrive/e/CDMS/MATLAB/', RunName, ' / ' ] )

\section{RsyncDataToNero.m:}

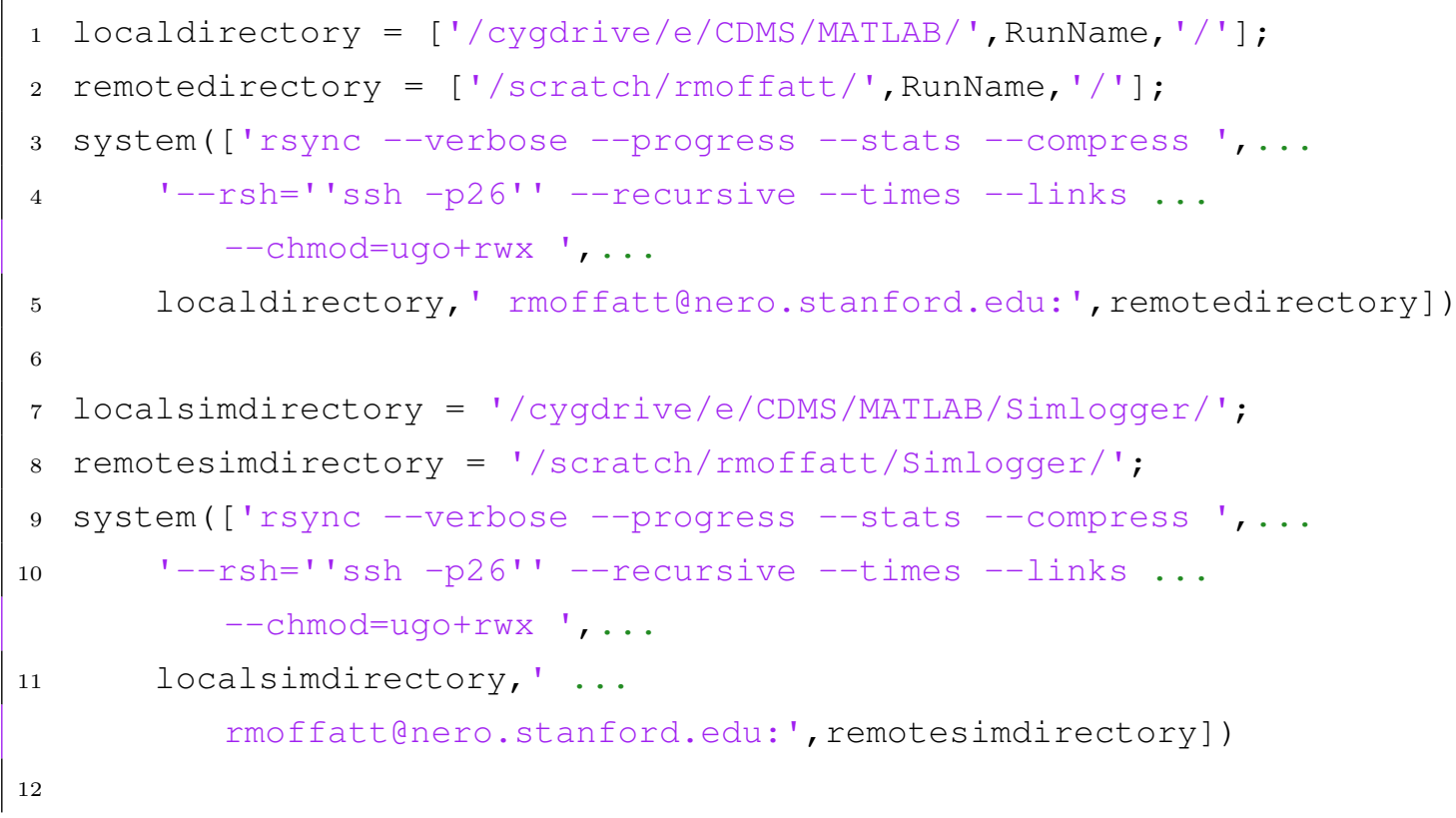




\section{Bibliography}

[1] C. L. Bennett et al., Nine-year wilkinson microwave anisotropy probe (wmap) observations: Cosmological parameter results. The Astrophysical Journal Supplement 208, 19 October 2013.

[2] Planck Collaboration et al., Planck 2013 results. i. overview of products and scientific results. Astronomy \& Astrophysics 571, A1 November 2014.

[3] Planck Collaboration et al., Planck 2015 results. xiii. cosmological parameters. ArXiv e-prints February 2015.

[4] K. Sundqvist, Carrier transport and related effects in detectors of the cryogenic dark matter. Ph.D. thesis, University of California, Berkeley, 2012.

[5] F. Zwicky, Die rotverschiebung von extragalaktischen nebeln. Helvetica Physica Acta 6, 110-127 1933.

[6] F. Zwicky, On the masses of nebulae and of clusters of nebulae. The Astrophysical Journal 86, 217 October 1937.

[7] V. C. Rubin, N. Thonnard, and W. K. Ford, Jr., Extended rotation curves of high-luminosity spiral galaxies. iv - systematic dynamical properties, sa through sc. The Astrophysical Journal 225, L107-L111 1978.

[8] V. C. Rubin, W. K. J. Ford, and N. . Thonnard, Rotational properties of 21 sc galaxies with a large range of luminosities and radii, from ngc 4605 $/ \mathrm{r}=4 \mathrm{kpc} /$ to ugc $2885 / \mathrm{r}=122 \mathrm{kpc} /$. The Astrophysical Journal 238, 471-487 June 1980. 
[9] E. Corbelli and P. Salucci, The extended rotation curve and the dark matter halo of m33. Monthly Notices of the Royal Astronomical Society $\mathbf{3 1 1}$, 441-447 2000.

[10] S. Deluca, Extended rotation curve of M33., https ://commons . wikimedia. org/wiki/File:M33_rotation_curve_HI.gif, 2014, [Online; accessed Nov. 2015].

[11] R. J. Nemiroff, The Matter of the Bullet Cluster., http://apod.nasa.gov/ apod/ap080823.html, 2008, [Online; accessed Nov. 2015].

[12] User:Cosmo0, Dark matter halo., https://commons.wikimedia.org/wiki/ File:Dark_matter_halo.png, 2008, [Online; accessed Nov. 2015].

[13] M. Milgrom, A modification of the newtonian dynamics as a possible alternative to the hidden mass hypothesis. The Astrophysical Journal 270, 365-370 1983.

[14] J. D. Bekenstein, Relativistic gravitation theory for the modified newtonian dynamics paradigm. Phys. Rev. D 70, 083509 Oct 2004.

[15] P. D. Mannheim and J. G. O'Brien, Galactic rotation curves in conformal gravity. Journal of Physics Conference Series 437, 012002 April 2013.

[16] D. Clowe et al., A direct empirical proof of the existence of dark matter. The Astrophysical Journal 648, L109-L113 2006.

[17] G. Hinshaw et al., Three-year wilkinson microwave anisotropy probe (wmap) observations: Temperature analysis. The Astrophysical Journal Supplement Series 170, 288-334 June 2007.

[18] ESA and the Planck Collaboration, Planck cosmic recipe., http://www.esa. int/spaceinimages/Images/2013/03/Planck_cosmic_recipe, 2013, [Online; accessed Nov. 2015]. 
[19] J. L. Feng, Dark matter candidates from particle physics and methods of detection. Annual Review of Astronomy and Astrophysics 48, 495-545 September 2010.

[20] S. P. Martin, a Supersymmetry Primer. Perspectives On Supersymmetry. Series: Advanced Series on Directions in High Energy Physics 18, 1-98 July 1998.

[21] G. Jungman, M. Kamionkowski, and K. Griest, Supersymmetric dark matter. Physics Reports 267, 195-373 March 1996.

[22] K.-Y. Choi, J. E. Kim, and L. Roszkowski, Review of axino dark matter. Journal of Korean Physical Society 63, 1685-1695 November 2013.

[23] M. Ackermann et al., Searching for dark matter annihilation from milky way dwarf spheroidal galaxies with six years of fermi large area telescope data. Physical Review Letters 115, 231301 December 2015.

[24] G. Aad et al., Search for new phenomena in events with a photon and missing transverse momentum in $\mathrm{p} p$ collisions at $\sqrt{s}=8$ tev with the atlas detector. Physical Review D 91, 012008 January 2015.

[25] CMS Collaboration, Search for new phenomena in monophoton final states in proton-proton collisions at $\sqrt{s}=8$ tev. ArXiv e-prints October 2014 .

[26] CMS Collaboration, Search for physics beyond the standard model in final states with a lepton and missing transverse energy in protonproton collisions at $\sqrt{s}=8$ tev. ArXiv e-prints August 2014 .

[27] ATLAS Collaboration, Search for new particles in events with one lepton and missing transverse momentum in $p p$ collisions at $\sqrt{s}=8$ tev with the atlas detector. ArXiv e-prints July 2014.

[28] K. Schneck, Search for Low-Mass Dark Matter with SuperCDMS Soudan and Study of Shorted Electric Field Configurations in CDMS Detectors. Ph.D. thesis, Stanford University, 2015. 
[29] A. K. Drukier, K. Freese, and D. N. Spergel, Detecting cold dark-matter candidates. Phys. Rev. D 33, 3495-3508 Jun 1986.

[30] G. R. Knapp, S. D. Tremaine, and J. E. Gunn, The global properties of the galaxy. $\mathrm{i}$ - the hi distribution outside the solar circle. Astronomical Journal 83, 1585-1593 December 1978.

[31] F. J. Kerr and D. Lynden-Bell, Review of galactic constants. Monthly Notices of the Royal Astronomical Society 221, 1023-1038 1986.

[32] J. A. R. Caldwell and I. M. Coulson, Milky way rotation and the distance to the galactic center from cepheid variables. Astronomical Journal 93, 1090-1105 May 1987.

[33] J. Lewin and P. Smith, Review of mathematics, numerical factors, and corrections for dark matter experiments based on elastic nuclear recoil. Astroparticle Physics 6, 87-1121996.

[34] R. B. Thakur and A. Anderson, R133 phonon of resolution. CDMS Internal Note July 2012, http://titus.stanford.edu/cdms_restricted/Soudan/ R133/ebook/120710_2/index.html.

[35] P. Cushman et al., Snowmass cf1 summary: Wimp dark matter direct detection. ArXiv e-prints October 2013.

[36] P. R. Kafle, S. Sharma, G. F. Lewis, and J. Bland-Hawthorn, On the shoulders of giants: Properties of the stellar halo and the milky way mass distribution. The Astrophysical Journal 794, 59 October 2014.

[37] S. Gillessen et al., Monitoring stellar orbits around the massive black hole in the galactic center. The Astrophysical Journal 692, 10752009.

[38] P. J. McMillan, Mass models of the milky way. Monthly Notices of the Royal Astronomical Society 414, 2446-2457 July 2011. 
[39] B. Cornell, SuperCDMS Soudan: High Threshold Analysis. CDMS Internal Note January 2016, http://cdms . berkeley. edu/wiki/lib/exe/fetch. php?media=collaboration:160108: colab_meeting_ht_2016.pdf.

[40] United Nations Scientific Committee on the Effects of Atomic Radiation, Sources and Effects of Ionizing Radiation, Annex B (United Nations, New York, 2008).

[41] P. Redl, Accurate Simulations of ${ }^{206} \mathbf{P b}$ Recoils in SuperCDMS. Journal of Low Temperature Physics 176, 937-942 2014.

[42] J. Yen, Phonon Sensor Dynamics For Cryogenic Dark Matter Search Experiment: A Study Of Quasiparticle Transport In Aluminum Coupled To Tungsten Transition Edge Sensors. Ph.D. thesis, Stanford University, 2015.

[43] B. Shank, Testing and characterization of supercdms dark matter detectors. Ph.D. thesis, Stanford University, 2014.

[44] B. Cabrera et al., Oblique propagation of electrons in [100] ge crystals. CDMS Internal Note September 2009.

[45] E. Haller, G. Hubbard, W. Hansen, and A. Seeger, Divacancy-hydrogen complexes in dislocation-free high-purity germanium. Proceedings of the International Conference on Radiation Effects in Semiconductors Sep 1976.

[46] M. Pyle, B. Serfass, and K. Sundquist, Qi/qo studies on the izip. CDMS Internal Note March 2009, http://titus.stanford.edu/cdms_restricted/ mpyle1/ebooks/IZIP2/qiqo_issues/IZIP2_qiqo.htm1.

[47] R. Mahapatra, J. Sander, R. Bunker, and D. Nelson, Apparent incorrect yield qshared events or why there is a funnel. CDMS Internal Note May 2010, http://titus.stanford.edu/cdms_restricted/Soudan/ R119/ebook/041209/index.html.

[48] N. W. Ashcroft and N. D. Mermin, Solid State Physics (Brooks/Cole Cengage Learning, Belmont, CA, 1976). 
[49] C. Kittel, Introduction to Solid State Physics (John Wiley \& Sons, Inc, Hoboken, NJ, 2005).

[50] GlobalSino, Band Structure of Germanium., http://www.globalsino. com/micro/1/1micro9970.html, 2012, [Online; accessed Nov. 2015].

[51] J. Singleton, Band theory and electronic properties of solids (Oxford University Press, Oxford, 2001).

[52] F. Bloch, Reminiscences of Heisenberg and the early days of quantum mechanics. Physics Today 23-27 December 1976.

[53] J. M. Ziman, Electrons and phonons: the theory of transport phenomena in solids (Oxford University Press, Oxford, 2001).

[54] C. Herring and E. Vogt, Phys. Rev. 101, 9941956.

[55] K. M. Sundqvist et al., A measurement of electron and hole drift velocities in a germanium $\langle 100\rangle$; cdms detector, at a temperature of 31 millikelvin. AIP Conference Proceedings 1185, 128-131 2009.

[56] C. Jacoboni and L. Reggiani, Rev. Mod. Phys. 55, 6451983.

[57] A. Lee, Rev. Sci. Instr. 60, 3315-3322 1989.

[58] R. Moffatt et al., Spatial imaging of charge transport in germanium at low temperature. Journal of Low Temperature Physics 176, 943-951 2014.

[59] Mirrorcle Technologies, Inc., Gimbal-less Dual-Axis MEMS mirror Type A1S1 (1.7mm diameter),, http://mirrorcletech.com/.

[60] A. Lee, Cryogenic phonon-mediated particle detectors for dark matter searches and neutrino physics. Ph.D. thesis, Stanford University, 1993.

[61] R. Bhatia et al., A three-stage helium sorption refrigerator for cooling of infrared detectors to $\mathbf{2 8 0} \mathbf{m k}$. Cryogenics 40, 685 - 6912000. 
[62] G. Boisset, Luxpop: Index of Refraction, Thin film, Optical simulation and ray tracing., http://www. luxpop.com/, [Online; accessed Nov. 2015].

[63] S. W. Leman, Invited Review Article: Physics and Monte Carlo techniques as relevant to cryogenic, phonon, and ionization readout of Cryogenic Dark Matter Search radiation detectors. Review of Scientific Instruments 83, 091101 September 2012.

[64] A. Broniatowski, Intervalley scattering of hot electrons in germanium at millikelvin temperatures. Journal of Low Temperature Physics 176, 860-869 2014.

[65] M.-C. Piro et al., Hot carrier trapping in high-purity and doped germanium crystals at millikelvin temperatures. Journal of Low Temperature Physics 176, 796-801 2014.

[66] A. Phipps, K. Sundqvist, A. Lam, and B. Sadoulet, Optically induced measurement of electron and hole drift velocities in a germanium $\langle\mathbf{1 0 0}\rangle$ cdms detector at 50 mk. Journal of Low Temperature Physics 167, 111211182012 .

[67] K. Sundqvist, A. Phipps, A. Dixit, and B. Sadoulet, Transport and twospecies capture of electrons and holes in ultrapure germanium at millikelvin temperature. Journal of Low Temperature Physics 176, 188-193 2014.

[68] G. Shaviv, The Life of Stars (Springer Publishing, Berlin, 2009), p. 38.

[69] S. Chadrasekhar, An Introduction to the Study of Stellar Structure (Dover Publications, Inc., New York, 1939).

[70] E. W. Weisstein, Lane-Emden Differential Equation. From MathWorld-A Wolfram Web Resource. 2015.

[71] Y. Sofue et al., Astrophysical Journal 523, 136-146 1999. 
[72] M. E. Peskin and D. V. Schroeder, An Introduction to Quantum Field Theory (Westview Press, Boulder, Colorado, 1995).

[73] P. W. Graham, D. E. Kaplan, S. Rajendran, and M. T. Walters, Semiconductor probes of light dark matter. Physics of the Dark Universe 1, 32-49 November 2012.

[74] R. Essig et al., Direct Detection of sub-GeV Dark Matter with Semiconductor Targets. ArXiv e-prints September 2015.

[75] J. R. King, Approximate solutions to a nonlinear diffusion equation. Journal of Engineering Mathematics 22, 53-72 .

[76] J. Jochum et al., Dynamics of radiation induced quasiparticles in superconducting tunnel junction detectors. Annalen der Physik 505, 611-634 1993.

[77] M. Pyle, Optimizing the Design and Analysis of Cryogenic Semiconductor Dark Matter Detectors for Maximum Sensitivity. Ph.D. thesis, Stanford University, 2012.

[78] C. Doran and A. Lasenby, Geometric Algebra for Physicists (Cambridge University Press, Cambridge, 2005).

[79] G. Kälbermann, Ehrenfest theorem, galilean invariance and nonlinear schrödinger equations. ArXiv e-prints 2003.

[80] B. K. Ridley, Quantum processes in semiconductors (Clarendon Press, Oxford, 2005).

[81] H. Yokoyama and K. Ujihara, Spontaneous emission and laser oscillation in microcavities (CRC Press, Boca Raton, FL, 1995). 